Supporting Information

\title{
Substituent Effects and the Energetics of Non-Catalyzed Aryl Halide Aminations: A Theoretical Investigation
}

\author{
Supreeth Prasad, ${ }^{a}$ Dylan D. Rodene, ${ }^{\mathrm{b}}$ Michael B. Burkholder, ${ }^{\mathrm{b}}$ Kelling J. Donald, ${ }^{\mathrm{c*}}$ B. Frank \\ Gupton $^{\mathrm{b} *}$ \\ ${ }^{a}$ Department of Chemistry, University of California - Davis, Davis, CA 95616, United States. \\ ${ }^{\mathrm{b}}$ Department of Chemical and Life Science Engineering, Virginia Commonwealth University, Richmond, VA 23284, United States. \\ ${ }^{\mathrm{c}}$ Department of Chemistry, University of Richmond, Richmond, VA 23173, United States.
}


Table of Contents

\begin{tabular}{|c|c|}
\hline Caption Summaries & Page \\
\hline $\begin{array}{l}\text { Figure S1. Zero-point corrected and free energy barrier heights using the different } \\
\text { functionals for the non-catalyzed } 4-\mathrm{RPhX}+\mathrm{NH}_{2}{ }^{\mathrm{n}} \mathrm{Pr} \text { amination, where } \mathrm{R}=-\mathrm{H},-\mathrm{Me},- \\
\mathrm{OMe},-\mathrm{F},-\mathrm{CF}_{3},-\mathrm{COOH} \text {; and } \mathrm{X}=-\mathrm{Cl},-\mathrm{Br} \text {, and }-\mathrm{I} \text {. }\end{array}$ & $\mathrm{S} 4$ \\
\hline $\begin{array}{l}\text { Table S1. Zero-point corrected energy }\left(E_{a}^{Z P E}\right) \text { and free energy }\left(\Delta G_{a}^{298.15 K}\right) \text { changes in } \mathrm{kJ} \\
\mathrm{mol}^{-1} \text { for the } n-\mathrm{RPhX}+\mathrm{NH}_{2}{ }^{\mathrm{n}} \mathrm{Pr} \text { amination for } \mathrm{X}=\mathrm{Cl} \text {, where } \mathrm{n}=2 \text { (ortho), } 3 \text { (meta) or } 4 \\
\text { (para) and } \mathrm{R}=-\mathrm{H},-\mathrm{Me},-\mathrm{OMe},-\mathrm{F},-\mathrm{CF}_{3},-\mathrm{COOH} \text {, and }-\mathrm{CN} \text {. }\end{array}$ & S5 \\
\hline $\begin{array}{l}\text { Table S2. Zero-point corrected energy }\left(E_{a}^{Z P E}\right) \text { and free energy }\left(\Delta G_{a}^{298.15 K}\right) \text { changes in } \mathrm{kJ} \\
\mathrm{mol}^{-1} \text { for the } n-\mathrm{RPhX}+\mathrm{NH}_{2}{ }^{\mathrm{n}} \mathrm{Pr} \text { amination for } \mathrm{X}=\mathrm{Br} \text {, where } \mathrm{n}=2 \text { (ortho), } 3 \text { (meta) or } 4 \\
\text { (para) and } \mathrm{R}=-\mathrm{H},-\mathrm{Me},-\mathrm{OMe},-\mathrm{F},-\mathrm{CF}_{3},-\mathrm{COOH} \text {, and }-\mathrm{CN} \text {. }\end{array}$ & S6 \\
\hline $\begin{array}{l}\text { Table S3. Zero-point corrected energy }\left(E_{a}^{Z P E}\right) \text { and free energy }\left(\Delta G_{a}^{298.15 K}\right) \text { changes in } \mathrm{kJ} \\
\mathrm{mol}^{-1} \text { for the } n-\mathrm{RPhX}+\mathrm{NH}_{2}{ }^{\mathrm{n} P r} \text { amination for } \mathrm{X}=\mathrm{I} \text {, where } \mathrm{n}=2 \text { (ortho), } 3 \text { (meta) or } 4 \\
\text { (para) and } \mathrm{R}=-\mathrm{H},-\mathrm{Me},-\mathrm{OMe},-\mathrm{F},-\mathrm{CF}_{3},-\mathrm{COOH} \text {, and }-\mathrm{CN} \text {. }\end{array}$ & S7 \\
\hline $\begin{array}{l}\text { Table S4. Barrier heights }\left(E_{a}^{Z P E} \text { and } \Delta G_{a}^{298.15 K}\right) \text { for the amination reactions calculated at } \\
\text { the B3LYP-D3/cc-pVTZ level of theory }\left(n-\mathrm{RPhX}+\mathrm{NH}_{2} \mathrm{R}^{\prime} \rightarrow n-\mathrm{RPhNHR}+\mathrm{HX}\right) \text {, where } \\
n=4(\text { para }) ; \mathrm{R}=-\mathrm{CF}_{3},-\mathrm{CN},-\mathrm{COOH},-\mathrm{F},-\mathrm{Me},-\mathrm{OMe} \text {, and }-\mathrm{H} ; \mathrm{X}=-\mathrm{Cl},-\mathrm{Br} \text {, and }-\mathrm{I} \text {; } \\
\text { and } \mathrm{R}^{\prime}=-{ }^{\mathrm{n}} \mathrm{Pr} \text {. }\end{array}$ & S8 \\
\hline $\begin{array}{l}\text { Table S5. Barrier heights }\left(E_{a}^{Z P E} \text { and } \Delta G_{a}^{298.15 K}\right) \text { for the amination reactions calculated at } \\
\text { the } \omega \mathrm{B} 97 \mathrm{XD} / \mathrm{cc}-\mathrm{pVTZ} \text { level of theory }\left(n-\mathrm{RPhX}+\mathrm{NH}_{2} \mathrm{R}^{\prime} \rightarrow n-\mathrm{RPhNHR}+\mathrm{HX}\right) \text {, where } \\
n=4(\text { para }) ; \mathrm{R}=-\mathrm{CF}_{3},-\mathrm{CN},-\mathrm{COOH},-\mathrm{F},-\mathrm{Me},-\mathrm{OMe} \text {, and }-\mathrm{H} ; \mathrm{X}=-\mathrm{Cl},-\mathrm{Br} \text {, and }-\mathrm{I} \text {; } \\
\text { and } \mathrm{R}^{\prime}=-\mathrm{n} \mathrm{Pr} \text {. }\end{array}$ & S8 \\
\hline The Effect of Amine Substituents Discussion & S9 \\
\hline $\begin{array}{l}\text { Table S6. Zero-point corrected energy }\left(E_{a}^{Z P E}\right) \text { and free energy }\left(\Delta G_{a}^{298.15 K}\right) \text { changes in } \mathrm{kJ} \\
\mathrm{mol}^{-1} \text { for the } 4-\mathrm{MePhBr}+\mathrm{NH}_{2} \mathrm{R} \text { amination for } \mathrm{R}=-\mathrm{Me} \text {, }-\mathrm{Et},{ }^{-} \mathrm{Pr} \text {, and }{ }^{\mathrm{n}} \mathrm{Bu} \text {. }\end{array}$ & S9 \\
\hline $\begin{array}{l}\text { Table S7. Zero-point corrected energy }\left(E_{a}^{Z P E}\right) \text { and free energy }\left(\Delta G_{a}^{298.15 K}\right) \text { changes in } \mathrm{kJ} \\
\mathrm{mol}^{-1} \text { for the } 4-\mathrm{MePhCl}+\mathrm{NH}_{2}{ }^{\mathrm{n}} \mathrm{Pr} \text { amination at different temperatures. The default used } \\
\text { for thermochemistry analysis is } 25^{\circ} \mathrm{C} \text {. }\end{array}$ & $\mathrm{S} 10$ \\
\hline $\begin{array}{l}\text { Table S8. Zero-point corrected energy }\left(E_{a}^{Z P E}\right) \text { and free energy }\left(\Delta G_{a}^{298.15 K}\right) \text { changes in } \mathrm{kJ} \\
\mathrm{mol}^{-1} \text { for the } 4-\mathrm{MePhBr}+\mathrm{NH}_{2}{ }^{\mathrm{n}} \mathrm{Pr} \text { amination at different temperatures. The default used } \\
\text { for thermochemistry analysis is } 25^{\circ} \mathrm{C} \text {. }\end{array}$ & S10 \\
\hline
\end{tabular}




\begin{tabular}{|c|c|}
\hline $\begin{array}{l}\text { Table S9. Zero-point corrected energy }\left(E_{a}^{Z P E}\right) \text { and free energy }\left(\Delta G_{a}^{298.15 K}\right) \text { changes in } \\
\mathrm{kJ} \mathrm{mol}^{-1} \text { for the } \mathrm{PhBr}+\mathrm{NH}_{2}{ }^{\mathrm{n}} \mathrm{Pr} \text { amination at } 80^{\circ} \mathrm{C} \text { in different solvent environments. }\end{array}$ & S11 \\
\hline $\begin{array}{l}\text { Table S10. Coordinates for the optimized reactants, products and transition states for } \\
\text { the } n-\mathrm{RPhX}+\mathrm{NH}_{2}{ }^{\mathrm{n} P r} \text { amination for } \mathrm{X}=\mathrm{Cl} \text {, where } \mathrm{n}=2 \text { (ortho), } 3 \text { (meta) or } 4 \text { (para) } \\
\text { and } \mathrm{R}=-\mathrm{H},-\mathrm{Me},-\mathrm{OMe},-\mathrm{F},-\mathrm{CF}_{3},-\mathrm{COOH} \text {, and }-\mathrm{CN} \text {. }\end{array}$ & $\mathrm{S} 12$ \\
\hline $\begin{array}{l}\text { Table S11. Coordinates for the optimized reactants, products and transition states for } \\
\text { the } n-\mathrm{RPhX}+\mathrm{NH}_{2}{ }^{\mathrm{n}} \mathrm{Pr} \text { amination for } \mathrm{X}=\mathrm{Br} \text {, where } \mathrm{n}=2 \text { (ortho), } 3 \text { (meta) or } 4 \text { (para) } \\
\text { and } \mathrm{R}=-\mathrm{H},-\mathrm{Me},-\mathrm{OMe},-\mathrm{F},-\mathrm{CF}_{3},-\mathrm{COOH} \text {, and }-\mathrm{CN} \text {. }\end{array}$ & S19 \\
\hline $\begin{array}{l}\text { Table S12. Coordinates for the optimized reactants, products and transition states for } \\
\text { the } n-\mathrm{RPhX}+\mathrm{NH}_{2}{ }^{\mathrm{n}} \mathrm{Pr} \text { amination for } \mathrm{X}=\mathrm{I} \text {, where } \mathrm{n}=2 \text { (ortho), } 3 \text { (meta) or } 4 \text { (para) and } \\
\mathrm{R}=-\mathrm{H},-\mathrm{Me},-\mathrm{OMe},-\mathrm{F},-\mathrm{CF}_{3},-\mathrm{COOH} \text {, and }-\mathrm{CN} \text {. }\end{array}$ & S26 \\
\hline $\begin{array}{l}\text { Table S13. XYZ coordinates of the optimized reactants, products and transition states } \\
\text { for the } 4-\mathrm{MePhBr}+\mathrm{NH}_{2} \mathrm{R} \text { amination for } \mathrm{R}=-\mathrm{Me},-\mathrm{Et} \text {, }{ }^{\mathrm{n}} \mathrm{Pr} \text {, and }-{ }^{\mathrm{n}} \mathrm{Bu} \text {. }\end{array}$ & S33 \\
\hline $\begin{array}{l}\text { Table S14. XYZ coordinates of the optimized reactants, products and transition states } \\
\text { for the } 4-\mathrm{MePhCl}+\mathrm{NH}_{2}{ }^{\mathrm{n}} \mathrm{Pr} \text { amination at different temperatures. }\end{array}$ & $\mathrm{S} 35$ \\
\hline $\begin{array}{l}\text { Table S15. XYZ coordinates of the optimized reactants, products and transition states } \\
\text { for the } 4-\mathrm{MePhBr}+\mathrm{NH}_{2}{ }^{\mathrm{n}} \mathrm{Pr} \text { amination at different temperatures. }\end{array}$ & S36 \\
\hline 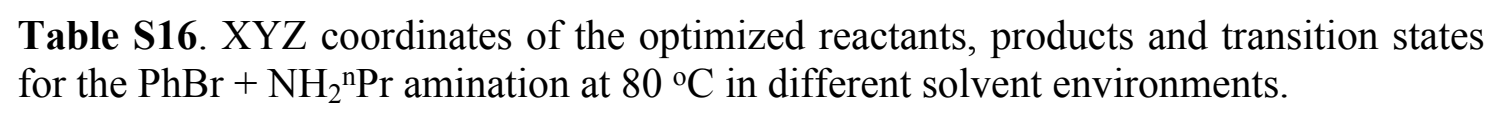 & S37 \\
\hline Full Gaussian 09 Reference & S40 \\
\hline
\end{tabular}



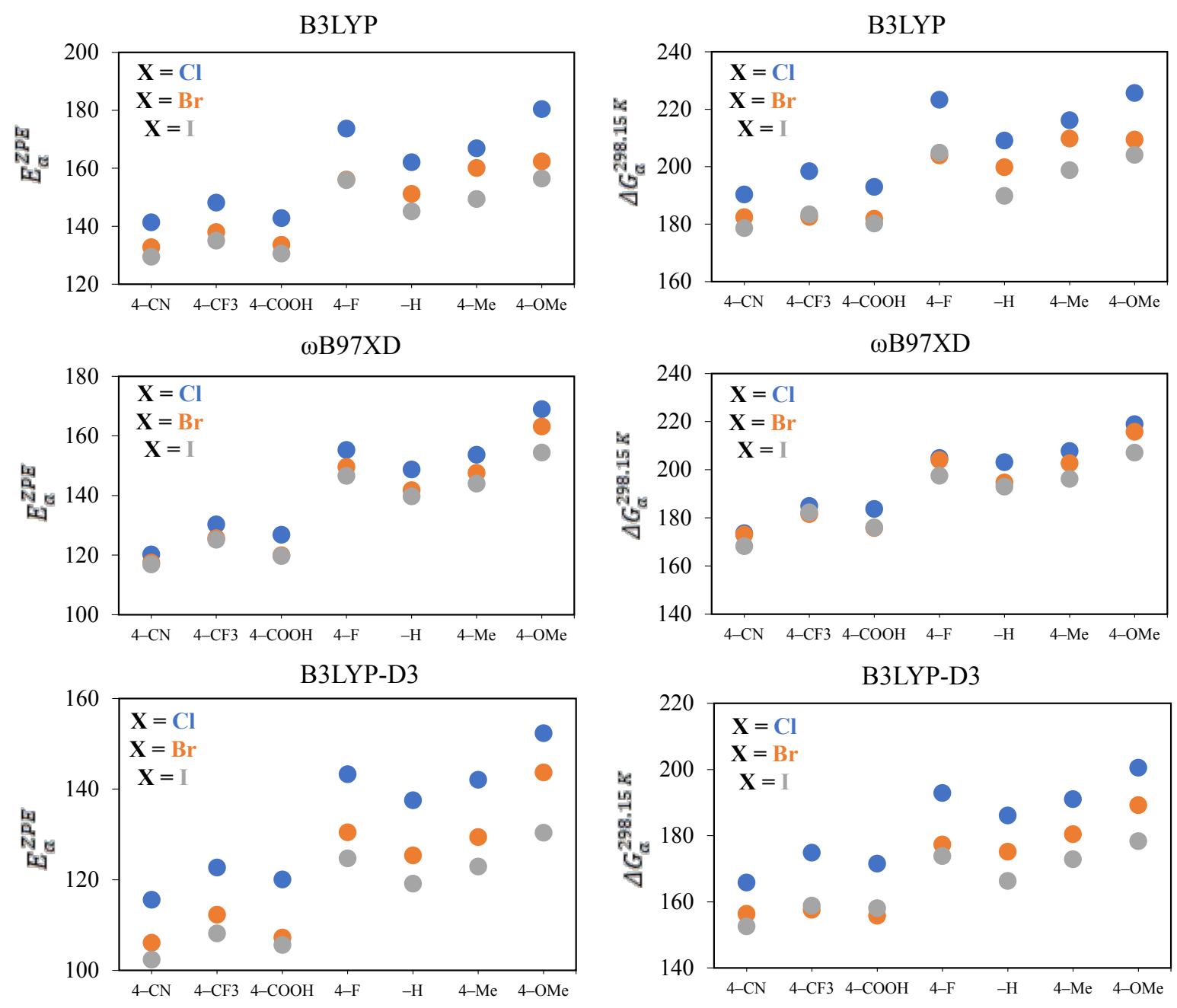

Figure S1. Zero-point corrected and free energy barrier heights using the different functionals for the non-catalyzed 4- $\mathrm{RPhX}+\mathrm{NH}_{2}{ }^{\mathrm{n}} \mathrm{Pr}$ amination, where $\mathrm{R}=-\mathrm{H},-\mathrm{Me},-\mathrm{OMe},-\mathrm{F},-\mathrm{CF}_{3},-\mathrm{COOH}$; and $\mathrm{X}=-\mathrm{Cl},-\mathrm{Br}$, and $-\mathrm{I}$. 
Table S1. Zero-point corrected energy $\left(E_{a}^{Z P E}\right)$ and free energy $\left(\Delta G_{a}^{298.15 K}\right)$ changes in $\mathrm{kJ} \mathrm{mol}^{-1}$ for the $n-\mathrm{RPhX}+\mathrm{NH}_{2}{ }^{\mathrm{n}} \mathrm{Pr}$ amination for $\mathrm{X}=\mathrm{Cl}$, where $\mathrm{n}=2$ (ortho), 3 (meta) or 4 (para) and $\mathrm{R}=$ $-\mathrm{H},-\mathrm{Me},-\mathrm{OMe},-\mathrm{F},-\mathrm{CF}_{3},-\mathrm{COOH}$, and $-\mathrm{CN}$.

\begin{tabular}{|c|c|c|c|c|c|c|}
\hline \multirow[b]{2}{*}{$\mathbf{R}$} & \multicolumn{3}{|c|}{ Energies with ZPE Corrections } & \multicolumn{3}{|c|}{ Free Energies } \\
\hline & $\begin{array}{l}\text { Reactant - } \\
\text { Product }\end{array}$ & $\begin{array}{c}\text { TS - } \\
\text { Reactant }\end{array}$ & $\begin{array}{c}\text { TS - } \\
\text { Product }\end{array}$ & $\begin{array}{c}\text { Reactant - } \\
\text { Product }\end{array}$ & $\begin{array}{c}\text { TS - } \\
\text { Reactant }\end{array}$ & $\begin{array}{c}\text { TS - } \\
\text { Product }\end{array}$ \\
\hline $2-\mathrm{CF}_{3}$ & 50.38 & 139.62 & 189.95 & 40.50 & 190.05 & 230.48 \\
\hline $2-\mathrm{CN}$ & 57.44 & 133.90 & 191.29 & 49.77 & 182.91 & 232.61 \\
\hline $2-\mathrm{COOH}$ & 62.33 & 120.61 & 182.90 & 51.26 & 173.84 & 225.03 \\
\hline $2-\mathrm{F}$ & 41.92 & 156.22 & 198.08 & 32.27 & 204.38 & 236.58 \\
\hline 2-Me & 27.85 & 166.17 & 193.95 & 16.99 & 215.25 & 232.16 \\
\hline 2-OMe & 38.20 & 163.90 & 202.03 & 27.51 & 212.03 & 239.46 \\
\hline $3-\mathrm{CF}_{3}$ & 38.13 & 154.10 & 192.18 & 33.70 & 196.97 & 230.59 \\
\hline $3-\mathrm{CN}$ & 40.76 & 151.29 & 191.99 & 30.24 & 200.49 & 230.65 \\
\hline $3-\mathrm{COOH}$ & 35.80 & 158.33 & 194.06 & 25.18 & 206.94 & 232.04 \\
\hline $3-\mathrm{F}$ & 35.83 & 156.43 & 192.20 & 25.75 & 205.13 & 230.80 \\
\hline 3-Me & 29.19 & 163.19 & 192.32 & 19.43 & 212.94 & 232.29 \\
\hline 3-OMe & 30.09 & 161.86 & 191.89 & 19.63 & 210.42 & 229.97 \\
\hline $4-\mathrm{CF}_{3}$ & 41.02 & 148.14 & 189.11 & 29.80 & 198.46 & 228.18 \\
\hline 4-CN & 45.96 & 141.36 & 187.27 & 36.01 & 190.31 & 226.25 \\
\hline $4-\mathrm{COOH}$ & 43.26 & 142.83 & 186.04 & 32.99 & 192.98 & 225.90 \\
\hline 4-F & 29.04 & 173.71 & 202.68 & 18.53 & 223.30 & 241.75 \\
\hline 4-Me & 27.11 & 166.90 & 193.95 & 15.09 & 216.15 & 231.16 \\
\hline 4-OMe & 22.94 & 180.39 & 203.27 & 12.15 & 225.69 & 237.75 \\
\hline $\mathrm{H}$ & 30.17 & 162.09 & 192.20 & 20.14 & 209.08 & 229.14 \\
\hline
\end{tabular}


Table S2. Zero-point corrected energy $\left(E_{a}^{Z P E}\right)$ and free energy $\left(\Delta G_{a}^{298.15 K}\right)$ changes in kJ mol-1 for the $n-\mathrm{RPhX}+\mathrm{NH}_{2}{ }^{\mathrm{n}} \mathrm{Pr}$ amination for $\mathrm{X}=\mathrm{Br}$, where $\mathrm{n}=2$ (ortho), 3 (meta) or 4 (para) and $\mathrm{R}=$ $-\mathrm{H},-\mathrm{Me},-\mathrm{OMe},-\mathrm{F},-\mathrm{CF}_{3},-\mathrm{COOH}$, and $-\mathrm{CN}$.

\begin{tabular}{|c|c|c|c|c|c|c|}
\hline & \multicolumn{3}{|c|}{ Energies with ZPE Corrections } & \multicolumn{3}{|c|}{ Free Energies } \\
\hline $\mathbf{R}$ & $\begin{array}{c}\text { Reactant - } \\
\text { Product }\end{array}$ & $\begin{array}{c}\text { TS - } \\
\text { Reactant }\end{array}$ & $\begin{array}{c}\text { TS - } \\
\text { Product }\end{array}$ & $\begin{array}{c}\text { Reactant - } \\
\text { Product }\end{array}$ & $\begin{array}{c}\text { TS - } \\
\text { Reactant }\end{array}$ & $\begin{array}{c}\text { TS - } \\
\text { Product }\end{array}$ \\
\hline $2-\mathrm{CF}_{3}$ & 51.08 & 129.82 & 180.85 & 42.23 & 179.86 & 222.02 \\
\hline $2-\mathrm{CN}$ & 57.06 & 126.50 & 183.51 & 50.07 & 176.44 & 226.44 \\
\hline $2-\mathrm{COOH}$ & 63.10 & 112.19 & 175.25 & 52.95 & 163.78 & 216.67 \\
\hline $2-\mathrm{F}$ & 38.95 & 146.09 & 184.99 & 29.98 & 194.42 & 224.32 \\
\hline 2-Me & 27.26 & 152.86 & 180.06 & 16.97 & 201.57 & 218.46 \\
\hline 2-OMe & 35.55 & 152.17 & 187.66 & 25.63 & 199.62 & 225.17 \\
\hline $3-\mathrm{CF}_{3}$ & 36.87 & 143.75 & 180.57 & 26.88 & 192.70 & 219.50 \\
\hline $3-\mathrm{CN}$ & 39.60 & 141.45 & 181.00 & 29.69 & 189.85 & 219.47 \\
\hline $3-\mathrm{COOH}$ & 34.41 & 147.49 & 181.84 & 24.36 & 195.18 & 219.47 \\
\hline $3-\mathrm{F}$ & 34.54 & 145.96 & 180.45 & 25.08 & 194.79 & 219.80 \\
\hline 3-Me & 27.99 & 151.46 & 179.39 & 20.25 & 199.21 & 219.38 \\
\hline 3-OMe & 28.51 & 150.62 & 179.08 & 18.52 & 198.43 & 216.87 \\
\hline $4-\mathrm{CF}_{3}$ & 39.99 & 138.01 & 177.95 & 29.40 & 182.54 & 211.87 \\
\hline $4-\mathrm{CN}$ & 45.06 & 132.80 & 177.81 & 35.71 & 182.46 & 218.10 \\
\hline 4-COOH & 42.34 & 133.62 & 175.91 & 32.70 & 181.89 & 214.52 \\
\hline 4-F & 27.47 & 156.09 & 183.50 & 17.57 & 203.88 & 221.37 \\
\hline 4-Me & 25.58 & 160.08 & 185.60 & 14.87 & 209.82 & 224.61 \\
\hline 4-OMe & 21.06 & 162.35 & 183.34 & 10.95 & 209.43 & 220.30 \\
\hline $\mathrm{H}$ & 28.86 & 151.19 & 180.00 & 19.41 & 199.86 & 219.19 \\
\hline
\end{tabular}


Table S3. Zero-point corrected energy $\left(E_{a}^{Z P E}\right)$ and free energy $\left(\Delta G_{a}^{298.15 K}\right)$ changes in kJ mol-1 for the $n-\mathrm{RPhX}+\mathrm{NH}_{2}{ }^{\mathrm{n}} \mathrm{Pr}$ amination for $\mathrm{X}=\mathrm{I}$, where $\mathrm{n}=2$ (ortho), 3 (meta) or 4 (para) and $\mathrm{R}=$ $-\mathrm{H},-\mathrm{Me},-\mathrm{OMe},-\mathrm{F},-\mathrm{CF}_{3},-\mathrm{COOH}$, and $-\mathrm{CN}$.

\begin{tabular}{|c|c|c|c|c|c|c|}
\hline \multirow[b]{2}{*}{$\mathbf{R}$} & \multicolumn{3}{|c|}{ Energies with ZPE Corrections } & \multicolumn{3}{|c|}{ Free Energies } \\
\hline & $\begin{array}{l}\text { Reactant - } \\
\text { Product }\end{array}$ & $\begin{array}{c}\text { TS - } \\
\text { Reactant }\end{array}$ & $\begin{array}{c}\text { TS - } \\
\text { Product }\end{array}$ & $\begin{array}{c}\text { Reactant - } \\
\text { Product }\end{array}$ & $\begin{array}{c}\text { TS - } \\
\text { Reactant }\end{array}$ & $\begin{array}{c}\text { TS - } \\
\text { Product }\end{array}$ \\
\hline $2-\mathrm{CF}_{3}$ & 49.33 & 125.95 & 175.23 & 40.51 & 176.50 & 216.95 \\
\hline $2-\mathrm{CN}$ & 54.01 & 123.51 & 177.48 & 47.24 & 172.12 & 219.29 \\
\hline $2-\mathrm{COOH}$ & 61.09 & 108.99 & 170.04 & 51.56 & 159.76 & 211.26 \\
\hline $2-\mathrm{F}$ & 32.90 & 142.04 & 174.88 & 24.13 & 189.55 & 213.61 \\
\hline 2-Me & 25.83 & 145.07 & 170.84 & 15.96 & 192.69 & 208.58 \\
\hline 2-OMe & 30.09 & 147.07 & 177.11 & 20.39 & 195.22 & 215.53 \\
\hline $3-\mathrm{CF}_{3}$ & 33.76 & 139.72 & 173.43 & 23.10 & 189.00 & 212.02 \\
\hline $3-\mathrm{CN}$ & 36.43 & 137.77 & 174.15 & 26.72 & 186.19 & 212.84 \\
\hline $3-\mathrm{COOH}$ & 31.52 & 143.12 & 174.58 & 21.71 & 191.24 & 212.87 \\
\hline $3-\mathrm{F}$ & 31.40 & 141.45 & 172.80 & 22.13 & 189.51 & 211.57 \\
\hline 3-Me & 24.74 & 146.30 & 170.98 & 20.48 & 190.19 & 210.60 \\
\hline 3-OMe & 25.86 & 145.27 & 171.08 & 16.05 & 193.15 & 209.13 \\
\hline $4-\mathrm{CF}_{3}$ & 37.23 & 135.07 & 172.25 & 26.55 & 183.42 & 209.90 \\
\hline $4-\mathrm{CN}$ & 42.25 & 129.45 & 171.66 & 33.08 & 178.60 & 211.62 \\
\hline 4-COOH & 39.79 & 130.57 & 170.32 & 30.30 & 180.25 & 210.48 \\
\hline 4-F & 24.27 & 155.92 & 180.14 & 14.55 & 204.90 & 219.38 \\
\hline $4-\mathrm{Me}$ & 22.71 & 149.41 & 172.06 & 11.87 & 198.81 & 210.60 \\
\hline 4-OMe & 17.81 & 156.49 & 174.24 & 7.98 & 204.13 & 212.03 \\
\hline $\mathrm{H}$ & 26.23 & 145.10 & 171.27 & 26.23 & 145.10 & 171.27 \\
\hline
\end{tabular}


Table S4. Barrier heights $\left(E_{a}^{Z P E}\right.$ and $\triangle G_{a}^{298.15 K}$ ) for the amination reactions calculated at the B3LYP-D3/cc-pVTZ level of theory $\left(n-\mathrm{RPhX}+\mathrm{NH}_{2} \mathrm{R}^{\prime} \rightarrow n\right.$-RPhNHR' $\left.+\mathrm{HX}\right)$, where $n=4$ (para); $\mathrm{R}=-\mathrm{CF}_{3},-\mathrm{CN},-\mathrm{COOH},-\mathrm{F},-\mathrm{Me},-\mathrm{OMe}$, and $-\mathrm{H} ; \mathrm{X}=-\mathrm{Cl},-\mathrm{Br}$, and $-\mathrm{I}$; and $\mathrm{R}^{\prime}=-{ }^{\mathrm{n}} \mathrm{Pr}$.

\begin{tabular}{ccccccc}
\hline & \multicolumn{3}{c}{$E_{a}^{Z P E} / \mathrm{kJ} \mathrm{mol}^{-1}$} & \multicolumn{3}{c}{$\Delta G_{a}^{298.15 K / \mathrm{kJ} \mathrm{mol}^{-1}}$} \\
\hline$n-\mathrm{R}$ & $\mathrm{X}=\mathrm{Cl}$ & $\mathrm{X}=\mathrm{Br}$ & $\mathrm{X}=\mathrm{I}$ & $\mathrm{X}=\mathrm{Cl}$ & $\mathrm{X}=\mathrm{Br}$ & $\mathrm{X}=\mathrm{I}$ \\
\hline $4-\mathrm{CN}$ & 115.56 & 106.07 & 102.33 & 165.82 & 156.37 & 152.59 \\
$4-\mathrm{CF}_{3}$ & 122.67 & 112.26 & 108.15 & 174.86 & 157.54 & 158.85 \\
$4-\mathrm{COOH}$ & 120.04 & 107.20 & 105.59 & 171.53 & 155.76 & 158.06 \\
$4-\mathrm{F}$ & 143.26 & 130.43 & 124.69 & 192.86 & 177.28 & 173.82 \\
$-\mathrm{H}$ & 137.53 & 125.36 & 119.13 & 186.07 & 175.15 & 166.30 \\
$4-\mathrm{Me}$ & 142.02 & 129.40 & 122.88 & 190.99 & 180.39 & 172.85 \\
$4-\mathrm{OMe}$ & 152.31 & 143.68 & 130.34 & 200.51 & 189.20 & 178.29 \\
\hline
\end{tabular}

Table S5. Barrier heights $\left(E_{a}^{Z P E}\right.$ and $\Delta G_{a}^{298.15 K}$ ) for the amination reactions calculated at the $\omega \mathrm{B} 97 \mathrm{XD} / \mathrm{cc}-\mathrm{pVTZ}$ level of theory $\left(n-\mathrm{RPhX}+\mathrm{NH}_{2} \mathrm{R}^{\prime} \rightarrow n\right.$-RPhNHR' $\left.+\mathrm{HX}\right)$, where $n=4$ (para); $\mathrm{R}=-\mathrm{CF}_{3},-\mathrm{CN},-\mathrm{COOH},-\mathrm{F},-\mathrm{Me},-\mathrm{OMe}$, and $-\mathrm{H} ; \mathrm{X}=-\mathrm{Cl},-\mathrm{Br}$, and $-\mathrm{I}$; and $\mathrm{R}^{\prime}=-{ }^{\mathrm{n}} \mathrm{Pr}$.

\begin{tabular}{ccccccc}
\hline & \multicolumn{3}{c}{$E_{a}^{Z P E} / \mathrm{kJ} \mathrm{mol}^{-1}$} & \multicolumn{3}{c}{$\Delta G_{a}^{298.15 K} / \mathrm{kJ} \mathrm{mol}^{-1}$} \\
\hline$n-\mathrm{R}$ & $\mathrm{X}=\mathrm{Cl}$ & $\mathrm{X}=\mathrm{Br}$ & $\mathrm{X}=\mathrm{I}$ & $\mathrm{X}=\mathrm{Cl}$ & $\mathrm{X}=\mathrm{Br}$ & $\mathrm{X}=\mathrm{I}$ \\
\hline $4-\mathrm{CN}$ & 120.24 & 117.52 & 116.89 & 173.59 & 172.95 & 168.22 \\
$4-\mathrm{CF}_{3}$ & 130.31 & 125.68 & 125.20 & 184.89 & 181.52 & 182.27 \\
$4-\mathrm{COOH}$ & 126.82 & 119.93 & 119.73 & 183.61 & 175.70 & 175.95 \\
$4-\mathrm{F}$ & 155.27 & 149.62 & 146.58 & 204.82 & 203.99 & 197.43 \\
$-\mathrm{H}$ & 148.76 & 141.77 & 139.71 & 203.05 & 194.63 & 192.88 \\
$4-\mathrm{Me}$ & 153.66 & 147.60 & 143.98 & 207.72 & 202.64 & 196.10 \\
$4-\mathrm{OMe}$ & 168.95 & 163.12 & 154.42 & 218.89 & 215.74 & 207.08 \\
\hline
\end{tabular}


The Effect of the Amine Substituent. The $4-\mathrm{MePhBr}+\mathrm{NH}_{2} \mathrm{R}^{\prime}$ amination was investigated to determine the influence of the alkyl character on the barrier heights of the reaction (Table S11) where $\mathrm{R}^{\prime}=$ methyl $(-\mathrm{Me})$, ethylamine $(-\mathrm{Et})$, n-propylamine $\left({ }^{\mathrm{n}} \mathrm{Pr}\right)$, and $\mathrm{n}$-butylamine $\left(-^{\mathrm{n}} \mathrm{Bu}\right)$. The barrier heights increased by $\sim 7 \mathrm{~kJ} \mathrm{~mol}^{-1}$ as the carbon chain length increased from $-\mathrm{Me}$ to ${ }^{\mathrm{n}} \mathrm{Pr}$, but then decreased noticeably from ${ }^{\mathrm{n}} \mathrm{Pr}$ to ${ }^{\mathrm{n}} \mathrm{Bu}$. The basis for this decline in energy is not clear. The $\mathrm{R}^{\prime}$ chain extended away from the bonding region between reactants in the transition structures, rather than toward the ring, so steric effects due to long $\mathrm{R}^{\prime}$ groups are unlikely to have any significant influence on the reaction. And the alkyl chain length appears indeed to have a rather weak influence on the nature and height of the reaction barrier despite the anomaly noted above. Increasing the alkyl chain length is expected to increase the nucleophilicity (lone pair availability) of the amine, but it does not appear to do so to a sufficiently significant extent as to dramatically modify the barrier height, and a similar weak response is predicted by the computed free energies as well.

Table S6. Zero-point corrected energy $\left(E_{a}^{Z P E}\right)$ and free energy $\left(\Delta G_{a}^{298.15 K}\right)$ changes in $\mathrm{kJ} \mathrm{mol}^{-1}$ for the non-catalyzed 4-MePhBr $+\mathrm{NH}_{2} \mathrm{R}$ amination, where $\mathrm{R}^{\prime}=-\mathrm{Me}$, - $\mathrm{Et}$, $-{ }^{\mathrm{n}} \mathrm{Pr}$, and $-{ }^{\mathrm{n}} \mathrm{Bu}$.

\begin{tabular}{c|ccc|ccc}
\hline & \multicolumn{2}{|c|}{ Energies with ZPE Corrections } & \multicolumn{3}{c}{ Free Energies } \\
\hline R' & $\begin{array}{c}\text { Reactant }- \\
\text { Product }\end{array}$ & $\begin{array}{c}\text { TS }- \\
\text { Reactant }\end{array}$ & $\begin{array}{c}\text { TS }- \\
\text { Product }\end{array}$ & $\begin{array}{c}\text { Reactant }- \\
\text { Product }\end{array}$ & $\begin{array}{c}\text { TS - } \\
\text { Reactant }\end{array}$ & $\begin{array}{c}\text { TS - } \\
\text { Product }\end{array}$ \\
\hline${ }^{\mathrm{n}} \mathrm{Bu}$ & 26.04 & 153.65 & 179.63 & 15.02 & 194.25 & 209.19 \\
$-\mathrm{n} P r$ & 25.58 & 160.08 & 185.60 & 14.58 & 209.82 & 224.32 \\
$-\mathrm{Et}$ & 25.86 & 155.24 & 181.05 & 15.91 & 200.85 & 216.68 \\
$-\mathrm{Me}$ & 27.32 & 153.68 & 180.94 & 13.55 & 200.44 & 213.91
\end{tabular}


Table S7. Zero-point corrected energy $\left(E_{a}^{Z P E}\right)$ and free energy $\left(\Delta G_{a}^{298.15 K}\right)$ changes in $\mathrm{kJ} \mathrm{mol}^{-1}$ for the $4-\mathrm{MePhCl}+\mathrm{NH}_{2}{ }^{\mathrm{n}} \mathrm{Pr}$ amination at different temperatures. The default used for thermochemistry analysis is $25^{\circ} \mathrm{C}$.

\begin{tabular}{c|ccc|ccc}
\hline & \multicolumn{2}{|c|}{ Energies with ZPE Corrections } & \multicolumn{3}{c}{ Free Energies } \\
\hline \multirow{2}{*}{ Temperature } & $\begin{array}{c}\text { Reactant }- \\
\text { Product }\end{array}$ & $\begin{array}{c}\text { TS }- \\
\text { Reactant }\end{array}$ & $\begin{array}{c}\text { TS - } \\
\text { Product }\end{array}$ & $\begin{array}{c}\text { Reactant - } \\
\text { Product }\end{array}$ & $\begin{array}{c}\text { TS - } \\
\text { Reactant }\end{array}$ & $\begin{array}{c}\text { TS - } \\
\text { Product }\end{array}$ \\
\hline-20 & 27.08 & 166.80 & 193.82 & 16.42 & 208.23 & 224.57 \\
25 & 27.11 & 166.90 & 193.95 & 15.09 & 216.15 & 231.16 \\
80 & 27.08 & 166.88 & 193.90 & 13.36 & 224.83 & 238.10
\end{tabular}

Table S8. Zero-point corrected energy $\left(E_{a}^{Z P E}\right)$ and free energy $\left(\Delta G_{a}^{298.15 K}\right)$ changes in $\mathrm{kJ}^{\mathrm{mol}}{ }^{-1}$ for the $4-\mathrm{MePhBr}+\mathrm{NH}_{2}{ }^{\mathrm{n}} \mathrm{Pr}$ amination at different temperatures. The default used for thermochemistry analysis is $25^{\circ} \mathrm{C}$.

\begin{tabular}{c|ccc|ccc}
\hline & \multicolumn{2}{|c|}{ Energies with ZPE Corrections } & \multicolumn{3}{c}{ Free Energies } \\
\hline \multirow{2}{*}{ Temperature } & $\begin{array}{c}\text { Reactant }- \\
\text { Product }\end{array}$ & $\begin{array}{c}\text { TS - } \\
\text { Reactant }\end{array}$ & $\begin{array}{c}\text { TS - } \\
\text { Product }\end{array}$ & $\begin{array}{c}\text { Reactant - } \\
\text { Product }\end{array}$ & $\begin{array}{c}\text { TS - } \\
\text { Reactant }\end{array}$ & $\begin{array}{c}\text { TS - } \\
\text { Product }\end{array}$ \\
\hline-20 & 25.55 & 155.26 & 180.74 & 15.54 & 197.39 & 212.86 \\
25 & 25.58 & 160.08 & 185.60 & 14.87 & 209.82 & 224.61 \\
80 & 25.55 & 155.26 & 180.74 & 12.50 & 214.25 & 226.67
\end{tabular}


Table S9. Zero-point corrected energy $\left(E_{a}^{Z P E}\right)$ and free energy $\left(\Delta G_{a}^{298.15 K}\right)$ changes in $\mathrm{kJ} \mathrm{mol}^{-1}$ for the $\mathrm{PhBr}+\mathrm{NH}_{2}{ }^{\mathrm{n}} \mathrm{Pr}$ amination at $80{ }^{\circ} \mathrm{C}$ in different solvent environments.

\begin{tabular}{c|c|ccc|ccc}
\hline & & \multicolumn{2}{|c|}{ Energies with ZPE Corrections } & \multicolumn{3}{c}{ Free Energies } \\
\hline $\boldsymbol{\varepsilon}_{\boldsymbol{r}}$ & Solvent & $\begin{array}{c}\text { Reactant }- \\
\text { Product }\end{array}$ & $\begin{array}{c}\text { TS }- \\
\text { Reactant }\end{array}$ & $\begin{array}{c}\text { TS }- \\
\text { Product }\end{array}$ & $\begin{array}{c}\text { Reactant - } \\
\text { Product }\end{array}$ & $\begin{array}{c}\text { TS - } \\
\text { Reactant }\end{array}$ & $\begin{array}{c}\text { TS - } \\
\text { Product }\end{array}$ \\
\hline 1.00 & Vacuo & 28.86 & 151.19 & 180.00 & 18.19 & 235.70 & 253.80 \\
2.37 & Toluene & 29.38 & 143.31 & 172.63 & 18.64 & 200.27 & 218.84 \\
24.85 & Ethanol & 30.19 & 135.34 & 165.48 & 19.49 & 191.71 & 211.13 \\
35.00 & - & 30.23 & 135.03 & 165.20 & 19.55 & 191.36 & 210.84 \\
37.22 & DMF & 30.24 & 134.98 & 165.16 & 19.56 & 191.31 & 210.80 \\
50.00 & - & 30.26 & 134.79 & 165.00 & 19.59 & 191.10 & 210.62 \\
65.00 & - & 30.28 & 134.66 & 164.88 & 19.62 & 190.95 & 210.50 \\
78.36 & Water & 30.29 & 134.58 & 164.82 & 19.63 & 190.87 & 210.43
\end{tabular}


Table S10. Coordinates for the optimized reactants, products and transition states for the $n$ $\mathrm{RPhX}+\mathrm{NH}_{2}{ }^{\mathrm{n}} \mathrm{Pr}$ amination for $\mathrm{X}=\mathrm{Cl}$, where $n=2$ (ortho), 3 (meta) or 4 (para) and $\mathrm{R}=-\mathrm{H},-$ $\mathrm{Me},-\mathrm{OMe},-\mathrm{F},-\mathrm{CF}_{3},-\mathrm{COOH}$, and $-\mathrm{CN}$.

\begin{tabular}{|c|c|c|c|c|c|c|c|c|c|c|c|}
\hline \multicolumn{4}{|c|}{ Reactant } & \multicolumn{4}{|c|}{ Transition State } & & & roduct & \\
\hline & & & & & $\boldsymbol{n}=$ & 2, $\mathrm{R}=-$ & & & & & \\
\hline 6 & -3.758334000 & 1.615508000 & -0.037869000 & 6 & 2.091651000 & -2.222096000 & -0.000022000 & 6 & -3.675231000 & -0.423699000 & -0.257910000 \\
\hline 6 & -4.256249000 & 0.319417000 & -0.114197000 & 6 & 1.794192000 & -1.574391000 & -1.194919000 & 6 & -3.218231000 & 0.098034000 & 0.948436000 \\
\hline 6 & -2.387098000 & 1.826487000 & 0.054050000 & 6 & 1.794337000 & -1.574307000 & 1.194867000 & 6 & -2.752348000 & -0.860192000 & -1.198094000 \\
\hline 1 & -5.322132000 & 0.149006000 & -0.185763000 & 1 & 2.034538000 & -2.043642000 & -2.140823000 & 1 & -3.923236000 & 0.443623000 & 1.692702000 \\
\hline 1 & -1.995381000 & 2.833187000 & 0.113103000 & 1 & 2.034792000 & -2.043494000 & 2.140775000 & 1 & $\begin{array}{l}-3.091613000 \\
\end{array}$ & -1.266544000 & -2.141680000 \\
\hline 6 & -3.389744000 & -0.767745000 & -0.099046000 & 6 & 1.204082000 & -0.318125000 & -1.212515000 & 6 & -1.859681000 & 0.180641000 & 1.208086000 \\
\hline 6 & -1.503517000 & 0.752144000 & 0.070886000 & 6 & 1.204223000 & -0.318042000 & 1.212443000 & 6 & -1.386899000 & -0.783270000 & -0.946326000 \\
\hline 1 & -3.762444000 & -1.779771000 & -0.157467000 & 1 & 1.021926000 & 0.187989000 & -2.150339000 & 1 & -1.512339000 & 0.595856000 & 2.146537000 \\
\hline 1 & -0.433355000 & 0.899538000 & 0.140888000 & 1 & 1.022180000 & 0.188139000 & 2.150254000 & 1 & -0.688098000 & -1.123306000 & -1.696071000 \\
\hline 6 & -2.025742000 & -0.530400000 & -0.006306000 & 6 & 0.911939000 & 0.324212000 & -0.000041000 & 6 & -0.928040000 & -0.260728000 & 0.263545000 \\
\hline 17 & -0.934015000 & -1.912495000 & 0.012956000 & 17 & 1.467149000 & 2.331919000 & -0.000143000 & 17 & 1.365566000 & 2.681016000 & -0.408848000 \\
\hline 1 & -4.436894000 & 2.457571000 & -0.050200000 & 1 & 2.556370000 & -3.197280000 & -0.000016000 & 1 & -4.735309000 & -0.487211000 & -0.460011000 \\
\hline 6 & 3.377861000 & 0.738883000 & -0.003256000 & 6 & -1.825982000 & -0.182352000 & -0.000050000 & 6 & 1.421850000 & -1.006590000 & -0.111013000 \\
\hline 1 & 3.348296000 & 1.211605000 & -0.988782000 & 1 & -1.613567000 & -0.795842000 & -0.876452000 & 1 & 1.067563000 & -2.043479000 & -0.082551000 \\
\hline 1 & 3.549645000 & 1.546031000 & 0.714240000 & 1 & -1.613565000 & -0.795973000 & 0.876262000 & 1 & 1.505326000 & -0.720584000 & -1.161106000 \\
\hline 6 & 4.550099000 & -0.245627000 & 0.052057000 & 6 & -3.279635000 & 0.284768000 & -0.000019000 & 6 & 2.790142000 & -0.899320000 & 0.552276000 \\
\hline 1 & 4.373350000 & -1.049229000 & -0.669550000 & 1 & -3.459074000 & 0.913987000 & -0.876232000 & 1 & 2.698758000 & -1.172468000 & 1.608704000 \\
\hline 1 & 4.569645000 & -0.718849000 & 1.038839000 & 1 & -3.458870000 & 0.914457000 & 0.875900000 & 1 & 3.121098000 & 0.140594000 & 0.522447000 \\
\hline 6 & 5.898615000 & 0.415517000 & -0.230150000 & 6 & -4.258007000 & -0.889780000 & 0.000429000 & 6 & 3.829570000 & -1.795765000 & -0.117170000 \\
\hline 1 & 5.915383000 & 0.871215000 & -1.222412000 & 1 & -4.119820000 & -1.519088000 & -0.880247000 & 1 & 3.533777000 & -2.845925000 & -0.080775000 \\
\hline 1 & 6.112544000 & 1.202015000 & 0.496594000 & 1 & -4.120883000 & -1.517519000 & 0.882385000 & 1 & 3.967673000 & -1.524050000 & -1.165002000 \\
\hline 1 & 6.714510000 & -0.306640000 & -0.184880000 & 1 & -5.289577000 & -0.538499000 & -0.000514000 & 1 & 4.796841000 & -1.706578000 & 0.376898000 \\
\hline 7 & 2.051798000 & 0.179261000 & 0.262556000 & 7 & -0.848201000 & 0.913789000 & 0.000032000 & 7 & 0.453502000 & -0.111145000 & 0.539225000 \\
\hline 1 & 2.020168000 & -0.249490000 & 1.180586000 & 1 & -0.909672000 & 1.514051000 & 0.816611000 & 1 & 0.928438000 & 1.486362000 & 0.027282000 \\
\hline 1 & 1.826989000 & -0.553711000 & -0.400361000 & 1 & -0.909712000 & 1.514249000 & -0.816400000 & 1 & 0.615197000 & -0.070218000 & 1.537499000 \\
\hline & & & & & $n=2$ & D - & & & & & \\
\hline 6 & 3.935505000 & 0.974004000 & -0.886721000 & 6 & -2.021360000 & -2.287028000 & -0.172904000 & 6 & -3.498956000 & -0.802646000 & -0.526230000 \\
\hline 6 & 2.978541000 & 1.518740000 & -0.040936000 & 6 & -1.811585000 & -1.549726000 & 0.987301000 & 6 & -3.142094000 & 0.091246000 & 0.477379000 \\
\hline 6 & 3.895294000 & -0.379069000 & -1.196638000 & 6 & -1.626800000 & -1.740458000 & -1.387232000 & 6 & -2.504188000 & -1.474306000 & -1.218891000 \\
\hline 1 & 3.015361000 & 2.573731000 & 0.198936000 & 1 & -2.124574000 & -1.955467000 & 1.942253000 & 1 & -3.914833000 & 0.621913000 & 1.019458000 \\
\hline 1 & 4.635323000 & -0.816767000 & -1.852642000 & 1 & -1.793071000 & -2.278577000 & -2.311736000 & 1 & -2.759652000 & -2.170919000 & -2.006025000 \\
\hline 6 & 1.957541000 & 0.746767000 & 0.519429000 & 6 & -1.216641000 & -0.289391000 & 0.985117000 & 6 & -1.813005000 & 0.333162000 & 0.806825000 \\
\hline 6 & 2.895383000 & -1.177048000 & -0.657154000 & 6 & -1.030171000 & -0.488125000 & -1.441694000 & 6 & -1.167260000 & -1.258215000 & -0.906207000 \\
\hline 1 & 2.844751000 & -2.232321000 & -0.882608000 & 1 & -0.768990000 & -0.046914000 & -2.393334000 & 1 & -0.403766000 & -1.787031000 & -1.456788000 \\
\hline 6 & 1.948846000 & -0.609442000 & 0.184140000 & 6 & -0.840066000 & 0.236631000 & -0.262441000 & 6 & -0.811800000 & -0.362084000 & 0.101406000 \\
\hline 17 & 0.702411000 & -1.665680000 & 0.845843000 & 17 & -1.383435000 & 2.268295000 & -0.523611000 & 17 & 1.390522000 & 2.469090000 & -1.096560000 \\
\hline 1 & 4.710516000 & 1.604941000 & -1.300452000 & 1 & -2.490711000 & -3.259171000 & -0.128021000 & 1 & -4.541200000 & -0.966926000 & -0.762404000 \\
\hline 6 & -3.621064000 & 0.710321000 & 0.019207000 & 6 & 1.930713000 & -0.204595000 & -0.016487000 & 6 & 1.583492000 & -1.059490000 & 0.013197000 \\
\hline 1 & -3.688530000 & 0.535924000 & 1.096493000 & 1 & 1.725722000 & -0.670417000 & 0.948161000 & 1 & 1.271154000 & -2.078945000 & 0.267073000 \\
\hline 1 & -3.946711000 & 1.741897000 & -0.141146000 & 1 & 1.727928000 & -0.957227000 & -0.780027000 & 1 & 1.707801000 & -1.019465000 & -1.070675000 \\
\hline 6 & -4.576003000 & -0.241014000 & -0.708876000 & 6 & 3.381241000 & 0.268227000 & -0.091891000 & 6 & 2.910836000 & -0.734470000 & 0.688925000 \\
\hline 1 & -4.247439000 & -1.271198000 & -0.540625000 & 1 & 3.553256000 & 1.034909000 & 0.668693000 & 1 & 2.776820000 & -0.764493000 & 1.775285000 \\
\hline 1 & -4.497334000 & -0.066391000 & -1.786628000 & 1 & 3.554049000 & 0.747761000 & -1.059466000 & 1 & 3.202014000 & 0.286650000 & 0.435173000 \\
\hline 6 & -6.029705000 & -0.082365000 & -0.265099000 & 6 & 4.371719000 & -0.879854000 & 0.099183000 & 6 & 4.018345000 & -1.705709000 & 0.285867000 \\
\hline 1 & -6.140487000 & -0.280677000 & 0.803054000 & 1 & 4.239544000 & -1.358634000 & 1.070932000 & 1 & 3.762371000 & -2.734111000 & 0.547753000 \\
\hline 1 & -6.390376000 & 0.931641000 & -0.450229000 & 1 & 4.242768000 & -1.645061000 & -0.668108000 & 1 & 4.200547000 & -1.670717000 & -0.789515000 \\
\hline 1 & -6.687607000 & -0.769490000 & -0.798309000 & 1 & 5.399601000 & -0.522349000 & 0.042405000 & 1 & 4.954113000 & -1.459127000 & 0.787110000 \\
\hline 7 & -2.209687000 & 0.623478000 & -0.358291000 & 7 & 0.944521000 & 0.865447000 & -0.202313000 & 7 & 0.549025000 & -0.089442000 & 0.398573000 \\
\hline 1 & -2.093084000 & 0.803210000 & -1.348951000 & 1 & 1.029609000 & 1.346447000 & -1.091323000 & 1 & 0.988284000 & 1.366591000 & -0.433587000 \\
\hline 1 & -1.846161000 & -0.307376000 & -0.187111000 & 1 & 0.960731000 & 1.574651000 & 0.522310000 & 1 & 0.661646000 & 0.186382000 & 1.365419000 \\
\hline 6 & 0.928598000 & 1.362885000 & 1.423433000 & 6 & -1.035197000 & 0.484849000 & 2.260836000 & 6 & -1.456877000 & 1.321201000 & 1.885862000 \\
\hline 1 & -0.069307000 & 1.306575000 & 0.981949000 & 1 & 0.018252000 & 0.682923000 & 2.486744000 & 1 & -0.918746000 & 0.850433000 & 2.715819000 \\
\hline 1 & 0.886123000 & 0.846074000 & 2.383580000 & 1 & -1.535969000 & 1.452231000 & 2.203006000 & 1 & -0.825022000 & 2.126600000 & 1.504661000 \\
\hline 1 & 1.164183000 & 2.409764000 & 1.608686000 & 1 & -1.442520000 & $\begin{array}{r}-0.067449000 \\
\end{array}$ & 3.106367000 & 1 & -2.354540000 & 1.772584000 & 2.303952000 \\
\hline & & & & & & & & & & & \\
\hline 6 & -1.139301000 & -2.157315000 & 0.817808000 & 6 & 1.029565000 & 1.869852000 & -1.833592000 & 6 & -2.613525000 & -1.435343000 & -1.378561000 \\
\hline 6 & -0.756086000 & -0.836838000 & 1.030252000 & 6 & 0.909715000 & 1.914569000 & -0.440385000 & 6 & -2.697468000 & -0.729717000 & -0.179166000 \\
\hline 6 & -2.272734000 & -2.453452000 & 0.076170000 & 6 & 0.983518000 & 0.644534000 & -2.473096000 & 6 & -1.393315000 & -1.569390000 & -2.017710000 \\
\hline 1 & 0.131827000 & -0.624263000 & 1.605028000 & 1 & 0.931280000 & 2.868404000 & 0.066771000 & 1 & -3.651690000 & -0.628260000 & 0.314877000 \\
\hline 1 & -2.573034000 & -3.478507000 & -0.089048000 & 1 & 1.083661000 & 0.582583000 & -3.548759000 & 1 & -1.320301000 & -2.112488000 & -2.949634000 \\
\hline 6 & -1.507423000 & 0.215083000 & 0.501456000 & 6 & 0.757000000 & 0.754354000 & 0.298433000 & 6 & -1.558658000 & -0.162294000 & 0.373747000 \\
\hline 6 & -3.029303000 & -1.415053000 & -0.457180000 & 6 & 0.834603000 & -0.536887000 & -1.745061000 & 6 & -0.249463000 & -0.998535000 & -1.465085000 \\
\hline 1 & -3.917222000 & -1.618129000 & -1.038366000 & 1 & 0.864548000 & -1.493465000 & -2.247796000 & 1 & 0.695406000 & -1.099512000 & -1.979011000 \\
\hline 6 & -2.652911000 & -0.099797000 & -0.247751000 & 6 & 0.754979000 & -0.496315000 & -0.355863000 & 6 & -0.311478000 & -0.292827000 & -0.270078000 \\
\hline 17 & -3.617302000 & 1.190772000 & -0.925858000 & 17 & 1.994004000 & -1.891503000 & 0.562539000 & 17 & 1.205496000 & 3.149614000 & -0.918869000 \\
\hline 6 & 3.697663000 & -0.465804000 & -0.633618000 & 6 & -2.000500000 & -1.178296000 & -0.284278000 & 6 & 2.146766000 & -0.215362000 & 0.180413000 \\
\hline 1 & 3.590806000 & -1.256156000 & 0.113886000 & 1 & -1.807704000 & -0.918038000 & -1.328984000 & 1 & 2.378466000 & -0.333637000 & -0.878046000 \\
\hline 1 & 3.961398000 & -0.962773000 & -1.578321000 & 1 & -2.630142000 & -2.071559000 & -0.278763000 & 1 & 2.844623000 & 0.534289000 & 0.557519000 \\
\hline 6 & 4.837309000 & 0.461371000 & -0.226030000 & 6 & -2.709027000 & -0.030686000 & 0.424837000 & 6 & 2.345808000 & -1.537338000 & 0.922751000 \\
\hline 1 & 4.579327000 & 0.935068000 & 0.723768000 & 1 & -2.063860000 & 0.848216000 & 0.414087000 & 1 & 1.619895000 & -2.268783000 & 0.562180000 \\
\hline 1 & 4.918376000 & 1.269519000 & -0.960383000 & 1 & -2.862644000 & -0.297807000 & 1.474718000 & 1 & 2.130897000 & -1.383697000 & 1.984234000 \\
\hline 6 & 6.179120000 & -0.259047000 & -0.111048000 & 6 & -4.050925000 & 0.300216000 & -0.224748000 & 6 & 3.762531000 & -2.084314000 & 0.755941000 \\
\hline 1 & 6.140353000 & -1.048208000 & 0.642673000 & 1 & -3.920623000 & 0.605633000 & -1.264181000 & 1 & 3.990928000 & -2.276190000 & -0.294274000 \\
\hline 1 & 6.465830000 & -0.721188000 & -1.057857000 & 1 & -4.723729000 & -0.559613000 & -0.211521000 & 1 & 4.507972000 & -1.381070000 & 1.132520000 \\
\hline 1 & 6.974577000 & 0.430156000 & 0.173852000 & 1 & -4.547039000 & 1.116428000 & 0.300008000 & 1 & 3.888707000 & -3.021739000 & 1.297791000 \\
\hline 7 & 2.423646000 & 0.259891000 & -0.698878000 & 7 & -0.696436000 & -1.495609000 & 0.318317000 & 7 & 0.802523000 & 0.367722000 & 0.301384000 \\
\hline 1 & 1.664448000 & -0.369451000 & -0.932180000 & 1 & -0.382425000 & -2.444272000 & 0.153698000 & 1 & 0.963327000 & 1.929792000 & -0.392177000 \\
\hline 1 & 2.454190000 & 0.957677000 & -1.434104000 & 1 & -0.643520000 & -1.312460000 & 1.315214000 & 1 & 0.591745000 & 0.621514000 & 1.258621000 \\
\hline 1 & -0.542134000 & -2.953943000 & 1.240893000 & 1 & 1.156006000 & 2.785380000 & -2.392981000 & 1 & -3.506596000 & -1.873160000 & -1.802303000 \\
\hline 8 & -1.212032000 & 1.523226000 & 0.658199000 & 8 & 0.570341000 & 0.699007000 & 1.657633000 & 8 & -1.528352000 & 0.543755000 & 1.545484000 \\
\hline
\end{tabular}




\begin{tabular}{|c|c|c|c|c|c|c|c|c|c|c|}
\hline 6 & -0.067241000 & 1.879516000 & 1.430584000 & 6 & 0.752330000 & 1.886884000 & 2.407018000 & 6 & -2.744219000 & 0.753404000 \\
\hline 1 & -0.166870000 & 1.530970000 & 2.461775000 & 1 & 1.750231000 & 2.303581000 & 2.250582000 & 1 & -3.461129000 & 1.307748000 \\
\hline 1 & 0.848964000 & 1.483383000 & 0.988060000 & 1 & 0.003842000 & 2.643918000 & 2.154732000 & 1 & -3.190397000 & -0.193054000 \\
\hline 1 & -0.040511000 & 2.965697000 & 1.422607000 & 1 & 0.638821000 & 1.606491000 & 3.451132000 & 1 & -2.488005000 & 1.341003000 \\
\hline & & & & & $n=$ & $2, \mathbf{R}=$ & & & & \\
\hline 6 & 1.723778000 & 2.291312000 & 0.053284000 & 6 & -2.048954000 & -2.242812000 & -0.153001000 & 6 & -3.525384000 & -0.538762000 \\
\hline 6 & 0.819417000 & 1.256885000 & -0.154388000 & 6 & -1.752233000 & -1.552237000 & 1.022247000 & 6 & -3.149970000 & -0.021885000 \\
\hline 6 & 3.069000000 & 2.015194000 & 0.271067000 & 6 & -1.718038000 & -1.657201000 & -1.367396000 & 6 & -2.547628000 & -0.949025000 \\
\hline 1 & -0.235916000 & 1.430697000 & -0.327441000 & 1 & -1.980593000 & -1.960245000 & 1.997832000 & 1 & -3.877765000 & 0.305568000 \\
\hline 1 & 3.772250000 & 2.820256000 & 0.432775000 & 1 & -1.947458000 & -2.164873000 & -2.295191000 & 1 & -2.829770000 & -1.351633000 \\
\hline 6 & 1.282547000 & -0.046746000 & -0.139686000 & 6 & -1.147388000 & -0.319621000 & 0.957465000 & 6 & -1.810230000 & 0.073099000 \\
\hline 6 & 3.520262000 & 0.701461000 & 0.282864000 & 6 & -1.111258000 & -0.406512000 & -1.430450000 & 6 & -1.198719000 & -0.847527000 \\
\hline 1 & 4.562470000 & 0.471934000 & 0.450820000 & 1 & -0.913866000 & 0.055702000 & -2.387685000 & 1 & -0.452038000 & -1.166101000 \\
\hline 6 & 2.623577000 & -0.339226000 & 0.076342000 & 6 & -0.833255000 & 0.306319000 & -0.256517000 & 6 & -0.801122000 & -0.328031000 \\
\hline 17 & 3.164763000 & -1.995606000 & 0.086258000 & 17 & -1.398393000 & 2.280034000 & -0.351516000 & 17 & 1.462357000 & 2.626135000 \\
\hline 6 & -3.729436000 & 0.558139000 & 0.073307000 & 6 & 1.899964000 & -0.206354000 & -0.084293000 & 6 & 1.572484000 & -1.029930000 \\
\hline 1 & -3.385121000 & 0.498787000 & 1.109237000 & 1 & 1.681689000 & -0.774163000 & 0.820260000 & 1 & 1.239473000 & -2.073659000 \\
\hline 1 & -4.209623000 & 1.534870000 & -0.031320000 & 1 & 1.701008000 & -0.864198000 & -0.931625000 & 1 & 1.718518000 & -0.770709000 \\
\hline 6 & -4.760425000 & -0.545006000 & -0.182812000 & 6 & 3.351138000 & 0.268421000 & -0.089740000 & 6 & 2.890395000 & -0.870989000 \\
\hline 1 & -4.271601000 & -1.518289000 & -0.077070000 & 1 & 3.514007000 & 0.946909000 & 0.751979000 & 1 & 2.735536000 & -1.118746000 \\
\hline 1 & -5.097220000 & -0.482130000 & -1.222448000 & 1 & 3.538734000 & 0.848581000 & -0.997623000 & 1 & 3.197699000 & 0.175955000 \\
\hline 6 & -5.963946000 & -0.466000000 & 0.755579000 & 6 & 4.335529000 & -0.898066000 & -0.009812000 & 6 & 3.992900000 & -1.757022000 \\
\hline 1 & -5.656616000 & -0.555184000 & 1.799607000 & 1 & 4.188913000 & -1.475814000 & 0.904116000 & 1 & 3.721141000 & -2.813278000 \\
\hline 1 & -6.487954000 & 0.485941000 & 0.647009000 & 1 & 4.214831000 & -1.576972000 & -0.855629000 & 1 & 4.193095000 & -1.508819000 \\
\hline 1 & -6.680729000 & -1.262457000 & 0.553110000 & 1 & 5.364751000 & -0.540205000 & -0.015247000 & 1 & 4.922898000 & -1.629894000 \\
\hline 7 & -2.546226000 & 0.546054000 & -0.790518000 & 7 & 0.921846000 & 0.887743000 & -0.153092000 & 7 & 0.544415000 & -0.140525000 \\
\hline 1 & -2.818108000 & 0.624615000 & -1.764032000 & 1 & 1.029980000 & 1.481520000 & -0.968853000 & 1 & 1.025706000 & 1.477662000 \\
\hline 1 & -2.047522000 & -0.332401000 & -0.700438000 & 1 & 0.924008000 & 1.487342000 & 0.666223000 & 1 & 0.630463000 & -0.052717000 \\
\hline 9 & 0.410765000 & -1.053741000 & -0.340058000 & 9 & -0.813481000 & 0.335518000 & 2.104413000 & 9 & -1.428858000 & 0.567139000 \\
\hline 1 & 1.372781000 & 3.313880000 & 0.044605000 & 1 & -2.527953000 & -3.209650000 & -0.111882000 & 1 & -4.572565000 & -0.618069000 \\
\hline & & & & & $n=$ & $R=$ & & & & \\
\hline 6 & -1.865581000 & 1.707836000 & -1.990248000 & 6 & -1.236733000 & 2.196762000 & -1.736944000 & 6 & -2.744719000 & 2.213425000 \\
\hline 6 & -1.529278000 & 0.374430000 & -1.809625000 & 6 & -1.427308000 & 0.831619000 & -1.652617000 & 6 & -2.783833000 & 0.850475000 \\
\hline 6 & -2.079435000 & 2.519147000 & -0.884865000 & 6 & -0.624443000 & 2.843382000 & -0.660976000 & 6 & -1.514809000 & 2.853312000 \\
\hline 1 & -1.360210000 & -0.261431000 & -2.664944000 & 1 & -1.912815000 & 0.305159000 & -2.461866000 & 1 & -3.731640000 & 0.335495000 \\
\hline 1 & -2.339264000 & 3.561090000 & -1.012872000 & 1 & -0.480079000 & 3.916274000 & -0.683092000 & 1 & -1.461669000 & 3.915447000 \\
\hline 6 & -1.403108000 & -0.166026000 & -0.531646000 & 6 & -1.017482000 & 0.086691000 & -0.541949000 & 6 & -1.615612000 & 0.125608000 \\
\hline 6 & -1.957819000 & 1.998533000 & 0.395423000 & 6 & -0.209148000 & 2.147904000 & 0.452865000 & 6 & -0.339706000 & 2.146030000 \\
\hline 1 & -2.114902000 & 2.620200000 & 1.263885000 & 1 & 0.214891000 & 2.674000000 & 1.297106000 & 1 & 0.604275000 & 2.666279000 \\
\hline 6 & -1.622810000 & 0.663867000 & 0.570917000 & 6 & -0.397073000 & 0.745422000 & 0.554272000 & 6 & -0.360659000 & 0.771174000 \\
\hline 17 & -1.486834000 & 0.071283000 & 2.209263000 & 17 & -1.094884000 & 0.249112000 & 2.370219000 & 17 & 1.814958000 & -0.729413000 \\
\hline 6 & 3.078313000 & 0.857903000 & -0.046555000 & 6 & 2.191645000 & 0.068595000 & -0.111135000 & 6 & 2.061285000 & 0.744624000 \\
\hline 1 & 2.618523000 & 0.416403000 & -0.934991000 & 1 & 1.772792000 & -0.462442000 & -0.965356000 & 1 & 1.850737000 & 1.419106000 \\
\hline 1 & 3.407451000 & 1.858464000 & -0.342225000 & 1 & 2.272149000 & 1.119879000 & -0.389552000 & 1 & 2.426605000 & 1.356161000 \\
\hline 6 & 4.298464000 & 0.024100000 & 0.357439000 & 6 & 3.554153000 & -0.504136000 & 0.269971000 & 6 & 3.135329000 & -0.262159000 \\
\hline 1 & 3.962593000 & -0.975730000 & 0.648686000 & 1 & 3.438150000 & -1.550075000 & 0.565905000 & 1 & 2.760262000 & -0.885040000 \\
\hline 1 & 4.751046000 & 0.468192000 & 1.249881000 & 1 & 3.940217000 & 0.026848000 & 1.144785000 & 1 & 3.327684000 & -0.929341000 \\
\hline 6 & 5.343662000 & -0.083648000 & -0.752171000 & 6 & 4.557170000 & -0.401622000 & -0.878711000 & 6 & 4.433710000 & 0.417671000 \\
\hline 1 & 4.924414000 & -0.552680000 & -1.644780000 & 1 & 4.211527000 & -0.952523000 & -1.754775000 & 1 & 4.276571000 & 1.072796000 \\
\hline 1 & 5.715548000 & 0.901447000 & -1.042229000 & 1 & 4.712010000 & 0.636406000 & -1.177527000 & 1 & 4.849081000 & 1.020746000 \\
\hline 1 & 6.200711000 & -0.680332000 & -0.437699000 & 1 & 5.523903000 & -0.812039000 & -0.588427000 & 1 & 5.184538000 & -0.320815000 \\
\hline 7 & 2.033024000 & 1.009646000 & 0.965426000 & 7 & 1.199643000 & -0.014213000 & 0.973068000 & 7 & 0.835742000 & 0.053404000 \\
\hline 1 & 2.412931000 & 1.424422000 & 1.808594000 & 1 & 1.486565000 & 0.480640000 & 1.812098000 & 1 & 1.318180000 & -0.494085000 \\
\hline 1 & 1.660338000 & 0.105054000 & 1.229795000 & 1 & 0.980472000 & -0.971161000 & 1.231948000 & 1 & 0.714305000 & -0.814576000 \\
\hline 1 & -1.957902000 & 2.107926000 & -2.990001000 & 1 & -1.565496000 & 2.747235000 & -2.605439000 & 1 & -3.661160000 & 2.762878000 \\
\hline 6 & -1.028752000 & -1.620364000 & -0.379546000 & 6 & -1.339390000 & -1.372965000 & -0.512338000 & 6 & -1.708387000 & -1.350924000 \\
\hline 9 & 0.143813000 & -1.778945000 & 0.276459000 & 9 & -0.333302000 & -2.122041000 & 0.052622000 & 9 & -1.094495000 & -1.698547000 \\
\hline 9 & -1.956468000 & -2.322134000 & 0.296764000 & 9 & -2.450197000 & -1.679866000 & 0.184037000 & 9 & -1.103527000 & -2.084133000 \\
\hline 9 & -0.880480000 & -2.224146000 & -1.575589000 & 9 & -1.515067000 & -1.880706000 & -1.750618000 & 9 & -2.972849000 & -1.787794000 \\
\hline & & & & & $n=2$ & & & & & \\
\hline 6 & 3.183459000 & $\begin{array}{l}-0.467837000 \\
\end{array}$ & 1.812601000 & 6 & -1.684173000 & 2.130846000 & -1.381992000 & 6 & -3.017455000 & 1.839917000 \\
\hline 6 & 2.339714000 & -1.218034000 & 1.014702000 & 6 & -1.675246000 & 0.768719000 & -1.563428000 & 6 & -2.949111000 & 0.526900000 \\
\hline 6 & 3.360566000 & 0.883023000 & 1.538417000 & 6 & -1.163089000 & 2.640798000 & -0.183096000 & 6 & -1.833329000 & 2.551972000 \\
\hline 1 & 2.192590000 & -2.271487000 & 1.201385000 & 1 & -2.074274000 & 0.323509000 & -2.464341000 & 1 & -3.847116000 & -0.057113000 \\
\hline 1 & 4.018231000 & 1.485927000 & 2.149890000 & 1 & -1.173227000 & 3.708228000 & 0.000106000 & 1 & -1.857925000 & 3.582695000 \\
\hline 6 & 1.641076000 & -0.650447000 & -0.058464000 & 6 & -1.152288000 & -0.121670000 & -0.606139000 & 6 & -1.727829000 & -0.104973000 \\
\hline 6 & 2.698372000 & 1.466599000 & 0.469681000 & 6 & -0.660108000 & 1.815377000 & 0.792103000 & 6 & -0.611084000 & 1.955472000 \\
\hline 1 & 2.841522000 & 2.511169000 & 0.236696000 & 1 & -0.325207000 & 2.224674000 & 1.735116000 & 1 & 0.291600000 & 2.528009000 \\
\hline 6 & 1.841742000 & 0.710812000 & -0.323386000 & 6 & -0.646184000 & 0.400872000 & 0.623987000 & 6 & -0.520139000 & 0.621044000 \\
\hline 17 & 1.102784000 & 1.549692000 & -1.670233000 & 17 & -1.271192000 & -0.480601000 & 2.255202000 & 17 & 1.566195000 & -1.211302000 \\
\hline 6 & -3.265338000 & 0.104809000 & 1.073852000 & 6 & 2.038086000 & 0.286257000 & -0.072218000 & 6 & 1.917974000 & 0.870946000 \\
\hline 1 & -3.137654000 & -0.980144000 & 1.016137000 & 1 & 1.667942000 & -0.010240000 & -1.054083000 & 1 & 1.689506000 & 1.622176000 \\
\hline 1 & -3.569002000 & 0.316214000 & 2.103386000 & 1 & 2.014513000 & 1.376185000 & -0.035534000 & 1 & 2.200583000 & 1.408680000 \\
\hline 6 & -4.387329000 & 0.532106000 & 0.120970000 & 6 & 3.454787000 & -0.237685000 & 0.152020000 & 6 & 3.086665000 & 0.002372000 \\
\hline 1 & -4.080671000 & 0.316502000 & -0.907390000 & 1 & 3.443006000 & -1.330663000 & 0.131226000 & 1 & 2.800742000 & -0.538249000 \\
\hline 1 & -4.508265000 & 1.618368000 & 0.181660000 & 1 & 3.795458000 & 0.051075000 & 1.150395000 & 1 & 3.289731000 & -0.749682000 \\
\hline 6 & -5.720249000 & -0.153335000 & 0.419007000 & 6 & 4.432488000 & 0.288095000 & -0.898398000 & 6 & 4.346067000 & 0.823266000 \\
\hline 1 & -5.633433000 & -1.238937000 & 0.336526000 & 1 & 4.134513000 & -0.016735000 & -1.902815000 & 1 & 4.176272000 & 1.573062000 \\
\hline 1 & -6.061914000 & 0.072066000 & 1.431469000 & 1 & 4.481906000 & 1.378124000 & -0.882597000 & 1 & 4.676508000 & 1.343324000 \\
\hline 1 & -6.500100000 & 0.168954000 & -0.272012000 & 1 & 5.437776000 & -0.092804000 & -0.720122000 & 1 & 5.164501000 & 0.184345000 \\
\hline 7 & -1.957447000 & 0.719770000 & 0.853994000 & 7 & 1.068419000 & -0.204870000 & 0.915305000 & 7 & 0.739066000 & 0.042051000 \\
\hline 1 & -2.025855000 & 1.729122000 & 0.912910000 & 1 & 1.285712000 & 0.074637000 & 1.866177000 & 1 & 1.163331000 & -0.727437000 \\
\hline 1 & -1.611623000 & 0.504172000 & -0.074076000 & 1 & 0.948801000 & -1.214937000 & 0.884419000 & 1 & 0.704789000 & -0.798539000 \\
\hline 1 & 3.700029000 & -0.932472000 & 2.640578000 & 1 & -2.089804000 & 2.789760000 & -2.135060000 & 1 & -3.973496000 & 2.302775000 \\
\hline 6 & 0.764518000 & -1.599199000 & -0.806214000 & 6 & -1.184649000 & -1.540999000 & -0.956558000 & 6 & -1.849333000 & -1.510576000 \\
\hline 8 & 0.918347000 & -2.796136000 & -0.789163000 & 8 & -1.788118000 & -2.038411000 & -1.879530000 & 8 & -2.883153000 & -2.127623000 \\
\hline 8 & -0.253302000 & -1.026300000 & -1.485176000 & 8 & -0.392315000 & -2.336666000 & -0.155668000 & 8 & -0.671331000 & -2.101091000 \\
\hline 1 & -0.728602000 & -1.754087000 & -1.913441000 & 1 & -0.530051000 & -3.239544000 & -0.474566000 & 1 & -0.896480000 & -3.004589000 \\
\hline
\end{tabular}




\begin{tabular}{|c|c|c|c|c|c|c|c|c|c|c|}
\hline & & & & & $\boldsymbol{n}=2$ & $\mathbf{R}=-\mathbf{C}$ & & & & \\
\hline 6 & 2.579721000 & 2.256063000 & 0.189628000 & 6 & 1.895832000 & 2.328859000 & 0.483991000 & 6 & -3.418241000 & -0.984947000 \\
\hline 6 & 1.838148000 & 1.504862000 & 1.085280000 & 6 & 1.679747000 & 1.274116000 & 1.345181000 & 6 & -3.155088000 & -0.011567000 \\
\hline 6 & 3.032926000 & 1.675246000 & -0.989721000 & 6 & 1.559271000 & 2.162633000 & -0.864991000 & 6 & -2.355285000 & -1.669714000 \\
\hline 1 & 1.475711000 & 1.941353000 & 2.004786000 & 1 & 1.922932000 & 1.360101000 & 2.395703000 & 1 & -3.960992000 & 0.531162000 \\
\hline 1 & 3.611606000 & 2.256268000 & -1.694694000 & 1 & 1.732581000 & 2.970279000 & -1.565068000 & 1 & -2.547744000 & -2.434216000 \\
\hline 6 & 1.539740000 & 0.164327000 & 0.813810000 & 6 & 1.140363000 & 0.058111000 & 0.898011000 & 6 & -1.838996000 & 0.283197000 \\
\hline 6 & 2.748503000 & 0.346328000 & -1.274500000 & 6 & 1.030304000 & 0.985075000 & -1.345869000 & 6 & -1.043482000 & -1.388987000 \\
\hline 1 & 3.096650000 & -0.111528000 & -2.188347000 & 1 & 0.834553000 & 0.865235000 & -2.402276000 & 1 & -0.237917000 & -1.929235000 \\
\hline 6 & 2.005743000 & -0.406045000 & -0.377154000 & 6 & 0.818614000 & -0.121358000 & -0.482514000 & 6 & -0.755316000 & -0.403555000 \\
\hline 17 & 1.660488000 & -2.070636000 & -0.747155000 & 17 & 1.470206000 & -1.806281000 & -1.274477000 & 17 & 1.508355000 & 1.974646000 \\
\hline 1 & 2.802185000 & 3.290526000 & 0.409525000 & 1 & 2.319520000 & 3.255703000 & 0.839873000 & 1 & -4.436892000 & -1.209570000 \\
\hline 6 & -3.027287000 & 0.484821000 & -0.400082000 & 6 & -1.891739000 & 0.279641000 & -0.154240000 & 6 & 1.646028000 & -1.030216000 \\
\hline 1 & -2.900013000 & 1.269269000 & 0.351259000 & 1 & -1.635473000 & 0.546933000 & 0.870695000 & 1 & 1.347400000 & -1.998527000 \\
\hline 1 & -2.979848000 & 0.986242000 & -1.371304000 & 1 & -1.754648000 & 1.170851000 & -0.767180000 & 1 & 1.852550000 & -1.182096000 \\
\hline 6 & -4.408813000 & -0.155753000 & -0.230262000 & 6 & -3.326292000 & -0.234756000 & -0.239413000 & 6 & 2.906390000 & -0.525714000 \\
\hline 1 & -4.451079000 & -0.653697000 & 0.743099000 & 1 & -3.420748000 & -1.141520000 & 0.363700000 & 1 & 2.690528000 & -0.360144000 \\
\hline 1 & -4.529743000 & -0.941798000 & -0.982404000 & 1 & -3.550564000 & -0.519923000 & -1.271172000 & 1 & 3.182233000 & 0.443137000 \\
\hline 6 & -5.552357000 & 0.851326000 & -0.346510000 & 6 & -4.335502000 & 0.808842000 & 0.238195000 & 6 & 4.071455000 & -1.503021000 \\
\hline 1 & -5.470122000 & 1.630617000 & 0.414140000 & 1 & -4.155490000 & 1.084709000 & 1.278254000 & 1 & 3.829244000 & -2.475953000 \\
\hline 1 & -5.549269000 & 1.342527000 & -1.322041000 & 1 & -4.280384000 & 1.717862000 & -0.362997000 & 1 & 4.333992000 & -1.657758000 \\
\hline 1 & -6.523214000 & 0.370252000 & -0.222296000 & 1 & -5.352688000 & 0.424874000 & 0.167769000 & 1 & 4.957280000 & -1.125758000 \\
\hline 7 & -1.878813000 & -0.415769000 & -0.302980000 & 7 & -0.893146000 & -0.707410000 & -0.600626000 & 7 & 0.555071000 & -0.054760000 \\
\hline 1 & -1.946265000 & -1.154273000 & -0.993829000 & 1 & -1.012188000 & -0.983421000 & -1.570949000 & 1 & 1.059275000 & 1.229882000 \\
\hline 1 & -1.852519000 & -0.869069000 & 0.603555000 & 1 & -0.906613000 & -1.555566000 & -0.037983000 & 1 & 0.614229000 & 0.473027000 \\
\hline 6 & 0.767692000 & -0.582660000 & 1.752238000 & 6 & 0.862236000 & -0.987953000 & 1.814377000 & 6 & -1.572704000 & 1.268052000 \\
\hline 7 & 0.142176000 & -1.153797000 & 2.533526000 & 7 & 0.560104000 & -1.836675000 & 2.539393000 & 7 & -1.302755000 & 2.044175000 \\
\hline & & & & & $n=$ & $\mathbf{R}=$ & & & & \\
\hline 6 & -3.758334000 & 1.615508000 & -0.037869000 & 6 & 2.091651000 & -2.222096000 & -0.000022000 & 6 & -3.675231000 & -0.423699000 \\
\hline 6 & -4.256249000 & 0.319417000 & -0.114197000 & 6 & 1.794192000 & -1.574391000 & -1.194919000 & 6 & -3.218231000 & 0.098034000 \\
\hline 6 & -2.387098000 & 1.826487000 & 0.054050000 & 6 & 1.794337000 & -1.574307000 & 1.194867000 & 6 & -2.752348000 & -0.860192000 \\
\hline 1 & -5.322132000 & 0.149006000 & -0.185763000 & 1 & 2.034538000 & -2.043642000 & -2.140823000 & 1 & -3.923236000 & 0.443623000 \\
\hline 1 & -1.995381000 & 2.833187000 & 0.113103000 & 1 & 2.034792000 & -2.043494000 & 2.140775000 & 1 & -3.091613000 & -1.266544000 \\
\hline 6 & -3.389744000 & -0.767745000 & -0.099046000 & 6 & 1.204082000 & -0.318125000 & -1.212515000 & 6 & -1.859681000 & 0.180641000 \\
\hline 6 & -1.503517000 & 0.752144000 & 0.070886000 & 6 & 1.204223000 & -0.318042000 & 1.212443000 & 6 & -1.386899000 & -0.783270000 \\
\hline 1 & -3.762444000 & -1.779771000 & -0.157467000 & 1 & 1.021926000 & 0.187989000 & -2.150339000 & 1 & -1.512339000 & 0.595856000 \\
\hline 1 & -0.433355000 & 0.899538000 & 0.140888000 & 1 & 1.022180000 & 0.188139000 & 2.150254000 & 1 & -0.688098000 & -1.123306000 \\
\hline 6 & -2.025742000 & -0.530400000 & -0.006306000 & 6 & 0.911939000 & 0.324212000 & -0.000041000 & 6 & -0.928040000 & -0.260728000 \\
\hline 17 & -0.934015000 & -1.912495000 & 0.012956000 & 17 & 1.467149000 & 2.331919000 & -0.000143000 & 17 & 1.365566000 & 2.681016000 \\
\hline 1 & -4.436894000 & 2.457571000 & -0.050200000 & 1 & 2.556370000 & -3.197280000 & -0.000016000 & 1 & -4.735309000 & -0.487211000 \\
\hline 6 & 3.377861000 & 0.738883000 & -0.003256000 & 6 & -1.825982000 & -0.182352000 & -0.000050000 & 6 & 1.421850000 & -1.006590000 \\
\hline 1 & 3.348296000 & 1.211605000 & -0.988782000 & 1 & -1.613567000 & -0.795842000 & -0.876452000 & 1 & 1.067563000 & -2.043479000 \\
\hline 1 & 3.549645000 & 1.546031000 & 0.714240000 & 1 & -1.613565000 & -0.795973000 & 0.876262000 & 1 & 1.505326000 & -0.720584000 \\
\hline 6 & 4.550099000 & -0.245627000 & 0.052057000 & 6 & -3.279635000 & 0.284768000 & -0.000019000 & 6 & 2.790142000 & -0.899320000 \\
\hline 1 & 4.373350000 & -1.049229000 & -0.669550000 & 1 & -3.459074000 & 0.913987000 & -0.876232000 & 1 & 2.698758000 & -1.172468000 \\
\hline 1 & 4.569645000 & -0.718849000 & 1.038839000 & 1 & -3.458870000 & 0.914457000 & 0.875900000 & 1 & 3.121098000 & 0.140594000 \\
\hline 6 & 5.898615000 & 0.415517000 & -0.230150000 & 6 & -4.258007000 & -0.889780000 & 0.000429000 & 6 & 3.829570000 & -1.795765000 \\
\hline 1 & 5.915383000 & 0.871215000 & -1.222412000 & 1 & -4.119820000 & -1.519088000 & -0.880247000 & 1 & 3.533777000 & -2.845925000 \\
\hline 1 & 6.112544000 & 1.202015000 & 0.496594000 & 1 & -4.120883000 & -1.517519000 & 0.882385000 & 1 & 3.967673000 & -1.524050000 \\
\hline 1 & 6.714510000 & -0.306640000 & -0.184880000 & 1 & -5.289577000 & -0.538499000 & -0.000514000 & 1 & 4.796841000 & -1.706578000 \\
\hline 7 & 2.051798000 & 0.179261000 & 0.262556000 & 7 & -0.848201000 & 0.913789000 & 0.000032000 & 7 & 0.453502000 & -0.111145000 \\
\hline 1 & 2.020168000 & -0.249490000 & 1.180586000 & 1 & -0.909672000 & 1.514051000 & 0.816611000 & 1 & 0.928438000 & 1.486362000 \\
\hline 1 & 1.826989000 & -0.553711000 & -0.400361000 & 1 & -0.909712000 & 1.514249000 & -0.816400000 & 1 & 0.615197000 & -0.070218000 \\
\hline & & & & & $\boldsymbol{n}=$ & D - & & & & \\
\hline 6 & -3.360852000 & 1.553610000 & 0.040040000 & 6 & 2.191922000 & -1.459636000 & 0.946373000 & 6 & 3.391028000 & 0.112518000 \\
\hline 6 & -1.964930000 & 1.530360000 & -0.017877000 & 6 & 1.973018000 & -1.237655000 & -0.415000000 & 6 & 2.662088000 & -0.644119000 \\
\hline 6 & -4.090853000 & 0.374562000 & 0.075063000 & 6 & 1.616936000 & -0.603876000 & 1.878597000 & 6 & 2.735396000 & 0.837263000 \\
\hline 1 & -5.171391000 & 0.407461000 & 0.120085000 & 1 & 1.798343000 & -0.758126000 & 2.935136000 & 1 & 3.307491000 & 1.423377000 \\
\hline 6 & -1.314550000 & 0.297624000 & -0.039973000 & 6 & 1.168035000 & -0.175958000 & -0.819129000 & 6 & 1.269064000 & -0.667551000 \\
\hline 6 & -3.445764000 & -0.857851000 & 0.051301000 & 6 & 0.817245000 & 0.462975000 & 1.494532000 & 6 & 1.354979000 & 0.815138000 \\
\hline 1 & -4.002728000 & -1.782699000 & 0.077208000 & 1 & 0.414769000 & 1.144340000 & 2.230610000 & 1 & 0.849746000 & 1.387279000 \\
\hline 6 & -2.062199000 & -0.871964000 & -0.005949000 & 6 & 0.598653000 & 0.685748000 & 0.128520000 & 6 & 0.607343000 & 0.056089000 \\
\hline 17 & -1.222410000 & -2.419172000 & -0.037745000 & 17 & 0.782481000 & 2.693272000 & -0.401359000 & 17 & -1.840372000 & 2.460260000 \\
\hline 6 & 3.488402000 & 0.437376000 & -0.005770000 & 6 & -1.988235000 & -0.358494000 & 0.033620000 & 6 & -1.591675000 & -1.037716000 \\
\hline 1 & 3.499898000 & 1.074840000 & -0.894024000 & 1 & -1.553424000 & -1.126719000 & -0.607073000 & 1 & -1.560443000 & -1.004422000 \\
\hline 1 & 3.460536000 & 1.112995000 & 0.853167000 & 1 & -1.797415000 & -0.659301000 & 1.064594000 & 1 & -1.148288000 & -1.990964000 \\
\hline 6 & 4.779013000 & -0.387896000 & 0.037759000 & 6 & -3.486635000 & -0.216928000 & -0.224382000 & 6 & -3.040358000 & -0.954263000 \\
\hline 1 & 4.791879000 & -1.069528000 & -0.818165000 & 1 & -3.648895000 & 0.102389000 & -1.257688000 & 1 & -3.460382000 & 0.005399000 \\
\hline 1 & 4.767171000 & -1.018222000 & 0.932455000 & 1 & -3.890707000 & 0.575501000 & 0.411815000 & 1 & -3.065681000 & -0.975386000 \\
\hline 6 & 6.038750000 & 0.476765000 & 0.032008000 & 6 & -4.239477000 & -1.521229000 & 0.036618000 & 6 & -3.892875000 & -2.095246000 \\
\hline 1 & 6.084882000 & 1.100663000 & -0.863067000 & 1 & -3.876640000 & -2.323199000 & -0.608414000 & 1 & -3.919237000 & -2.075752000 \\
\hline 1 & 6.064304000 & 1.141533000 & 0.897868000 & 1 & -4.118821000 & -1.846693000 & 1.071145000 & 1 & -3.504537000 & -3.068122000 \\
\hline 1 & 6.942602000 & -0.132802000 & 0.056687000 & 1 & -5.306074000 & -1.400566000 & -0.151568000 & 1 & -4.920213000 & -2.020906000 \\
\hline 7 & 2.236617000 & -0.321777000 & -0.014598000 & 7 & -1.225136000 & 0.873996000 & -0.199200000 & 7 & -0.807623000 & 0.091117000 \\
\hline 1 & 2.168951000 & -0.907452000 & 0.809826000 & 1 & -1.504063000 & 1.644859000 & 0.399607000 & 1 & -1.103537000 & 0.331325000 \\
\hline 1 & 2.192055000 & -0.942119000 & -0.814478000 & 1 & -1.272221000 & 1.207785000 & -1.156808000 & 1 & -1.354045000 & 1.456273000 \\
\hline 1 & -0.233392000 & 0.245627000 & -0.079954000 & 1 & 1.029034000 & 0.022678000 & -1.873768000 & 1 & 0.703913000 & -1.253740000 \\
\hline 1 & -3.878413000 & 2.504686000 & 0.060468000 & 1 & 2.815286000 & -2.282002000 & 1.269860000 & 1 & 4.471007000 & 0.136592000 \\
\hline 6 & -1.173982000 & 2.812721000 & -0.061423000 & 6 & 2.636924000 & -2.108756000 & -1.451107000 & 6 & 3.349273000 & -1.416184000 \\
\hline 1 & -1.419782000 & 3.394188000 & -0.953073000 & 1 & 3.645414000 & -1.749472000 & -1.671095000 & 1 & 3.192356000 & -0.940385000 \\
\hline 1 & -1.396290000 & 3.442405000 & 0.802349000 & 1 & 2.727751000 & -3.137650000 & -1.101855000 & 1 & 4.423485000 & -1.474569000 \\
\hline 1 & -0.103844000 & 2.614937000 & -0.074569000 & 1 & 2.081132000 & -2.114571000 & -2.388853000 & 1 & 2.962467000 & -2.433818000 \\
\hline & & & & & $n=3$ & & & & & \\
\hline 6 & 3.449774000 & -0.447458000 & 0.282772000 & 6 & 2.156174000 & -0.561030000 & 1.180345000 & 6 & 2.978911000 & 0.567991000 \\
\hline 6 & 2.163563000 & -0.896392000 & -0.015453000 & 6 & 1.933114000 & -0.515287000 & -0.196511000 & 6 & 2.377563000 & -0.372528000 \\
\hline 6 & 3.677503000 & 0.917645000 & 0.434556000 & 6 & 1.315882000 & 0.188977000 & 2.001819000 & 6 & 2.168623000 & 1.342184000 \\
\hline 1 & 4.675869000 & 1.264362000 & 0.665770000 & 1 & 1.484759000 & 0.183223000 & 3.071468000 & 1 & 2.627948000 & 2.075755000 \\
\hline 6 & 1.112672000 & 0.016272000 & -0.159539000 & 6 & 0.896425000 & 0.247413000 & -0.733736000 & 6 & 0.989013000 & -0.535294000 \\
\hline
\end{tabular}




\begin{tabular}{|c|c|c|c|c|c|c|c|c|}
\hline 6 & 2.652540000 & 1.839532000 & 0.295507000 & 6 & 0.278675000 & 0.956116000 & 1.503978000 & 6 \\
\hline 1 & 2.827286000 & 2.898069000 & 0.412435000 & 1 & -0.323840000 & 1.565986000 & 2.161328000 & 1 \\
\hline 6 & 1.380265000 & 1.361634000 & -0.001341000 & 6 & 0.074858000 & 0.995597000 & 0.111972000 & 6 \\
\hline 17 & 0.067981000 & 2.522088000 & -0.180044000 & 17 & -0.257606000 & 2.922681000 & -0.583715000 & 17 \\
\hline 6 & -3.417341000 & -0.748827000 & 0.060691000 & 6 & -2.102965000 & -0.728122000 & 0.046839000 & 6 \\
\hline 1 & -3.490625000 & -1.837527000 & -0.010682000 & 1 & -1.433071000 & -1.383956000 & -0.510676000 & 1 \\
\hline 1 & -3.087730000 & -0.533501000 & 1.080574000 & 1 & -1.892573000 & -0.883368000 & 1.105740000 & 1 \\
\hline 6 & -4.800810000 & -0.131211000 & -0.166181000 & 6 & -3.563371000 & -1.040362000 & -0.271921000 & 6 \\
\hline 1 & -5.123093000 & -0.348175000 & -1.189586000 & 1 & -3.750999000 & -0.856768000 & -1.333541000 & 1 \\
\hline 1 & -4.718681000 & 0.957713000 & -0.094915000 & 1 & -4.211245000 & -0.354185000 & 0.280752000 & 1 \\
\hline 6 & -5.849596000 & -0.636764000 & 0.823643000 & 6 & -3.927867000 & -2.483901000 & 0.073186000 & 6 \\
\hline 1 & -5.970046000 & -1.719868000 & 0.752558000 & 1 & -3.315608000 & -3.190806000 & -0.489103000 & 1 \\
\hline 1 & -5.565709000 & -0.404527000 & 1.852218000 & 1 & -3.778306000 & -2.686017000 & 1.135106000 & 1 \\
\hline 1 & -6.824206000 & -0.183273000 & 0.639699000 & 1 & -4.972277000 & -2.689263000 & -0.160472000 & 1 \\
\hline 7 & -2.358576000 & -0.310819000 & -0.850052000 & 7 & -1.705617000 & 0.649879000 & -0.267734000 & 7 \\
\hline 1 & -2.221536000 & 0.691770000 & -0.787216000 & 1 & -2.217308000 & 1.351501000 & 0.258159000 & 1 \\
\hline 1 & -2.606892000 & -0.512937000 & -1.811691000 & 1 & -1.799291000 & 0.885248000 & -1.250910000 & 1 \\
\hline 1 & 0.116497000 & -0.341429000 & -0.387739000 & 1 & 0.791435000 & 0.297079000 & -1.807752000 & 1 \\
\hline 1 & 4.269076000 & -1.139629000 & 0.397911000 & 1 & 2.957037000 & -1.141130000 & 1.608209000 & 1 \\
\hline 8 & 1.825877000 & -2.202649000 & -0.185818000 & 8 & 2.685404000 & -1.186055000 & -1.117020000 & 8 \\
\hline 6 & 2.835158000 & -3.189232000 & -0.049233000 & 6 & 3.784674000 & -1.954011000 & -0.658516000 & 6 \\
\hline 1 & 3.630772000 & -3.054417000 & -0.786819000 & 1 & 4.518240000 & -1.333102000 & -0.138114000 & 1 \\
\hline 1 & 3.268999000 & -3.182862000 & 0.954195000 & 1 & 3.462389000 & -2.760878000 & 0.005193000 & 1 \\
\hline 1 & 2.344972000 & -4.143540000 & -0.221583000 & 1 & 4.242468000 & -2.382194000 & -1.546665000 & 1 \\
\hline & & & & & $n=$ & 3, $\mathrm{R}=$ & & \\
\hline 6 & 3.118922000 & -1.740354000 & 0.069317000 & 6 & 2.166208000 & -1.598614000 & 0.822623000 & 6 \\
\hline 6 & 1.757902000 & -1.529473000 & -0.067645000 & 6 & 1.894717000 & -1.278203000 & -0.496171000 & 6 \\
\hline 6 & 3.946551000 & -0.630487000 & 0.188531000 & 6 & 1.644518000 & -0.745368000 & 1.791644000 & 6 \\
\hline 9 & 0.945322000 & -2.599543000 & -0.184146000 & 9 & 2.395463000 & -2.066486000 & -1.474577000 & 9 \\
\hline 1 & 5.013699000 & -0.767899000 & 0.296580000 & 1 & 1.847926000 & -0.941602000 & 2.836594000 & 1 \\
\hline 6 & 1.188710000 & -0.267488000 & -0.090871000 & 6 & 1.142203000 & -0.186550000 & -0.883849000 & 6 \\
\hline 6 & 3.420879000 & 0.655960000 & 0.170272000 & 6 & 0.881116000 & 0.364274000 & 1.466019000 & 6 \\
\hline 1 & 4.060894000 & 1.520395000 & 0.262421000 & 1 & 0.532310000 & 1.034362000 & 2.238651000 & 1 \\
\hline 6 & 2.048404000 & 0.815291000 & 0.030639000 & 6 & 0.629793000 & 0.662114000 & 0.112408000 & 6 \\
\hline 17 & 1.378064000 & 2.437718000 & 0.006970000 & 17 & 0.895355000 & 2.651704000 & -0.328339000 & 17 \\
\hline 6 & -3.329908000 & -0.309872000 & 0.023708000 & 6 & -1.965743000 & -0.307249000 & 0.041936000 & 6 \\
\hline 1 & -3.319620000 & -1.224816000 & -0.574276000 & 1 & -1.568830000 & -1.054888000 & -0.645549000 & 1 \\
\hline 1 & -3.151080000 & -0.620960000 & 1.056261000 & 1 & -1.760503000 & -0.661194000 & 1.052824000 & 1 \\
\hline 6 & -4.707291000 & 0.351374000 & -0.078254000 & 6 & -3.463096000 & -0.104498000 & -0.175065000 & 6 \\
\hline 1 & -4.875259000 & 0.664461000 & -1.113564000 & 1 & -3.635965000 & 0.270810000 & -1.187585000 & 1 \\
\hline 1 & -4.707363000 & 1.266310000 & 0.522454000 & 1 & -3.828259000 & 0.667049000 & 0.508556000 & 1 \\
\hline 6 & -5.843671000 & -0.564285000 & 0.374876000 & 6 & -4.252412000 & -1.396108000 & 0.036681000 & 6 \\
\hline 1 & -5.885028000 & -1.471718000 & -0.231076000 & 1 & -3.928375000 & -2.175377000 & -0.654946000 & 1 \\
\hline 1 & -5.714070000 & -0.869193000 & 1.415347000 & 1 & -4.123052000 & -1.776841000 & 1.051073000 & 1 \\
\hline 1 & -6.811564000 & -0.068550000 & 0.294038000 & 1 & -5.317677000 & -1.231853000 & -0.123307000 & 1 \\
\hline 7 & -2.191004000 & 0.510281000 & -0.397853000 & 7 & -1.170335000 & 0.914461000 & -0.150893000 & 7 \\
\hline 1 & -2.137778000 & 1.359864000 & 0.152986000 & 1 & -1.416434000 & 1.661134000 & 0.492152000 & 1 \\
\hline 1 & -2.299358000 & 0.800725000 & -1.363159000 & 1 & -1.238756000 & 1.295832000 & -1.089873000 & 1 \\
\hline 1 & 0.117094000 & -0.142129000 & -0.198508000 & 1 & 1.017958000 & 0.031597000 & -1.934156000 & 1 \\
\hline 1 & 3.508475000 & -2.747768000 & 0.080859000 & 1 & 2.767983000 & -2.459633000 & 1.067433000 & 1 \\
\hline & & & & & $n-$ & D - & & \\
\hline 6 & 1.306296000 & -0.750853000 & 1.635391000 & 6 & 1.594083000 & 0.055107000 & 1.687850000 & 6 \\
\hline 6 & 1.298626000 & -0.881067000 & 0.250738000 & 6 & 1.553745000 & -0.122307000 & 0.309090000 & 6 \\
\hline 6 & 1.562127000 & 0.489623000 & 2.202681000 & 6 & 0.578552000 & 0.801305000 & 2.278797000 & 6 \\
\hline 1 & 1.572591000 & 0.598289000 & 3.278355000 & 1 & 0.591726000 & 0.972009000 & 3.347491000 & 1 \\
\hline 6 & 1.542772000 & 0.216640000 & -0.566455000 & 6 & 0.538177000 & 0.417583000 & -0.470021000 & 6 \\
\hline 6 & 1.805326000 & 1.596887000 & 1.400589000 & 6 & -0.452291000 & 1.348369000 & 1.534429000 & 6 \\
\hline 1 & 1.540648000 & 0.116984000 & -1.640663000 & 1 & 0.554461000 & 0.296547000 & -1.543329000 & 1 \\
\hline 1 & 2.000645000 & 2.565629000 & 1.835792000 & 1 & -1.205652000 & 1.959392000 & 2.011910000 & 1 \\
\hline 6 & 1.791664000 & 1.446121000 & 0.021882000 & 6 & -0.481813000 & 1.167999000 & 0.137853000 & 6 \\
\hline 17 & 2.094522000 & 2.834784000 & -0.999276000 & 17 & -0.912950000 & 2.898515000 & -0.871933000 & 17 \\
\hline 1 & 1.120674000 & -1.611297000 & 2.261032000 & 1 & 2.391358000 & -0.368757000 & 2.276922000 & 1 \\
\hline 6 & -2.997101000 & 0.607762000 & 0.300365000 & 6 & -2.379131000 & -0.846019000 & 0.079093000 & 6 \\
\hline 1 & -2.606705000 & -0.397016000 & 0.119872000 & 1 & -1.570818000 & -1.468474000 & -0.305924000 & 1 \\
\hline 1 & -3.089571000 & 0.706540000 & 1.385634000 & 1 & -2.287941000 & -0.838671000 & 1.165809000 & 1 \\
\hline 6 & -4.383299000 & 0.737286000 & -0.338940000 & 6 & -3.739677000 & -1.386404000 & -0.353195000 & 6 \\
\hline 1 & -4.283974000 & 0.634316000 & -1.424128000 & 1 & -3.811970000 & -1.354867000 & -1.443891000 & 1 \\
\hline 1 & -4.763840000 & 1.748057000 & -0.160384000 & 1 & -4.528667000 & -0.732832000 & 0.029318000 & 1 \\
\hline 6 & -5.382424000 & -0.291922000 & 0.188131000 & 6 & -3.970673000 & -2.815299000 & 0.137533000 & 6 \\
\hline 1 & -5.037542000 & -1.310093000 & -0.003302000 & 1 & -3.212867000 & -3.495186000 & -0.254708000 & 1 \\
\hline 1 & -5.522318000 & -0.189618000 & 1.266401000 & 1 & -3.935701000 & -2.870428000 & 1.226751000 & 1 \\
\hline 1 & -6.359190000 & -0.179499000 & -0.283688000 & 1 & -4.945103000 & -3.183239000 & -0.182385000 & 1 \\
\hline 7 & -1.985155000 & 1.565904000 & -0.149182000 & 7 & -2.116853000 & 0.526788000 & -0.382142000 & 7 \\
\hline 1 & -2.291998000 & 2.515554000 & 0.028665000 & 1 & -2.783887000 & 1.204894000 & -0.025364000 & 1 \\
\hline 1 & -1.849876000 & 1.492409000 & -1.151092000 & 1 & -2.115129000 & 0.616698000 & -1.394168000 & 1 \\
\hline 6 & 0.966055000 & -2.208248000 & -0.375317000 & 6 & 2.657644000 & -0.868675000 & -0.390630000 & 6 \\
\hline 9 & 1.371542000 & -3.244505000 & 0.384475000 & 9 & 3.346805000 & -1.671020000 & 0.444196000 & 9 \\
\hline 9 & -0.369244000 & -2.356241000 & -0.549834000 & 9 & 2.187306000 & -1.653591000 & -1.389260000 & 9 \\
\hline 9 & 1.530972000 & -2.356618000 & -1.589632000 & 9 & 3.555512000 & -0.031978000 & -0.956643000 & 9 \\
\hline & & & & & $n=3$ & $--C$ & OH & \\
\hline 6 & 2.347101000 & -1.623571000 & 0.139585000 & 6 & 1.916148000 & -0.160308000 & 1.589494000 & 6 \\
\hline 6 & 2.954254000 & -0.380513000 & -0.039760000 & 6 & 1.825214000 & -0.326170000 & 0.206797000 & 6 \\
\hline 6 & 0.973684000 & -1.702323000 & 0.310336000 & 6 & 0.970100000 & 0.632928000 & 2.223521000 & 6 \\
\hline 1 & 0.499746000 & -2.664416000 & 0.449468000 & 1 & 1.029550000 & 0.793769000 & 3.292264000 & 1 \\
\hline 6 & 2.181289000 & 0.781585000 & -0.047354000 & 6 & 0.808629000 & 0.281435000 & -0.528562000 & 6 \\
\hline 6 & 0.188884000 & -0.552958000 & 0.305365000 & 6 & -0.054280000 & 1.247639000 & 1.517393000 & 6 \\
\hline 1 & -0.884934000 & -0.609003000 & 0.439430000 & 1 & -0.753517000 & 1.897303000 & 2.025386000 & 1 \\
\hline 6 & 0.810919000 & 0.675622000 & 0.125752000 & 6 & -0.137675000 & 1.083405000 & 0.124692000 & 6 \\
\hline 17 & -0.160688000 & 2.140014000 & 0.116565000 & 17 & -0.497452000 & 2.863852000 & -0.861690000 & 17 \\
\hline 6 & -4.362746000 & -0.671082000 & -0.114135000 & 6 & -2.202849000 & -0.782529000 & 0.106315000 & 6 \\
\hline
\end{tabular}




\begin{tabular}{|c|c|c|c|c|c|c|c|c|}
\hline 1 & -4.027403000 & -0.514247000 & -1.142618000 & 1 & -1.449656000 & -1.465611000 & -0.288168000 & 1 \\
\hline 1 & -4.486786000 & -1.750950000 & 0.003724000 & 1 & -2.097756000 & -0.783433000 & 1.191871000 & 1 \\
\hline 6 & -5.712962000 & 0.021277000 & 0.092138000 & 6 & -3.605763000 & -1.219045000 & -0.307792000 & 6 \\
\hline 1 & -5.579794000 & 1.101092000 & -0.026051000 & 1 & -3.688592000 & -1.184163000 & -1.397622000 & 1 \\
\hline 1 & -6.038610000 & -0.136455000 & 1.125187000 & 1 & -4.337712000 & -0.506199000 & 0.082032000 & 1 \\
\hline 6 & -6.790932000 & -0.475037000 & -0.870541000 & 6 & -3.939299000 & -2.625242000 & 0.189953000 & 6 \\
\hline 1 & -6.502923000 & -0.301439000 & -1.909398000 & 1 & -3.240663000 & -3.362139000 & -0.209458000 & 1 \\
\hline 1 & -6.963763000 & -1.546680000 & -0.751640000 & 1 & -3.894575000 & -2.680630000 & 1.278811000 & 1 \\
\hline 1 & -7.740703000 & 0.033401000 & -0.701896000 & 1 & -4.942949000 & -2.918767000 & -0.116438000 & 1 \\
\hline 7 & -3.279372000 & -0.246167000 & 0.776719000 & 7 & -1.843252000 & 0.565213000 & -0.359104000 & 7 \\
\hline 1 & -3.539799000 & -0.390271000 & 1.745952000 & 1 & -2.444262000 & 1.295150000 & 0.011706000 & 1 \\
\hline 1 & -3.099542000 & 0.746326000 & 0.671203000 & 1 & -1.852741000 & 0.658548000 & -1.370464000 & 1 \\
\hline 1 & 2.643485000 & 1.745982000 & -0.185981000 & 1 & 0.783415000 & 0.174637000 & -1.601829000 & 1 \\
\hline 1 & 2.9656660000 & -2.509044000 & 0.141917000 & 1 & 2.717696000 & -0.636442000 & 2.131787000 & 1 \\
\hline 6 & 4.428539000 & -0.338627000 & -0.220018000 & 6 & 2.853157000 & -1.164567000 & -0.463226000 & 6 \\
\hline 8 & 5.150559000 & -1.305516000 & -0.222571000 & 8 & 3.770001000 & -1.717608000 & 0.094599000 & 8 \\
\hline 8 & 4.908005000 & 0.918076000 & -0.386497000 & 8 & 2.671561000 & -1.270849000 & -1.805829000 & 8 \\
\hline 1 & 5.865967000 & 0.831909000 & -0.493601000 & 1 & 3.398423000 & -1.821627000 & -2.129092000 & 1 \\
\hline & & & & & $\boldsymbol{n}=3$ & $\mathbf{R}=-\mathbf{r}$ & & \\
\hline 6 & -2.588698000 & 1.720036000 & -0.113184000 & 6 & 2.155795000 & -0.811957000 & 1.367334000 & 6 \\
\hline 6 & -3.374349000 & 0.573402000 & 0.038076000 & 6 & 1.986354000 & -0.839328000 & -0.019946000 & 6 \\
\hline 6 & -1.211772000 & 1.595050000 & -0.216129000 & 6 & 1.396130000 & 0.094523000 & 2.096881000 & 6 \\
\hline 1 & -0.599470000 & 2.478566000 & -0.332800000 & 1 & 1.520369000 & 0.154395000 & 3.170264000 & 1 \\
\hline 6 & -2.776577000 & -0.689908000 & 0.084872000 & 6 & 1.081695000 & 0.006101000 & -0.663907000 & 6 \\
\hline 6 & -0.600922000 & 0.346181000 & -0.171207000 & 6 & 0.487181000 & 0.943853000 & 1.488288000 & 6 \\
\hline 1 & 0.475955000 & 0.250795000 & -0.250844000 & 1 & -0.056093000 & 1.670083000 & 2.076847000 & 1 \\
\hline 6 & -1.398664000 & -0.781239000 & -0.020824000 & 6 & 0.320181000 & 0.917617000 & 0.088221000 & 6 \\
\hline 17 & -0.650611000 & -2.365007000 & 0.037928000 & 17 & 0.280734000 & 2.784480000 & -0.732997000 & 17 \\
\hline 6 & 3.980149000 & 0.615285000 & 0.026219000 & 6 & -2.026879000 & -0.545494000 & 0.064974000 & 6 \\
\hline 1 & 3.977810000 & 1.530734000 & -0.571349000 & 1 & -1.428208000 & -1.321232000 & -0.413438000 & 1 \\
\hline 1 & 3.782974000 & 0.923988000 & 1.056156000 & 1 & -1.877867000 & -0.642426000 & 1.140803000 & 1 \\
\hline 6 & 5.361045000 & -0.041025000 & -0.053417000 & 6 & -3.502231000 & -0.689152000 & -0.299702000 & 6 \\
\hline 1 & 5.547440000 & -0.352205000 & -1.086077000 & 1 & -3.623376000 & -0.560163000 & -1.378756000 & 1 \\
\hline 1 & 5.354264000 & -0.956453000 & 0.546312000 & 1 & -4.073718000 & 0.113009000 & 0.175533000 & 1 \\
\hline 6 & 6.486166000 & 0.878338000 & 0.419779000 & 6 & -4.066027000 & -2.045310000 & 0.123423000 & 6 \\
\hline 1 & 6.534739000 & 1.786597000 & -0.184402000 & 1 & -3.533899000 & -2.864004000 & -0.363361000 & 1 \\
\hline 1 & 6.338533000 & 1.181124000 & 1.458437000 & 1 & -3.983470000 & -2.188950000 & 1.201934000 & 1 \\
\hline 1 & 7.456757000 & 0.385914000 & 0.354268000 & 1 & -5.119138000 & -2.128218000 & -0.143262000 & 1 \\
\hline 7 & 2.850678000 & -0.208995000 & -0.415970000 & 7 & -1.446916000 & 0.748110000 & -0.332950000 & 7 \\
\hline 1 & 2.796630000 & -1.060269000 & 0.132674000 & 1 & -1.895150000 & 1.542927000 & 0.114069000 & 1 \\
\hline 1 & 2.981078000 & -0.499765000 & -1.378707000 & 1 & -1.486801000 & 0.911586000 & -1.335096000 & 1 \\
\hline 1 & -3.380310000 & -1.576904000 & 0.201529000 & 1 & 1.009780000 & -0.006224000 & -1.741500000 & 1 \\
\hline 1 & -3.061125000 & 2.690830000 & -0.148325000 & 1 & 2.865491000 & -1.468658000 & 1.845380000 & 1 \\
\hline 6 & -4.795465000 & 0.686667000 & 0.145950000 & 6 & 2.753145000 & -1.751480000 & -0.812447000 & 6 \\
\hline 7 & -5.940730000 & 0.781201000 & 0.232761000 & 7 & 3.360849000 & -2.494469000 & -1.450935000 & 7 \\
\hline & & & & & $\boldsymbol{n}=$ & 11 & & \\
\hline 6 & -3.758334000 & 1.615508000 & -0.037869000 & 6 & 2.091651000 & -2.222096000 & -0.000022000 & 6 \\
\hline 6 & -4.256249000 & 0.319417000 & -0.114197000 & 6 & 1.794192000 & -1.574391000 & -1.194919000 & 6 \\
\hline 6 & -2.387098000 & 1.826487000 & 0.054050000 & 6 & 1.794337000 & -1.574307000 & 1.194867000 & 6 \\
\hline 1 & -5.322132000 & 0.149006000 & -0.185763000 & 1 & 2.034538000 & -2.043642000 & -2.140823000 & 1 \\
\hline 1 & -1.995381000 & 2.833187000 & 0.113103000 & 1 & 2.034792000 & -2.043494000 & 2.140775000 & 1 \\
\hline 6 & -3.389744000 & -0.767745000 & -0.099046000 & 6 & 1.204082000 & -0.318125000 & -1.212515000 & 6 \\
\hline 6 & -1.503517000 & 0.752144000 & 0.070886000 & 6 & 1.204223000 & -0.318042000 & 1.212443000 & 6 \\
\hline 1 & -3.762444000 & -1.779771000 & -0.157467000 & 1 & 1.021926000 & 0.187989000 & -2.150339000 & 1 \\
\hline 1 & -0.433355000 & 0.899538000 & 0.140888000 & 1 & 1.022180000 & 0.188139000 & 2.150254000 & 1 \\
\hline 6 & -2.025742000 & -0.530400000 & -0.006306000 & 6 & 0.911939000 & 0.324212000 & -0.000041000 & 6 \\
\hline 17 & -0.934015000 & -1.912495000 & 0.012956000 & 17 & 1.467149000 & 2.331919000 & -0.000143000 & 17 \\
\hline 1 & -4.436894000 & 2.457571000 & -0.050200000 & 1 & 2.556370000 & -3.197280000 & -0.000016000 & 1 \\
\hline 6 & 3.377861000 & 0.738883000 & -0.003256000 & 6 & -1.825982000 & -0.182352000 & -0.000050000 & 6 \\
\hline 1 & 3.348296000 & 1.211605000 & -0.988782000 & 1 & -1.613567000 & -0.795842000 & -0.876452000 & 1 \\
\hline 1 & 3.549645000 & 1.546031000 & 0.714240000 & 1 & -1.613565000 & -0.795973000 & 0.876262000 & 1 \\
\hline 6 & 4.550099000 & -0.245627000 & 0.052057000 & 6 & -3.279635000 & 0.284768000 & -0.000019000 & 6 \\
\hline 1 & 4.373350000 & -1.049229000 & -0.669550000 & 1 & -3.459074000 & 0.913987000 & -0.876232000 & 1 \\
\hline 1 & 4.569645000 & -0.718849000 & 1.038839000 & 1 & -3.458870000 & 0.914457000 & 0.875900000 & 1 \\
\hline 6 & 5.898615000 & 0.415517000 & -0.230150000 & 6 & -4.258007000 & -0.889780000 & 0.000429000 & 6 \\
\hline 1 & 5.915383000 & 0.871215000 & -1.222412000 & 1 & -4.119820000 & -1.519088000 & -0.880247000 & 1 \\
\hline 1 & 6.112544000 & 1.202015000 & 0.496594000 & 1 & -4.120883000 & -1.517519000 & 0.882385000 & 1 \\
\hline 1 & 6.714510000 & -0.306640000 & -0.184880000 & 1 & -5.289577000 & -0.538499000 & -0.000514000 & 1 \\
\hline 7 & 2.051798000 & 0.179261000 & 0.262556000 & 7 & -0.848201000 & 0.913789000 & 0.000032000 & 7 \\
\hline 1 & 2.020168000 & -0.249490000 & 1.180586000 & 1 & -0.909672000 & 1.514051000 & 0.816611000 & 1 \\
\hline 1 & 1.826989000 & -0.553711000 & -0.400361000 & 1 & -0.909712000 & 1.514249000 & -0.816400000 & 1 \\
\hline & & & & & $\boldsymbol{n}=$ & $\mathbf{R}=-$ & & \\
\hline 6 & 3.302257000 & 1.248237000 & -0.010438000 & 6 & -2.443679000 & -1.171571000 & 0.000974000 & 6 \\
\hline 6 & 3.853512000 & -0.033560000 & -0.015195000 & 6 & -1.923785000 & -0.657392000 & 1.188245000 & 6 \\
\hline 6 & 1.912193000 & 1.361831000 & -0.003526000 & 6 & -1.925552000 & -0.655187000 & -1.187301000 & 6 \\
\hline 1 & 4.929755000 & -0.153799000 & -0.027209000 & 1 & -2.312300000 & -1.008727000 & 2.137260000 & 1 \\
\hline 1 & 1.457141000 & 2.344614000 & -0.006561000 & 1 & -2.315686000 & -1.004771000 & -2.136424000 & 1 \\
\hline 6 & 3.049782000 & -1.166401000 & -0.007772000 & 6 & -0.929495000 & 0.312689000 & 1.208604000 & 6 \\
\hline 6 & 1.086416000 & 0.243240000 & 0.003969000 & 6 & -0.931962000 & 0.314216000 & -1.207512000 & 6 \\
\hline 1 & 0.008647000 & 0.347753000 & 0.006429000 & 1 & -0.590896000 & 0.724632000 & -2.147764000 & 1 \\
\hline 6 & 1.671188000 & -1.013963000 & 0.003009000 & 6 & -0.432963000 & 0.814788000 & 0.000862000 & 6 \\
\hline 17 & 0.649365000 & -2.446961000 & 0.008406000 & 17 & -0.257154000 & 2.902560000 & 0.000626000 & 17 \\
\hline 6 & -3.678577000 & 0.787604000 & 0.003867000 & 6 & 1.985600000 & -0.588158000 & -0.002297000 & 6 \\
\hline 1 & -3.641511000 & 1.433521000 & 0.885293000 & 1 & 1.578039000 & -1.096108000 & 0.872752000 & 1 \\
\hline 1 & -3.639206000 & 1.454326000 & -0.861830000 & 1 & 1.581306000 & -1.094237000 & -0.879914000 & 1 \\
\hline 6 & -5.004116000 & 0.020038000 & -0.007123000 & 6 & 3.511712000 & -0.639305000 & 0.000495000 & 6 \\
\hline 1 & -5.035822000 & -0.645982000 & 0.860795000 & 1 & 3.890571000 & -0.107464000 & 0.877675000 & 1 \\
\hline 1 & -5.032797000 & -0.625928000 & -0.890184000 & 1 & 3.893974000 & -0.105761000 & -0.874161000 & 1 \\
\hline 6 & -6.224892000 & 0.939166000 & 0.001264000 & 6 & 4.037971000 & -2.074403000 & 0.000149000 & 6 \\
\hline 1 & -6.234713000 & 1.574347000 & 0.889497000 & 1 & 3.695418000 & -2.621292000 & 0.880200000 & 1 \\
\hline
\end{tabular}




\begin{tabular}{|c|c|c|c|c|c|c|c|c|}
\hline 1 & -6.231210000 & 1.594933000 & -0.871908000 & 1 & 3.698866000 & -2.619622000 & -0.882274000 & 1 \\
\hline 1 & -7.154320000 & 0.368877000 & -0.007246000 & 1 & 5.127606000 & -2.090767000 & 0.002257000 & 1 \\
\hline 7 & -2.461545000 & -0.026338000 & -0.004264000 & 7 & 1.434709000 & 0.772561000 & -0.001640000 & 7 \\
\hline 1 & -2.434691000 & -0.624388000 & -0.822422000 & 1 & 1.692301000 & 1.318310000 & -0.817891000 & 1 \\
\hline 1 & -2.436366000 & -0.643458000 & 0.799658000 & 1 & 1.693361000 & 1.317661000 & 0.814713000 & 1 \\
\hline 1 & 3.486769000 & -2.154457000 & -0.014039000 & 1 & -0.587432000 & 0.721550000 & 2.149152000 & 1 \\
\hline 6 & 4.183001000 & 2.470193000 & 0.011518000 & 6 & -3.506134000 & -2.240463000 & -0.000788000 & 6 \\
\hline 1 & 4.460492000 & 2.736788000 & 1.034751000 & 1 & -3.078521000 & -3.242343000 & -0.104682000 & 1 \\
\hline 1 & 5.107493000 & 2.305153000 & -0.542167000 & 1 & -4.080203000 & -2.229507000 & 0.926349000 & 1 \\
\hline 1 & 3.677374000 & 3.332098000 & -0.423340000 & 1 & -4.208040000 & -2.104273000 & -0.824998000 & 1 \\
\hline & & & & & $n=4$ & $\mathbf{R}$ & Me & \\
\hline 6 & -3.152968000 & 0.626611000 & 0.000000000 & 6 & -2.385910000 & -0.216479000 & -0.000007000 & 6 \\
\hline 6 & -1.796935000 & 0.948291000 & -0.000040000 & 6 & -1.761261000 & 0.113391000 & 1.197447000 & 6 \\
\hline 6 & -3.542173000 & -0.715443000 & 0.000005000 & 6 & -1.761266000 & 0.113411000 & -1.197457000 & 6 \\
\hline 1 & -1.467391000 & 1.976001000 & -0.000043000 & 1 & -2.259576000 & -0.106154000 & 2.132820000 & 1 \\
\hline 1 & -4.596997000 & -0.951593000 & 0.000038000 & 1 & -2.259585000 & -0.106121000 & -2.132831000 & 1 \\
\hline 6 & -0.836434000 & -0.061367000 & -0.000079000 & 6 & -0.527853000 & 0.752336000 & 1.210615000 & 6 \\
\hline 6 & -2.590670000 & -1.719746000 & -0.000033000 & 6 & -0.527859000 & 0.752358000 & -1.210619000 & 6 \\
\hline 1 & -2.891615000 & -2.757293000 & -0.000031000 & 1 & -0.081474000 & 1.049726000 & -2.149135000 & 1 \\
\hline 6 & -1.241218000 & -1.384163000 & -0.000077000 & 6 & 0.092829000 & 1.085137000 & -0.000001000 & 6 \\
\hline 17 & -0.036550000 & -2.667933000 & -0.000122000 & 17 & 0.865256000 & 3.033529000 & 0.000010000 & 17 \\
\hline 6 & 3.877442000 & 0.953221000 & -0.000343000 & 6 & 2.004609000 & -0.954238000 & 0.000002000 & 6 \\
\hline 1 & 3.803378000 & 1.605474000 & -0.874797000 & 1 & 1.469755000 & -1.322218000 & 0.876670000 & 1 \\
\hline 1 & 3.803245000 & 1.607419000 & 0.872646000 & 1 & 1.469748000 & -1.322219000 & -0.876662000 & 1 \\
\hline 6 & 5.242832000 & 0.259070000 & 0.000541000 & 6 & 3.450932000 & -1.445285000 & -0.000004000 & 6 \\
\hline 1 & 5.308487000 & -0.395414000 & -0.874205000 & 1 & 3.969409000 & -1.045687000 & 0.875959000 & 1 \\
\hline 1 & 5.308336000 & -0.393467000 & 0.876753000 & 1 & 3.969402000 & -1.045684000 & -0.875971000 & 1 \\
\hline 6 & 6.412566000 & 1.242195000 & -0.000441000 & 6 & 3.538301000 & -2.971096000 & -0.000007000 & 6 \\
\hline 1 & 6.386635000 & 1.886344000 & -0.881924000 & 1 & 3.053841000 & -3.394623000 & 0.881319000 & 1 \\
\hline 1 & 6.386429000 & 1.888339000 & 0.879575000 & 1 & 3.053840000 & -3.394620000 & -0.881334000 & 1 \\
\hline 1 & 7.371088000 & 0.722221000 & 0.000260000 & 1 & 4.576382000 & -3.302535000 & -0.000009000 & 1 \\
\hline 7 & 2.707234000 & 0.073079000 & 0.000538000 & 7 & 1.869438000 & 0.507237000 & 0.000002000 & 7 \\
\hline 1 & 2.716063000 & -0.535105000 & 0.811699000 & 1 & 2.273397000 & 0.955695000 & -0.816212000 & 1 \\
\hline 1 & 2.716096000 & -0.536802000 & -0.809348000 & 1 & 2.273396000 & 0.955694000 & 0.816216000 & 1 \\
\hline 1 & 0.216791000 & 0.190036000 & -0.000098000 & 1 & -0.081465000 & 1.049689000 & 2.149133000 & 1 \\
\hline 8 & -4.164915000 & 1.538314000 & 0.000039000 & 8 & -3.642986000 & -0.797715000 & -0.000012000 & 8 \\
\hline 6 & -3.833322000 & 2.916982000 & 0.000031000 & 6 & -3.641032000 & -2.219127000 & 0.000015000 & 6 \\
\hline 1 & -3.263170000 & 3.192077000 & 0.891330000 & 1 & -3.142678000 & -2.617668000 & -0.889720000 & 1 \\
\hline 1 & -3.263229000 & 3.192080000 & -0.891305000 & 1 & -3.142732000 & -2.617635000 & 0.889794000 & 1 \\
\hline 1 & -4.778835000 & 3.452570000 & 0.000063000 & 1 & -4.682127000 & -2.537014000 & -0.000011000 & 1 \\
\hline & & & & & $n=$ & In & & \\
\hline 6 & -3.262780000 & -1.222828000 & -0.041695000 & 6 & -2.929887000 & -0.763597000 & -0.027289000 & 6 \\
\hline 6 & -1.906366000 & -1.405167000 & 0.160288000 & 6 & -2.627839000 & 0.059780000 & 1.041770000 & 6 \\
\hline 6 & -3.815368000 & 0.030789000 & -0.235054000 & 6 & -2.023607000 & -0.958405000 & -1.052051000 & 6 \\
\hline 1 & -1.514172000 & -2.401290000 & 0.307671000 & 1 & -3.357617000 & 0.215856000 & 1.824180000 & 1 \\
\hline 1 & -4.879889000 & 0.132558000 & -0.389402000 & 1 & -2.288154000 & -1.586597000 & -1.891416000 & 1 \\
\hline 6 & -1.067007000 & -0.296775000 & 0.169935000 & 6 & -1.393053000 & 0.694505000 & 1.097131000 & 6 \\
\hline 6 & -2.978193000 & 1.139840000 & -0.225936000 & 6 & -0.783017000 & -0.331034000 & -1.010195000 & 6 \\
\hline 1 & -0.001650000 & -0.414622000 & 0.326941000 & 1 & -1.171704000 & 1.371367000 & 1.910043000 & 1 \\
\hline 1 & -3.381413000 & 2.130674000 & -0.374607000 & 1 & -0.098088000 & -0.445670000 & -1.836455000 & 1 \\
\hline 6 & -1.616152000 & 0.963586000 & -0.023899000 & 6 & -0.466451000 & 0.508298000 & 0.062788000 & 6 \\
\hline 17 & -0.567173000 & 2.374616000 & -0.014682000 & 17 & 0.406883000 & 2.325894000 & -0.565606000 & 17 \\
\hline 9 & -4.074068000 & -2.301074000 & -0.050448000 & 9 & -4.138593000 & -1.379574000 & -0.076285000 & 9 \\
\hline 6 & 3.526738000 & -0.748315000 & -0.024641000 & 6 & 2.214077000 & -0.477246000 & -0.062908000 & 6 \\
\hline 1 & 3.590857000 & -1.795209000 & 0.283947000 & 1 & 1.794225000 & -1.379377000 & -0.509592000 & 1 \\
\hline 1 & 3.274526000 & -0.760268000 & -1.088229000 & 1 & 2.363953000 & 0.253272000 & -0.856701000 & 1 \\
\hline 6 & 4.890215000 & -0.077785000 & 0.167206000 & 6 & 3.533509000 & -0.792364000 & 0.639568000 & 6 \\
\hline 1 & 5.132643000 & -0.065319000 & 1.234493000 & 1 & 3.353296000 & -1.497462000 & 1.456210000 & 1 \\
\hline 1 & 4.817545000 & 0.969119000 & -0.143115000 & 1 & 3.925547000 & 0.120836000 & 1.095161000 & 1 \\
\hline 6 & 6.009704000 & -0.770557000 & -0.608648000 & 6 & 4.568474000 & -1.372525000 & -0.324109000 & 6 \\
\hline 1 & 6.121129000 & -1.811125000 & -0.296806000 & 1 & 4.213431000 & -2.301241000 & -0.774023000 & 1 \\
\hline 1 & 5.806317000 & -0.768589000 & -1.681490000 & 1 & 4.789287000 & -0.673479000 & -1.132099000 & 1 \\
\hline 1 & 6.968458000 & -0.274812000 & -0.453456000 & 1 & 5.503064000 & -1.589978000 & 0.192317000 & 1 \\
\hline 7 & 2.403547000 & -0.139274000 & 0.692640000 & 7 & 1.189312000 & 0.082235000 & 0.833078000 & 7 \\
\hline 1 & 2.282303000 & 0.828066000 & 0.413275000 & 1 & 1.482367000 & 0.971491000 & 1.226353000 & 1 \\
\hline 1 & 2.582338000 & -0.129792000 & 1.690590000 & 1 & 0.942300000 & -0.563162000 & 1.578100000 & 1 \\
\hline & & & & & $\boldsymbol{n}=$ & D & & \\
\hline 6 & -1.042013000 & -0.959276000 & -0.003629000 & 6 & -1.774719000 & 0.044293000 & 0.000005000 & 6 \\
\hline 6 & -0.774832000 & 0.369065000 & -0.332086000 & 6 & -1.120594000 & 0.331797000 & 1.198166000 & 6 \\
\hline 6 & -2.325409000 & -1.347983000 & 0.361752000 & 6 & -1.120581000 & 0.331758000 & -1.198159000 & 6 \\
\hline 1 & 0.227075000 & 0.674162000 & -0.615610000 & 1 & -1.618417000 & 0.145526000 & 2.139945000 & 1 \\
\hline 1 & -2.529669000 & -2.377560000 & 0.615186000 & 1 & -1.618392000 & 0.145457000 & -2.139939000 & 1 \\
\hline 6 & -1.796763000 & 1.305631000 & -0.294523000 & 6 & 0.148350000 & 0.876216000 & 1.213829000 & 6 \\
\hline 6 & -3.351862000 & -0.413041000 & 0.400506000 & 6 & 0.148362000 & 0.876176000 & -1.213822000 & 6 \\
\hline 1 & -4.352692000 & -0.704723000 & 0.681834000 & 1 & 0.613409000 & 1.140831000 & -2.153091000 & 1 \\
\hline 6 & -3.076277000 & 0.906052000 & 0.071589000 & 6 & 0.813432000 & 1.159706000 & 0.000003000 & 6 \\
\hline 17 & -4.364906000 & 2.089288000 & 0.118398000 & 17 & 1.703942000 & 2.979137000 & -0.000016000 & 17 \\
\hline 6 & 3.448213000 & 1.251953000 & 0.037261000 & 6 & 2.408994000 & -1.082237000 & -0.000011000 & 6 \\
\hline 1 & 3.280304000 & 2.295194000 & 0.318494000 & 1 & 1.833904000 & -1.380548000 & 0.876896000 & 1 \\
\hline 1 & 3.106708000 & 0.648926000 & 0.882089000 & 1 & 1.833972000 & -1.380527000 & -0.876970000 & 1 \\
\hline 6 & 4.945428000 & 1.019688000 & -0.182998000 & 6 & 3.789856000 & -1.731782000 & 0.000033000 & 6 \\
\hline 1 & 5.277113000 & 1.620318000 & -1.035983000 & 1 & 4.350689000 & -1.394847000 & 0.876356000 & 1 \\
\hline 1 & 5.104832000 & -0.026086000 & -0.463143000 & 1 & 4.350764000 & -1.394807000 & -0.876227000 & 1 \\
\hline 6 & 5.787280000 & 1.359296000 & 1.046315000 & 6 & 3.702257000 & -3.257772000 & -0.000006000 & 6 \\
\hline 1 & 5.669537000 & 2.407620000 & 1.328482000 & 1 & 3.172918000 & -3.623297000 & 0.881262000 & 1 \\
\hline 1 & 5.493515000 & 0.752320000 & 1.905057000 & 1 & 3.172969000 & -3.623253000 & -0.881323000 & 1 \\
\hline 1 & 6.847935000 & 1.183263000 & 0.864434000 & 1 & 4.696202000 & -3.704092000 & 0.000011000 & 1 \\
\hline 7 & 2.576458000 & 0.950899000 & -1.102569000 & 7 & 2.453161000 & 0.390287000 & 0.000009000 & 7 \\
\hline 1 & 2.669620000 & -0.022392000 & -1.371704000 & 1 & 2.920364000 & 0.774300000 & -0.816709000 & 1 \\
\hline 1 & 2.845877000 & 1.501225000 & -1.910271000 & 1 & 2.920376000 & 0.774277000 & 0.816730000 & 1 \\
\hline
\end{tabular}




\begin{tabular}{|c|c|c|c|c|c|c|c|c|c|c|c|}
\hline 1 & -1.604063000 & 2.337795000 & -0.546849000 & 1 & 0.613379000 & 1.140910000 & 2.153092000 & 1 & 0.124149000 & 0.842244000 & 2.255028000 \\
\hline 6 & 0.083297000 & -1.954468000 & -0.037059000 & 6 & -3.115767000 & -0.603592000 & 0.000003000 & 6 & -3.737313000 & -0.127106000 & -0.129636000 \\
\hline 9 & -0.331328000 & -3.214431000 & 0.193821000 & 9 & -3.849222000 & -0.276642000 & -1.087729000 & 9 & -4.112075000 & -0.205082000 & -1.422446000 \\
\hline 9 & 0.717286000 & -1.961880000 & -1.233437000 & 9 & -3.041461000 & -1.964912000 & -0.000062000 & 9 & -4.301517000 & -1.186679000 & 0.499839000 \\
\hline 9 & 1.030679000 & -1.677661000 & 0.889663000 & 9 & -3.849186000 & -0.276741000 & 1.087785000 & 9 & -4.317728000 & 0.975299000 & 0.389747000 \\
\hline \multicolumn{12}{|c|}{$n=4, \mathrm{R}=-\mathrm{COOH}$} \\
\hline 6 & 2.703394000 & -0.523965000 & 0.018400000 & 6 & 2.044257000 & -0.151864000 & 0.036723000 & 6 & 2.605101000 & -0.148735000 & 0.062135000 \\
\hline 6 & 1.338030000 & -0.723858000 & -0.192277000 & 6 & 1.417585000 & 0.177207000 & -1.173309000 & 6 & 1.770377000 & -0.739919000 & -0.885416000 \\
\hline 6 & 3.185016000 & 0.768964000 & 0.230270000 & 6 & 1.388642000 & 0.192081000 & 1.227449000 & 6 & 2.030590000 & 0.445991000 & 1.189771000 \\
\hline 1 & 0.961739000 & -1.722374000 & -0.356701000 & 1 & 1.914262000 & -0.049073000 & -2.106010000 & 1 & 2.201315000 & -1.200729000 & -1.761681000 \\
\hline 1 & 4.243723000 & 0.911891000 & 0.392024000 & 1 & 1.876679000 & -0.031100000 & 2.166477000 & 1 & 2.679370000 & 0.903984000 & 1.922556000 \\
\hline 6 & 0.456832000 & 0.348486000 & -0.192465000 & 6 & 0.187868000 & 0.797288000 & -1.206875000 & 6 & 0.394702000 & -0.744940000 & -0.716477000 \\
\hline 6 & 2.318197000 & 1.848815000 & 0.232769000 & 6 & 0.161466000 & 0.813416000 & 1.228285000 & 6 & 0.662732000 & 0.447080000 & 1.363099000 \\
\hline 1 & 2.682402000 & 2.852317000 & 0.396127000 & 1 & -0.301007000 & 1.111525000 & 2.159175000 & 1 & 0.229380000 & 0.916816000 & 2.237488000 \\
\hline 6 & 0.962565000 & 1.624072000 & 0.021030000 & 6 & -0.476956000 & 1.127496000 & 0.000947000 & 6 & -0.177538000 & -0.150770000 & 0.412589000 \\
\hline 17 & -0.133735000 & 2.991803000 & 0.024941000 & 17 & -1.254610000 & 2.972130000 & -0.020330000 & 17 & -2.590758000 & 2.612806000 & -0.680180000 \\
\hline 6 & -3.948745000 & -0.868448000 & 0.006232000 & 6 & -2.150323000 & -1.043370000 & -0.001216000 & 6 & -2.449231000 & -1.069336000 & -0.052799000 \\
\hline 1 & -3.751487000 & -1.861176000 & -0.407039000 & 1 & -1.579504000 & -1.368957000 & -0.870928000 & 1 & -2.485648000 & -0.850162000 & -1.121614000 \\
\hline 1 & -3.667943000 & -0.917660000 & 1.061557000 & 1 & -1.596225000 & -1.358272000 & 0.883083000 & 1 & -2.045983000 & -2.082238000 & 0.058934000 \\
\hline 6 & -5.443702000 & -0.557275000 & -0.108309000 & 6 & -3.557729000 & -1.633005000 & -0.011172000 & 6 & -3.856965000 & -0.998232000 & 0.527797000 \\
\hline 1 & -5.716114000 & -0.505833000 & -1.167163000 & 1 & -4.095346000 & -1.278097000 & -0.894970000 & 1 & -4.238102000 & 0.019132000 & 0.418972000 \\
\hline 1 & -5.631543000 & 0.438350000 & 0.305516000 & 1 & -4.112182000 & -1.267430000 & 0.857715000 & 1 & -3.812074000 & -1.206879000 & 1.601621000 \\
\hline 6 & -6.322761000 & -1.586036000 & 0.601650000 & 6 & -3.534899000 & -3.161277000 & -0.001515000 & 6 & -4.810051000 & -1.984612000 & -0.143537000 \\
\hline 1 & -6.177783000 & -2.584742000 & 0.184556000 & 1 & -3.013471000 & -3.553953000 & -0.875734000 & 1 & -4.903883000 & -1.777528000 & -1.210780000 \\
\hline 1 & -6.088875000 & -1.637210000 & 1.667037000 & 1 & -3.029395000 & -3.542977000 & 0.886760000 & 1 & -4.463084000 & -3.013475000 & -0.031900000 \\
\hline 1 & -7.380666000 & -1.339224000 & 0.507287000 & 1 & -4.546849000 & -3.564960000 & -0.008192000 & 1 & -5.806164000 & -1.921491000 & 0.293699000 \\
\hline 7 & -3.042080000 & 0.076364000 & -0.651998000 & 7 & -2.134354000 & 0.430827000 & -0.010284000 & 7 & -1.567722000 & -0.089091000 & 0.596270000 \\
\hline 1 & -3.168064000 & 1.010702000 & -0.278550000 & 1 & -2.595518000 & 0.835440000 & 0.800210000 & 1 & -1.808820000 & 0.044148000 & 1.569405000 \\
\hline 1 & -3.247137000 & 0.133774000 & -1.643240000 & 1 & -2.580556000 & 0.825324000 & -0.834006000 & 1 & -2.116478000 & 1.509735000 & -0.114546000 \\
\hline 1 & -0.603265000 & 0.195155000 & -0.354111000 & 1 & -0.253823000 & 1.083501000 & -2.151455000 & 1 & -0.228600000 & -1.206124000 & -1.467552000 \\
\hline 6 & 3.677386000 & -1.642160000 & 0.026972000 & 6 & 3.344186000 & -0.816050000 & 0.109541000 & 6 & 4.076204000 & -0.125052000 & -0.074268000 \\
\hline 8 & 4.866019000 & -1.523861000 & 0.203889000 & 8 & 3.928128000 & -1.125424000 & 1.126878000 & 8 & 4.840569000 & 0.368257000 & 0.721326000 \\
\hline 8 & 3.101178000 & -2.851166000 & -0.187783000 & 8 & 3.884957000 & -1.084737000 & -1.116046000 & 8 & 4.516793000 & -0.726663000 & -1.209665000 \\
\hline 1 & 3.818225000 & -3.500518000 & -0.161788000 & 1 & 4.732516000 & -1.513223000 & -0.934328000 & 1 & 5.481286000 & -0.650854000 & -1.196096000 \\
\hline \multicolumn{12}{|c|}{$n=4, \mathrm{R}=-\mathrm{CN}$} \\
\hline 6 & -2.870255000 & -1.106865000 & -0.000969000 & 6 & 2.345893000 & -0.609517000 & -0.000003000 & 6 & -3.029164000 & -0.214392000 & -0.031338000 \\
\hline 6 & -1.473689000 & -1.058368000 & 0.060615000 & 6 & 1.761453000 & -0.182264000 & -1.202315000 & 6 & -2.480517000 & 0.329376000 & 1.137556000 \\
\hline 6 & -3.602599000 & 0.082851000 & -0.069214000 & 6 & 1.761481000 & -0.182229000 & 1.202311000 & 6 & -2.171516000 & -0.750680000 & -0.994787000 \\
\hline 1 & -0.908216000 & -1.977774000 & 0.113244000 & 1 & 2.210092000 & -0.472791000 & -2.142664000 & 1 & -3.133849000 & 0.747825000 & 1.889733000 \\
\hline 1 & -4.681480000 & 0.047382000 & -0.116730000 & 1 & 2.210143000 & -0.472731000 & 2.142658000 & 1 & -2.584745000 & -1.171206000 & -1.900503000 \\
\hline 6 & -0.809905000 & 0.158775000 & 0.054496000 & 6 & 0.637775000 & 0.613770000 & -1.215884000 & 6 & -1.114795000 & 0.331665000 & 1.330421000 \\
\hline 6 & -2.946553000 & 1.302754000 & -0.075743000 & 6 & 0.637804000 & 0.613806000 & 1.215885000 & 6 & -0.800526000 & -0.750891000 & -0.800587000 \\
\hline 1 & -3.503520000 & 2.226325000 & -0.128442000 & 1 & 0.237162000 & 0.969254000 & 2.154997000 & 1 & -0.161513000 & -1.168654000 & -1.563418000 \\
\hline 6 & -1.558391000 & 1.327255000 & -0.013753000 & 6 & 0.040524000 & 1.031198000 & 0.000002000 & 6 & -0.251965000 & -0.209695000 & 0.366925000 \\
\hline 17 & -0.736665000 & 2.872603000 & -0.022615000 & 17 & -0.443805000 & 2.972105000 & -0.000014000 & 17 & 2.125898000 & 2.681094000 & -0.485680000 \\
\hline 6 & 3.679863000 & -0.667388000 & 0.003405000 & 6 & -1.958101000 & -0.854611000 & 0.000033000 & 6 & 2.038949000 & -1.065321000 & -0.123190000 \\
\hline 1 & 3.512494000 & -1.423167000 & 0.775268000 & 1 & -1.455499000 & -1.261803000 & -0.877336000 & 1 & 1.654697000 & -2.090874000 & -0.080260000 \\
\hline 1 & 3.468769000 & -1.155827000 & -0.951448000 & 1 & -1.455597000 & -1.261781000 & 0.877468000 & 1 & 2.081887000 & -0.777906000 & -1.175522000 \\
\hline 6 & 5.144286000 & -0.222201000 & 0.035230000 & 6 & -3.442133000 & -1.208796000 & -0.000046000 & 6 & 3.438936000 & -1.004586000 & 0.476952000 \\
\hline 1 & 5.344814000 & 0.270487000 & 0.991781000 & 1 & -3.922058000 & -0.764459000 & -0.876506000 & 1 & 3.386518000 & -1.278346000 & 1.535693000 \\
\hline 1 & 5.301754000 & 0.535395000 & -0.738836000 & 1 & -3.922178000 & -0.764359000 & 0.876296000 & 1 & 3.802540000 & 0.024078000 & 0.433923000 \\
\hline 6 & 6.123698000 & -1.377633000 & -0.166040000 & 6 & -3.668441000 & -2.720495000 & 0.000023000 & 6 & 4.416667000 & -1.931879000 & -0.241462000 \\
\hline 1 & 6.009065000 & -2.133418000 & 0.613686000 & 1 & -3.225925000 & -3.187126000 & -0.881286000 & 1 & 4.089331000 & -2.971966000 & -0.192726000 \\
\hline 1 & 5.962542000 & -1.869573000 & -1.127463000 & 1 & -3.226059000 & -3.187025000 & 0.881453000 & 1 & 4.515435000 & -1.661335000 & -1.293932000 \\
\hline 1 & 7.157503000 & -1.032013000 & -0.141260000 & 1 & -4.732860000 & -2.952829000 & -0.000045000 & 1 & 5.407495000 & -1.874896000 & 0.208256000 \\
\hline 7 & 2.682807000 & 0.391615000 & 0.192677000 & 7 & -1.706010000 & 0.598626000 & 0.000026000 & 7 & 1.132295000 & -0.144961000 & 0.576660000 \\
\hline 1 & 2.786679000 & 1.106681000 & -0.518984000 & 1 & -2.091055000 & 1.065808000 & 0.816729000 & 1 & 1.671097000 & 1.530093000 & -0.017502000 \\
\hline 1 & 2.824828000 & 0.855397000 & 1.083290000 & 1 & -2.091052000 & 1.065798000 & -0.816685000 & 1 & 1.360275000 & -0.066706000 & 1.558732000 \\
\hline 1 & 0.272373000 & 0.195705000 & 0.101601000 & 1 & 0.237113000 & 0.969193000 & -2.154997000 & 1 & -0.702612000 & 0.761602000 & 2.234599000 \\
\hline 6 & -3.546518000 & -2.365207000 & 0.005707000 & 6 & 3.498928000 & -1.438279000 & -0.000004000 & 6 & -4.441203000 & -0.223327000 & -0.232041000 \\
\hline 7 & -4.091938000 & -3.380715000 & 0.011085000 & 7 & 4.431920000 & -2.119546000 & -0.000007000 & 7 & -5.583156000 & -0.232455000 & -0.392546000 \\
\hline
\end{tabular}


Table S11. Coordinates for the optimized reactants, products and transition states for the $n$ $\mathrm{RPhX}+\mathrm{NH}_{2}{ }^{\mathrm{n}} \mathrm{Pr}$ amination for $\mathrm{X}=\mathrm{Br}$, where $\mathrm{n}=2$ (ortho), 3 (meta) or 4 (para) and $\mathrm{R}=-\mathrm{H},-$ $\mathrm{Me},-\mathrm{OMe},-\mathrm{F},-\mathrm{CF}_{3},-\mathrm{COOH}$, and $-\mathrm{CN}$.

\begin{tabular}{|c|c|c|c|c|c|c|c|c|c|c|c|}
\hline \multicolumn{4}{|c|}{ Reactant } & \multicolumn{4}{|c|}{ Transition State } & & & roduct & \\
\hline & & & & & $\boldsymbol{n}=$ & $2, \mathbf{R}=-$ & & & & & \\
\hline 6 & -3.111412000 & 2.448779000 & -0.017415000 & 6 & -0.903514000 & 3.104103000 & $\begin{array}{l}-0.000333000 \\
\end{array}$ & 6 & 3.995883000 & 0.265414000 & 0.361214000 \\
\hline 6 & -1.729737000 & 2.320074000 & 0.061299000 & 6 & -0.829958000 & 2.397196000 & -1.196097000 & 6 & 3.700816000 & -0.725218000 & -0.568431000 \\
\hline 6 & -3.907690000 & 1.311919000 & -0.092924000 & 6 & -0.830860000 & 2.397454000 & 1.195646000 & 6 & 2.968468000 & 0.970683000 & 0.977372000 \\
\hline 1 & -1.103871000 & 3.200674000 & 0.120047000 & 1 & -0.903927000 & 2.919398000 & -2.141778000 & 1 & 4.496987000 & -1.270710000 & -1.055792000 \\
\hline 1 & -4.983972000 & 1.402794000 & -0.154316000 & 1 & -0.905522000 & 2.919865000 & 2.141157000 & 1 & 3.196973000 & 1.751011000 & 1.689949000 \\
\hline 6 & -1.131338000 & 1.063411000 & 0.065547000 & 6 & -0.678824000 & 1.016180000 & -1.212818000 & 6 & 2.377570000 & -1.015719000 & -0.876342000 \\
\hline 6 & -3.329033000 & 0.047353000 & -0.090030000 & 6 & -0.679741000 & 1.016448000 & 1.212787000 & 6 & 1.640997000 & 0.691873000 & 0.674259000 \\
\hline 1 & -0.055042000 & 0.962574000 & 0.126106000 & 1 & -0.664341000 & 0.479770000 & -2.150880000 & 1 & 2.142017000 & -1.786100000 & -1.600456000 \\
\hline 1 & -3.941733000 & -0.840190000 & -0.148563000 & 1 & -0.665913000 & 0.480241000 & 2.150973000 & 1 & 0.830247000 & 1.255085000 & 1.116358000 \\
\hline 6 & -1.947473000 & -0.055985000 & -0.010599000 & 6 & -0.613909000 & 0.324068000 & 0.000085000 & 6 & 1.362345000 & -0.305111000 & -0.250217000 \\
\hline 35 & -1.149293000 & -1.802573000 & -0.006825000 & 35 & -1.861471000 & -1.563843000 & -0.000142000 & 35 & -1.839821000 & 1.796678000 & -0.133194000 \\
\hline 1 & -3.566216000 & 3.430072000 & -0.019959000 & 1 & -1.027686000 & 4.177233000 & -0.000495000 & 1 & 5.026121000 & 0.491976000 & 0.599226000 \\
\hline 6 & 3.631853000 & 0.824824000 & 0.001454000 & 6 & 2.191795000 & -0.083250000 & 0.001510000 & 6 & -0.674468000 & -1.635715000 & 0.329565000 \\
\hline 1 & 3.591731000 & 1.303587000 & -0.980673000 & 1 & 2.181114000 & 0.568269000 & -0.873514000 & 1 & 0.010525000 & -2.478737000 & 0.424212000 \\
\hline 1 & 3.779013000 & 1.630646000 & 0.725645000 & 1 & 2.181973000 & 0.564833000 & 0.879070000 & 1 & -0.781008000 & -1.160303000 & 1.303384000 \\
\hline 6 & 4.829466000 & -0.128711000 & 0.052932000 & 6 & 3.434960000 & -0.971709000 & -0.000861000 & 6 & -2.027892000 & -2.061771000 & -0.219596000 \\
\hline 1 & 4.675589000 & -0.931294000 & -0.675033000 & 1 & 3.411769000 & -1.624267000 & -0.878005000 & 1 & -1.891576000 & -2.514960000 & -1.207279000 \\
\hline 1 & 4.859212000 & -0.608450000 & 1.036271000 & 1 & 3.412773000 & -1.627633000 & 0.873788000 & 1 & -2.645794000 & -1.172578000 & -0.352102000 \\
\hline 6 & 6.160488000 & 0.570195000 & -0.221110000 & 6 & 4.725619000 & -0.152996000 & -0.000045000 & 6 & -2.722488000 & -3.055339000 & 0.710037000 \\
\hline 1 & 6.167543000 & 1.033205000 & -1.210061000 & 1 & 4.786587000 & 0.489492000 & -0.880043000 & 1 & -2.123101000 & -3.955594000 & 0.860269000 \\
\hline 1 & 6.351457000 & 1.356800000 & 0.511824000 & 1 & 4.787789000 & 0.485854000 & 0.882514000 & 1 & -2.911609000 & -2.609579000 & 1.687437000 \\
\hline 1 & 6.995087000 & -0.130374000 & -0.178718000 & 1 & 5.600382000 & -0.802925000 & -0.001981000 & 1 & -3.682558000 & -3.360703000 & 0.295756000 \\
\hline 7 & 2.320361000 & 0.226517000 & 0.259724000 & 7 & 0.925397000 & -0.822972000 & 0.000624000 & 7 & -0.032011000 & -0.608558000 & -0.560856000 \\
\hline 1 & 2.297466000 & -0.202546000 & 1.178019000 & 1 & 0.792234000 & -1.410264000 & 0.817592000 & 1 & -0.667760000 & 0.324973000 & -0.471044000 \\
\hline 1 & 2.125703000 & -0.514233000 & -0.404683000 & 1 & 0.793058000 & -1.409510000 & -0.817026000 & 1 & -0.109032000 & -0.912169000 & -1.529309000 \\
\hline & & & & & $\boldsymbol{n}=2$ & $\mathbf{R}=$ & & & & & \\
\hline 6 & 2.946713000 & 2.362914000 & -0.341083000 & 6 & 0.960539000 & 3.064142000 & -0.375041000 & 6 & 4.060558000 & -0.152476000 & -0.068527000 \\
\hline 6 & 2.322835000 & 1.938185000 & 0.823720000 & 6 & 0.921190000 & 2.432642000 & 0.862447000 & 6 & 3.181812000 & -0.581147000 & 0.916126000 \\
\hline 6 & 2.957387000 & 1.540445000 & -1.459740000 & 6 & 0.796606000 & 2.297943000 & -1.522041000 & 6 & 3.576475000 & 0.513553000 & -1.185966000 \\
\hline 1 & 2.318860000 & 2.582812000 & 1.693399000 & 1 & 1.056156000 & 3.015786000 & 1.765729000 & 1 & 3.565841000 & -1.112119000 & 1.777315000 \\
\hline 1 & 3.441185000 & 1.857355000 & -2.373584000 & 1 & 0.840178000 & 2.757021000 & -2.501346000 & 1 & 4.246531000 & 0.845757000 & -1.966270000 \\
\hline 6 & 1.690816000 & 0.694315000 & 0.918691000 & 6 & 0.719077000 & 1.058838000 & 1.003183000 & 6 & 1.803710000 & -0.366107000 & 0.833058000 \\
\hline 6 & 2.341097000 & 0.297300000 & -1.402597000 & 6 & 0.595594000 & 0.926456000 & -1.433893000 & 6 & 2.213631000 & 0.743766000 & -1.296147000 \\
\hline 1 & 2.340691000 & -0.356834000 & -2.262057000 & 1 & 0.513818000 & 0.327167000 & -2.329315000 & 1 & 1.821542000 & 1.257016000 & -2.166133000 \\
\hline 6 & 1.721866000 & -0.104095000 & -0.227097000 & 6 & 0.576197000 & 0.321591000 & -0.179065000 & 6 & 1.344235000 & 0.311379000 & -0.301130000 \\
\hline 35 & 0.890628000 & -1.839394000 & -0.217529000 & 35 & 1.772325000 & -1.643341000 & -0.204577000 & 35 & -2.059256000 & -1.721843000 & -0.327003000 \\
\hline 1 & 3.424153000 & 3.332971000 & -0.372897000 & 1 & 1.123890000 & 4.130299000 & -0.440315000 & 1 & 5.119480000 & -0.346667000 & 0.033452000 \\
\hline 6 & -3.429851000 & 0.718038000 & 0.486771000 & 6 & -2.264761000 & -0.059368000 & -0.029334000 & 6 & -0.666978000 & 1.689235000 & 0.338729000 \\
\hline 1 & -3.934804000 & 1.274631000 & 1.281330000 & 1 & -2.249526000 & 0.552434000 & 0.873775000 & 1 & -0.780780000 & 1.296120000 & 1.346087000 \\
\hline 1 & -2.841506000 & 1.452341000 & -0.069962000 & 1 & -2.244788000 & 0.628895000 & -0.876033000 & 1 & 0.053371000 & 2.507350000 & 0.363493000 \\
\hline 6 & -4.478649000 & 0.098717000 & -0.442394000 & 6 & -3.523635000 & -0.925462000 & -0.070555000 & 6 & -2.010158000 & 2.137743000 & -0.220297000 \\
\hline 1 & -5.057752000 & -0.640879000 & 0.119748000 & 1 & -3.516565000 & -1.615760000 & 0.777389000 & 1 & -2.667569000 & 1.270374000 & -0.294204000 \\
\hline 1 & -3.967653000 & -0.452334000 & -1.237886000 & 1 & -3.505576000 & -1.543103000 & -0.972806000 & 1 & -1.867652000 & 2.522232000 & -1.235714000 \\
\hline 6 & -5.420285000 & 1.135231000 & -1.054090000 & 6 & -4.800839000 & -0.086459000 & -0.044212000 & 6 & -2.647651000 & 3.217803000 & 0.651859000 \\
\hline 1 & -5.961968000 & 1.682424000 & -0.279549000 & 1 & -4.858337000 & 0.518127000 & 0.862560000 & 1 & -2.844194000 & 2.844424000 & 1.657918000 \\
\hline 1 & -4.869580000 & 1.866664000 & -1.649155000 & 1 & -4.845872000 & 0.591188000 & -0.898440000 & 1 & -2.007131000 & 4.097892000 & 0.738463000 \\
\hline 1 & -6.158969000 & 0.668077000 & -1.706357000 & 1 & -5.686186000 & -0.721121000 & -0.076586000 & 1 & -3.598592000 & 3.539313000 & 0.228862000 \\
\hline 7 & -2.495284000 & -0.214195000 & 1.118811000 & 7 & -1.011482000 & -0.817330000 & -0.065484000 & 7 & -0.080330000 & 0.583626000 & -0.495703000 \\
\hline 1 & -1.970257000 & -0.728858000 & 0.420415000 & 1 & -0.905459000 & -1.394135000 & -0.892802000 & 1 & -0.221511000 & 0.821610000 & -1.475568000 \\
\hline 1 & -2.995355000 & -0.901940000 & 1.670567000 & 1 & -0.862577000 & -1.416057000 & 0.739044000 & 1 & -0.736688000 & -0.331048000 & -0.375373000 \\
\hline 6 & 1.016098000 & 0.275886000 & 2.193847000 & 6 & 0.702807000 & 0.415288000 & 2.360574000 & 6 & 0.899746000 & -0.858541000 & 1.932293000 \\
\hline 1 & -0.063517000 & 0.178559000 & 2.055645000 & 1 & -0.260369000 & -0.052093000 & 2.589262000 & 1 & 0.660272000 & -0.056779000 & 2.635828000 \\
\hline 1 & 1.387591000 & -0.689979000 & 2.539606000 & 1 & 1.460320000 & -0.367065000 & 2.427533000 & 1 & -0.036599000 & -1.264217000 & 1.547952000 \\
\hline 1 & 1.195703000 & 1.011513000 & 2.976424000 & 1 & 0.893269000 & 1.152981000 & 3.138548000 & 1 & 1.397668000 & -1.643392000 & 2.498945000 \\
\hline & & & & & $n=2$ & $\mathbf{D}_{-}$ & & & & & \\
\hline 6 & 0.231424000 & 2.712780000 & 0.684952000 & 6 & -0.370620000 & 2.616447000 & -1.402878000 & 6 & 2.564234000 & -2.072198000 & -1.332229000 \\
\hline 6 & 0.087261000 & 1.492302000 & 1.335162000 & 6 & -0.314643000 & 2.340103000 & -0.033560000 & 6 & 2.865865000 & -1.342923000 & -0.185429000 \\
\hline 6 & 1.088534000 & 2.840042000 & -0.397473000 & 6 & -0.050071000 & 1.621273000 & -2.308591000 & 6 & 1.318665000 & -1.969819000 & -1.933663000 \\
\hline 1 & -0.591572000 & 1.409209000 & 2.169504000 & 1 & -0.576465000 & 3.110291000 & 0.677665000 & 1 & 3.836962000 & -1.443175000 & 0.274159000 \\
\hline 1 & 1.202952000 & 3.788168000 & -0.903702000 & 1 & -0.076168000 & 1.816503000 & -3.372467000 & 1 & 1.083678000 & -2.554641000 & -2.811072000 \\
\hline 6 & 0.805804000 & 0.372391000 & 0.910856000 & 6 & 0.064852000 & 1.087965000 & 0.424171000 & 6 & 1.910379000 & -0.490998000 & 0.359480000 \\
\hline 6 & 1.807228000 & 1.731311000 & -0.833227000 & 6 & 0.343525000 & 0.356611000 & -1.866610000 & 6 & 0.359198000 & -1.123528000 & -1.389170000 \\
\hline 1 & 2.479930000 & 1.808004000 & -1.675038000 & 1 & 0.652771000 & -0.395923000 & -2.577963000 & 1 & -0.634261000 & -1.061637000 & -1.811889000 \\
\hline 6 & 1.666567000 & 0.514945000 & -0.187892000 & 6 & 0.433754000 & 0.098473000 & -0.505800000 & 6 & 0.660941000 & -0.379892000 & -0.261671000 \\
\hline 35 & 2.660979000 & -0.992802000 & -0.806379000 & 35 & 2.339960000 & -0.971049000 & 0.056114000 & 35 & -3.071391000 & -0.813372000 & 0.443314000 \\
\hline 6 & -3.227404000 & -0.639920000 & -0.769906000 & 6 & -1.893072000 & -1.620440000 & -0.477162000 & 6 & -0.668254000 & 1.746663000 & -0.411282000 \\
\hline 1 & -2.712942000 & 0.321011000 & -0.845289000 & 1 & -1.925126000 & -1.129733000 & -1.454393000 & 1 & -0.927515000 & 1.451580000 & -1.426177000 \\
\hline 1 & -3.290356000 & -1.042602000 & -1.791270000 & 1 & -2.166795000 & -2.668165000 & -0.629077000 & 1 & -1.576865000 & 2.129790000 & 0.052863000 \\
\hline 6 & -4.638550000 & -0.415949000 & -0.237354000 & 6 & -2.877205000 & -0.955600000 & 0.477879000 & 6 & 0.453185000 & 2.773513000 & -0.395011000 \\
\hline 1 & -4.568280000 & 0.001701000 & 0.769656000 & 1 & -2.591400000 & 0.087503000 & 0.617069000 & 1 & 1.356167000 & 2.339798000 & -0.829705000 \\
\hline 1 & -5.138150000 & -1.384907000 & -0.132952000 & 1 & -2.805690000 & -1.436668000 & 1.458021000 & 1 & 0.696016000 & 3.030922000 & 0.639385000 \\
\hline 6 & -5.475481000 & 0.495936000 & -1.132122000 & 6 & -4.314230000 & -1.037299000 & -0.032588000 & 6 & 0.063425000 & 4.035958000 & -1.163832000 \\
\hline 1 & -5.016799000 & 1.482146000 & -1.228900000 & 1 & -4.420033000 & -0.533091000 & -0.994525000 & 1 & -0.149715000 & 3.811833000 & -2.210230000 \\
\hline 1 & -5.580006000 & 0.081411000 & -2.136898000 & 1 & -4.633370000 & -2.073342000 & -0.162207000 & 1 & -0.825265000 & 4.502982000 & -0.736489000 \\
\hline 1 & -6.478211000 & 0.636402000 & -0.727140000 & 1 & -5.003571000 & -0.563925000 & 0.666331000 & 1 & 0.869144000 & 4.769109000 & -1.139053000 \\
\hline 7 & -2.450363000 & -1.488652000 & 0.140191000 & 7 & -0.504850000 & -1.535255000 & -0.004587000 & 7 & -0.345650000 & 0.482300000 & 0.328686000 \\
\hline 1 & -1.498437000 & -1.592457000 & -0.191448000 & 1 & 0.111723000 & -2.239767000 & -0.390667000 & 1 & -1.341069000 & -0.076086000 & 0.416673000 \\
\hline 1 & -2.847082000 & -2.421147000 & 0.174424000 & 1 & -0.404622000 & -1.539913000 & 1.004902000 & 1 & -0.056022000 & 0.711757000 & 1.277826000 \\
\hline 1 & -0.335828000 & 3.565447000 & 1.033120000 & 1 & -0.663055000 & 3.600295000 & -1.740472000 & 1 & 3.313635000 & -2.732215000 & -1.747263000 \\
\hline 8 & 0.726122000 & -0.848699000 & 1.485957000 & 8 & 0.089428000 & 0.702559000 & 1.738236000 & 8 & 2.081296000 & 0.266543000 & 1.480857000 \\
\hline
\end{tabular}




\begin{tabular}{|c|c|c|c|c|c|c|c|c|c|c|}
\hline 6 & -0.099485000 & -1.012822000 & 2.637117000 & 6 & -0.067115000 & 1.700081000 & 2.732806000 & 6 & 3.284719000 & 0.120928000 \\
\hline 1 & 0.210935000 & -0.341759000 & 3.441315000 & 1 & 0.680997000 & 2.488320000 & 2.621387000 & 1 & 3.417035000 & -0.910037000 \\
\hline 1 & -1.149357000 & -0.856395000 & 2.385302000 & 1 & -1.065884000 & 2.145302000 & 2.705599000 & 1 & 4.152114000 & 0.432518000 \\
\hline 1 & 0.047096000 & -2.042102000 & 2.952933000 & 1 & 0.075553000 & 1.199662000 & 3.687027000 & 1 & 3.179700000 & 0.770850000 \\
\hline & & & & & $n=$ & $\mathrm{R}=$ & & & & \\
\hline 6 & 2.473399000 & 2.625022000 & -0.176913000 & 6 & 0.957610000 & 3.051771000 & -0.258154000 & 6 & 3.839835000 & -0.000352000 \\
\hline 6 & 1.579067000 & 2.163944000 & 0.779593000 & 6 & 0.849161000 & 2.379866000 & 0.958735000 & 6 & 3.423666000 & -0.209592000 \\
\hline 6 & 3.040593000 & 1.739500000 & -1.086011000 & 6 & 0.857604000 & 2.321594000 & -1.434844000 & 6 & 2.932031000 & 0.463526000 \\
\hline 1 & 1.116841000 & 2.826967000 & 1.496794000 & 1 & 0.913955000 & 2.898276000 & 1.906016000 & 1 & 4.098254000 & -0.566396000 \\
\hline 1 & 3.736633000 & 2.095027000 & -1.832794000 & 1 & 0.950222000 & 2.817691000 & -2.392072000 & 1 & 3.247276000 & 0.627436000 \\
\hline 6 & 1.262970000 & 0.817533000 & 0.816245000 & 6 & 0.648434000 & 1.018360000 & 0.971788000 & 6 & 2.111752000 & 0.047580000 \\
\hline 6 & 2.715456000 & 0.389546000 & -1.042128000 & 6 & 0.663936000 & 0.943025000 & -1.418214000 & 6 & 1.611105000 & 0.719821000 \\
\hline 1 & 3.149518000 & -0.305471000 & -1.745894000 & 1 & 0.642712000 & 0.386272000 & -2.344395000 & 1 & 0.916539000 & 1.073165000 \\
\hline 6 & 1.821211000 & -0.075987000 & -0.086828000 & 6 & 0.580444000 & 0.261992000 & -0.201012000 & 6 & 1.173680000 & 0.513849000 \\
\hline 35 & 1.355604000 & -1.918784000 & -0.011691000 & 35 & 1.791820000 & -1.612008000 & -0.148717000 & 35 & -1.755217000 & -2.027988000 \\
\hline 6 & -3.540569000 & 0.777475000 & -0.489066000 & 6 & -2.232413000 & -0.068923000 & -0.104007000 & 6 & -0.992967000 & 1.697732000 \\
\hline 1 & -3.712929000 & 1.745042000 & -0.008691000 & 1 & -2.213904000 & 0.616502000 & 0.743867000 & 1 & -0.423906000 & 2.620139000 \\
\hline 1 & -3.686431000 & 0.943661000 & -1.560394000 & 1 & -2.211288000 & 0.541147000 & -1.008453000 & 1 & -1.263807000 & 1.306422000 \\
\hline 6 & -4.584009000 & -0.226882000 & 0.011951000 & 6 & -3.488456000 & -0.938217000 & -0.065737000 & 6 & -2.258267000 & 1.987822000 \\
\hline 1 & -4.436165000 & -0.386046000 & 1.084673000 & 1 & -3.473504000 & -1.555688000 & 0.836318000 & 1 & -1.979414000 & 2.367074000 \\
\hline 1 & -4.403799000 & -1.193818000 & -0.468382000 & 1 & -3.476598000 & -1.627549000 & -0.914540000 & 1 & -2.801160000 & 1.054466000 \\
\hline 6 & -6.020838000 & 0.221720000 & -0.251455000 & 6 & -4.766461000 & -0.100588000 & -0.098075000 & 6 & -3.161887000 & 3.000726000 \\
\hline 1 & -6.233779000 & 1.173255000 & 0.240536000 & 1 & -4.817133000 & 0.575544000 & 0.756938000 & 1 & -2.649080000 & 3.951627000 \\
\hline 1 & -6.202690000 & 0.356799000 & -1.319844000 & 1 & -4.819936000 & 0.504779000 & -1.004476000 & 1 & -3.486268000 & 2.631780000 \\
\hline 1 & -6.742356000 & -0.508787000 & 0.116354000 & 1 & -5.650538000 & -0.737106000 & -0.070949000 & 1 & -4.055133000 & 3.199272000 \\
\hline 7 & -2.139404000 & 0.416760000 & -0.278476000 & 7 & -0.979969000 & -0.832096000 & -0.070311000 & 7 & -0.162307000 & 0.695178000 \\
\hline 1 & -1.926381000 & -0.464309000 & -0.732074000 & 1 & -0.875110000 & -1.487488000 & -0.837534000 & 1 & -0.986006000 & -0.790795000 \\
\hline 1 & -1.946207000 & 0.282637000 & 0.707276000 & 1 & -0.833840000 & -1.341337000 & 0.795369000 & 1 & -0.216019000 & 0.788299000 \\
\hline 9 & 0.401179000 & 0.378635000 & 1.748934000 & 9 & 0.497064000 & 0.369012000 & 2.156273000 & 9 & 1.692062000 & -0.141811000 \\
\hline 1 & 2.723117000 & 3.676241000 & -0.211786000 & 1 & 1.118314000 & 4.119506000 & -0.278156000 & 1 & 4.865235000 & -0.199518000 \\
\hline & & & & & $\boldsymbol{n}=$ & & & & & \\
\hline 6 & 0.259865000 & 2.699410000 & -0.055240000 & 6 & 0.615186000 & 1.915621000 & -2.485351000 & 6 & 3.063119000 & $\begin{array}{c}-0.370046000 \\
\end{array}$ \\
\hline 6 & -0.149257000 & 1.380716000 & -0.199611000 & 6 & 0.784503000 & 2.094313000 & -1.126327000 & 6 & 3.076826000 & -0.004475000 \\
\hline 6 & 1.597251000 & 2.990475000 & 0.168676000 & 6 & 0.302485000 & 0.637335000 & -2.950671000 & 6 & 1.894811000 & -0.882803000 \\
\hline 1 & -1.195190000 & 1.165205000 & -0.376375000 & 1 & 1.038936000 & 3.070222000 & -0.738358000 & 1 & 3.977758000 & 0.392889000 \\
\hline 1 & 1.925586000 & 4.014804000 & 0.282924000 & 1 & 0.182303000 & 0.458684000 & -4.011714000 & 1 & 1.862302000 & -1.172981000 \\
\hline 6 & 0.773672000 & 0.337572000 & -0.122443000 & 6 & 0.642986000 & 1.042820000 & -0.213865000 & 6 & 1.943318000 & -0.143513000 \\
\hline 6 & 2.528462000 & 1.963067000 & 0.247860000 & 6 & 0.160534000 & -0.425248000 & -2.083212000 & 6 & 0.755511000 & -1.029613000 \\
\hline 1 & 3.571764000 & 2.179787000 & 0.421785000 & 1 & -0.034447000 & -1.416691000 & -2.467055000 & 1 & -0.143276000 & -1.422608000 \\
\hline 6 & 2.119750000 & 0.644452000 & 0.103146000 & 6 & 0.334904000 & -0.252091000 & -0.692677000 & 6 & 0.751095000 & -0.663376000 \\
\hline 35 & 3.477841000 & -0.696249000 & 0.228409000 & 35 & 1.511302000 & -1.900896000 & 0.141448000 & 35 & -2.170989000 & 1.996341000 \\
\hline 6 & -4.420991000 & 0.422756000 & 0.227995000 & 6 & -2.405760000 & -0.149251000 & -0.078324000 & 6 & -1.463209000 & -1.732672000 \\
\hline 1 & -3.811922000 & -0.242881000 & 0.844599000 & 1 & -2.212548000 & 0.853028000 & 0.304104000 & 1 & -1.010689000 & -2.697913000 \\
\hline 1 & -4.649884000 & 1.291791000 & 0.850909000 & 1 & -2.523028000 & -0.065904000 & -1.159707000 & 1 & -1.984720000 & -1.373355000 \\
\hline 6 & -5.722839000 & -0.286362000 & -0.154508000 & 6 & -3.662921000 & -0.736014000 & 0.561090000 & 6 & -2.463406000 & -1.903970000 \\
\hline 1 & -5.484285000 & -1.154966000 & -0.776093000 & 1 & -3.510279000 & -0.827464000 & 1.639646000 & 1 & -1.934513000 & -2.250432000 \\
\hline 1 & -6.322303000 & 0.383265000 & -0.779516000 & 1 & -3.823410000 & -1.749205000 & 0.181966000 & 1 & -2.896360000 & -0.932100000 \\
\hline 6 & -6.539112000 & -0.728874000 & 1.059129000 & 6 & -4.898205000 & 0.120481000 & 0.285075000 & 6 & -3.572902000 & -2.892122000 \\
\hline 1 & -5.971770000 & -1.420658000 & 1.684998000 & 1 & -4.775874000 & 1.129489000 & 0.682040000 & 1 & -3.168554000 & -3.879655000 \\
\hline 1 & -6.817788000 & 0.124613000 & 1.680805000 & 1 & -5.090300000 & 0.205056000 & -0.785854000 & 1 & -4.141596000 & -2.553398000 \\
\hline 1 & -7.458342000 & -1.232840000 & 0.758997000 & 1 & -5.784018000 & -0.313291000 & 0.748326000 & 1 & -4.271041000 & -3.004783000 \\
\hline 7 & -3.586324000 & 0.880250000 & -0.887300000 & 7 & -1.194531000 & -0.943914000 & 0.165046000 & 7 & -0.424227000 & -0.759019000 \\
\hline 1 & -4.105480000 & 1.519159000 & -1.479047000 & 1 & -1.254681000 & -1.889987000 & -0.197556000 & 1 & -1.326153000 & 0.842069000 \\
\hline 1 & -3.321905000 & 0.095210000 & -1.472179000 & 1 & -0.943787000 & -0.996105000 & 1.146662000 & 1 & -0.252341000 & -0.767271000 \\
\hline 1 & -0.471908000 & 3.492521000 & -0.118573000 & 1 & 0.733393000 & 2.743578000 & -3.168148000 & 1 & 3.951836000 & -0.255820000 \\
\hline 6 & 0.286289000 & -1.083279000 & -0.285933000 & 6 & 0.913005000 & 1.325819000 & 1.231758000 & 6 & 2.005786000 & 0.266826000 \\
\hline 9 & 0.855590000 & -1.699891000 & -1.340550000 & 9 & 0.077440000 & 0.631888000 & 2.068233000 & 9 & 1.667419000 & -0.761104000 \\
\hline 9 & 0.536473000 & -1.837968000 & 0.801749000 & 9 & 2.163215000 & 1.016388000 & 1.623246000 & 9 & 1.145126000 & 1.269115000 \\
\hline 9 & -1.048206000 & -1.134100000 & -0.487963000 & 9 & 0.730853000 & 2.628907000 & 1.534595000 & 9 & 3.225884000 & 0.675449000 \\
\hline & & & & & $n=2$ & & $\mathbf{O H}$ & & & \\
\hline 6 & 0.264913000 & 2.588076000 & -0.399276000 & 6 & 0.832494000 & 2.767516000 & -1.345754000 & 6 & -2.948379000 & -2.095718000 \\
\hline 6 & -0.112530000 & 1.264724000 & -0.553873000 & 6 & 0.899317000 & 2.416286000 & -0.017801000 & 6 & -3.230426000 & -0.927556000 \\
\hline 6 & 1.561476000 & 2.893511000 & -0.006585000 & 6 & 0.584681000 & 1.759315000 & -2.286721000 & 6 & -1.629023000 & -2.422380000 \\
\hline 1 & -1.127102000 & 1.021361000 & -0.843905000 & 1 & 1.095829000 & 3.155411000 & 0.746433000 & 1 & -4.247092000 & -0.658211000 \\
\hline 1 & 1.868962000 & 3.922675000 & 0.122125000 & 1 & 0.542837000 & 2.000537000 & -3.341635000 & 1 & -1.393986000 & -3.341535000 \\
\hline 6 & 0.799208000 & 0.221527000 & -0.343911000 & 6 & 0.714559000 & 1.092249000 & 0.423693000 & 6 & -2.211154000 & -0.057003000 \\
\hline 6 & 2.472202000 & 1.875322000 & 0.238494000 & 6 & 0.421102000 & 0.447135000 & -1.906490000 & 6 & -0.599683000 & -1.576319000 \\
\hline 1 & 3.473688000 & 2.105454000 & 0.569690000 & 1 & 0.292979000 & -0.323186000 & -2.653553000 & 1 & 0.434521000 & -1.836315000 \\
\hline 6 & 2.097940000 & 0.547783000 & 0.067570000 & 6 & 0.493195000 & 0.074316000 & -0.542361000 & 6 & -0.884426000 & -0.398610000 \\
\hline 35 & 3.422754000 & -0.757665000 & 0.520707000 & 35 & 1.659392000 & -1.716684000 & -0.335067000 & 35 & 2.703836000 & -1.278890000 \\
\hline 6 & -4.502034000 & 0.498487000 & 0.013292000 & 6 & -2.312085000 & -0.084727000 & -0.218740000 & 6 & 0.750703000 & 1.320735000 \\
\hline 1 & -3.985815000 & 0.720681000 & 0.951148000 & 1 & -2.189621000 & 0.766044000 & 0.452638000 & 1 & -0.107133000 & 1.846943000 \\
\hline 1 & -5.056853000 & 1.403745000 & -0.250358000 & 1 & -2.351391000 & 0.312014000 & -1.234623000 & 1 & 1.160417000 & 0.655965000 \\
\hline 6 & -5.488713000 & -0.651274000 & 0.240657000 & 6 & -3.593414000 & -0.848850000 & 0.112978000 & 6 & 1.811398000 & 2.285257000 \\
\hline 1 & -4.926273000 & -1.551819000 & 0.504124000 & 1 & -3.519491000 & -1.253673000 & 1.125759000 & 1 & 1.374759000 & 2.927092000 \\
\hline 1 & -5.998582000 & -0.872226000 & -0.702596000 & 1 & -3.687053000 & -1.706618000 & -0.558878000 & 1 & 2.610118000 & 1.708783000 \\
\hline 6 & -6.520473000 & -0.347980000 & 1.326324000 & 6 & -4.834860000 & 0.035134000 & -0.001374000 & 6 & 2.372403000 & 3.144783000 \\
\hline 1 & -6.037464000 & -0.153699000 & 2.286308000 & 1 & -4.780355000 & 0.882825000 & 0.683586000 & 1 & 1.591622000 & 3.733030000 \\
\hline 1 & -7.114319000 & 0.532998000 & 1.073022000 & 1 & -4.947106000 & 0.431268000 & -1.012042000 & 1 & 2.851630000 & 2.526695000 \\
\hline 1 & -7.209866000 & -1.181354000 & 1.466856000 & 1 & -5.737511000 & -0.527002000 & 0.237048000 & 1 & 3.121535000 & 3.837989000 \\
\hline 7 & -3.482308000 & 0.275523000 & -1.012454000 & 7 & -1.095224000 & -0.894707000 & -0.112264000 & 7 & 0.256214000 & 0.436229000 \\
\hline 1 & -3.916039000 & 0.072414000 & -1.905715000 & 1 & -1.081291000 & -1.694338000 & -0.735914000 & 1 & 1.128946000 & -0.218119000 \\
\hline 1 & -2.903318000 & -0.522819000 & -0.773585000 & 1 & -0.900397000 & -1.205091000 & 0.835568000 & 1 & 0.020894000 & 1.025084000 \\
\hline 1 & -0.454381000 & 3.374362000 & -0.580707000 & 1 & 0.975124000 & 3.791945000 & -1.656134000 & 1 & -3.752927000 & -2.753145000 \\
\hline 6 & 0.266545000 & -1.152683000 & -0.580064000 & 6 & 0.777775000 & 0.882198000 & 1.873994000 & 6 & -2.665519000 & 1.150710000 \\
\hline 8 & -0.877393000 & -1.477993000 & -0.360310000 & 8 & 1.193382000 & 1.671987000 & 2.689440000 & 8 & -3.815132000 & 1.438681000 \\
\hline 8 & 1.156770000 & -2.003828000 & -1.120473000 & 8 & 0.255153000 & -0.319710000 & 2.289232000 & 8 & -1.653104000 & 1.941168000 \\
\hline 1 & 0.700910000 & -2.852563000 & -1.223499000 & 1 & 0.390453000 & -0.344878000 & 3.247011000 & 1 & -2.070531000 & 2.671099000 \\
\hline
\end{tabular}




\begin{tabular}{|c|c|c|c|c|c|c|c|c|}
\hline 6 & 2.096735000 & 2.838577000 & -0.192236000 & 6 & 0.810453000 & 3.075853000 & -0.302430000 & 6 \\
\hline 6 & 1.359297000 & 2.216295000 & 0.800259000 & 6 & 0.755556000 & 2.382008000 & 0.888310000 & 6 \\
\hline 6 & 2.692357000 & 2.076255000 & -1.190621000 & 6 & 0.730516000 & 2.353706000 & -1.497779000 & 6 \\
\hline 1 & 0.887652000 & 2.793872000 & 1.582169000 & 1 & 0.809313000 & 2.902757000 & 1.834834000 & 1 \\
\hline 1 & 3.269231000 & 2.555019000 & -1.970081000 & 1 & 0.784273000 & 2.874063000 & -2.445699000 & 1 \\
\hline 6 & 1.207169000 & 0.823947000 & 0.808055000 & 6 & 0.628700000 & 0.984161000 & 0.916830000 & 6 \\
\hline 6 & 2.553260000 & 0.694165000 & -1.198272000 & 6 & 0.609663000 & 0.979271000 & -1.511208000 & 6 \\
\hline 1 & 3.014659000 & 0.100277000 & -1.972931000 & 1 & 0.604695000 & 0.443136000 & -2.449505000 & 1 \\
\hline 6 & 1.814420000 & 0.070443000 & -0.204054000 & 6 & 0.574252000 & 0.251526000 & -0.301066000 & 6 \\
\hline 35 & 1.643323000 & -1.822400000 & -0.237142000 & 35 & 1.831842000 & -1.495592000 & -0.381839000 & 35 \\
\hline 1 & 2.205322000 & 3.913870000 & -0.187678000 & 1 & 0.917096000 & 4.150132000 & -0.310262000 & 1 \\
\hline 6 & -3.398346000 & 0.438399000 & -0.497040000 & 6 & -2.211592000 & -0.089192000 & -0.236027000 & 6 \\
\hline 1 & -3.385137000 & 1.362854000 & 0.087164000 & 1 & -2.184840000 & 0.565239000 & 0.635280000 & 1 \\
\hline 1 & -3.388581000 & 0.744568000 & -1.547380000 & 1 & -2.211646000 & 0.548596000 & -1.120883000 & 1 \\
\hline 6 & -4.695622000 & -0.323467000 & -0.206183000 & 6 & -3.452335000 & -0.979386000 & -0.203686000 & 6 \\
\hline 1 & -4.701196000 & -0.624637000 & 0.845689000 & 1 & -3.412078000 & -1.622267000 & 0.679389000 & 1 \\
\hline 1 & -4.700427000 & -1.249271000 & -0.790262000 & 1 & -3.446347000 & -1.642073000 & -1.073614000 & 1 \\
\hline 6 & -5.950400000 & 0.490807000 & -0.518620000 & 6 & -4.742304000 & -0.159865000 & -0.186956000 & 6 \\
\hline 1 & -5.984705000 & 1.406372000 & 0.075709000 & 1 & -4.786346000 & 0.488235000 & 0.689622000 & 1 \\
\hline 1 & -5.981748000 & 0.780826000 & -1.571171000 & 1 & -4.822319000 & 0.471543000 & -1.073426000 & 1 \\
\hline 1 & -6.857921000 & -0.075099000 & -0.305032000 & 1 & -5.615629000 & -0.810946000 & -0.162724000 & 1 \\
\hline 7 & -2.152441000 & -0.278031000 & -0.225377000 & 7 & -0.948203000 & -0.841925000 & -0.247051000 & 7 \\
\hline 1 & -2.116673000 & -1.141869000 & -0.754378000 & 1 & -0.851973000 & -1.455208000 & -1.050515000 & 1 \\
\hline 1 & -2.092316000 & -0.535621000 & 0.753418000 & 1 & -0.817497000 & -1.405987000 & 0.589140000 & 1 \\
\hline 6 & 0.437921000 & 0.220982000 & 1.847526000 & 6 & 0.502681000 & 0.303565000 & 2.155996000 & 6 \\
\hline 7 & -0.188302000 & -0.225625000 & 2.705641000 & 7 & 0.331818000 & -0.268273000 & 3.145740000 & 7 \\
\hline & & & & & $n=$ &, $\mathbf{R}=-$ & & \\
\hline 6 & -3.111412000 & 2.448779000 & -0.017415000 & 6 & -0.903514000 & 3.104103000 & -0.000333000 & 6 \\
\hline 6 & -1.729737000 & 2.320074000 & 0.061299000 & 6 & -0.829958000 & 2.397196000 & -1.196097000 & 6 \\
\hline 6 & -3.907690000 & 1.311919000 & -0.092924000 & 6 & -0.830860000 & 2.397454000 & 1.195646000 & 6 \\
\hline 1 & -1.103871000 & 3.200674000 & 0.120047000 & 1 & -0.903927000 & 2.919398000 & -2.141778000 & 1 \\
\hline 1 & -4.983972000 & 1.402794000 & -0.154316000 & 1 & -0.905522000 & 2.919865000 & 2.141157000 & 1 \\
\hline 6 & -1.131338000 & 1.063411000 & 0.065547000 & 6 & -0.678824000 & 1.016180000 & -1.212818000 & 6 \\
\hline 6 & -3.329033000 & 0.047353000 & -0.090030000 & 6 & -0.679741000 & 1.016448000 & 1.212787000 & 6 \\
\hline 1 & -0.055042000 & 0.962574000 & 0.126106000 & 1 & -0.664341000 & 0.479770000 & -2.150880000 & 1 \\
\hline 1 & -3.941733000 & -0.840190000 & -0.148563000 & 1 & -0.665913000 & 0.480241000 & 2.150973000 & 1 \\
\hline 6 & -1.947473000 & -0.055985000 & -0.010599000 & 6 & -0.613909000 & 0.324068000 & 0.000085000 & 6 \\
\hline 35 & -1.149293000 & -1.802573000 & -0.006825000 & 35 & -1.861471000 & -1.563843000 & -0.000142000 & 35 \\
\hline 1 & -3.566216000 & 3.430072000 & -0.019959000 & 1 & -1.027686000 & 4.177233000 & -0.000495000 & 1 \\
\hline 6 & 3.631853000 & 0.824824000 & 0.001454000 & 6 & 2.191795000 & -0.083250000 & 0.001510000 & 6 \\
\hline 1 & 3.591731000 & 1.303587000 & -0.980673000 & 1 & 2.181114000 & 0.568269000 & -0.873514000 & 1 \\
\hline 1 & 3.779013000 & 1.630646000 & 0.725645000 & 1 & 2.181973000 & 0.564833000 & 0.879070000 & 1 \\
\hline 6 & 4.829466000 & -0.128711000 & 0.052932000 & 6 & 3.434960000 & -0.971709000 & -0.000861000 & 6 \\
\hline 1 & 4.675589000 & -0.931294000 & -0.675033000 & 1 & 3.411769000 & -1.624267000 & -0.878005000 & 1 \\
\hline 1 & 4.859212000 & -0.608450000 & 1.036271000 & 1 & 3.412773000 & -1.627633000 & 0.873788000 & 1 \\
\hline 6 & 6.160488000 & 0.570195000 & -0.221110000 & 6 & 4.725619000 & -0.152996000 & -0.000045000 & 6 \\
\hline 1 & 6.167543000 & 1.033205000 & -1.210061000 & 1 & 4.786587000 & 0.489492000 & -0.880043000 & 1 \\
\hline 1 & 6.351457000 & 1.356800000 & 0.511824000 & 1 & 4.787789000 & 0.485854000 & 0.882514000 & 1 \\
\hline 1 & 6.995087000 & -0.130374000 & -0.178718000 & 1 & 5.600382000 & -0.802925000 & -0.001981000 & 1 \\
\hline 7 & 2.320361000 & 0.226517000 & 0.259724000 & 7 & 0.925397000 & -0.822972000 & 0.000624000 & 7 \\
\hline 1 & 2.297466000 & -0.202546000 & 1.178019000 & 1 & 0.792234000 & -1.410264000 & 0.817592000 & 1 \\
\hline 1 & 2.125703000 & -0.514233000 & -0.404683000 & 1 & 0.793058000 & -1.409510000 & -0.817026000 & 1 \\
\hline & & & & & $n=$ & D - & & \\
\hline 6 & -3.353927000 & 1.786353000 & 0.186868000 & 6 & -1.353918000 & 2.528622000 & 0.883165000 & 6 \\
\hline 6 & -1.980324000 & 1.922648000 & -0.024305000 & 6 & -1.254904000 & 2.154798000 & -0.458691000 & 6 \\
\hline 6 & -3.931163000 & 0.532242000 & 0.325379000 & 6 & -1.047626000 & 1.607342000 & 1.877325000 & 6 \\
\hline 1 & -4.996999000 & 0.441238000 & 0.488046000 & 1 & -1.139381000 & 1.887282000 & 2.919418000 & 1 \\
\hline 6 & -1.193498000 & 0.772359000 & -0.095746000 & 6 & -0.835287000 & 0.866158000 & -0.783389000 & 6 \\
\hline 6 & -3.151051000 & -0.617722000 & 0.256156000 & 6 & -0.634103000 & 0.317177000 & 1.571937000 & 6 \\
\hline 1 & -3.593028000 & -1.597110000 & 0.362653000 & 1 & -0.437862000 & -0.399003000 & 2.356646000 & 1 \\
\hline 6 & -1.789604000 & -0.472773000 & 0.045238000 & 6 & -0.541099000 & -0.051000000 & 0.228242000 & 6 \\
\hline 35 & -0.695014000 & -2.049086000 & -0.055046000 & 35 & -1.399042000 & -2.106503000 & -0.182029000 & 35 \\
\hline 6 & 3.493303000 & 0.852015000 & 0.087385000 & 6 & 2.284605000 & 0.134979000 & 0.020855000 & 6 \\
\hline 1 & 3.720282000 & 1.916353000 & -0.019348000 & 1 & 2.082224000 & 0.937583000 & -0.689876000 & 1 \\
\hline 1 & 3.199099000 & 0.707490000 & 1.130212000 & 1 & 2.216733000 & 0.571099000 & 1.018718000 & 1 \\
\hline 6 & 4.754887000 & 0.033110000 & -0.202288000 & 6 & 3.672307000 & -0.459316000 & -0.216002000 & 6 \\
\hline 1 & 5.039054000 & 0.177448000 & -1.249446000 & 1 & 3.709865000 & -0.905319000 & -1.213724000 & 1 \\
\hline 1 & 4.520734000 & -1.030088000 & -0.091704000 & 1 & 3.842812000 & -1.273523000 & 0.493766000 & 1 \\
\hline 6 & 5.926769000 & 0.401978000 & 0.706476000 & 6 & 4.777482000 & 0.587108000 & -0.076413000 & 6 \\
\hline 1 & 6.198997000 & 1.453459000 & 0.593152000 & 1 & 4.646000000 & 1.398877000 & -0.793851000 & 1 \\
\hline 1 & 5.678366000 & 0.237897000 & 1.757081000 & 1 & 4.783446000 & 1.025025000 & 0.923149000 & 1 \\
\hline 1 & 6.811063000 & -0.194388000 & 0.479419000 & 1 & 5.758181000 & 0.145101000 & -0.251190000 & 1 \\
\hline 7 & 2.327448000 & 0.556829000 & -0.748831000 & 7 & 1.188269000 & -0.830983000 & -0.099293000 & 7 \\
\hline 1 & 2.051155000 & -0.413848000 & -0.648021000 & 1 & 1.239420000 & -1.594437000 & 0.567235000 & 1 \\
\hline 1 & 2.548203000 & 0.694092000 & -1.728731000 & 1 & 1.100234000 & -1.241928000 & -1.022972000 & 1 \\
\hline 1 & -0.126646000 & 0.854492000 & -0.261817000 & 1 & -0.790387000 & 0.561300000 & -1.820216000 & 1 \\
\hline 1 & -3.975946000 & 2.671066000 & 0.242469000 & 1 & -1.678725000 & 3.526365000 & 1.145346000 & 1 \\
\hline 6 & -1.352333000 & 3.285169000 & -0.166055000 & 6 & -1.640307000 & 3.115258000 & -1.554907000 & 6 \\
\hline 1 & -1.843394000 & 3.865814000 & -0.949203000 & 1 & -2.718129000 & 3.087231000 & -1.733503000 & 1 \\
\hline 1 & -1.441156000 & 3.855548000 & 0.761220000 & 1 & -1.381531000 & 4.140738000 & -1.289795000 & 1 \\
\hline 1 & -0.294777000 & 3.208462000 & -0.412205000 & 1 & -1.147202000 & 2.871337000 & -2.495822000 & 1 \\
\hline & & & & & $n=3$ & $D-O$ & & \\
\hline 6 & -2.737967000 & 1.784218000 & 0.120332000 & 6 & 2.318917000 & -0.914084000 & 1.164334000 & 6 \\
\hline 6 & -3.303562000 & 0.526838000 & -0.084865000 & 6 & 2.069064000 & -0.765884000 & -0.200332000 & 6 \\
\hline 6 & -1.360097000 & 1.892283000 & 0.289256000 & 6 & 1.431096000 & -0.322910000 & 2.061647000 & 6 \\
\hline 1 & -0.923716000 & 2.869581000 & 0.448315000 & 1 & 1.619650000 & -0.411840000 & 3.124230000 & 1 \\
\hline 6 & -2.487320000 & -0.608049000 & -0.119161000 & 6 & 0.957676000 & -0.052531000 & -0.653534000 & 6 \\
\hline 6 & -0.530214000 & 0.780605000 & 0.259081000 & 6 & 0.320057000 & 0.390636000 & 1.646933000 & 6 \\
\hline 1 & 0.539129000 & 0.875879000 & 0.391322000 & 1 & -0.325565000 & 0.874565000 & 2.364572000 & 1 \\
\hline 6 & -1.125035000 & -0.458711000 & 0.052604000 & 6 & 0.097216000 & 0.532963000 & 0.269841000 & 6 \\
\hline
\end{tabular}




\begin{tabular}{|c|c|c|c|c|c|c|c|c|}
\hline 35 & -0.022277000 & -2.029871000 & 0.001805000 & 35 & -0.498209000 & 2.635215000 & -0.292228000 & 35 \\
\hline 6 & 4.134434000 & 1.146642000 & -0.046399000 & 6 & -1.959914000 & -1.405776000 & 0.060877000 & 6 \\
\hline 1 & 3.855167000 & 1.187496000 & -1.102554000 & 1 & -1.222432000 & -1.941483000 & -0.538842000 & 1 \\
\hline 1 & 4.250560000 & 2.184920000 & 0.276659000 & 1 & -1.744050000 & -1.630974000 & 1.106208000 & 1 \\
\hline 6 & 5.474433000 & 0.418683000 & 0.097263000 & 6 & -3.376972000 & -1.842597000 & -0.306820000 & 6 \\
\hline 1 & 5.352039000 & -0.618579000 & -0.229354000 & 1 & -3.574647000 & -1.585297000 & -1.351157000 & 1 \\
\hline 1 & 5.743404000 & 0.376631000 & 1.157477000 & 1 & -4.097127000 & -1.278971000 & 0.292709000 & 1 \\
\hline 6 & 6.600878000 & 1.080725000 & -0.695251000 & 6 & -3.590326000 & -3.341230000 & -0.096068000 & 6 \\
\hline 1 & 6.370357000 & 1.106892000 & -1.762317000 & 1 & -2.904298000 & -3.928170000 & -0.709092000 & 1 \\
\hline 1 & 6.761957000 & 2.110169000 & -0.368302000 & 1 & -3.427018000 & -3.621438000 & 0.946044000 & 1 \\
\hline 1 & 7.542746000 & 0.544602000 & -0.574113000 & 1 & -4.606079000 & -3.632583000 & -0.362718000 & 1 \\
\hline 7 & 3.006344000 & 0.573366000 & 0.689999000 & 7 & -1.706792000 & 0.025716000 & -0.131915000 & 7 \\
\hline 1 & 3.211471000 & 0.529949000 & 1.681788000 & 1 & -2.271605000 & 0.626624000 & 0.459502000 & 1 \\
\hline 1 & 2.824996000 & -0.378157000 & 0.390198000 & 1 & -1.835050000 & 0.337402000 & -1.089312000 & 1 \\
\hline 1 & -2.938304000 & -1.575466000 & -0.279480000 & 1 & 0.826306000 & 0.080556000 & -1.717197000 & 1 \\
\hline 1 & -3.350053000 & 2.672159000 & 0.150162000 & 1 & 3.175968000 & -1.456433000 & 1.528607000 & 1 \\
\hline 8 & -4.632526000 & 0.294434000 & -0.262663000 & 8 & 2.859632000 & -1.279333000 & -1.185861000 & 8 \\
\hline 6 & -5.521947000 & 1.399460000 & -0.245713000 & 6 & 4.040107000 & -1.970504000 & -0.813659000 & 6 \\
\hline 1 & -5.500283000 & 1.916515000 & 0.717087000 & 1 & 4.719536000 & -1.327287000 & -0.248908000 & 1 \\
\hline 1 & -5.291725000 & 2.110765000 & -1.043039000 & 1 & 3.814789000 & -2.862150000 & -0.222448000 & 1 \\
\hline 1 & -6.513883000 & 0.986915000 & -0.408597000 & 1 & 4.517917000 & -2.268887000 & -1.743245000 & 1 \\
\hline & & & & & $n=$ & 3, $\mathbf{R}=$ & & \\
\hline 6 & -2.081095000 & 2.702365000 & -0.003272000 & 6 & -1.202040000 & 2.655066000 & 0.766138000 & 6 \\
\hline 6 & -0.863282000 & 2.045419000 & 0.002940000 & 6 & -1.094700000 & 2.188046000 & -0.532287000 & 6 \\
\hline 6 & -3.240027000 & 1.935857000 & -0.009240000 & 6 & -0.971654000 & 1.735254000 & 1.785307000 & 6 \\
\hline 9 & 0.268893000 & 2.779693000 & 0.009067000 & 9 & -1.325950000 & 3.042388000 & -1.553832000 & 9 \\
\hline 1 & -4.204865000 & 2.424407000 & -0.014359000 & 1 & -1.064212000 & 2.050249000 & 2.816730000 & 1 \\
\hline 6 & -0.749270000 & 0.664875000 & 0.004260000 & 6 & -0.770372000 & 0.883127000 & -0.853821000 & 6 \\
\hline 6 & -3.176528000 & 0.546817000 & -0.008847000 & 6 & -0.640975000 & 0.413662000 & 1.524881000 & 6 \\
\hline 1 & -4.077459000 & -0.047629000 & -0.015301000 & 1 & -0.511695000 & -0.286919000 & 2.336836000 & 1 \\
\hline 6 & -1.929507000 & -0.064353000 & -0.001824000 & 6 & -0.549292000 & -0.020157000 & 0.193444000 & 6 \\
\hline 35 & -1.834960000 & -1.977502000 & 0.000000000 & 35 & -1.535237000 & -1.995249000 & -0.148886000 & 35 \\
\hline 6 & 3.531165000 & 0.061382000 & 0.022096000 & 6 & 2.263850000 & 0.018800000 & 0.030983000 & 6 \\
\hline 1 & 3.436832000 & 0.737418000 & -0.831566000 & 1 & 2.114897000 & 0.802992000 & -0.712329000 & 1 \\
\hline 1 & 3.465096000 & 0.690157000 & 0.913733000 & 1 & 2.206317000 & 0.493722000 & 1.011354000 & 1 \\
\hline 6 & 4.898520000 & -0.626291000 & -0.018488000 & 6 & 3.616708000 & -0.663305000 & -0.164328000 & 6 \\
\hline 1 & 4.952387000 & -1.257712000 & -0.910978000 & 1 & 3.638462000 & -1.151426000 & -1.142603000 & 1 \\
\hline 1 & 4.982301000 & -1.300831000 & 0.839482000 & 1 & 3.734121000 & -1.455453000 & 0.580286000 & 1 \\
\hline 6 & 6.063822000 & 0.362232000 & -0.013902000 & 6 & 4.777846000 & 0.324658000 & -0.055587000 & 6 \\
\hline 1 & 6.019058000 & 1.029324000 & -0.877274000 & 1 & 4.700004000 & 1.109817000 & -0.809312000 & 1 \\
\hline 1 & 6.050361000 & 0.984606000 & 0.883198000 & 1 & 4.798549000 & 0.804895000 & 0.924090000 & 1 \\
\hline 1 & 7.024230000 & -0.153321000 & -0.044220000 & 1 & 5.733171000 & -0.179718000 & -0.198507000 & 1 \\
\hline 7 & 2.365427000 & -0.827480000 & 0.017495000 & 7 & 1.116450000 & -0.891603000 & -0.070750000 & 7 \\
\hline 1 & 2.389681000 & -1.452965000 & 0.814760000 & 1 & 1.120557000 & -1.630765000 & 0.625151000 & 1 \\
\hline 1 & 2.366360000 & -1.413925000 & -0.809609000 & 1 & 1.026712000 & -1.334283000 & -0.980043000 & 1 \\
\hline 1 & 0.229062000 & 0.196139000 & 0.008418000 & 1 & -0.753262000 & 0.576977000 & -1.888975000 & 1 \\
\hline 1 & -2.110291000 & 3.782192000 & -0.002708000 & 1 & -1.468140000 & 3.682451000 & 0.959687000 & 1 \\
\hline & & & & & $\boldsymbol{n}=$ & $\mathbf{P}$ & & \\
\hline 6 & -0.117720000 & 0.904157000 & -0.457194000 & 6 & -1.859963000 & 0.081707000 & 1.710991000 & 6 \\
\hline 6 & 0.373370000 & -0.376427000 & -0.210710000 & 6 & -1.792941000 & 0.072394000 & 0.321680000 & 6 \\
\hline 6 & 0.765453000 & 1.974051000 & -0.466502000 & 6 & -0.724487000 & -0.287397000 & 2.424576000 & 6 \\
\hline 1 & 0.396605000 & 2.972598000 & -0.657040000 & 1 & -0.754325000 & -0.311915000 & 3.506126000 & 1 \\
\hline 6 & 1.726491000 & -0.591763000 & 0.025029000 & 6 & -0.631966000 & -0.288474000 & -0.351678000 & 6 \\
\hline 6 & 2.123244000 & 1.780050000 & -0.233659000 & 6 & 0.451982000 & -0.645343000 & 1.785298000 & 6 \\
\hline 1 & 2.100249000 & -1.585213000 & 0.215522000 & 1 & -0.623267000 & -0.332373000 & -1.430453000 & 1 \\
\hline 1 & 2.808784000 & 2.614570000 & -0.241908000 & 1 & 1.308395000 & -0.966229000 & 2.361173000 & 1 \\
\hline 6 & 2.587707000 & 0.496287000 & 0.009813000 & 6 & 0.502382000 & -0.655059000 & 0.382962000 & 6 \\
\hline 35 & 4.450345000 & 0.213435000 & 0.330680000 & 35 & 1.518153000 & -2.466132000 & -0.428026000 & 35 \\
\hline 1 & -1.175260000 & 1.059375000 & -0.639503000 & 1 & -2.773247000 & 0.351392000 & 2.217145000 & 1 \\
\hline 6 & -4.447948000 & 0.957663000 & 0.192277000 & 6 & 1.843643000 & 1.795307000 & 0.069043000 & 6 \\
\hline 1 & -3.946593000 & 0.402052000 & 0.988397000 & 1 & 0.917226000 & 2.124348000 & -0.403509000 & 1 \\
\hline 1 & -4.498032000 & 1.996705000 & 0.529042000 & 1 & 1.706050000 & 1.897541000 & 1.146218000 & 1 \\
\hline 6 & -5.866966000 & 0.413433000 & 0.006767000 & 6 & 3.025622000 & 2.636058000 & -0.409702000 & 6 \\
\hline 1 & -5.805736000 & -0.624404000 & -0.334863000 & 1 & 3.154405000 & 2.499265000 & -1.486845000 & 1 \\
\hline 1 & -6.360845000 & 0.971984000 & -0.794769000 & 1 & 3.943320000 & 2.273176000 & 0.061443000 & 1 \\
\hline 6 & -6.705815000 & 0.488870000 & 1.281872000 & 6 & 2.835873000 & 4.120028000 & -0.096892000 & 6 \\
\hline 1 & -6.247295000 & -0.084876000 & 2.089798000 & 1 & 1.941948000 & 4.515068000 & -0.581990000 & 1 \\
\hline 1 & -6.808623000 & 1.519677000 & 1.627278000 & 1 & 2.733716000 & 4.288010000 & 0.976461000 & 1 \\
\hline 1 & -7.709146000 & 0.092367000 & 1.123054000 & 1 & 3.688122000 & 4.703040000 & -0.444850000 & 1 \\
\hline 7 & -3.583780000 & 0.911969000 & -0.991345000 & 7 & 1.981989000 & 0.359370000 & -0.210882000 & 7 \\
\hline 1 & -3.998807000 & 1.435761000 & -1.753747000 & 1 & 2.781610000 & -0.068751000 & 0.245926000 & 1 \\
\hline 1 & -3.483743000 & -0.042909000 & -1.317865000 & 1 & 2.051376000 & 0.143665000 & -1.200895000 & 1 \\
\hline 6 & -0.591986000 & -1.531267000 & -0.194856000 & 6 & -2.979282000 & 0.521814000 & -0.487888000 & 6 \\
\hline 9 & 0.017103000 & -2.715944000 & -0.006922000 & 9 & -3.075320000 & -0.122046000 & -1.668847000 & 9 \\
\hline 9 & -1.514097000 & -1.402190000 & 0.786169000 & 9 & -4.145118000 & 0.340278000 & 0.160638000 & 9 \\
\hline 9 & -1.283245000 & -1.623201000 & -1.355338000 & 9 & -2.902870000 & 1.845832000 & -0.780591000 & 9 \\
\hline & & & & & $n=3$ & $--C$ & ОH & \\
\hline 6 & -2.268165000 & 1.968267000 & 0.172651000 & 6 & 2.111105000 & -0.538779000 & 1.595332000 & 6 \\
\hline 6 & -2.963014000 & 0.774460000 & -0.021639000 & 6 & 2.010554000 & -0.488115000 & 0.204445000 & 6 \\
\hline 6 & -0.890090000 & 1.948640000 & 0.319724000 & 6 & 1.076130000 & -0.014442000 & 2.356376000 & 6 \\
\hline 1 & -0.347947000 & 2.872324000 & 0.470004000 & 1 & 1.139664000 & -0.026261000 & 3.436761000 & 1 \\
\hline 6 & -2.272401000 & -0.438170000 & -0.067745000 & 6 & 0.895440000 & 0.074215000 & -0.417468000 & 6 \\
\hline 6 & -0.186253000 & 0.748016000 & 0.276100000 & 6 & -0.046649000 & 0.548436000 & 1.763148000 & 6 \\
\hline 1 & 0.891163000 & 0.735035000 & 0.390464000 & 1 & -0.822810000 & 0.987249000 & 2.373989000 & 1 \\
\hline 6 & -0.895441000 & -0.429834000 & 0.082350000 & 6 & -0.133060000 & 0.602239000 & 0.366826000 & 6 \\
\hline 35 & 0.050968000 & -2.096228000 & 0.018651000 & 35 & -0.869486000 & 2.590953000 & -0.376062000 & 35 \\
\hline 6 & 4.391715000 & 1.033420000 & -0.071739000 & 6 & -1.920765000 & -1.567087000 & 0.052538000 & 6 \\
\hline 1 & 4.115552000 & 1.082933000 & -1.128259000 & 1 & -1.082940000 & -2.052615000 & -0.449999000 & 1 \\
\hline 1 & 4.450251000 & 2.068361000 & 0.275726000 & 1 & -1.776743000 & -1.712464000 & 1.124226000 & 1 \\
\hline 6 & 5.766640000 & 0.373050000 & 0.064718000 & 6 & -3.247986000 & -2.172517000 & -0.401461000 & 6 \\
\hline
\end{tabular}




\begin{tabular}{|c|c|c|c|c|c|c|c|c|}
\hline 1 & 5.699130000 & -0.662390000 & -0.283035000 & 1 & -3.376431000 & -1.999500000 & -1.473488000 & 1 \\
\hline 1 & 6.032975000 & 0.323743000 & 1.125228000 & 1 & -4.071745000 & -1.654377000 & 0.097298000 & 1 \\
\hline 6 & 6.860429000 & 1.108088000 & -0.708873000 & 6 & -3.325751000 & -3.670152000 & -0.106755000 & 6 \\
\hline 1 & 6.634252000 & 1.141960000 & -1.776590000 & 1 & -2.529628000 & -4.215446000 & -0.616240000 & 1 \\
\hline 1 & 6.965537000 & 2.138119000 & -0.361885000 & 1 & -3.233216000 & -3.868286000 & 0.962313000 & 1 \\
\hline 1 & 7.828321000 & 0.619757000 & -0.591779000 & 1 & -4.277366000 & -4.083589000 & -0.439822000 & 1 \\
\hline 7 & 3.291949000 & 0.382739000 & 0.646049000 & 7 & -1.800602000 & -0.127049000 & -0.205940000 & 7 \\
\hline 1 & 3.494107000 & 0.336143000 & 1.638560000 & 1 & -2.496350000 & 0.434398000 & 0.275104000 & 1 \\
\hline 1 & 3.177232000 & -0.574630000 & 0.331817000 & 1 & -1.846835000 & 0.116533000 & -1.190488000 & 1 \\
\hline 1 & -2.806886000 & -1.362449000 & -0.217908000 & 1 & 0.855552000 & 0.137422000 & -1.493469000 & 1 \\
\hline 1 & -2.823866000 & 2.893999000 & 0.204854000 & 1 & 2.988614000 & -0.972214000 & 2.048891000 & 1 \\
\hline 6 & -4.439681000 & 0.837078000 & -0.175497000 & 6 & 3.132691000 & -1.043480000 & -0.596732000 & 6 \\
\hline 8 & -5.093502000 & 1.850866000 & -0.145662000 & 8 & 4.134075000 & -1.537050000 & -0.137677000 & 8 \\
\hline 8 & -5.006684000 & -0.380234000 & -0.357748000 & 8 & 2.932650000 & -0.954858000 & -1.937138000 & 8 \\
\hline 1 & -5.958270000 & -0.227241000 & -0.445333000 & 1 & 3.722397000 & -1.331526000 & -2.350324000 & 1 \\
\hline & & & & & $n=$ & $\mathbf{R}=-$ & & \\
\hline 6 & -2.520566000 & 2.126748000 & 0.141439000 & 6 & 1.839088000 & -1.786919000 & 1.322694000 & 6 \\
\hline 6 & -3.290054000 & 0.977555000 & -0.063306000 & 6 & 1.717783000 & -1.598895000 & -0.057174000 & 6 \\
\hline 6 & -1.148043000 & 2.010556000 & 0.296783000 & 6 & 1.215749000 & -0.874505000 & 2.164319000 & 6 \\
\hline 1 & -0.547992000 & 2.896082000 & 0.455024000 & 1 & 1.309442000 & -0.983951000 & 3.236806000 & 1 \\
\hline 6 & -2.679968000 & -0.280560000 & -0.111012000 & 6 & 0.991017000 & -0.529975000 & -0.586792000 & 6 \\
\hline 6 & -0.524728000 & 0.767111000 & 0.251812000 & 6 & 0.483444000 & 0.193067000 & 1.668078000 & 6 \\
\hline 1 & 0.549117000 & 0.684543000 & 0.373591000 & 1 & 0.043545000 & 0.910356000 & 2.346401000 & 1 \\
\hline 6 & -1.306775000 & -0.362412000 & 0.047678000 & 6 & 0.371093000 & 0.378772000 & 0.279995000 & 6 \\
\hline 35 & -0.474710000 & -2.083870000 & -0.018807000 & 35 & 0.662704000 & 2.492451000 & -0.330183000 & 35 \\
\hline 6 & 4.016263000 & 0.939756000 & -0.054056000 & 6 & -2.228004000 & -0.670786000 & 0.025417000 & 6 \\
\hline 1 & 3.750707000 & 0.980639000 & -1.113603000 & 1 & -1.755535000 & -1.458254000 & -0.562985000 & 1 \\
\hline 1 & 4.058525000 & 1.976790000 & 0.289217000 & 1 & -2.111067000 & -0.941744000 & 1.075622000 & 1 \\
\hline 6 & 5.396738000 & 0.295709000 & 0.100697000 & 6 & -3.705148000 & -0.533673000 & -0.338135000 & 6 \\
\hline 1 & 5.344256000 & -0.742343000 & -0.241842000 & 1 & -3.794886000 & -0.244540000 & -1.388802000 & 1 \\
\hline 1 & 5.652508000 & 0.255382000 & 1.164141000 & 1 & -4.148010000 & 0.277436000 & 0.246476000 & 1 \\
\hline 6 & 6.490277000 & 1.038353000 & -0.665879000 & 6 & -4.477626000 & -1.829265000 & -0.091701000 & 6 \\
\hline 1 & 6.274771000 & 1.063811000 & -1.735997000 & 1 & -4.075433000 & -2.649418000 & -0.688433000 & 1 \\
\hline 1 & 6.580921000 & 2.071265000 & -0.323518000 & 1 & -4.428841000 & -2.125519000 & 0.957398000 & 1 \\
\hline 1 & 7.461919000 & 0.560973000 & -0.536068000 & 1 & -5.528274000 & -1.711148000 & -0.355069000 & 1 \\
\hline 7 & 2.916414000 & 0.278706000 & 0.655958000 & 7 & -1.446713000 & 0.555438000 & -0.190745000 & 7 \\
\hline 1 & 3.109289000 & 0.242700000 & 1.650900000 & 1 & -1.771687000 & 1.344753000 & 0.359822000 & 1 \\
\hline 1 & 2.823859000 & -0.683485000 & 0.348873000 & 1 & -1.426626000 & 0.856306000 & -1.160728000 & 1 \\
\hline 1 & -3.275322000 & -1.166715000 & -0.269111000 & 1 & 0.952736000 & -0.386829000 & -1.656321000 & 1 \\
\hline 1 & -3.002209000 & 3.093058000 & 0.176605000 & 1 & 2.411832000 & -2.613698000 & 1.712817000 & 1 \\
\hline 6 & -4.706694000 & 1.081760000 & -0.225141000 & 6 & 2.348421000 & -2.513699000 & -0.958744000 & 6 \\
\hline 7 & -5.848441000 & 1.169192000 & -0.355110000 & 7 & 2.845750000 & -3.257475000 & -1.685591000 & 7 \\
\hline & & & & & $n=$ & , K & & \\
\hline 6 & -3.111412000 & 2.448779000 & -0.017415000 & 6 & -0.903514000 & 3.104103000 & -0.000333000 & 6 \\
\hline 6 & -1.729737000 & 2.320074000 & 0.061299000 & 6 & -0.829958000 & 2.397196000 & -1.196097000 & 6 \\
\hline 6 & -3.907690000 & 1.311919000 & -0.092924000 & 6 & -0.830860000 & 2.397454000 & 1.195646000 & 6 \\
\hline 1 & -1.103871000 & 3.200674000 & 0.120047000 & 1 & -0.903927000 & 2.919398000 & -2.141778000 & 1 \\
\hline 1 & -4.983972000 & 1.402794000 & -0.154316000 & 1 & -0.905522000 & 2.919865000 & 2.141157000 & 1 \\
\hline 6 & -1.131338000 & 1.063411000 & 0.065547000 & 6 & -0.678824000 & 1.016180000 & -1.212818000 & 6 \\
\hline 6 & -3.329033000 & 0.047353000 & -0.090030000 & 6 & -0.679741000 & 1.016448000 & 1.212787000 & 6 \\
\hline 1 & -0.055042000 & 0.962574000 & 0.126106000 & 1 & -0.664341000 & 0.479770000 & -2.150880000 & 1 \\
\hline 1 & -3.941733000 & -0.840190000 & -0.148563000 & 1 & -0.665913000 & 0.480241000 & 2.150973000 & 1 \\
\hline 6 & -1.947473000 & -0.055985000 & -0.010599000 & 6 & -0.613909000 & 0.324068000 & 0.000085000 & 6 \\
\hline 35 & -1.149293000 & -1.802573000 & -0.006825000 & 35 & -1.861471000 & -1.563843000 & -0.000142000 & 35 \\
\hline 1 & -3.566216000 & 3.430072000 & -0.019959000 & 1 & -1.027686000 & 4.177233000 & -0.000495000 & 1 \\
\hline 6 & 3.631853000 & 0.824824000 & 0.001454000 & 6 & 2.191795000 & -0.083250000 & 0.001510000 & 6 \\
\hline 1 & 3.591731000 & 1.303587000 & -0.980673000 & 1 & 2.181114000 & 0.568269000 & -0.873514000 & 1 \\
\hline 1 & 3.779013000 & 1.630646000 & 0.725645000 & 1 & 2.181973000 & 0.564833000 & 0.879070000 & 1 \\
\hline 6 & 4.829466000 & -0.128711000 & 0.052932000 & 6 & 3.434960000 & -0.971709000 & -0.000861000 & 6 \\
\hline 1 & 4.6755890000 & -0.931294000 & -0.675033000 & 1 & 3.411769000 & -1.624267000 & -0.878005000 & 1 \\
\hline 1 & 4.859212000 & -0.608450000 & 1.036271000 & 1 & 3.412773000 & -1.627633000 & 0.873788000 & 1 \\
\hline 6 & 6.160488000 & 0.570195000 & -0.221110000 & 6 & 4.725619000 & -0.152996000 & -0.000045000 & 6 \\
\hline 1 & 6.167543000 & 1.033205000 & -1.210061000 & 1 & 4.786587000 & 0.489492000 & -0.880043000 & 1 \\
\hline 1 & 6.351457000 & 1.356800000 & 0.511824000 & 1 & 4.787789000 & 0.485854000 & 0.882514000 & 1 \\
\hline 1 & 6.995087000 & -0.130374000 & -0.178718000 & 1 & 5.600382000 & -0.802925000 & -0.001981000 & 1 \\
\hline 7 & 2.320361000 & 0.226517000 & 0.259724000 & 7 & 0.925397000 & -0.822972000 & 0.000624000 & 7 \\
\hline 1 & 2.297466000 & -0.202546000 & 1.178019000 & 1 & 0.792234000 & -1.410264000 & 0.817592000 & 1 \\
\hline 1 & 2.125703000 & -0.514233000 & -0.404683000 & 1 & 0.793058000 & -1.409510000 & -0.817026000 & 1 \\
\hline & & & & & $n=$ & $\mathbf{R}=-$ & & \\
\hline 6 & -3.232672000 & -1.636480000 & -0.055423000 & 6 & -3.087301000 & $\begin{array}{l}-0.994396000 \\
\end{array}$ & $\begin{array}{l}-0.077922000 \\
\end{array}$ & 6 \\
\hline 6 & -1.860311000 & -1.774076000 & 0.152376000 & 6 & -2.703201000 & -0.315929000 & 1.079979000 & 6 \\
\hline 6 & -3.739044000 & -0.351282000 & -0.249822000 & 6 & -2.146271000 & -1.079231000 & -1.102138000 & 6 \\
\hline 1 & -1.438428000 & -2.760755000 & 0.299407000 & 1 & -3.405034000 & -0.215588000 & 1.899863000 & 1 \\
\hline 1 & -4.799587000 & -0.212987000 & -0.420286000 & 1 & -2.405235000 & -1.584499000 & -2.025330000 & 1 \\
\hline 6 & -1.008602000 & -0.674671000 & 0.173516000 & 6 & -1.445066000 & 0.254337000 & 1.221952000 & 6 \\
\hline 6 & -2.909481000 & 0.763445000 & -0.233383000 & 6 & -0.875798000 & -0.521493000 & -0.995672000 & 6 \\
\hline 1 & 0.054125000 & -0.805336000 & 0.334718000 & 1 & -1.193620000 & 0.801896000 & 2.119176000 & 1 \\
\hline 1 & -3.316109000 & 1.752156000 & -0.388375000 & 1 & -0.190642000 & -0.574174000 & -1.828264000 & 1 \\
\hline 6 & -1.550762000 & 0.586615000 & -0.019933000 & 6 & -0.534413000 & 0.159239000 & 0.168946000 & 6 \\
\hline 35 & -0.403065000 & 2.126257000 & -0.001868000 & 35 & 0.538245000 & 2.148172000 & -0.238307000 & 35 \\
\hline 6 & -4.144592000 & -2.835320000 & -0.045590000 & 6 & -4.471935000 & -1.570149000 & -0.227372000 & 6 \\
\hline 1 & -5.005248000 & -2.686141000 & -0.697649000 & 1 & -4.492834000 & -2.364482000 & -0.973990000 & 1 \\
\hline 1 & -3.622922000 & -3.734374000 & -0.373220000 & 1 & -4.835454000 & -1.987815000 & 0.712823000 & 1 \\
\hline 1 & -4.527744000 & -3.029047000 & 0.959741000 & 1 & -5.192653000 & -0.810171000 & -0.542160000 & 1 \\
\hline 6 & 3.635217000 & -1.144599000 & -0.024284000 & 6 & 2.110916000 & -1.024417000 & -0.060264000 & 6 \\
\hline 1 & 3.725432000 & -2.196241000 & 0.261472000 & 1 & 1.611447000 & -1.826690000 & -0.605683000 & 1 \\
\hline 1 & 3.373279000 & -1.140254000 & -1.085620000 & 1 & 2.327068000 & -0.224013000 & -0.767712000 & 1 \\
\hline 6 & 4.986404000 & -0.449383000 & 0.168459000 & 6 & 3.399616000 & -1.538050000 & 0.581421000 & 6 \\
\hline 1 & 5.238410000 & -0.452684000 & 1.233637000 & 1 & 3.156425000 & -2.308875000 & 1.318486000 & 1 \\
\hline 1 & 4.889165000 & 0.601619000 & -0.120442000 & 1 & 3.878217000 & -0.721844000 & 1.128922000 & 1 \\
\hline
\end{tabular}




\begin{tabular}{|c|c|c|c|c|c|c|c|c|}
\hline 6 & 6.112889000 & -1.103219000 & -0.630755000 & 6 & 4.370321000 & -2.103191000 & -0.455311000 & 6 \\
\hline 1 & 6.249048000 & -2.147107000 & -0.340341000 & 1 & 3.927163000 & -2.940801000 & -0.996821000 & 1 \\
\hline 1 & 5.899283000 & -1.084962000 & -1.701490000 & 1 & 4.650590000 & -1.344149000 & -1.187234000 & 1 \\
\hline 1 & 7.062635000 & -0.590514000 & -0.474830000 & 1 & 5.283920000 & -2.460814000 & 0.019206000 & 1 \\
\hline 7 & 2.506284000 & -0.575982000 & 0.714968000 & 7 & 1.142910000 & -0.483568000 & 0.901642000 & 7 \\
\hline 1 & 2.353270000 & 0.391323000 & 0.451823000 & 1 & 1.498819000 & 0.339278000 & 1.378153000 & 1 \\
\hline 1 & 2.694404000 & -0.578003000 & 1.711106000 & 1 & 0.841656000 & -1.178623000 & 1.578106000 & 1 \\
\hline & & & & & $n=4$, & $\mathbf{R}=$ & $1 \mathrm{e}$ & \\
\hline 6 & 2.929815000 & 1.297110000 & -0.004099000 & 6 & 2.561264000 & 0.501450000 & 0.280679000 & 6 \\
\hline 6 & 1.545721000 & 1.486782000 & -0.033493000 & 6 & 1.815526000 & 0.193069000 & 1.419316000 & 6 \\
\hline 6 & 3.446106000 & 0.002945000 & 0.028639000 & 6 & 1.992705000 & 0.287680000 & -0.970625000 & 6 \\
\hline 1 & 1.160667000 & 2.496696000 & -0.058602000 & 1 & 2.264164000 & 0.341821000 & 2.392257000 & 1 \\
\hline 1 & 4.510234000 & -0.174812000 & 0.051512000 & 1 & 2.542081000 & 0.495413000 & -1.876793000 & 1 \\
\hline 6 & 0.682302000 & 0.404490000 & -0.030256000 & 6 & 0.530534000 & -0.314990000 & 1.318907000 & 6 \\
\hline 6 & 2.580094000 & -1.088651000 & 0.032099000 & 6 & 0.697810000 & -0.222576000 & -1.087130000 & 6 \\
\hline 1 & 2.980725000 & -2.091544000 & 0.057507000 & 1 & 0.289445000 & -0.418384000 & -2.068106000 & 1 \\
\hline 6 & 1.213159000 & -0.880733000 & 0.002826000 & 6 & -0.022128000 & -0.537249000 & 0.056723000 & 6 \\
\hline 35 & 0.032618000 & -2.394098000 & 0.008128000 & 35 & -1.113376000 & -2.552988000 & -0.088646000 & 35 \\
\hline 6 & -3.987800000 & 1.338200000 & 0.015178000 & 6 & -1.784508000 & 1.723775000 & 0.007754000 & 6 \\
\hline 1 & -3.865641000 & 1.872697000 & 0.961112000 & 1 & -1.276158000 & 2.018549000 & 0.927211000 & 1 \\
\hline 1 & -3.891559000 & 2.090461000 & -0.772428000 & 1 & -1.157493000 & 2.057140000 & -0.820938000 & 1 \\
\hline 6 & -5.388448000 & 0.720856000 & -0.041696000 & 6 & -3.168519000 & 2.367381000 & -0.072855000 & 6 \\
\hline 1 & -5.477313000 & -0.031761000 & 0.747878000 & 1 & -3.782913000 & 2.008029000 & 0.757213000 & 1 \\
\hline 1 & -5.502354000 & 0.185631000 & -0.989581000 & 1 & -3.665656000 & 2.039985000 & -0.990109000 & 1 \\
\hline 6 & -6.502510000 & 1.756816000 & 0.103561000 & 6 & -3.097175000 & 3.893748000 & -0.040108000 & 6 \\
\hline 1 & -6.428335000 & 2.285393000 & 1.056233000 & 1 & -2.628362000 & 4.247821000 & 0.879622000 & 1 \\
\hline 1 & -6.453113000 & 2.503535000 & -0.691695000 & 1 & -2.516050000 & 4.280537000 & -0.879064000 & 1 \\
\hline 1 & -7.488035000 & 1.292080000 & 0.059568000 & 1 & -4.092973000 & 4.333117000 & -0.095668000 & 1 \\
\hline 7 & -2.866139000 & 0.406339000 & -0.118186000 & 7 & -1.799511000 & 0.259345000 & -0.026594000 & 7 \\
\hline 1 & -2.921438000 & -0.098601000 & -0.995574000 & 1 & -2.190302000 & -0.131114000 & -0.877573000 & 1 \\
\hline 1 & -2.890394000 & -0.293743000 & 0.614678000 & 1 & -2.288632000 & -0.168580000 & 0.752555000 & 1 \\
\hline 1 & -0.388887000 & 0.561466000 & -0.053035000 & 1 & -0.013441000 & -0.583104000 & 2.213195000 & 1 \\
\hline 8 & 3.685735000 & 2.429687000 & -0.010053000 & 8 & 3.822352000 & 0.994608000 & 0.499024000 & 8 \\
\hline 6 & 5.097511000 & 2.302384000 & 0.019668000 & 6 & 4.629754000 & 1.274347000 & -0.627245000 & 6 \\
\hline 1 & 5.435098000 & 1.794585000 & 0.927086000 & 1 & 4.805583000 & 0.378254000 & -1.230087000 & 1 \\
\hline 1 & 5.469873000 & 1.762755000 & -0.855202000 & 1 & 4.186163000 & 2.048505000 & -1.261369000 & 1 \\
\hline 1 & 5.490215000 & 3.315516000 & 0.009236000 & 1 & 5.578899000 & 1.634491000 & -0.238281000 & 1 \\
\hline & & & & & $n=$ & 4, $\mathrm{R}=$ & & \\
\hline 6 & 2.872448000 & 1.987414000 & -0.000067000 & 6 & -1.823967000 & -2.258596000 & -0.000002000 & 6 \\
\hline 6 & 1.491508000 & 1.907428000 & 0.000789000 & 6 & -1.501455000 & -1.660157000 & 1.203964000 & 6 \\
\hline 6 & 3.674736000 & 0.860230000 & -0.000880000 & 6 & -1.501482000 & -1.660146000 & -1.203968000 & 6 \\
\hline 1 & 0.900243000 & 2.812035000 & 0.001412000 & 1 & -1.781709000 & -2.135933000 & 2.133573000 & 1 \\
\hline 1 & 4.750260000 & 0.963964000 & -0.001530000 & 1 & -1.781760000 & -2.135911000 & -2.133575000 & 1 \\
\hline 6 & 0.884437000 & 0.655900000 & 0.000808000 & 6 & -0.834065000 & -0.440138000 & 1.211265000 & 6 \\
\hline 6 & 3.070414000 & -0.391573000 & -0.000841000 & 6 & -0.834090000 & -0.440127000 & -1.211272000 & 6 \\
\hline 1 & -0.195808000 & 0.575196000 & 0.001486000 & 1 & -0.616252000 & 0.047130000 & 2.150554000 & 1 \\
\hline 1 & 3.675260000 & -1.286138000 & -0.001462000 & 1 & -0.616311000 & 0.047156000 & -2.150560000 & 1 \\
\hline 6 & 1.685024000 & -0.478214000 & 0.000008000 & 6 & -0.510261000 & 0.176193000 & -0.000004000 & 6 \\
\hline 35 & 0.863309000 & -2.210475000 & 0.000056000 & 35 & -0.951701000 & 2.404819000 & 0.000010000 & 35 \\
\hline 9 & 3.457516000 & 3.203180000 & -0.000104000 & 9 & -2.476331000 & -3.447857000 & -0.000020000 & 9 \\
\hline 6 & -3.790520000 & 0.895957000 & 0.000523000 & 6 & 2.247800000 & -0.495790000 & 0.000115000 & 6 \\
\hline 1 & -3.740756000 & 1.550870000 & 0.874494000 & 1 & 1.995852000 & -1.094517000 & 0.876718000 & 1 \\
\hline 1 & -3.739639000 & 1.551369000 & -0.873010000 & 1 & 1.995623000 & -1.094861000 & -0.876191000 & 1 \\
\hline 6 & -5.128233000 & 0.150407000 & -0.000522000 & 6 & 3.733482000 & -0.137889000 & -0.000153000 & 6 \\
\hline 1 & -5.169736000 & -0.505269000 & 0.874844000 & 1 & 3.957738000 & 0.477150000 & 0.875644000 & 1 \\
\hline 1 & -5.168679000 & -0.504735000 & -0.876337000 & 1 & 3.957521000 & 0.476703000 & -0.876319000 & 1 \\
\hline 6 & -6.333846000 & 1.089280000 & -0.000969000 & 6 & 4.622413000 & -1.381322000 & 0.000048000 & 6 \\
\hline 1 & -6.332168000 & 1.734544000 & 0.879956000 & 1 & 4.439123000 & -1.997734000 & 0.881615000 & 1 \\
\hline 1 & -6.331231000 & 1.734909000 & -0.881624000 & 1 & 4.438893000 & -1.998182000 & -0.881157000 & 1 \\
\hline 1 & -7.272080000 & 0.533663000 & -0.001580000 & 1 & 5.677007000 & -1.106885000 & -0.000159000 & 1 \\
\hline 7 & -2.587662000 & 0.059251000 & 0.001051000 & 7 & 1.349828000 & 0.664084000 & -0.000012000 & 7 \\
\hline 1 & -2.575168000 & -0.548915000 & -0.810153000 & 1 & 1.445873000 & 1.258472000 & -0.817157000 & 1 \\
\hline 1 & -2.576109000 & -0.549208000 & 0.812042000 & 1 & 1.445853000 & 1.258697000 & 0.816970000 & 1 \\
\hline & & & & & $n$ & & & \\
\hline 6 & 2.541088000 & -0.510892000 & -0.018317000 & 6 & 2.045256000 & -0.092242000 & -0.000045000 & 6 \\
\hline 6 & 1.220782000 & -0.879753000 & -0.253215000 & 6 & 1.346248000 & -0.228317000 & 1.198854000 & 6 \\
\hline 6 & 2.857703000 & 0.820210000 & 0.235579000 & 6 & 1.346065000 & -0.227849000 & -1.198880000 & 6 \\
\hline 1 & 0.975001000 & -1.912373000 & -0.454671000 & 1 & 1.872512000 & -0.153181000 & 2.140503000 & 1 \\
\hline 1 & 3.883355000 & 1.110163000 & 0.412895000 & 1 & 1.872184000 & -0.152350000 & -2.140578000 & 1 \\
\hline 6 & 0.209087000 & 0.072841000 & -0.240171000 & 6 & -0.012353000 & -0.484952000 & 1.213398000 & 6 \\
\hline 6 & 1.857552000 & 1.782024000 & 0.252118000 & 6 & -0.012547000 & -0.484479000 & -1.213307000 & 6 \\
\hline 1 & 2.097147000 & 2.817055000 & 0.444603000 & 1 & -0.524015000 & -0.634892000 & -2.153237000 & 1 \\
\hline 6 & 0.545482000 & 1.394570000 & 0.014689000 & 6 & -0.711664000 & -0.617705000 & 0.000073000 & 6 \\
\hline 35 & -0.829896000 & 2.725448000 & 0.037305000 & 35 & -2.115080000 & -2.317637000 & -0.000124000 & 35 \\
\hline 6 & -3.981744000 & -1.585842000 & 0.002388000 & 6 & -1.802936000 & 1.963635000 & 0.000588000 & 6 \\
\hline 1 & -3.665713000 & -2.602108000 & -0.247562000 & 1 & -1.175221000 & 2.123326000 & 0.877985000 & 1 \\
\hline 1 & -3.717438000 & -1.435163000 & 1.052334000 & 1 & -1.174045000 & 2.123241000 & -0.875989000 & 1 \\
\hline 6 & -5.499486000 & -1.469833000 & -0.162608000 & 6 & -2.991756000 & 2.923010000 & -0.000269000 & 6 \\
\hline 1 & -5.754762000 & -1.619153000 & -1.216496000 & 1 & -3.616279000 & 2.726614000 & 0.875567000 & 1 \\
\hline 1 & -5.806456000 & -0.449079000 & 0.085571000 & 1 & -3.615079000 & 2.726482000 & -0.876932000 & 1 \\
\hline 6 & -6.271180000 & -2.466141000 & 0.701625000 & 6 & -2.546136000 & 4.384963000 & -0.000081000 & 6 \\
\hline 1 & -6.004162000 & -3.495216000 & 0.452532000 & 1 & -1.945944000 & 4.615198000 & 0.881573000 & 1 \\
\hline 1 & -6.057046000 & -2.317960000 & 1.761997000 & 1 & -1.944656000 & 4.615027000 & -0.880903000 & 1 \\
\hline 1 & -7.347895000 & -2.362048000 & 0.564475000 & 1 & -3.406155000 & 5.054000000 & -0.000773000 & 1 \\
\hline 7 & -3.175491000 & -0.658230000 & -0.797043000 & 7 & -2.186136000 & 0.544142000 & 0.000362000 & 7 \\
\hline 1 & -3.414920000 & 0.302154000 & -0.575950000 & 1 & -2.724574000 & 0.272331000 & -0.816886000 & 1 \\
\hline 1 & -3.368252000 & -0.781618000 & -1.784845000 & 1 & -2.724589000 & 0.272036000 & 0.817499000 & 1 \\
\hline 1 & -0.818120000 & -0.216581000 & -0.426875000 & 1 & -0.523682000 & -0.635732000 & 2.153342000 & 1 \\
\hline 6 & 3.623111000 & -1.554318000 & 0.015037000 & 6 & 3.497974000 & 0.245400000 & -0.000053000 & 6 \\
\hline 9 & 3.831747000 & -2.018627000 & 1.268945000 & 9 & 3.720486000 & 1.589046000 & 0.000824000 & 9 \\
\hline
\end{tabular}




\begin{tabular}{|c|c|c|c|c|c|c|c|c|c|c|c|}
\hline $\begin{array}{l}9 \\
9 \\
\end{array}$ & $\begin{array}{l}3.324119000 \\
4.806320000\end{array}$ & $\begin{array}{l}-2.624057000 \\
-1.072687000\end{array}$ & $\begin{array}{l}-0.748246000 \\
-0.418159000\end{array}$ & $\begin{array}{l}9 \\
9 \\
\end{array}$ & $\begin{array}{l}4.140562000 \\
4.140220000\end{array}$ & $\begin{array}{l}-0.235949000 \\
-0.234497000\end{array}$ & $\begin{array}{r}1.086907000 \\
-1.087848000\end{array}$ & $\begin{array}{l}9 \\
9\end{array}$ & $\begin{array}{l}-4.480923000 \\
-4.609570000\end{array}$ & $\begin{array}{l}-0.192627000 \\
-1.107469000\end{array}$ & $\begin{array}{r}-1.441768000 \\
0.524503000 \\
\end{array}$ \\
\hline \multicolumn{12}{|c|}{$n=4, \mathrm{R}=-\mathrm{COOH}$} \\
\hline 6 & 2.760494000 & -0.888603000 & 0.023735000 & 6 & -2.318128000 & 0.054149000 & 0.037425000 & 6 & -3.010866000 & 0.159480000 & 0.053337000 \\
\hline 6 & 1.408364000 & -1.124727000 & -0.228716000 & 6 & -1.634716000 & -0.125713000 & -1.172131000 & 6 & -2.233443000 & 0.740426000 & -0.947772000 \\
\hline 6 & 3.197479000 & 0.413421000 & 0.272267000 & 6 & -1.602151000 & -0.117133000 & 1.229570000 & 6 & -2.397319000 & -0.216088000 & 1.252385000 \\
\hline 1 & 1.066440000 & -2.130584000 & -0.421387000 & 1 & -2.169969000 & -0.023554000 & -2.105280000 & 1 & -2.695170000 & 1.032999000 & -1.878948000 \\
\hline 1 & 4.246546000 & 0.584715000 & 0.466478000 & 1 & -2.127504000 & -0.003529000 & 2.168043000 & 1 & -3.002188000 & -0.666589000 & 2.026183000 \\
\hline 6 & 0.494969000 & -0.078648000 & -0.234535000 & 6 & -0.293522000 & -0.447759000 & -1.202934000 & 6 & -0.875597000 & 0.948005000 & -0.762273000 \\
\hline 6 & 2.298792000 & 1.467784000 & 0.269512000 & 6 & -0.263075000 & -0.440815000 & 1.230669000 & 6 & -1.046495000 & -0.015115000 & 1.442892000 \\
\hline 1 & 2.632882000 & 2.476549000 & 0.461391000 & 1 & 0.256081000 & -0.610898000 & 2.163356000 & 1 & -0.581346000 & -0.316704000 & 2.373212000 \\
\hline 6 & 0.957359000 & 1.206236000 & 0.015530000 & 6 & 0.416498000 & -0.611458000 & 0.005128000 & 6 & -0.264864000 & 0.572748000 & 0.438145000 \\
\hline 35 & -0.282078000 & 2.664094000 & 0.012063000 & 35 & 1.728351000 & -2.354041000 & -0.007406000 & 35 & 2.555183000 & -2.094731000 & -0.274887000 \\
\hline 6 & -3.819668000 & -1.377051000 & 0.026781000 & 6 & 1.583895000 & 1.928095000 & -0.007406000 & 6 & 1.882253000 & 1.716480000 & -0.113251000 \\
\hline 1 & -3.562546000 & -2.418786000 & -0.183104000 & 1 & 0.955233000 & 2.110267000 & -0.879498000 & 1 & 1.984468000 & 1.366606000 & -1.142522000 \\
\hline 1 & -3.549901000 & -1.202546000 & 1.071643000 & 1 & 0.967954000 & 2.105657000 & 0.874665000 & 1 & 1.342487000 & 2.669487000 & -0.144659000 \\
\hline 6 & -5.327694000 & -1.179862000 & -0.149692000 & 6 & 2.806010000 & 2.844090000 & -0.013705000 & 6 & 3.265042000 & 1.916403000 & 0.496073000 \\
\hline 1 & -5.588410000 & -1.353750000 & -1.198496000 & 1 & 3.416355000 & 2.628303000 & -0.895008000 & 1 & 3.782392000 & 0.955722000 & 0.534371000 \\
\hline 1 & -5.575951000 & -0.134493000 & 0.058652000 & 1 & 3.428845000 & 2.623037000 & 0.857488000 & 1 & 3.154366000 & 2.258408000 & 1.530206000 \\
\hline 6 & -6.158277000 & -2.096587000 & 0.747446000 & 6 & 2.413513000 & 4.321234000 & -0.006242000 & 6 & 4.101276000 & 2.925267000 & -0.288094000 \\
\hline 1 & -5.949685000 & -3.148055000 & 0.539408000 & 1 & 1.814908000 & 4.575754000 & -0.882273000 & 1 & 4.262236000 & 2.590394000 & -1.314043000 \\
\hline 1 & -5.939789000 & -1.920237000 & 1.802633000 & 1 & 1.828239000 & 4.570348000 & 0.880244000 & 1 & 3.614998000 & 3.901690000 & -0.327422000 \\
\hline 1 & -7.226798000 & -1.936559000 & 0.600291000 & 1 & 3.297283000 & 4.958574000 & -0.011002000 & 1 & 5.079589000 & 3.060510000 & 0.172306000 \\
\hline 7 & -2.958077000 & -0.529475000 & -0.802341000 & 7 & 1.919521000 & 0.495734000 & -0.013375000 & 7 & 1.116146000 & 0.717789000 & 0.644992000 \\
\hline 1 & -3.134731000 & 0.451111000 & -0.613857000 & 1 & 2.459545000 & 0.207537000 & 0.797407000 & 1 & 1.346930000 & 0.735014000 & 1.629523000 \\
\hline 1 & -3.157237000 & -0.674055000 & -1.785895000 & 1 & 2.439610000 & 0.209233000 & -0.837623000 & 1 & 1.875799000 & -0.856729000 & 0.169432000 \\
\hline 1 & -0.554018000 & -0.266309000 & -0.428849000 & 1 & 0.201873000 & -0.623751000 & -2.147325000 & 1 & -0.295090000 & 1.396045000 & -1.554417000 \\
\hline 6 & 3.766969000 & -1.978225000 & 0.039587000 & 6 & -3.740734000 & 0.395007000 & 0.108062000 & 6 & -4.461871000 & -0.076469000 & -0.098730000 \\
\hline 8 & 4.946083000 & -1.828243000 & 0.251812000 & 8 & -4.377482000 & 0.567332000 & 1.125092000 & 8 & -5.177741000 & -0.568974000 & 0.741122000 \\
\hline 8 & 3.232922000 & -3.199065000 & -0.212810000 & 8 & -4.328642000 & 0.515414000 & -1.117588000 & 8 & -4.943817000 & 0.321181000 & -1.304687000 \\
\hline 1 & 3.967695000 & -3.827911000 & -0.178225000 & 1 & -5.253932000 & 0.734163000 & -0.941001000 & 1 & -5.890136000 & 0.119713000 & -1.294891000 \\
\hline \multicolumn{12}{|c|}{$n=4, \mathrm{R}=-\mathrm{CN}$} \\
\hline 6 & -2.699217000 & -1.695331000 & 0.000631000 & 6 & -2.601365000 & 0.535953000 & -0.000015000 & 6 & -3.403586000 & 0.139598000 & -0.043356000 \\
\hline 6 & -3.489100000 & -0.542537000 & 0.056891000 & 6 & -1.942097000 & 0.243154000 & -1.203117000 & 6 & -2.614432000 & 0.721363000 & -1.038401000 \\
\hline 6 & -1.306527000 & -1.579579000 & -0.052066000 & 6 & -1.942385000 & 0.242652000 & 1.203118000 & 6 & -2.806835000 & -0.219389000 & 1.172432000 \\
\hline 1 & -4.565364000 & -0.629980000 & 0.097529000 & 1 & -2.441461000 & 0.434859000 & -2.143164000 & 1 & -3.064988000 & 1.000105000 & -1.980307000 \\
\hline 1 & -0.696403000 & -2.470574000 & -0.095370000 & 1 & -2.441973000 & 0.433957000 & 2.143128000 & 1 & -3.407029000 & -0.671393000 & 1.949060000 \\
\hline 6 & -2.893141000 & 0.708535000 & 0.060276000 & 6 & -0.675604000 & -0.301709000 & -1.215424000 & 6 & -1.264043000 & 0.945588000 & -0.829687000 \\
\hline 6 & -0.702154000 & -0.330961000 & -0.049007000 & 6 & -0.675892000 & -0.302217000 & 1.215507000 & 6 & -1.461237000 & 0.001508000 & 1.380078000 \\
\hline 1 & 0.377822000 & -0.247115000 & -0.089434000 & 1 & -0.208000000 & -0.559350000 & 2.155100000 & 1 & -1.010610000 & -0.287107000 & 2.321237000 \\
\hline 6 & -1.507623000 & 0.799257000 & 0.007229000 & 6 & -0.014649000 & -0.581021000 & 0.000070000 & 6 & -0.668058000 & 0.589529000 & 0.384753000 \\
\hline 35 & -0.695105000 & 2.528647000 & 0.012325000 & 35 & 0.985460000 & -2.516246000 & -0.000151000 & 35 & 2.164424000 & -2.116884000 & -0.205629000 \\
\hline 6 & 3.806671000 & -0.964168000 & -0.001525000 & 6 & 1.549301000 & 1.730707000 & 0.000355000 & 6 & 1.479888000 & 1.734309000 & -0.158028000 \\
\hline 1 & 3.620421000 & -1.472306000 & 0.948206000 & 1 & 0.964960000 & 2.010614000 & -0.876766000 & 1 & 1.595723000 & 1.360871000 & -1.177595000 \\
\hline 1 & 3.664289000 & -1.716249000 & -0.781978000 & 1 & 0.965204000 & 2.010652000 & 0.877617000 & 1 & 0.940969000 & 2.686587000 & -0.218515000 \\
\hline 6 & 5.252833000 & -0.462658000 & -0.035422000 & 6 & 2.904101000 & 2.434885000 & 0.000123000 & 6 & 2.854620000 & 1.949277000 & 0.464248000 \\
\hline 1 & 5.385460000 & 0.291501000 & 0.746587000 & 1 & 3.477426000 & 2.119772000 & -0.876161000 & 1 & 3.373016000 & 0.990543000 & 0.529102000 \\
\hline 1 & 5.429483000 & 0.048054000 & -0.987206000 & 1 & 3.477550000 & 2.120178000 & 0.876471000 & 1 & 2.730478000 & 2.312548000 & 1.489524000 \\
\hline 6 & 6.276165000 & -1.582499000 & 0.148195000 & 6 & 2.755812000 & 3.956118000 & -0.000225000 & 6 & 3.698934000 & 2.943152000 & -0.330337000 \\
\hline 1 & 6.137810000 & -2.090916000 & 1.104540000 & 1 & 2.212914000 & 4.300592000 & -0.881742000 & 1 & 3.872254000 & 2.588129000 & -1.347434000 \\
\hline 1 & 6.186374000 & -2.333061000 & -0.639759000 & 1 & 2.212705000 & 4.300978000 & 0.881011000 & 1 & 3.212471000 & 3.918096000 & -0.394585000 \\
\hline 1 & 7.296075000 & -1.197846000 & 0.123119000 & 1 & 3.731434000 & 4.441139000 & -0.000217000 & 1 & 4.671586000 & 3.088399000 & 0.138745000 \\
\hline 7 & 2.768130000 & 0.057398000 & -0.172054000 & 7 & 1.644851000 & 0.261781000 & 0.000376000 & 7 & 0.704727000 & 0.753464000 & 0.613282000 \\
\hline 1 & 2.886800000 & 0.538242000 & -1.056986000 & 1 & 2.121460000 & -0.107859000 & 0.818085000 & 1 & 0.921915000 & 0.790733000 & 1.600088000 \\
\hline 1 & 2.846643000 & 0.766468000 & 0.548735000 & 1 & 2.121820000 & -0.107841000 & -0.817134000 & 1 & 1.490819000 & -0.866802000 & 0.185652000 \\
\hline 1 & -3.498941000 & 1.601231000 & 0.103687000 & 1 & -0.207475000 & -0.558459000 & -2.155003000 & 1 & -0.677208000 & 1.393711000 & -1.616787000 \\
\hline 6 & -3.313578000 & -2.985200000 & -0.003003000 & 6 & -3.903845000 & 1.104346000 & -0.000055000 & 6 & -4.795781000 & -0.082638000 & -0.259513000 \\
\hline 7 & -3.809239000 & -4.025865000 & -0.005982000 & 7 & -4.958838000 & 1.574245000 & -0.000087000 & 7 & -5.922023000 & -0.260218000 & -0.432467000 \\
\hline
\end{tabular}


Table S12. Coordinates for the optimized reactants, products and transition states for the $n$ $\mathrm{RPhX}+\mathrm{NH}_{2}{ }^{\mathrm{n}} \mathrm{Pr}$ amination for $\mathrm{X}=\mathrm{I}$, where $\mathrm{n}=2$ (ortho), 3 (meta) or 4 (para) and $\mathrm{R}=-\mathrm{H},-\mathrm{Me}$, $-\mathrm{OMe},-\mathrm{F},-\mathrm{CF}_{3},-\mathrm{COOH}$, and $-\mathrm{CN}$.

\begin{tabular}{|c|c|c|c|c|c|c|c|c|c|c|c|}
\hline \multicolumn{4}{|c|}{ Reactant } & \multicolumn{4}{|c|}{ Transition State } & & & roduct & \\
\hline & & & & & $\boldsymbol{n}=$ & $2, \mathbf{R}=-$ & & & & & \\
\hline 6 & 2.418306000 & 3.144751000 & 0.000666000 & 6 & 0.337008000 & 3.443473000 & -0.000051000 & 6 & 4.204834000 & -1.012056000 & 0.356961000 \\
\hline 6 & 1.086979000 & 2.746522000 & -0.010744000 & 6 & 0.222209000 & 2.743608000 & -1.196707000 & 6 & 3.044207000 & -1.414111000 & 1.007943000 \\
\hline 6 & 3.425062000 & 2.186691000 & 0.012660000 & 6 & 0.222468000 & 2.743556000 & 1.196598000 & 6 & 4.152474000 & -0.010870000 & -0.606209000 \\
\hline 1 & 0.297521000 & 3.486508000 & -0.020043000 & 1 & 0.292234000 & 3.266885000 & -2.142075000 & 1 & 3.081161000 & -2.202343000 & 1.746912000 \\
\hline 1 & 4.464478000 & 2.487172000 & 0.021595000 & 1 & 0.292695000 & 3.266790000 & 2.141975000 & 1 & 5.051444000 & 0.299397000 & -1.120414000 \\
\hline 6 & 0.747950000 & 1.395942000 & -0.010356000 & 6 & -0.000007000 & 1.371461000 & -1.212770000 & 6 & 1.824703000 & -0.818868000 & 0.707702000 \\
\hline 6 & 3.106518000 & 0.832532000 & 0.013392000 & 6 & 0.000259000 & 1.371406000 & 1.212646000 & 6 & 2.940005000 & 0.593735000 & -0.913337000 \\
\hline 1 & -0.292023000 & 1.094487000 & -0.019587000 & 1 & -0.122429000 & 0.852006000 & -2.152320000 & 1 & 0.907377000 & -1.144046000 & 1.179956000 \\
\hline 1 & 3.889505000 & 0.088632000 & 0.022812000 & 1 & -0.121960000 & 0.851910000 & 2.152201000 & 1 & 2.893272000 & 1.373263000 & -1.663947000 \\
\hline 6 & 1.769666000 & 0.455519000 & 0.001767000 & 6 & -0.129751000 & 0.692612000 & -0.000063000 & 6 & 1.791281000 & 0.183973000 & -0.250809000 \\
\hline 53 & 1.284702000 & -1.617302000 & 0.003017000 & 53 & -2.016794000 & -0.948468000 & 0.000093000 & 53 & -2.038047000 & -1.178907000 & -0.084036000 \\
\hline 1 & 2.670969000 & 4.196497000 & 0.000273000 & 1 & 0.502371000 & 4.511398000 & -0.000045000 & 1 & 5.148851000 & -1.482948000 & 0.594313000 \\
\hline 6 & -3.918999000 & 0.761494000 & 0.005680000 & 6 & 2.496712000 & -0.482530000 & -0.000282000 & 6 & 0.139229000 & 1.972765000 & 0.339019000 \\
\hline 1 & -3.964096000 & 1.367823000 & 0.914397000 & 1 & 2.663067000 & 0.145330000 & -0.877002000 & 1 & -0.125534000 & 1.524039000 & 1.295155000 \\
\hline 1 & -4.001013000 & 1.461553000 & -0.830181000 & 1 & 2.663015000 & 0.145862000 & 0.876070000 & 1 & 1.024698000 & 2.593774000 & 0.472459000 \\
\hline 6 & -5.108831000 & -0.202425000 & -0.020770000 & 6 & 3.458524000 & -1.671188000 & 0.000112000 & 6 & -1.029190000 & 2.758509000 & -0.236927000 \\
\hline 1 & -5.018059000 & -0.901467000 & 0.816481000 & 1 & 3.260862000 & -2.295312000 & -0.875616000 & 1 & -1.857432000 & 2.071003000 & -0.416363000 \\
\hline 1 & -5.055973000 & -0.806876000 & -0.931676000 & 1 & 3.260766000 & -2.294790000 & 0.876191000 & 1 & -0.739370000 & 3.182811000 & -1.204083000 \\
\hline 6 & -6.457580000 & 0.512669000 & 0.047309000 & 6 & 4.920888000 & -1.227085000 & 0.000063000 & 6 & -1.468408000 & 3.876712000 & 0.706429000 \\
\hline 1 & -6.547478000 & 1.100260000 & 0.963345000 & 1 & 5.151531000 & -0.626317000 & -0.881368000 & 1 & -1.810831000 & 3.470837000 & 1.659013000 \\
\hline 1 & -6.585992000 & 1.195312000 & -0.795288000 & 1 & 5.151415000 & -0.625753000 & 0.881140000 & 1 & -0.655866000 & 4.577173000 & 0.909631000 \\
\hline 1 & -7.286158000 & -0.195975000 & 0.027157000 & 1 & 5.590625000 & -2.086827000 & 0.000381000 & 1 & -2.293062000 & 4.440576000 & 0.272418000 \\
\hline 7 & -2.590453000 & 0.147908000 & -0.055321000 & 7 & 1.078920000 & -0.855196000 & -0.000197000 & 7 & 0.513654000 & 0.823856000 & -0.560632000 \\
\hline 1 & -2.488227000 & -0.403786000 & -0.899753000 & 1 & 0.791847000 & -1.383934000 & 0.817325000 & 1 & 0.516351000 & 1.143538000 & -1.527538000 \\
\hline 1 & -2.453918000 & -0.490656000 & 0.720194000 & 1 & 0.791740000 & -1.383950000 & -0.817670000 & 1 & -0.314927000 & 0.089283000 & -0.483838000 \\
\hline & & & & & $\boldsymbol{n}=2$ & $\mathbf{P}=-2+$ & & & & & \\
\hline 6 & 2.789630000 & 2.801073000 & -0.452397000 & 6 & -0.305917000 & 3.402243000 & -0.440477000 & 6 & 4.328271000 & -0.856418000 & -0.117239000 \\
\hline 6 & 2.103185000 & 2.448577000 & 0.700753000 & 6 & -0.188908000 & 2.821716000 & 0.816461000 & 6 & 3.411375000 & -1.039082000 & 0.908260000 \\
\hline 6 & 2.924843000 & 1.882090000 & -1.483929000 & 6 & -0.219349000 & 2.591521000 & -1.565360000 & 6 & 3.964303000 & -0.148539000 & -1.254350000 \\
\hline 1 & 2.001328000 & 3.168888000 & 1.502488000 & 1 & -0.250618000 & 3.443544000 & 1.701697000 & 1 & 3.699595000 & -1.603452000 & 1.785349000 \\
\hline 1 & 3.458250000 & 2.141025000 & -2.388546000 & 1 & -0.290732000 & 3.017158000 & -2.558028000 & 1 & 4.664129000 & -0.005217000 & -2.065383000 \\
\hline 6 & 1.529961000 & 1.183336000 & 0.870198000 & 6 & 0.010557000 & 1.451155000 & 0.998249000 & 6 & 2.112048000 & -0.529130000 & 0.847892000 \\
\hline 6 & 2.369900000 & 0.615592000 & -1.351047000 & 6 & -0.016838000 & 1.223176000 & -1.435127000 & 6 & 2.681852000 & 0.370908000 & -1.344154000 \\
\hline 1 & 2.470907000 & -0.107359000 & -2.147117000 & 1 & 0.092246000 & 0.603394000 & -2.313321000 & 1 & 2.382228000 & 0.919635000 & -2.229036000 \\
\hline 6 & 1.685197000 & 0.283274000 & -0.188337000 & 6 & 0.120206000 & 0.677824000 & -0.161891000 & 6 & 1.775394000 & 0.183111000 & -0.307506000 \\
\hline 53 & 0.876116000 & -1.688315000 & -0.074917000 & 53 & 2.008005000 & -1.020339000 & -0.116755000 & 53 & -2.231438000 & -1.128779000 & -0.190063000 \\
\hline 1 & 3.217772000 & 3.790328000 & -0.542956000 & 1 & -0.456637000 & 4.467898000 & -0.537977000 & 1 & 5.322193000 & -1.273528000 & -0.030835000 \\
\hline 6 & -3.695147000 & 0.904169000 & 0.363026000 & 6 & -2.528024000 & -0.519877000 & -0.010195000 & 6 & 0.125586000 & 1.986500000 & 0.337485000 \\
\hline 1 & -4.183728000 & 1.569009000 & 1.080666000 & 1 & -2.701926000 & 0.050247000 & 0.903481000 & 1 & -0.119836000 & 1.638520000 & 1.337831000 \\
\hline 1 & -3.156733000 & 1.552824000 & -0.332965000 & 1 & -2.690052000 & 0.165073000 & -0.844661000 & 1 & 1.032002000 & 2.589268000 & 0.386397000 \\
\hline 6 & -4.761559000 & 0.113611000 & -0.402220000 & 6 & -3.494229000 & -1.702264000 & -0.094806000 & 6 & -1.037785000 & 2.761662000 & -0.265030000 \\
\hline 1 & -5.294413000 & -0.535419000 & 0.299920000 & 1 & -3.306551000 & -2.384049000 & 0.739087000 & 1 & -1.893592000 & 2.091905000 & -0.363400000 \\
\hline 1 & -4.265229000 & -0.549843000 & -1.116972000 & 1 & -3.289785000 & -2.267052000 & -1.008573000 & 1 & -0.766750000 & 3.093214000 & -1.272952000 \\
\hline 6 & -5.758443000 & 1.010860000 & -1.134678000 & 6 & -4.955298000 & -1.254334000 & -0.079482000 & 6 & -1.406131000 & 3.970752000 & 0.592625000 \\
\hline 1 & -6.289471000 & 1.662677000 & -0.437682000 & 1 & -5.194414000 & -0.715238000 & 0.838861000 & 1 & -1.727855000 & 3.661464000 & 1.587961000 \\
\hline 1 & -5.253122000 & 1.649762000 & -1.861993000 & 1 & -5.174580000 & -0.592697000 & -0.919286000 & 1 & -0.564315000 & 4.656496000 & 0.707514000 \\
\hline 1 & -6.504454000 & 0.424375000 & -1.672070000 & 1 & -5.627363000 & -2.109679000 & -0.146275000 & 1 & -2.226934000 & 4.524529000 & 0.138791000 \\
\hline 7 & -2.702699000 & 0.111110000 & 1.090227000 & 7 & -1.111846000 & -0.893016000 & -0.031803000 & 7 & 0.438386000 & 0.758843000 & -0.480189000 \\
\hline 1 & -2.191078000 & -0.493860000 & 0.457207000 & 1 & -0.839624000 & -1.419521000 & -0.854778000 & 1 & 0.325477000 & 0.997514000 & -1.464003000 \\
\hline 1 & -3.155210000 & -0.493601000 & 1.766223000 & 1 & -0.804256000 & -1.419781000 & 0.778182000 & 1 & -0.378837000 & 0.028166000 & -0.317652000 \\
\hline 6 & 0.787333000 & 0.857984000 & 2.135348000 & 6 & 0.139602000 & 0.868051000 & 2.375763000 & 6 & 1.159982000 & -0.757424000 & 1.991750000 \\
\hline 1 & -0.274919000 & 0.691145000 & 1.940168000 & 1 & -0.649661000 & 0.142345000 & 2.594846000 & 1 & 1.118096000 & 0.113748000 & 2.650696000 \\
\hline 1 & 1.179095000 & -0.046395000 & 2.603544000 & 1 & 1.091148000 & 0.344119000 & 2.485333000 & 1 & 0.146378000 & -0.973346000 & 1.651099000 \\
\hline 1 & 0.876327000 & 1.675992000 & 2.848805000 & 1 & 0.080636000 & 1.648060000 & 3.133247000 & 1 & 1.496155000 & -1.599566000 & 2.593868000 \\
\hline & & & & & $n=2$ & D - & & & & & \\
\hline 6 & -0.155700000 & 3.154131000 & -0.256810000 & 6 & -1.273466000 & 2.551526000 & -1.451868000 & 6 & 2.844517000 & -2.273181000 & -1.307173000 \\
\hline 6 & -0.285403000 & 2.268718000 & 0.807031000 & 6 & -1.087262000 & 2.353355000 & -0.081195000 & 6 & 3.180655000 & -1.598173000 & -0.136950000 \\
\hline 6 & 0.659701000 & 2.847790000 & -1.335375000 & 6 & -0.845071000 & 1.584327000 & -2.344096000 & 6 & 1.645492000 & -2.020304000 & -1.956922000 \\
\hline 1 & -0.931519000 & 2.519854000 & 1.633961000 & 1 & -1.432965000 & 3.100370000 & 0.618631000 & 1 & 4.113787000 & -1.816058000 & 0.359107000 \\
\hline 1 & 0.763293000 & 3.535392000 & -2.163033000 & 1 & -0.968190000 & 1.725434000 & -3.409712000 & 1 & 1.381903000 & -2.563320000 & -2.852786000 \\
\hline 6 & 0.408127000 & 1.056301000 & 0.807462000 & 6 & -0.474337000 & 1.202235000 & 0.392184000 & 6 & 2.307191000 & -0.649269000 & 0.384421000 \\
\hline 6 & 1.349233000 & 1.638883000 & -1.349315000 & 6 & -0.216687000 & 0.424502000 & -1.885970000 & 6 & 0.767718000 & -1.074897000 & -1.438228000 \\
\hline 1 & 1.985421000 & 1.385842000 & -2.184961000 & 1 & 0.166142000 & -0.298098000 & -2.592484000 & 1 & -0.192987000 & -0.892142000 & -1.900192000 \\
\hline 6 & 1.224222000 & 0.752782000 & -0.291696000 & 6 & 0.000003000 & 0.251678000 & -0.527073000 & 6 & 1.107302000 & -0.387492000 & -0.286331000 \\
\hline 53 & 2.273138000 & -1.087237000 & -0.343140000 & 53 & 2.306299000 & -0.539458000 & 0.005826000 & 53 & -2.920575000 & -0.414710000 & 0.268390000 \\
\hline 6 & -3.325323000 & -0.979982000 & -0.405866000 & 6 & -2.005143000 & -1.879478000 & -0.339099000 & 6 & 0.040528000 & 1.888332000 & -0.441763000 \\
\hline 1 & -2.873356000 & -0.087081000 & -0.844331000 & 1 & -2.119317000 & -1.508787000 & -1.361929000 & 1 & -0.212506000 & 1.641988000 & -1.470968000 \\
\hline 1 & -3.198381000 & -1.788418000 & -1.140568000 & 1 & -2.108758000 & -2.968037000 & -0.374613000 & 1 & -0.834510000 & 2.365791000 & -0.001145000 \\
\hline 6 & -4.814871000 & -0.736321000 & -0.189741000 & 6 & -3.081588000 & -1.280324000 & 0.557963000 & 6 & 1.276712000 & 2.768095000 & -0.357718000 \\
\hline 1 & -4.933817000 & 0.080998000 & 0.525188000 & 1 & -2.960659000 & -0.196748000 & 0.586021000 & 1 & 2.136846000 & 2.236862000 & -0.770022000 \\
\hline 1 & -5.256345000 & -1.621808000 & 0.279735000 & 1 & -2.936964000 & -1.641454000 & 1.580700000 & 1 & 1.507535000 & 2.974014000 & 0.690656000 \\
\hline 6 & -5.560388000 & -0.421007000 & -1.484880000 & 6 & -4.488196000 & -1.634918000 & 0.080518000 & 6 & 1.072159000 & 4.083515000 & -1.109755000 \\
\hline 1 & -5.163093000 & 0.478788000 & -1.959058000 & 1 & -4.670083000 & -1.254044000 & -0.925789000 & 1 & 0.870041000 & 3.906830000 & -2.167319000 \\
\hline 1 & -5.472372000 & -1.238147000 & -2.203864000 & 1 & -4.642219000 & -2.715608000 & 0.060902000 & 1 & 0.233291000 & 4.648610000 & -0.700860000 \\
\hline 1 & -6.622568000 & -0.256113000 & -1.300995000 & 1 & -5.243442000 & -1.205030000 & 0.738191000 & 1 & 1.960678000 & 4.710485000 & -1.041848000 \\
\hline 7 & -2.644073000 & -1.242567000 & 0.866788000 & 7 & -0.649898000 & -1.521806000 & 0.097365000 & 7 & 0.183095000 & 0.578783000 & 0.282981000 \\
\hline 1 & -1.649406000 & -1.369069000 & 0.719148000 & 1 & 0.078587000 & -2.128662000 & -0.260326000 & 1 & -0.845759000 & 0.148830000 & 0.324983000 \\
\hline 1 & -2.986464000 & -2.105676000 & 1.274268000 & 1 & -0.541541000 & -1.447626000 & 1.103269000 & 1 & 0.458899000 & 0.756190000 & 1.248287000 \\
\hline 1 & -0.701691000 & 4.087704000 & -0.235010000 & 1 & -1.749952000 & 3.455617000 & -1.802951000 & 1 & 3.529696000 & -3.010134000 & -1.703228000 \\
\hline 8 & 0.342684000 & 0.144780000 & 1.806365000 & 8 & -0.301584000 & 0.891203000 & 1.713164000 & 8 & 2.514672000 & 0.068881000 & 1.524754000 \\
\hline
\end{tabular}




\begin{tabular}{|c|c|c|c|c|c|c|c|c|c|c|}
\hline 6 & -0.454815000 & 0.434191000 & 2.952057000 & 6 & -0.569792000 & 1.891326000 & 2.682209000 & 6 & 3.663971000 & -0.219428000 \\
\hline 1 & -0.133924000 & 1.362245000 & 3.430144000 & 1 & 0.024155000 & 2.789179000 & 2.497025000 & 1 & 3.665700000 & -1.261898000 \\
\hline 1 & -1.511455000 & 0.481105000 & 2.685355000 & 1 & -1.630065000 & 2.158381000 & 2.705277000 & 1 & 4.582752000 & -0.001928000 \\
\hline 1 & -0.292293000 & -0.395234000 & 3.634816000 & 1 & -0.287363000 & 1.464022000 & 3.640705000 & 1 & 3.598571000 & 0.429783000 \\
\hline & & & & & $n=$ & R = & & & & \\
\hline 6 & -0.279428000 & 3.076734000 & -0.076650000 & 6 & -0.269434000 & 3.394733000 & -0.280384000 & 6 & -3.871390000 & -1.530638000 \\
\hline 6 & 0.424526000 & 1.889322000 & 0.077640000 & 6 & -0.201933000 & 2.731913000 & 0.943879000 & 6 & -3.977328000 & -0.441418000 \\
\hline 6 & -1.662998000 & 3.060456000 & -0.213756000 & 6 & -0.152487000 & 2.655251000 & -1.450287000 & 6 & -2.641688000 & -1.886389000 \\
\hline 1 & 1.501973000 & 1.859965000 & 0.188655000 & 1 & -0.290867000 & 3.257646000 & 1.885125000 & 1 & -4.918559000 & -0.145914000 \\
\hline 1 & -2.211124000 & 3.984779000 & -0.333598000 & 1 & -0.190755000 & 3.149613000 & -2.412098000 & 1 & -2.565137000 & -2.741435000 \\
\hline 6 & -0.278467000 & 0.696328000 & 0.091650000 & 6 & -0.021652000 & 1.366708000 & 0.969926000 & 6 & -2.834851000 & 0.275079000 \\
\hline 6 & -2.353184000 & 1.854224000 & -0.198049000 & 6 & 0.042035000 & 1.276894000 & -1.418178000 & 6 & -1.499238000 & -1.156643000 \\
\hline 1 & -3.428103000 & 1.838724000 & -0.304049000 & 1 & 0.181505000 & 0.729639000 & -2.339627000 & 1 & -0.527274000 & -1.437509000 \\
\hline 6 & -1.658662000 & 0.658956000 & -0.044505000 & 6 & 0.141072000 & 0.614221000 & -0.193169000 & 6 & -1.601347000 & -0.064640000 \\
\hline 53 & -2.681064000 & -1.188698000 & -0.018635000 & 53 & 2.000091000 & -1.003706000 & -0.089992000 & 53 & 2.301387000 & -1.057526000 \\
\hline 6 & 4.791819000 & 0.283772000 & -0.138099000 & 6 & -2.503027000 & -0.500458000 & -0.136433000 & 6 & -0.115884000 & 1.855626000 \\
\hline 1 & 4.459866000 & 0.193317000 & -1.175727000 & 1 & -2.685950000 & 0.214874000 & 0.666464000 & 1 & -1.047520000 & 2.373091000 \\
\hline 1 & 5.454297000 & 1.153049000 & -0.105991000 & 1 & -2.638203000 & 0.034897000 & -1.077473000 & 1 & 0.258005000 & 1.380368000 \\
\hline 6 & 5.578600000 & -0.969868000 & 0.254633000 & 6 & -3.475912000 & -1.675626000 & -0.039169000 & 6 & 0.923949000 & 2.789137000 \\
\hline 1 & 4.907830000 & -1.833836000 & 0.221599000 & 1 & -3.306050000 & -2.206890000 & 0.901041000 & 1 & 0.524947000 & 3.232399000 \\
\hline 1 & 5.903414000 & -0.873430000 & 1.295482000 & 1 & -3.263344000 & -2.387790000 & -0.841237000 & 1 & 1.803321000 & 2.203649000 \\
\hline 6 & 6.789442000 & -1.220497000 & -0.643300000 & 6 & -4.933198000 & -1.222747000 & -0.122008000 & 6 & 1.311768000 & 3.894600000 \\
\hline 1 & 6.488295000 & -1.350592000 & -1.684844000 & 1 & -5.178975000 & -0.532531000 & 0.686753000 & 1 & 0.448276000 & 4.493768000 \\
\hline 1 & 7.489620000 & -0.383259000 & -0.605579000 & 1 & -5.136871000 & -0.714736000 & -1.066155000 & 1 & 1.758857000 & 3.476999000 \\
\hline 1 & 7.331115000 & -2.117663000 & -0.341892000 & 1 & -5.610511000 & -2.073403000 & -0.049898000 & 1 & 2.042633000 & 4.564955000 \\
\hline 7 & 3.615448000 & 0.586371000 & 0.681235000 & 7 & -1.090744000 & -0.887281000 & -0.057492000 & 7 & -0.432312000 & 0.728275000 \\
\hline 1 & 3.881370000 & 0.700469000 & 1.652961000 & 1 & -0.788609000 & -1.509386000 & -0.799979000 & 1 & 0.471352000 & 0.078123000 \\
\hline 1 & 2.953818000 & -0.181715000 & 0.651711000 & 1 & -0.830192000 & -1.309461000 & 0.828047000 & 1 & -0.549391000 & 1.112410000 \\
\hline 9 & 0.409821000 & -0.453904000 & 0.243589000 & 9 & 0.002917000 & 0.716050000 & 2.162009000 & 9 & -2.895009000 & 1.339614000 \\
\hline 1 & 0.257608000 & 4.015145000 & -0.089385000 & 1 & -0.409830000 & 4.465124000 & -0.312053000 & 1 & -4.755706000 & -2.104772000 \\
\hline & & & & & $n=$ & 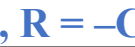 & & & & \\
\hline 6 & 0.291180000 & 2.840178000 & 0.021984000 & 6 & 0.394538000 & 2.374071000 & 2.356450000 & 6 & $\begin{array}{l}-2.669926000 \\
\end{array}$ & -2.659337000 \\
\hline 6 & 0.659809000 & 1.514716000 & 0.205615000 & 6 & 0.237625000 & 2.509458000 & 0.990859000 & 6 & -3.183770000 & -1.591788000 \\
\hline 6 & -1.036709000 & 3.163534000 & -0.210516000 & 6 & 0.314123000 & 1.095765000 & 2.910467000 & 6 & -1.324822000 & -2.684557000 \\
\hline 1 & 1.699000000 & 1.273246000 & 0.388222000 & 1 & 0.288547000 & 3.488593000 & 0.536546000 & 1 & -4.228053000 & -1.571421000 \\
\hline 1 & -1.335395000 & 4.193183000 & -0.355388000 & 1 & 0.418178000 & 0.957510000 & 3.979191000 & 1 & -0.915301000 & -3.519672000 \\
\hline 6 & -0.294687000 & 0.497749000 & 0.159227000 & 6 & 0.006798000 & 1.408482000 & 0.157622000 & 6 & -2.358903000 & -0.540038000 \\
\hline 6 & -1.998110000 & 2.161461000 & -0.258928000 & 6 & 0.081692000 & -0.011478000 & 2.120145000 & 6 & -0.487511000 & -1.642502000 \\
\hline 1 & -3.032435000 & 2.413377000 & -0.439323000 & 1 & -0.018617000 & -0.986535000 & 2.575136000 & 1 & 0.571627000 & -1.666704000 \\
\hline 6 & -1.633667000 & 0.832806000 & -0.075502000 & 6 & -0.094736000 & 0.121226000 & 0.728018000 & 6 & -1.004642000 & -0.574365000 \\
\hline 53 & -3.212786000 & -0.588734000 & -0.175622000 & 53 & -1.937612000 & -1.283613000 & -0.012462000 & 53 & 2.940024000 & -0.671777000 \\
\hline 6 & 4.827059000 & 0.318686000 & -0.260204000 & 6 & 2.546730000 & -0.709742000 & 0.059223000 & 6 & 0.182214000 & 1.519810000 \\
\hline 1 & 4.136698000 & -0.340943000 & -0.791577000 & 1 & 2.670404000 & 0.271941000 & -0.398551000 & 1 & -0.769567000 & 1.780816000 \\
\hline 1 & 5.078164000 & 1.123269000 & -0.956596000 & 1 & 2.716664000 & -0.589991000 & 1.130342000 & 1 & 0.810806000 & 1.016260000 \\
\hline 6 & 6.096794000 & -0.457592000 & 0.100064000 & 6 & 3.538534000 & -1.708205000 & -0.536878000 & 6 & 0.887929000 & 2.736125000 \\
\hline 1 & 5.836539000 & -1.258740000 & 0.798648000 & 1 & 3.337491000 & -1.821857000 & -1.605260000 & 1 & 0.241519000 & 3.206286000 \\
\hline 1 & 6.781197000 & 0.207491000 & 0.636262000 & 1 & 3.379006000 & -2.690668000 & -0.083755000 & 1 & 1.794978000 & 2.405234000 \\
\hline 6 & 6.800390000 & -1.046826000 & -1.121819000 & 6 & 4.987812000 & -1.271217000 & -0.326703000 & 6 & 1.236599000 & 3.747426000 \\
\hline 1 & 6.146951000 & -1.738400000 & -1.657409000 & 1 & 5.180930000 & -0.304979000 & -0.795532000 & 1 & 0.346236000 & 4.091769000 \\
\hline 1 & 7.096163000 & -0.263540000 & -1.822956000 & 1 & 5.223757000 & -1.179270000 & 0.734827000 & 1 & 1.919514000 & 3.313784000 \\
\hline 1 & 7.700116000 & -1.594085000 & -0.838894000 & 1 & 5.679080000 & -1.994137000 & -0.759200000 & 1 & 1.725395000 & 4.620139000 \\
\hline 7 & 4.095348000 & 0.912888000 & 0.863116000 & 7 & 1.141120000 & -1.094413000 & -0.115892000 & 7 & -0.085598000 & 0.497818000 \\
\hline 1 & 4.690890000 & 1.555666000 & 1.373053000 & 1 & 0.908471000 & -1.984682000 & 0.312692000 & 1 & 0.910018000 & 0.059712000 \\
\hline 1 & 3.819046000 & 0.192061000 & 1.520587000 & 1 & 0.852224000 & -1.124358000 & -1.087684000 & 1 & -0.401937000 & 0.970235000 \\
\hline 1 & 1.046761000 & 3.612308000 & 0.061774000 & 1 & 0.568649000 & 3.239833000 & 2.977850000 & 1 & -3.321664000 & -3.470994000 \\
\hline 6 & 0.162068000 & -0.927961000 & 0.366110000 & 6 & -0.209524000 & 1.671862000 & -1.301819000 & 6 & -2.935753000 & 0.613489000 \\
\hline 9 & 1.494281000 & -1.005366000 & 0.571129000 & 9 & 0.379496000 & 2.819457000 & -1.700504000 & 9 & -4.220027000 & 0.437775000 \\
\hline 9 & -0.422022000 & -1.500574000 & 1.438309000 & 9 & 0.314909000 & 0.689925000 & -2.097167000 & 9 & -2.848201000 & 1.778102000 \\
\hline 9 & -0.105395000 & -1.709915000 & -0.699041000 & 9 & -1.507821000 & 1.771692000 & -1.645568000 & 9 & -2.260010000 & 0.821728000 \\
\hline & & & & & $n=2$ & & 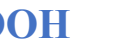 & & & \\
\hline 6 & -0.388763000 & 2.706746000 & -0.407341000 & 6 & -0.297540000 & 2.962010000 & -1.568162000 & 6 & -3.060197000 & -2.355262000 \\
\hline 6 & -0.679575000 & 1.370654000 & -0.627317000 & 6 & -0.168379000 & 2.763660000 & -0.213042000 & 6 & -3.480448000 & -1.293242000 \\
\hline 6 & 0.886021000 & 3.075605000 & 0.000321000 & 6 & -0.212713000 & 1.849083000 & -2.413919000 & 6 & -1.728395000 & -2.440638000 \\
\hline 1 & -1.676984000 & 1.075190000 & -0.929153000 & 1 & -0.222461000 & 3.590718000 & 0.481293000 & 1 & -4.508182000 & -1.211798000 \\
\hline 1 & 1.126873000 & 4.115074000 & 0.179310000 & 1 & -0.297037000 & 1.974404000 & -3.486266000 & 1 & -1.382931000 & -3.274894000 \\
\hline 6 & 0.300372000 & 0.381941000 & -0.467525000 & 6 & 0.034894000 & 1.486790000 & 0.344795000 & 6 & -2.590848000 & -0.292049000 \\
\hline 6 & 1.861801000 & 2.107308000 & 0.197628000 & 6 & 0.008838000 & 0.584611000 & -1.914326000 & 6 & -0.825985000 & -1.459383000 \\
\hline 1 & 2.843874000 & 2.394792000 & 0.542189000 & 1 & 0.127206000 & -0.249100000 & -2.591402000 & 1 & 0.221303000 & -1.532886000 \\
\hline 6 & 1.577817000 & 0.765893000 & -0.038236000 & 6 & 0.157395000 & 0.374998000 & -0.525286000 & 6 & -1.251773000 & -0.389606000 \\
\hline 6 & -4.973454000 & 0.369546000 & 0.044156000 & 6 & -2.514454000 & -0.606797000 & -0.200627000 & 6 & 0.075173000 & 1.588234000 \\
\hline 1 & -4.438826000 & 0.676780000 & 0.947211000 & 1 & -2.677910000 & 0.295317000 & 0.390610000 & 1 & -0.869306000 & 1.943255000 \\
\hline 1 & -5.597931000 & 1.220533000 & -0.243221000 & 1 & -2.625388000 & -0.325618000 & -1.249527000 & 1 & 0.607357000 & 1.025820000 \\
\hline 6 & -5.870366000 & -0.828308000 & 0.372434000 & 6 & -3.531261000 & -1.684169000 & 0.177374000 & 6 & 0.929115000 & 2.730157000 \\
\hline 1 & -5.238899000 & -1.673344000 & 0.662044000 & 1 & -3.393952000 & -1.956437000 & 1.227157000 & 1 & 0.370745000 & 3.271981000 \\
\hline 1 & -6.397318000 & -1.135951000 & -0.536508000 & 1 & -3.333188000 & -2.587605000 & -0.406263000 & 1 & 1.817827000 & 2.310298000 \\
\hline 6 & -6.881216000 & -0.532802000 & 1.479805000 & 6 & -4.970073000 & -1.224736000 & -0.055013000 & 6 & 1.333765000 & 3.688927000 \\
\hline 1 & -6.378978000 & -0.253121000 & 2.408328000 & 1 & -5.203656000 & -0.339515000 & 0.538874000 & 1 & 0.462957000 & 4.117276000 \\
\hline 1 & -7.541984000 & 0.291410000 & 1.202366000 & 1 & -5.141599000 & -0.976036000 & -1.103721000 & 1 & 1.939155000 & 3.180191000 \\
\hline 1 & -7.506987000 & -1.400513000 & 1.691960000 & 1 & -5.678343000 & -2.005750000 & 0.220840000 & 1 & 1.925982000 & 4.511733000 \\
\hline 7 & -3.980665000 & 0.158918000 & -1.010158000 & 7 & -1.120063000 & -1.007418000 & -0.000814000 & 7 & -0.240743000 & 0.603199000 \\
\hline 1 & -4.434200000 & -0.110105000 & -1.875706000 & 1 & -0.845932000 & -1.820415000 & -0.541744000 & 1 & 0.712619000 & 0.094819000 \\
\hline 1 & -3.346097000 & -0.592914000 & -0.761625000 & 1 & -0.875779000 & -1.153888000 & 0.973993000 & 1 & -0.535091000 & 1.120727000 \\
\hline 1 & -1.158111000 & 3.452695000 & -0.550141000 & 1 & -0.455578000 & 3.951948000 & -1.969973000 & 1 & -3.766376000 & -3.117633000 \\
\hline 6 & -0.142741000 & -1.009680000 & -0.775257000 & 6 & 0.114744000 & 1.432252000 & 1.810193000 & 6 & -3.182796000 & 0.773497000 \\
\hline 8 & -1.244882000 & -1.440157000 & -0.527516000 & 8 & 0.244014000 & 2.379422000 & 2.549858000 & 8 & -4.348757000 & 0.860693000 \\
\hline 8 & 0.783059000 & -1.746109000 & -1.416971000 & 8 & -0.023057000 & 0.168544000 & 2.328123000 & 8 & -2.277848000 & 1.678402000 \\
\hline 1 & 0.396594000 & -2.624004000 & -1.554139000 & 1 & 0.090440000 & 0.263257000 & 3.284524000 & 1 & -2.771250000 & 2.297239000 \\
\hline 53 & 3.146582000 & -0.594344000 & 0.429662000 & 53 & 1.978816000 & -1.124892000 & -0.140348000 & 53 & 2.701275000 & -0.803303000 \\
\hline
\end{tabular}




\begin{tabular}{|c|c|c|c|c|c|c|c|c|}
\hline 6 & 1.092002000 & 3.398892000 & -0.162232000 & 6 & -0.347009000 & 3.341670000 & -0.462105000 & 6 \\
\hline 6 & 0.467129000 & 2.616592000 & 0.793164000 & 6 & -0.282682000 & 2.727813000 & 0.771686000 & 6 \\
\hline 6 & 1.921580000 & 2.803542000 & -1.105278000 & 6 & -0.176709000 & 2.557572000 & -1.607481000 & 6 \\
\hline 1 & -0.182984000 & 3.062290000 & 1.532367000 & 1 & -0.417357000 & 3.297513000 & 1.681237000 & 1 \\
\hline 1 & 2.413274000 & 3.406719000 & -1.856454000 & 1 & -0.214204000 & 3.018418000 & -2.586447000 & 1 \\
\hline 6 & 0.662638000 & 1.229171000 & 0.817954000 & 6 & -0.041605000 & 1.349493000 & 0.890013000 & 6 \\
\hline 6 & 2.128036000 & 1.429411000 & -1.093425000 & 6 & 0.066591000 & 1.200395000 & -1.529062000 & 6 \\
\hline 1 & 2.774071000 & 0.972917000 & -1.828299000 & 1 & 0.243086000 & 0.631133000 & -2.430332000 & 1 \\
\hline 6 & 1.502772000 & 0.640287000 & -0.137221000 & 6 & 0.166775000 & 0.565185000 & -0.274742000 & 6 \\
\hline 53 & 1.847752000 & -1.443361000 & -0.158402000 & 53 & 2.020906000 & -0.946609000 & -0.195860000 & 53 \\
\hline 1 & 0.931604000 & 4.467728000 & -0.171921000 & 1 & -0.523412000 & 4.404042000 & -0.539777000 & 1 \\
\hline 6 & -3.651395000 & 0.092459000 & -0.562341000 & 6 & -2.464561000 & -0.513270000 & -0.283744000 & 6 \\
\hline 1 & -3.654222000 & 1.020094000 & 0.017007000 & 1 & -2.645151000 & 0.205144000 & 0.516465000 & 1 \\
\hline 1 & -3.689902000 & 0.392567000 & -1.613747000 & 1 & -2.589202000 & 0.016986000 & -1.228748000 & 1 \\
\hline 6 & -4.906992000 & -0.717251000 & -0.222186000 & 6 & -3.442326000 & -1.683443000 & -0.183855000 & 6 \\
\hline 1 & -4.862988000 & -1.012811000 & 0.830331000 & 1 & -3.278229000 & -2.209271000 & 0.760181000 & 1 \\
\hline 1 & -4.897744000 & -1.645513000 & -0.802303000 & 1 & -3.232496000 & -2.400881000 & -0.982026000 & 1 \\
\hline 6 & -6.202309000 & 0.047461000 & -0.491768000 & 6 & -4.896478000 & -1.221550000 & -0.272138000 & 6 \\
\hline 1 & -6.249431000 & 0.964173000 & 0.099896000 & 1 & -5.139709000 & -0.528164000 & 0.534446000 & 1 \\
\hline 1 & -6.282857000 & 0.330485000 & -1.543591000 & 1 & -5.095109000 & -0.715533000 & -1.218459000 & 1 \\
\hline 1 & -7.079405000 & -0.551052000 & -0.242871000 & 1 & -5.578298000 & -2.068335000 & -0.198874000 & 1 \\
\hline 7 & -2.369241000 & -0.572280000 & -0.330948000 & 7 & -1.052969000 & -0.911718000 & -0.192822000 & 7 \\
\hline 1 & -2.313827000 & -1.433068000 & -0.863033000 & 1 & -0.755910000 & -1.528995000 & -0.942335000 & 1 \\
\hline 1 & -2.268105000 & -0.826197000 & 0.645431000 & 1 & -0.824588000 & -1.363747000 & 0.688958000 & 1 \\
\hline 6 & -0.009658000 & 0.469376000 & 1.822067000 & 6 & -0.053284000 & 0.736339000 & 2.170715000 & 6 \\
\hline 7 & -0.572353000 & -0.100555000 & 2.650963000 & 7 & -0.122702000 & 0.204678000 & 3.194329000 & 7 \\
\hline & & & & & $n=$ & $\mathbf{R}=$ & & \\
\hline 6 & 2.418306000 & 3.144751000 & 0.000666000 & 6 & 0.337008000 & 3.443473000 & -0.000051000 & 6 \\
\hline 6 & 1.086979000 & 2.746522000 & -0.010744000 & 6 & 0.222209000 & 2.743608000 & -1.196707000 & 6 \\
\hline 6 & 3.425062000 & 2.186691000 & 0.012660000 & 6 & 0.222468000 & 2.743556000 & 1.196598000 & 6 \\
\hline 1 & 0.297521000 & 3.486508000 & -0.020043000 & 1 & 0.292234000 & 3.266885000 & -2.142075000 & 1 \\
\hline 1 & 4.464478000 & 2.487172000 & 0.021595000 & 1 & 0.292695000 & 3.266790000 & 2.141975000 & 1 \\
\hline 6 & 0.747950000 & 1.395942000 & -0.010356000 & 6 & -0.000007000 & 1.371461000 & -1.212770000 & 6 \\
\hline 6 & 3.106518000 & 0.832532000 & 0.013392000 & 6 & 0.000259000 & 1.371406000 & 1.212646000 & 6 \\
\hline 1 & -0.292023000 & 1.094487000 & -0.019587000 & 1 & -0.122429000 & 0.852006000 & -2.152320000 & 1 \\
\hline 1 & 3.889505000 & 0.088632000 & 0.022812000 & 1 & -0.121960000 & 0.851910000 & 2.152201000 & 1 \\
\hline 6 & 1.769666000 & 0.455519000 & 0.001767000 & 6 & -0.129751000 & 0.692612000 & -0.000063000 & 6 \\
\hline 53 & 1.284702000 & -1.617302000 & 0.003017000 & 53 & -2.016794000 & -0.948468000 & 0.000093000 & 53 \\
\hline 1 & 2.670969000 & 4.196497000 & 0.000273000 & 1 & 0.502371000 & 4.511398000 & -0.000045000 & 1 \\
\hline 6 & -3.918999000 & 0.761494000 & 0.005680000 & 6 & 2.496712000 & -0.482530000 & -0.000282000 & 6 \\
\hline 1 & -3.964096000 & 1.367823000 & 0.914397000 & 1 & 2.663067000 & 0.145330000 & -0.877002000 & 1 \\
\hline 1 & -4.001013000 & 1.461553000 & -0.830181000 & 1 & 2.663015000 & 0.145862000 & 0.876070000 & 1 \\
\hline 6 & -5.108831000 & -0.202425000 & -0.020770000 & 6 & 3.458524000 & -1.671188000 & 0.000112000 & 6 \\
\hline 1 & -5.018059000 & -0.901467000 & 0.816481000 & 1 & 3.260862000 & -2.295312000 & -0.875616000 & 1 \\
\hline 1 & -5.055973000 & -0.806876000 & -0.931676000 & 1 & 3.260766000 & -2.294790000 & 0.876191000 & 1 \\
\hline 6 & -6.457580000 & 0.512669000 & 0.047309000 & 6 & 4.920888000 & -1.227085000 & 0.000063000 & 6 \\
\hline 1 & -6.547478000 & 1.100260000 & 0.963345000 & 1 & 5.151531000 & -0.626317000 & -0.881368000 & 1 \\
\hline 1 & -6.585992000 & 1.195312000 & -0.795288000 & 1 & 5.151415000 & -0.625753000 & 0.881140000 & 1 \\
\hline 1 & -7.286158000 & -0.195975000 & 0.027157000 & 1 & 5.590625000 & -2.086827000 & 0.000381000 & 1 \\
\hline 7 & -2.590453000 & 0.147908000 & -0.055321000 & 7 & 1.078920000 & -0.855196000 & -0.000197000 & 7 \\
\hline 1 & -2.488227000 & -0.403786000 & -0.899753000 & 1 & 0.791847000 & -1.383934000 & 0.817325000 & 1 \\
\hline 1 & -2.453918000 & -0.490656000 & 0.720194000 & 1 & 0.791740000 & -1.383950000 & -0.817670000 & 1 \\
\hline & & & & & $\boldsymbol{n}=?$ & $N--1$ & & \\
\hline 6 & 2.965927000 & 2.509500000 & -0.155209000 & 6 & 0.504783000 & 3.086594000 & 0.853091000 & 6 \\
\hline 6 & 1.586108000 & 2.423682000 & 0.039203000 & 6 & 0.360309000 & 2.694031000 & -0.479189000 & 6 \\
\hline 6 & 3.736082000 & 1.364533000 & -0.299323000 & 6 & 0.335756000 & 2.153820000 & 1.869038000 & 6 \\
\hline 1 & 4.804716000 & 1.445571000 & -0.449476000 & 1 & 0.432053000 & 2.458557000 & 2.903749000 & 1 \\
\hline 6 & 0.991348000 & 1.161577000 & 0.087427000 & 6 & 0.059549000 & 1.364259000 & -0.772574000 & 6 \\
\hline 6 & 3.147549000 & 0.104134000 & -0.251361000 & 6 & 0.030220000 & 0.826574000 & 1.593605000 & 6 \\
\hline 1 & 3.746941000 & -0.787204000 & -0.362266000 & 1 & -0.132057000 & 0.123021000 & 2.397043000 & 1 \\
\hline 6 & 1.776843000 & 0.025434000 & -0.056991000 & 6 & -0.123806000 & 0.445870000 & 0.261027000 & 6 \\
\hline 53 & 0.853933000 & -1.891988000 & 0.025563000 & 53 & -2.099217000 & -1.048204000 & -0.106983000 & 53 \\
\hline 6 & -3.730215000 & 0.893529000 & -0.091733000 & 6 & 2.433830000 & -0.854336000 & 0.021062000 & 6 \\
\hline 1 & -3.994529000 & 1.942823000 & 0.065697000 & 1 & 2.656385000 & -0.054685000 & -0.687198000 & 1 \\
\hline 1 & -3.462832000 & 0.803145000 & -1.147807000 & 1 & 2.609958000 & -0.450760000 & 1.019483000 & 1 \\
\hline 6 & -4.949950000 & 0.014262000 & 0.200097000 & 6 & 3.331264000 & -2.064819000 & -0.235740000 & 6 \\
\hline 1 & -5.207167000 & 0.104323000 & 1.260206000 & 1 & 3.125758000 & -2.462041000 & -1.233601000 & 1 \\
\hline 1 & -4.678815000 & -1.033487000 & 0.038217000 & 1 & 3.077786000 & -2.858945000 & 0.471888000 & 1 \\
\hline 6 & -6.162451000 & 0.373218000 & -0.657941000 & 6 & 4.814695000 & -1.717669000 & -0.113487000 & 6 \\
\hline 1 & -6.471546000 & 1.407399000 & -0.492292000 & 1 & 5.100740000 & -0.946447000 & -0.830783000 & 1 \\
\hline 1 & -5.940139000 & 0.262632000 & -1.721301000 & 1 & 5.053290000 & -1.347280000 & 0.884980000 & 1 \\
\hline 1 & -7.015642000 & -0.266826000 & -0.430843000 & 1 & 5.437439000 & -2.592275000 & -0.300609000 & 1 \\
\hline 7 & -2.528746000 & 0.611287000 & 0.697439000 & 7 & 0.998864000 & -1.133388000 & -0.082397000 & 7 \\
\hline 1 & -2.221950000 & -0.344580000 & 0.552619000 & 1 & 0.661244000 & -1.816231000 & 0.588365000 & 1 \\
\hline 1 & -2.723328000 & 0.704267000 & 1.688059000 & 1 & 0.704149000 & -1.442427000 & -1.003092000 & 1 \\
\hline 1 & -0.077661000 & 1.080975000 & 0.237832000 & 1 & -0.081457000 & 1.063401000 & -1.801774000 & 1 \\
\hline 1 & 3.440596000 & 3.482204000 & -0.193677000 & 1 & 0.737979000 & 4.115412000 & 1.092052000 & 1 \\
\hline 6 & 0.746778000 & 3.666033000 & 0.191104000 & 6 & 0.481041000 & 3.699267000 & -1.596020000 & 6 \\
\hline 1 & 0.781071000 & 4.276207000 & -0.714111000 & 1 & 1.224062000 & 4.461924000 & -1.361612000 & 1 \\
\hline 1 & -0.293992000 & 3.415478000 & 0.388478000 & 1 & 0.763637000 & 3.224808000 & -2.535689000 & 1 \\
\hline 1 & 1.108952000 & 4.288014000 & 1.012103000 & 1 & -0.470377000 & 4.210982000 & -1.761188000 & 1 \\
\hline & & & & & $n=3$, & $\mathrm{R}=-\mathrm{-}$ & Ie & \\
\hline 6 & -2.665075000 & 2.157973000 & 0.105813000 & 6 & 2.423767000 & 1.351237000 & 1.148719000 & 6 \\
\hline 6 & -3.302046000 & 0.930822000 & -0.068916000 & 6 & 2.114193000 & 1.239874000 & -0.207095000 & 6 \\
\hline 6 & -1.280476000 & 2.192550000 & 0.247007000 & 6 & 1.495877000 & 0.881548000 & 2.076583000 & 6 \\
\hline 1 & -0.787915000 & 3.146556000 & 0.382779000 & 1 & 1.714133000 & 0.974991000 & 3.133105000 & 1 \\
\hline 6 & -2.548955000 & -0.247628000 & -0.101458000 & 6 & 0.908148000 & 0.669133000 & -0.621917000 & 6 \\
\hline 6 & -0.512851000 & 1.036318000 & 0.218360000 & 6 & 0.292263000 & 0.310271000 & 1.698904000 & 6 \\
\hline 1 & 0.561894000 & 1.084740000 & 0.330937000 & 1 & -0.422168000 & -0.009314000 & 2.442655000 & 1 \\
\hline 6 & -1.176098000 & -0.174071000 & 0.041998000 & 6 & -0.000666000 & 0.227573000 & 0.331942000 & 6 \\
\hline
\end{tabular}




\begin{tabular}{|c|c|c|c|c|c|c|c|c|}
\hline 53 & -0.052803000 & -1.981481000 & -0.012394000 & 53 & -2.351985000 & 0.875838000 & -0.183487000 & 53 \\
\hline 6 & 4.153037000 & 1.474894000 & -0.051761000 & 6 & 0.584812000 & -2.566403000 & 0.055148000 & 6 \\
\hline 1 & 3.897560000 & 1.494882000 & -1.114511000 & 1 & 1.406939000 & -2.197693000 & -0.560566000 & 1 \\
\hline 1 & 4.212636000 & 2.519675000 & 0.265417000 & 1 & 0.918194000 & -2.517620000 & 1.092671000 & 1 \\
\hline 6 & 5.521937000 & 0.811714000 & 0.129264000 & 6 & 0.225846000 & -4.001504000 & -0.330061000 & 6 \\
\hline 1 & 5.455183000 & -0.232738000 & -0.190391000 & 1 & -0.124853000 & -4.019334000 & -1.365685000 & 1 \\
\hline 1 & 5.767997000 & 0.791042000 & 1.195619000 & 1 & -0.610384000 & -4.343386000 & 0.285922000 & 1 \\
\hline 6 & 6.634128000 & 1.519288000 & -0.643801000 & 6 & 1.409998000 & -4.954151000 & -0.169010000 & 6 \\
\hline 1 & 6.427270000 & 1.525478000 & -1.716003000 & 1 & 2.249036000 & -4.651734000 & -0.797931000 & 1 \\
\hline 1 & 6.738882000 & 2.557939000 & -0.323149000 & 1 & 1.760830000 & -4.977803000 & 0.864120000 & 1 \\
\hline 1 & 7.597147000 & 1.029389000 & -0.496180000 & 1 & 1.134422000 & -5.970685000 & -0.449215000 & 1 \\
\hline 7 & 3.036093000 & 0.853783000 & 0.662850000 & 7 & -0.517656000 & -1.611404000 & -0.089592000 & 7 \\
\hline 1 & 3.215615000 & 0.835435000 & 1.660387000 & 1 & -1.305804000 & -1.791982000 & 0.523983000 & 1 \\
\hline 1 & 2.914437000 & -0.110516000 & 0.373735000 & 1 & -0.879001000 & -1.544480000 & -1.035813000 & 1 \\
\hline 1 & -3.062334000 & -1.187138000 & -0.238944000 & 1 & 0.688506000 & 0.642255000 & -1.678918000 & 1 \\
\hline 1 & -3.227494000 & 3.078400000 & 0.133173000 & 1 & 3.346035000 & 1.796473000 & 1.484567000 & 1 \\
\hline 8 & -4.645211000 & 0.768923000 & -0.217205000 & 8 & 2.923898000 & 1.665539000 & -1.217659000 & 8 \\
\hline 6 & -5.472384000 & 1.921230000 & -0.197128000 & 6 & 4.147059000 & 2.299986000 & -0.882433000 & 6 \\
\hline 1 & -5.398980000 & 2.450077000 & 0.756657000 & 1 & 3.980285000 & 3.203203000 & -0.290345000 & 1 \\
\hline 1 & -5.222576000 & 2.607234000 & -1.010607000 & 1 & 4.811348000 & 1.627413000 & -0.333270000 & 1 \\
\hline 1 & -6.489017000 & 1.561646000 & -0.330582000 & 1 & 4.612320000 & 2.570426000 & -1.826717000 & 1 \\
\hline & & & & & $n=$ & 3, $\mathbf{R}=$ & & \\
\hline 6 & -1.034576000 & 3.275669000 & -0.001249000 & 6 & 0.538265000 & 3.119181000 & 0.741366000 & 6 \\
\hline 6 & -0.027178000 & 2.328472000 & -0.044647000 & 6 & 0.386327000 & 2.640191000 & -0.548350000 & 6 \\
\hline 6 & -2.350278000 & 2.831746000 & 0.048632000 & 6 & 0.349538000 & 2.209711000 & 1.777964000 & 6 \\
\hline 9 & 1.255305000 & 2.748548000 & -0.093744000 & 9 & 0.548180000 & 3.492775000 & -1.584005000 & 9 \\
\hline 1 & -3.157184000 & 3.551212000 & 0.083130000 & 1 & 0.440338000 & 2.544936000 & 2.803177000 & 1 \\
\hline 6 & -0.271840000 & 0.964781000 & -0.040375000 & 6 & 0.066197000 & 1.327514000 & -0.844491000 & 6 \\
\hline 6 & -2.644899000 & 1.472349000 & 0.054770000 & 6 & 0.029201000 & 0.880256000 & 1.541351000 & 6 \\
\hline 1 & -3.669931000 & 1.135912000 & 0.093658000 & 1 & -0.150050000 & 0.209506000 & 2.368687000 & 1 \\
\hline 6 & -1.598567000 & 0.558426000 & 0.009959000 & 6 & -0.129311000 & 0.441514000 & 0.220012000 & 6 \\
\hline 53 & -2.036933000 & -1.520108000 & 0.018427000 & 53 & -2.104583000 & -1.011040000 & -0.087918000 & 53 \\
\hline 6 & 3.760992000 & -0.281747000 & 0.066981000 & 6 & 2.415323000 & -0.838889000 & 0.019256000 & 6 \\
\hline 1 & 3.757821000 & 0.601446000 & -0.576794000 & 1 & 2.631471000 & -0.083654000 & -0.737629000 & 1 \\
\hline 1 & 3.681762000 & 0.091920000 & 1.091050000 & 1 & 2.596699000 & -0.377097000 & 0.990966000 & 1 \\
\hline 6 & 5.082961000 & -1.035444000 & -0.101154000 & 6 & 3.307591000 & -2.064614000 & -0.172027000 & 6 \\
\hline 1 & 5.151519000 & -1.407475000 & -1.128328000 & 1 & 3.092894000 & -2.520874000 & -1.142301000 & 1 \\
\hline 1 & 5.073057000 & -1.919347000 & 0.544523000 & 1 & 3.059760000 & -2.814040000 & 0.584688000 & 1 \\
\hline 6 & 6.305181000 & -0.176014000 & 0.218927000 & 6 & 4.792459000 & -1.713100000 & -0.084508000 & 6 \\
\hline 1 & 6.355530000 & 0.697643000 & -0.434094000 & 1 & 5.072345000 & -0.986356000 & -0.848942000 & 1 \\
\hline 1 & 6.275239000 & 0.184836000 & 1.249074000 & 1 & 5.041341000 & -1.285195000 & 0.888024000 & 1 \\
\hline 1 & 7.231446000 & -0.737369000 & 0.092144000 & 1 & 5.411555000 & -2.598698000 & -0.225863000 & 1 \\
\hline 7 & 2.544934000 & -1.047763000 & -0.221815000 & 7 & 0.978069000 & -1.125506000 & -0.057234000 & 7 \\
\hline 1 & 2.477071000 & -1.854259000 & 0.388598000 & 1 & 0.652914000 & -1.778366000 & 0.649180000 & 1 \\
\hline 1 & 2.566037000 & -1.405243000 & -1.170340000 & 1 & 0.680098000 & -1.484301000 & -0.959241000 & 1 \\
\hline 1 & 0.557824000 & 0.267539000 & -0.076947000 & 1 & -0.079204000 & 1.035985000 & -1.873752000 & 1 \\
\hline 1 & -0.785807000 & 4.326908000 & -0.006993000 & 1 & 0.779070000 & 4.156277000 & 0.915911000 & 1 \\
\hline & & & & & $\boldsymbol{n}=$ & 11 & & \\
\hline 6 & 1.540486000 & -2.876285000 & 0.355074000 & 6 & -2.127051000 & -0.442666000 & 1.714817000 & 6 \\
\hline 6 & 0.446607000 & -2.074683000 & 0.054077000 & 6 & -2.001190000 & -0.484619000 & 0.330322000 & 6 \\
\hline 6 & 2.786845000 & -2.289939000 & 0.537423000 & 6 & -0.978085000 & -0.247078000 & 2.474233000 & 6 \\
\hline 1 & 3.644193000 & -2.906024000 & 0.771645000 & 1 & -1.044577000 & -0.230383000 & 3.554315000 & 1 \\
\hline 6 & 0.580298000 & -0.690731000 & -0.067962000 & 6 & -0.767609000 & -0.336101000 & -0.294156000 & 6 \\
\hline 6 & 2.942162000 & -0.915169000 & 0.419694000 & 6 & 0.265784000 & -0.088862000 & 1.882216000 & 6 \\
\hline 1 & -0.284045000 & -0.078590000 & -0.304351000 & 1 & -0.695617000 & -0.414202000 & -1.368700000 & 1 \\
\hline 1 & 3.912766000 & -0.463045000 & 0.561009000 & 1 & 1.146901000 & 0.026361000 & 2.497131000 & 1 \\
\hline 6 & 1.834483000 & -0.130075000 & 0.117111000 & 6 & 0.377295000 & -0.147829000 & 0.486490000 & 6 \\
\hline 53 & 2.080972000 & 1.970195000 & -0.068606000 & 53 & 2.299111000 & -1.494716000 & -0.265864000 & 53 \\
\hline 1 & 1.420925000 & -3.945296000 & 0.444627000 & 1 & -3.088864000 & -0.573902000 & 2.184855000 & 1 \\
\hline 6 & -3.293043000 & 1.295163000 & 0.195364000 & 6 & 0.555741000 & 2.664585000 & 0.062512000 & 6 \\
\hline 1 & -3.326276000 & 0.498356000 & 0.942475000 & 1 & -0.413431000 & 2.539808000 & -0.422628000 & 1 \\
\hline 1 & -2.827301000 & 2.154254000 & 0.685334000 & 1 & 0.367663000 & 2.747160000 & 1.133895000 & 1 \\
\hline 6 & -4.719498000 & 1.659791000 & -0.225166000 & 6 & 1.265253000 & 3.912949000 & -0.460371000 & 6 \\
\hline 1 & -5.177137000 & 0.794719000 & -0.714946000 & 1 & 1.459466000 & 3.796121000 & -1.530036000 & 1 \\
\hline 1 & -4.676302000 & 2.452226000 & -0.979138000 & 1 & 2.241352000 & 4.004062000 & 0.024060000 & 1 \\
\hline 6 & -5.589557000 & 2.109760000 & 0.947808000 & 6 & 0.447682000 & 5.181395000 & -0.219355000 & 6 \\
\hline 1 & -5.673405000 & 1.323881000 & 1.701222000 & 1 & -0.520883000 & 5.128580000 & -0.719107000 & 1 \\
\hline 1 & -5.168167000 & 2.990603000 & 1.436650000 & 1 & 0.264880000 & 5.338223000 & 0.844987000 & 1 \\
\hline 1 & -6.598648000 & 2.363784000 & 0.621622000 & 1 & 0.970209000 & 6.058906000 & -0.599284000 & 1 \\
\hline 7 & -2.399078000 & 0.857118000 & -0.880216000 & 7 & 1.305956000 & 1.419781000 & -0.148399000 & 7 \\
\hline 1 & -2.314098000 & 1.577085000 & -1.588710000 & 1 & 2.199421000 & 1.393680000 & 0.333842000 & 1 \\
\hline 1 & -2.773231000 & 0.035626000 & -1.341799000 & 1 & 1.486385000 & 1.214471000 & -1.126545000 & 1 \\
\hline 6 & -0.918401000 & -2.679667000 & -0.140338000 & 6 & -3.225345000 & -0.632544000 & -0.532945000 & 6 \\
\hline 9 & -1.798941000 & -2.245812000 & 0.789060000 & 9 & -3.687905000 & 0.576522000 & -0.942426000 & 9 \\
\hline 9 & -1.445459000 & -2.359699000 & -1.344785000 & 9 & -2.985542000 & -1.345264000 & -1.651949000 & 9 \\
\hline 9 & -0.902216000 & -4.024124000 & -0.066108000 & 9 & -4.242370000 & -1.233793000 & 0.112177000 & 9 \\
\hline & & & & & $n=3$ & --0 & ОH & \\
\hline 6 & -2.264803000 & 2.285530000 & 0.168616000 & 6 & -2.373285000 & -0.731736000 & 1.580069000 & 6 \\
\hline 6 & -2.983735000 & 1.104943000 & -0.018032000 & 6 & -2.197833000 & -0.740083000 & 0.195714000 & 6 \\
\hline 6 & -0.886825000 & 2.239236000 & 0.307315000 & 6 & -1.287198000 & -0.427967000 & 2.388379000 & 6 \\
\hline 1 & -0.325358000 & 3.152370000 & 0.451944000 & 1 & -1.396896000 & -0.429945000 & 3.465143000 & 1 \\
\hline 6 & -2.316428000 & -0.121230000 & -0.064925000 & 6 & -0.956586000 & -0.450861000 & -0.373273000 & 6 \\
\hline 6 & -0.206595000 & 1.024379000 & 0.262512000 & 6 & -0.041571000 & -0.134002000 & 1.846699000 & 6 \\
\hline 1 & 0.871113000 & 1.000237000 & 0.372150000 & 1 & 0.798326000 & 0.064304000 & 2.497226000 & 1 \\
\hline 6 & -0.937311000 & -0.142938000 & 0.076270000 & 6 & 0.126206000 & -0.163352000 & 0.458645000 & 6 \\
\hline 53 & 0.072798000 & -2.012643000 & 0.003256000 & 53 & 2.216841000 & -1.310249000 & -0.233362000 & 53 \\
\hline 6 & 4.358260000 & 1.381104000 & -0.070853000 & 6 & 0.066603000 & 2.668760000 & 0.044092000 & 6 \\
\hline 1 & 4.082286000 & 1.394178000 & -1.128491000 & 1 & -0.855544000 & 2.457723000 & -0.499624000 & 1 \\
\hline 1 & 4.380925000 & 2.425962000 & 0.250131000 & 1 & -0.193255000 & 2.720356000 & 1.102560000 & 1 \\
\hline 6 & 5.754960000 & 0.772122000 & 0.083112000 & 6 & 0.677765000 & 3.988320000 & -0.425715000 & 6 \\
\hline
\end{tabular}




\begin{tabular}{|c|c|c|c|c|c|c|c|c|}
\hline 1 & 5.723460000 & -0.273442000 & -0.238474000 & 1 & 0.948762000 & 3.905167000 & -1.481712000 & 1 \\
\hline 1 & 6.021362000 & 0.758779000 & 1.144654000 & 1 & 1.607685000 & 4.171294000 & 0.119662000 & 1 \\
\hline 6 & 6.823674000 & 1.524745000 & -0.708385000 & 6 & -0.277359000 & 5.165167000 & -0.228474000 & 6 \\
\hline 1 & 6.596880000 & 1.525192000 & -1.776515000 & 1 & -1.201584000 & 5.022982000 & -0.790887000 & 1 \\
\hline 1 & 6.893738000 & 2.565838000 & -0.386410000 & 1 & -0.543244000 & 5.287108000 & 0.822899000 & 1 \\
\hline 1 & 7.807507000 & 1.072436000 & -0.579929000 & 1 & 0.176933000 & 6.096338000 & -0.566165000 & 1 \\
\hline 7 & 3.280460000 & 0.711490000 & 0.662631000 & 7 & 0.942454000 & 1.504237000 & -0.128516000 & 7 \\
\hline 1 & 3.480467000 & 0.700908000 & 1.656592000 & 1 & 1.806709000 & 1.557193000 & 0.401940000 & 1 \\
\hline 1 & 3.201474000 & -0.258060000 & 0.375798000 & 1 & 1.192837000 & 1.318528000 & -1.094819000 & 1 \\
\hline 1 & -2.876023000 & -1.031478000 & -0.209400000 & 1 & -0.837017000 & -0.495966000 & -1.444224000 & 1 \\
\hline 1 & -2.802237000 & 3.222034000 & 0.201651000 & 1 & -3.341460000 & -0.967545000 & 1.993553000 & 1 \\
\hline 6 & -4.459852000 & 1.195753000 & -0.162929000 & 6 & -3.370095000 & -1.074390000 & -0.655328000 & 6 \\
\hline 8 & -5.093680000 & 2.222204000 & -0.132221000 & 8 & -4.471209000 & -1.344743000 & -0.241511000 & 8 \\
\hline 8 & -5.051877000 & -0.010715000 & -0.337870000 & 8 & -3.095535000 & -1.043710000 & -1.984624000 & 8 \\
\hline 1 & -6.000747000 & 0.160901000 & -0.420162000 & 1 & -3.917956000 & -1.285550000 & -2.433506000 & 1 \\
\hline & & & & & $n=3$ & $\mathbf{R}=-$ & & \\
\hline 6 & -2.123783000 & 2.635498000 & -0.106900000 & 6 & 1.031157000 & 2.659476000 & 1.287387000 & 6 \\
\hline 6 & -3.062421000 & 1.608221000 & 0.026741000 & 6 & 0.880889000 & 2.421066000 & -0.081939000 & 6 \\
\hline 6 & -0.777539000 & 2.320839000 & -0.203632000 & 6 & 0.603919000 & 1.680163000 & 2.174728000 & 6 \\
\hline 1 & -0.046320000 & 3.110846000 & -0.307212000 & 1 & 0.693306000 & 1.846124000 & 3.240329000 & 1 \\
\hline 6 & -2.645802000 & 0.272473000 & 0.063434000 & 6 & 0.316575000 & 1.233507000 & -0.556041000 & 6 \\
\hline 6 & -0.346690000 & 0.997459000 & -0.169321000 & 6 & 0.044167000 & 0.490587000 & 1.732025000 & 6 \\
\hline 1 & 0.710185000 & 0.771062000 & -0.247212000 & 1 & -0.312984000 & -0.235941000 & 2.447927000 & 1 \\
\hline 6 & -1.292881000 & -0.012231000 & -0.034774000 & 6 & -0.116391000 & 0.266238000 & 0.356375000 & 6 \\
\hline 53 & -0.662866000 & -2.038462000 & 0.024608000 & 53 & -2.309589000 & -0.681282000 & -0.209813000 & 53 \\
\hline 6 & 4.207630000 & 1.111365000 & 0.028843000 & 6 & 2.138704000 & -1.448854000 & 0.023944000 & 6 \\
\hline 1 & 4.190128000 & 2.040399000 & -0.547104000 & 1 & 2.528746000 & -0.657183000 & -0.617184000 & 1 \\
\hline 1 & 4.010564000 & 1.392893000 & 1.066556000 & 1 & 2.373143000 & -1.172068000 & 1.052866000 & 1 \\
\hline 6 & 5.597318000 & 0.476734000 & -0.071703000 & 6 & 2.772637000 & -2.792638000 & -0.332741000 & 6 \\
\hline 1 & 5.784499000 & 0.194476000 & -1.112498000 & 1 & 2.503100000 & -3.057910000 & -1.358672000 & 1 \\
\hline 1 & 5.605184000 & -0.453534000 & 0.504678000 & 1 & 2.353635000 & -3.571906000 & 0.309755000 & 1 \\
\hline 6 & 6.711071000 & 1.399548000 & 0.421275000 & 6 & 4.294000000 & -2.766104000 & -0.189082000 & 6 \\
\hline 1 & 6.743799000 & 2.324190000 & -0.158641000 & 1 & 4.740225000 & -2.015041000 & -0.842809000 & 1 \\
\hline 1 & 6.563646000 & 1.672360000 & 1.468233000 & 1 & 4.591467000 & -2.535271000 & 0.835086000 & 1 \\
\hline 1 & 7.688416000 & 0.923313000 & 0.338711000 & 1 & 4.725416000 & -3.731963000 & -0.450333000 & 1 \\
\hline 7 & 3.088067000 & 0.281446000 & -0.428040000 & 7 & 0.675241000 & -1.427419000 & -0.105956000 & 7 \\
\hline 1 & 3.049504000 & -0.583312000 & 0.100351000 & 1 & 0.203541000 & -2.092252000 & 0.500170000 & 1 \\
\hline 1 & 3.217922000 & 0.015875000 & -1.398085000 & 1 & 0.348644000 & -1.601414000 & -1.052009000 & 1 \\
\hline 1 & -3.377704000 & -0.513947000 & 0.167198000 & 1 & 0.180317000 & 1.096933000 & -1.618375000 & 1 \\
\hline 1 & -2.456094000 & 3.663134000 & -0.133533000 & 1 & 1.460185000 & 3.586061000 & 1.635863000 & 1 \\
\hline 6 & -4.454917000 & 1.915801000 & 0.127540000 & 6 & 1.308552000 & 3.405379000 & -1.028120000 & 6 \\
\hline 7 & -5.577171000 & 2.165199000 & 0.208802000 & 7 & 1.663931000 & 4.192688000 & -1.791435000 & 7 \\
\hline & & & & & $n=$ & $\mathbf{R}=$ & & \\
\hline 6 & 2.418306000 & 3.144751000 & 0.000666000 & 6 & 0.337008000 & 3.443473000 & -0.000051000 & 6 \\
\hline 6 & 1.086979000 & 2.746522000 & -0.010744000 & 6 & 0.222209000 & 2.743608000 & -1.196707000 & 6 \\
\hline 6 & 3.425062000 & 2.186691000 & 0.012660000 & 6 & 0.222468000 & 2.743556000 & 1.196598000 & 6 \\
\hline 1 & 0.297521000 & 3.486508000 & -0.020043000 & 1 & 0.292234000 & 3.266885000 & -2.142075000 & 1 \\
\hline 1 & 4.464478000 & 2.487172000 & 0.021595000 & 1 & 0.292695000 & 3.266790000 & 2.141975000 & 1 \\
\hline 6 & 0.747950000 & 1.395942000 & -0.010356000 & 6 & -0.000007000 & 1.371461000 & -1.212770000 & 6 \\
\hline 6 & 3.106518000 & 0.832532000 & 0.013392000 & 6 & 0.000259000 & 1.371406000 & 1.212646000 & 6 \\
\hline 1 & -0.292023000 & 1.094487000 & -0.019587000 & 1 & -0.122429000 & 0.852006000 & -2.152320000 & 1 \\
\hline 1 & 3.889505000 & 0.088632000 & 0.022812000 & 1 & -0.121960000 & 0.851910000 & 2.152201000 & 1 \\
\hline 6 & 1.769666000 & 0.455519000 & 0.001767000 & 6 & -0.129751000 & 0.692612000 & -0.000063000 & 6 \\
\hline 53 & 1.284702000 & -1.617302000 & 0.003017000 & 53 & -2.016794000 & -0.948468000 & 0.000093000 & 53 \\
\hline 1 & 2.670969000 & 4.196497000 & 0.000273000 & 1 & 0.502371000 & 4.511398000 & -0.000045000 & 1 \\
\hline 6 & -3.918999000 & 0.761494000 & 0.005680000 & 6 & 2.496712000 & -0.482530000 & -0.000282000 & 6 \\
\hline 1 & -3.964096000 & 1.367823000 & 0.914397000 & 1 & 2.663067000 & 0.145330000 & -0.877002000 & 1 \\
\hline 1 & -4.001013000 & 1.461553000 & -0.830181000 & 1 & 2.663015000 & 0.145862000 & 0.876070000 & 1 \\
\hline 6 & -5.108831000 & -0.202425000 & -0.020770000 & 6 & 3.458524000 & -1.671188000 & 0.000112000 & 6 \\
\hline 1 & -5.018059000 & -0.901467000 & 0.816481000 & 1 & 3.260862000 & -2.295312000 & -0.875616000 & 1 \\
\hline 1 & -5.055973000 & -0.806876000 & -0.931676000 & 1 & 3.260766000 & -2.294790000 & 0.876191000 & 1 \\
\hline 6 & -6.457580000 & 0.512669000 & 0.047309000 & 6 & 4.920888000 & -1.227085000 & 0.000063000 & 6 \\
\hline 1 & -6.547478000 & 1.100260000 & 0.963345000 & 1 & 5.151531000 & -0.626317000 & -0.881368000 & 1 \\
\hline 1 & -6.585992000 & 1.195312000 & -0.795288000 & 1 & 5.151415000 & -0.625753000 & 0.881140000 & 1 \\
\hline 1 & -7.286158000 & -0.195975000 & 0.027157000 & 1 & 5.590625000 & -2.086827000 & 0.000381000 & 1 \\
\hline 7 & -2.590453000 & 0.147908000 & -0.055321000 & 7 & 1.078920000 & -0.855196000 & -0.000197000 & 7 \\
\hline 1 & -2.488227000 & -0.403786000 & -0.899753000 & 1 & 0.791847000 & -1.383934000 & 0.817325000 & 1 \\
\hline 1 & -2.453918000 & -0.490656000 & 0.720194000 & 1 & 0.791740000 & -1.383950000 & -0.817670000 & 1 \\
\hline & & & & & $n=$ & $N--$ & & \\
\hline 6 & 2.340929000 & 2.702737000 & $\begin{array}{l}-0.009714000 \\
\end{array}$ & 6 & -1.148745000 & 2.967838000 & -0.000024000 & 6 \\
\hline 6 & 1.003337000 & 2.313301000 & 0.054511000 & 6 & -0.844551000 & 2.306153000 & 1.189734000 & 6 \\
\hline 6 & 3.310282000 & 1.702259000 & -0.077687000 & 6 & -0.844640000 & 2.305983000 & -1.189712000 & 6 \\
\hline 1 & 0.227878000 & 3.068206000 & 0.101725000 & 1 & -1.055602000 & 2.786618000 & 2.138158000 & 1 \\
\hline 1 & 4.357455000 & 1.973296000 & -0.134620000 & 1 & -1.055752000 & 2.786319000 & -2.138188000 & 1 \\
\hline 6 & 0.630085000 & 0.973105000 & 0.056547000 & 6 & -0.260027000 & 1.044892000 & 1.208647000 & 6 \\
\hline 6 & 2.962649000 & 0.356216000 & -0.077646000 & 6 & -0.260129000 & 1.044718000 & -1.208495000 & 6 \\
\hline 1 & 3.730572000 & -0.401432000 & -0.133675000 & 1 & -0.010427000 & 0.578574000 & -2.150870000 & 1 \\
\hline 6 & 1.621622000 & 0.004365000 & -0.009460000 & 6 & 0.051007000 & 0.424951000 & 0.000108000 & 6 \\
\hline 53 & 1.083306000 & -2.054054000 & -0.015115000 & 53 & 2.315264000 & -0.657807000 & -0.000047000 & 53 \\
\hline 6 & -4.054651000 & 0.849956000 & -0.034600000 & 6 & -2.160418000 & -1.428495000 & 0.000025000 & 6 \\
\hline 1 & -4.068180000 & 1.740300000 & 0.600032000 & 1 & -2.490857000 & -0.868173000 & 0.876165000 & 1 \\
\hline 1 & -3.948724000 & 1.211137000 & -1.060999000 & 1 & -2.490609000 & -0.868388000 & -0.876346000 & 1 \\
\hline 6 & -5.385234000 & 0.104934000 & 0.107320000 & 6 & -2.765559000 & -2.832438000 & 0.000112000 & 6 \\
\hline 1 & -5.482250000 & -0.255491000 & 1.136222000 & 1 & -2.407189000 & -3.380068000 & 0.876048000 & 1 \\
\hline 1 & -5.363047000 & -0.785697000 & -0.528404000 & 1 & -2.406887000 & -3.380305000 & -0.875552000 & 1 \\
\hline 6 & -6.593744000 & 0.967521000 & -0.253945000 & 6 & -4.293620000 & -2.800287000 & -0.000157000 & 6 \\
\hline 1 & -6.656544000 & 1.848419000 & 0.388381000 & 1 & -4.678137000 & -2.283699000 & 0.880975000 & 1 \\
\hline 1 & -6.534816000 & 1.317002000 & -1.286817000 & 1 & -4.677831000 & -2.283956000 & -0.881572000 & 1 \\
\hline 1 & -7.526367000 & 0.412896000 & -0.145732000 & 1 & -4.706147000 & -3.809025000 & -0.000081000 & 1 \\
\hline 7 & -2.849430000 & 0.085909000 & 0.295100000 & 7 & -0.695658000 & -1.402145000 & 0.000229000 & 7 \\
\hline
\end{tabular}




\begin{tabular}{|c|c|c|c|c|c|c|c|c|}
\hline 1 & -2.770899000 & -0.733363000 & -0.296811000 & 1 & -0.273447000 & -1.830318000 & -0.817273000 & 1 \\
\hline 1 & -2.890792000 & -0.252806000 & 1.249711000 & 1 & -0.273710000 & -1.830164000 & 0.817945000 & 1 \\
\hline 1 & -0.417453000 & 0.704914000 & 0.107061000 & 1 & -0.010262000 & 0.578880000 & 2.151070000 & 1 \\
\hline 6 & 2.728489000 & 4.157986000 & 0.018508000 & 6 & -1.742010000 & 4.352963000 & -0.000106000 & 6 \\
\hline 1 & 3.658470000 & 4.333268000 & -0.522352000 & 1 & -2.362393000 & 4.520713000 & -0.881294000 & 1 \\
\hline 1 & 1.954668000 & 4.783933000 & -0.425767000 & 1 & -2.362374000 & 4.520828000 & 0.881075000 & 1 \\
\hline 1 & 2.878254000 & 4.504618000 & 1.044427000 & 1 & -0.965629000 & 5.123305000 & -0.000163000 & 1 \\
\hline & & & & & $n=4$, & $\mathbf{R}=$ & Me & \\
\hline 6 & 3.073501000 & 1.461742000 & 0.016949000 & 6 & 2.838681000 & -0.516885000 & 0.279184000 & 6 \\
\hline 6 & 1.730523000 & 1.746402000 & 0.276590000 & 6 & 2.046373000 & -0.417552000 & 1.424393000 & 6 \\
\hline 6 & 3.458270000 & 0.149379000 & -0.251233000 & 6 & 2.227381000 & -0.449877000 & -0.968951000 & 6 \\
\hline 1 & 1.446657000 & 2.769274000 & 0.482143000 & 1 & 2.521331000 & -0.485231000 & 2.393882000 & 1 \\
\hline 1 & 4.487727000 & -0.101724000 & -0.456050000 & 1 & 2.802127000 & -0.538245000 & -1.878897000 & 1 \\
\hline 6 & 0.778699000 & 0.740482000 & 0.270680000 & 6 & 0.673141000 & -0.256340000 & 1.333131000 & 6 \\
\hline 6 & 2.502828000 & -0.865384000 & -0.257214000 & 6 & 0.844373000 & -0.285406000 & -1.074636000 & 6 \\
\hline 1 & 2.809264000 & -1.880229000 & -0.465522000 & 1 & 0.386233000 & -0.270170000 & -2.052944000 & 1 \\
\hline 6 & 1.176133000 & -0.566704000 & 0.002400000 & 6 & 0.072851000 & -0.214196000 & 0.075224000 & 6 \\
\hline 53 & -0.266226000 & -2.129007000 & -0.010860000 & 53 & -2.048817000 & -1.594909000 & -0.057263000 & 53 \\
\hline 6 & -3.683694000 & 1.798398000 & -0.016335000 & 6 & -0.402470000 & 2.648879000 & -0.002893000 & 6 \\
\hline 1 & -3.599111000 & 2.878321000 & 0.133101000 & 1 & 0.205382000 & 2.666274000 & 0.903363000 & 1 \\
\hline 1 & -3.371927000 & 1.609597000 & -1.046914000 & 1 & 0.289263000 & 2.634036000 & -0.846877000 & 1 \\
\hline 6 & -5.145116000 & 1.374635000 & 0.158261000 & 6 & -1.300811000 & 3.884609000 & -0.069210000 & 6 \\
\hline 1 & -5.448499000 & 1.563880000 & 1.192875000 & 1 & -1.991566000 & 3.874007000 & 0.778234000 & 1 \\
\hline 1 & -5.220870000 & 0.293096000 & 0.009541000 & 1 & -1.916820000 & 3.834495000 & -0.971215000 & 1 \\
\hline 6 & -6.094186000 & 2.095525000 & -0.798157000 & 6 & -0.495262000 & 5.183465000 & -0.064598000 & 6 \\
\hline 1 & -6.058132000 & 3.176933000 & -0.650508000 & 1 & 0.106790000 & 5.272607000 & 0.841304000 & 1 \\
\hline 1 & -5.831502000 & 1.896764000 & -1.839257000 & 1 & 0.181807000 & 5.231480000 & -0.919259000 & 1 \\
\hline 1 & -7.126667000 & 1.776575000 & -0.651491000 & 1 & -1.152664000 & 6.051379000 & -0.112934000 & 1 \\
\hline 7 & -2.714412000 & 1.147224000 & 0.867388000 & 7 & -1.123858000 & 1.374535000 & -0.015042000 & 7 \\
\hline 1 & -2.721529000 & 0.142434000 & 0.729990000 & 1 & -1.665172000 & 1.210950000 & -0.857724000 & 1 \\
\hline 1 & -2.951324000 & 1.309653000 & 1.839547000 & 1 & -1.747521000 & 1.241888000 & 0.774451000 & 1 \\
\hline 1 & -0.257552000 & 0.981247000 & 0.472178000 & 1 & 0.077854000 & -0.215907000 & 2.233886000 & 1 \\
\hline 8 & 3.923417000 & 2.524413000 & 0.048035000 & 8 & 4.182459000 & -0.681295000 & 0.487925000 & 8 \\
\hline 6 & 5.300426000 & 2.299924000 & -0.205857000 & 6 & 5.018374000 & -0.831659000 & -0.643123000 & 6 \\
\hline 1 & 5.738499000 & 1.621641000 & 0.531166000 & 1 & 4.742099000 & -1.709604000 & -1.234714000 & 1 \\
\hline 1 & 5.462733000 & 1.896400000 & -1.208854000 & 1 & 4.992937000 & 0.053901000 & -1.285740000 & 1 \\
\hline 1 & 5.781448000 & 3.271240000 & -0.128870000 & 1 & 6.026755000 & -0.963300000 & -0.259107000 & 1 \\
\hline & & & & & $n=$ & D - & & \\
\hline 6 & 2.777079000 & 2.379914000 & -0.025474000 & 6 & 3.272826000 & 1.091945000 & -0.120859000 & 6 \\
\hline 6 & 1.410245000 & 2.296258000 & 0.168601000 & 6 & 2.903365000 & 0.514578000 & 1.080802000 & 6 \\
\hline 6 & 3.562699000 & 1.257512000 & -0.217316000 & 6 & 2.372781000 & 1.217367000 & -1.162618000 & 6 \\
\hline 1 & 0.831113000 & 3.197050000 & 0.314529000 & 1 & 3.628862000 & 0.414117000 & 1.876127000 & 1 \\
\hline 1 & 4.627819000 & 1.363637000 & -0.365726000 & 1 & 2.690491000 & 1.655056000 & -2.098839000 & 1 \\
\hline 6 & 0.800836000 & 1.045368000 & 0.172015000 & 6 & 1.602339000 & 0.053710000 & 1.249915000 & 6 \\
\hline 6 & 2.955386000 & 0.006633000 & -0.213903000 & 6 & 1.067061000 & 0.761552000 & -1.004591000 & 6 \\
\hline 1 & -0.269146000 & 0.970830000 & 0.322513000 & 1 & 1.320702000 & -0.426127000 & 2.175914000 & 1 \\
\hline 1 & 3.553934000 & -0.879994000 & -0.362064000 & 1 & 0.376862000 & 0.825905000 & -1.831811000 & 1 \\
\hline 6 & 1.582721000 & -0.087176000 & -0.019428000 & 6 & 0.694125000 & 0.163549000 & 0.196471000 & 6 \\
\hline 53 & 0.669210000 & -2.006010000 & -0.016012000 & 53 & -0.654877000 & -1.954678000 & -0.136485000 & 53 \\
\hline 9 & 3.364555000 & 3.594312000 & -0.028035000 & 9 & 4.542827000 & 1.535390000 & -0.281981000 & 9 \\
\hline 6 & -3.798222000 & 1.158676000 & -0.021726000 & 6 & -1.865050000 & 1.583637000 & -0.058142000 & 6 \\
\hline 1 & -3.871916000 & 2.194940000 & 0.318938000 & 1 & -1.310015000 & 2.346081000 & -0.606931000 & 1 \\
\hline 1 & -3.556451000 & 1.205697000 & -1.086747000 & 1 & -2.127506000 & 0.790436000 & -0.759216000 & 1 \\
\hline 6 & -5.151348000 & 0.465541000 & 0.162613000 & 6 & -3.127544000 & 2.184679000 & 0.560469000 & 6 \\
\hline 1 & -5.383126000 & 0.417779000 & 1.231258000 & 1 & -2.846196000 & 2.952243000 & 1.287058000 & 1 \\
\hline 1 & -5.068757000 & -0.570556000 & -0.179931000 & 1 & -3.657938000 & 1.406871000 & 1.115911000 & 1 \\
\hline 6 & -6.287017000 & 1.166948000 & -0.581287000 & 6 & -4.052823000 & 2.786532000 & -0.496708000 & 6 \\
\hline 1 & -6.408411000 & 2.196135000 & -0.237121000 & 1 & -3.555037000 & 3.583844000 & -1.051532000 & 1 \\
\hline 1 & -6.094395000 & 1.199802000 & -1.655615000 & 1 & -4.374181000 & 2.030833000 & -1.215028000 & 1 \\
\hline 1 & -7.237840000 & 0.654436000 & -0.431887000 & 1 & -4.946083000 & 3.209715000 & -0.037757000 & 1 \\
\hline 7 & -2.660827000 & 0.542267000 & 0.666446000 & 7 & -0.946525000 & 0.992468000 & 0.921337000 & 7 \\
\hline 1 & -2.531234000 & -0.414959000 & 0.357664000 & 1 & -1.371441000 & 0.218705000 & 1.424606000 & 1 \\
\hline 1 & -2.829204000 & 0.501023000 & 1.665423000 & 1 & -0.582265000 & 1.679159000 & 1.575239000 & 1 \\
\hline & & & & & $n$ & & & \\
\hline 6 & -2.667785000 & 0.726911000 & -0.050912000 & 6 & 2.311553000 & -0.379051000 & 0.000009000 & 6 \\
\hline 6 & -1.364602000 & 1.131149000 & 0.218990000 & 6 & 1.602461000 & -0.330861000 & 1.199378000 & 6 \\
\hline 6 & -2.941442000 & -0.612380000 & -0.308573000 & 6 & 1.602480000 & -0.330858000 & -1.199370000 & 6 \\
\hline 1 & -1.149847000 & 2.172242000 & 0.413438000 & 1 & 2.131259000 & -0.390312000 & 2.140752000 & 1 \\
\hline 1 & -3.951891000 & -0.928877000 & -0.523830000 & 1 & 2.131296000 & -0.390309000 & -2.140734000 & 1 \\
\hline 6 & -0.326906000 & 0.206464000 & 0.231220000 & 6 & 0.222309000 & -0.233417000 & 1.212990000 & 6 \\
\hline 6 & -1.914041000 & -1.546407000 & -0.299336000 & 6 & 0.222330000 & -0.233418000 & -1.213004000 & 6 \\
\hline 1 & -2.127114000 & -2.585083000 & -0.503406000 & 1 & -0.307136000 & -0.242351000 & -2.154837000 & 1 \\
\hline 6 & -0.617254000 & -1.126818000 & -0.028459000 & 6 & -0.484859000 & -0.191910000 & -0.000015000 & 6 \\
\hline 53 & 0.948452000 & -2.559996000 & -0.017839000 & 53 & -2.470090000 & -1.608458000 & 0.000005000 & 53 \\
\hline 6 & 3.657323000 & 2.183339000 & -0.032770000 & 6 & -0.883833000 & 2.615593000 & 0.000017000 & 6 \\
\hline 1 & 3.487293000 & 1.832100000 & -1.053782000 & 1 & -0.235132000 & 2.605126000 & 0.876764000 & 1 \\
\hline 1 & 3.165842000 & 3.157243000 & 0.039196000 & 1 & -0.235006000 & 2.605104000 & -0.876637000 & 1 \\
\hline 6 & 5.160362000 & 2.353348000 & 0.204101000 & 6 & -1.774156000 & 3.857357000 & -0.000061000 & 6 \\
\hline 1 & 5.644267000 & 1.374316000 & 0.132280000 & 1 & -2.428096000 & 3.834298000 & 0.875980000 & 1 \\
\hline 1 & 5.321398000 & 2.700561000 & 1.229570000 & 1 & -2.427910000 & 3.834318000 & -0.876241000 & 1 \\
\hline 6 & 5.812478000 & 3.324959000 & -0.778679000 & 6 & -0.955030000 & 5.147834000 & 0.000045000 & 6 \\
\hline 1 & 5.690553000 & 2.985181000 & -1.809185000 & 1 & -0.314726000 & 5.209697000 & 0.881404000 & 1 \\
\hline 1 & 5.367746000 & 4.319536000 & -0.705265000 & 1 & -0.314519000 & 5.209722000 & -0.881162000 & 1 \\
\hline 1 & 6.881362000 & 3.426387000 & -0.588595000 & 1 & -1.605804000 & 6.021796000 & -0.000019000 & 1 \\
\hline 7 & 2.962663000 & 1.265249000 & 0.875063000 & 7 & -1.626376000 & 1.347549000 & -0.000021000 & 7 \\
\hline 1 & 3.074510000 & 1.565258000 & 1.837110000 & 1 & -2.216012000 & 1.222872000 & -0.817723000 & 1 \\
\hline 1 & 3.362868000 & 0.335388000 & 0.815424000 & 1 & -2.216049000 & 1.222841000 & 0.817650000 & 1 \\
\hline 1 & 0.684608000 & 0.534651000 & 0.438255000 & 1 & -0.307180000 & -0.242390000 & 2.154807000 & 1 \\
\hline 6 & -3.788787000 & 1.728248000 & -0.011964000 & 6 & 3.803349000 & -0.425849000 & 0.000005000 & 6 \\
\hline 9 & -4.319262000 & 1.836958000 & 1.228301000 & 9 & 4.361239000 & 0.815750000 & -0.000202000 & 9 \\
\hline
\end{tabular}




\begin{tabular}{|c|c|c|c|c|c|c|c|c|c|c|c|}
\hline $\begin{array}{l}9 \\
9\end{array}$ & $\begin{array}{l}-3.380687000 \\
-4.801636000\end{array}$ & $\begin{array}{l}2.963470000 \\
1.390821000\end{array}$ & $\begin{array}{l}-0.366086000 \\
-0.835868000\end{array}$ & $\begin{array}{l}9 \\
9\end{array}$ & $\begin{array}{l}4.299664000 \\
4.299632000\end{array}$ & $\begin{array}{l}-1.055339000 \\
-1.055691000\end{array}$ & $\begin{array}{r}1.087444000 \\
-1.087250000\end{array}$ & $\begin{array}{l}9 \\
9\end{array}$ & $\begin{array}{l}-4.708161000 \\
-5.296896000\end{array}$ & $\begin{array}{l}-1.582558000 \\
-0.006064000\end{array}$ & $\begin{array}{r}-0.485025000 \\
0.886127000\end{array}$ \\
\hline \multicolumn{12}{|c|}{$n=4, \mathrm{R}=-\mathrm{COOH}$} \\
\hline 6 & -2.896958000 & 1.165892000 & 0.018768000 & 6 & -2.548870000 & -0.525411000 & 0.037093000 & 6 & 3.405850000 & 0.011830000 & -0.008029000 \\
\hline 6 & -1.548486000 & 1.451306000 & -0.197955000 & 6 & -1.846019000 & -0.453163000 & -1.172037000 & 6 & 2.408216000 & -0.621364000 & 0.733575000 \\
\hline 6 & -3.295607000 & -0.154331000 & 0.231835000 & 6 & -1.820689000 & -0.437311000 & 1.230367000 & 6 & 3.062075000 & 0.982839000 & -0.948502000 \\
\hline 1 & -1.235515000 & 2.471484000 & -0.363144000 & 1 & -2.382843000 & -0.540083000 & -2.105840000 & 1 & 2.673974000 & -1.384329000 & 1.449090000 \\
\hline 1 & -4.342474000 & -0.364181000 & 0.398801000 & 1 & -2.354178000 & -0.512650000 & 2.168123000 & 1 & 3.846100000 & 1.457829000 & -1.519780000 \\
\hline 6 & -0.600735000 & 0.435308000 & -0.203093000 & 6 & -0.475143000 & -0.289464000 & -1.200256000 & 6 & 1.074684000 & -0.285732000 & 0.547298000 \\
\hline 6 & -2.361843000 & -1.178600000 & 0.229227000 & 6 & -0.451342000 & -0.276080000 & 1.232106000 & 6 & 1.733647000 & 1.324388000 & -1.139684000 \\
\hline 1 & -2.673686000 & -2.199231000 & 0.394460000 & 1 & 0.089765000 & -0.250668000 & 2.167291000 & 1 & 1.464952000 & 2.076688000 & -1.870693000 \\
\hline 6 & -1.022513000 & -0.871088000 & 0.011474000 & 6 & 0.243313000 & -0.206217000 & 0.008024000 & 6 & 0.753812000 & 0.689823000 & -0.386374000 \\
\hline 53 & 0.404707000 & -2.442799000 & 0.008910000 & 53 & 2.281319000 & -1.501845000 & -0.006170000 & 53 & -2.624920000 & -1.509095000 & -0.113515000 \\
\hline 6 & 3.725005000 & 1.939883000 & 0.003310000 & 6 & 0.447350000 & 2.612542000 & -0.004699000 & 6 & -1.203143000 & 2.023361000 & 0.430494000 \\
\hline 1 & 3.483239000 & 2.927838000 & -0.397591000 & 1 & -0.204253000 & 2.554073000 & -0.877311000 & 1 & -1.304638000 & 1.464096000 & 1.359177000 \\
\hline 1 & 3.438375000 & 1.961757000 & 1.058001000 & 1 & -0.193267000 & 2.557882000 & 0.876240000 & 1 & -0.468044000 & 2.815518000 & 0.570703000 \\
\hline 6 & 5.233306000 & 1.700322000 & -0.108770000 & 6 & 1.249878000 & 3.912479000 & -0.012508000 & 6 & -2.548047000 & 2.565819000 & -0.028494000 \\
\hline 1 & 5.511928000 & 1.676422000 & -1.166991000 & 1 & 1.898594000 & 3.932984000 & -0.892564000 & 1 & -3.219548000 & 1.725466000 & -0.212007000 \\
\hline 1 & 5.465419000 & 0.708726000 & 0.291985000 & 1 & 1.908865000 & 3.936884000 & 0.859757000 & 1 & -2.419696000 & 3.097132000 & -0.977465000 \\
\hline 6 & 6.061156000 & 2.758448000 & 0.619369000 & 6 & 0.343178000 & 5.142991000 & -0.009767000 & 6 & -3.153853000 & 3.507334000 & 1.011040000 \\
\hline 1 & 5.871068000 & 3.755363000 & 0.216339000 & 1 & -0.305098000 & 5.158189000 & -0.887333000 & 1 & -3.337869000 & 2.985232000 & 1.950681000 \\
\hline 1 & 5.821966000 & 2.782955000 & 1.684527000 & 1 & -0.294798000 & 5.161832000 & 0.875207000 & 1 & -2.499092000 & 4.356147000 & 1.217830000 \\
\hline 1 & 7.129717000 & 2.562603000 & 0.525339000 & 1 & 0.931554000 & 6.060108000 & -0.015137000 & 1 & -4.106949000 & 3.900580000 & 0.659953000 \\
\hline 7 & 2.866392000 & 0.962417000 & -0.671114000 & 7 & 1.276500000 & 1.398635000 & -0.007280000 & 7 & -0.646492000 & 1.048542000 & -0.575928000 \\
\hline 1 & 3.034374000 & 0.029545000 & -0.310567000 & 1 & 1.879452000 & 1.320310000 & 0.806538000 & 1 & -0.781407000 & 1.422918000 & -1.513266000 \\
\hline 1 & 3.075839000 & 0.928791000 & -1.662536000 & 1 & 1.866614000 & 1.314522000 & -0.829852000 & 1 & -1.289795000 & 0.135968000 & -0.509093000 \\
\hline 1 & 0.443594000 & 0.668440000 & -0.370239000 & 1 & 0.047496000 & -0.274760000 & -2.146084000 & 1 & 0.288655000 & -0.794787000 & 1.088098000 \\
\hline 6 & -3.939462000 & 2.221650000 & 0.033121000 & 6 & -4.002934000 & -0.699906000 & 0.106505000 & 6 & 4.848744000 & -0.314659000 & 0.157783000 \\
\hline 8 & -5.117042000 & 2.028724000 & 0.216972000 & 8 & -4.658758000 & -0.761927000 & 1.123623000 & 8 & 5.742609000 & 0.206295000 & -0.461867000 \\
\hline 8 & -3.441036000 & 3.463834000 & -0.184782000 & 8 & -4.594598000 & -0.786938000 & -1.119236000 & 8 & 5.067785000 & -1.269302000 & 1.089269000 \\
\hline 1 & -4.196633000 & 4.067729000 & -0.154033000 & 1 & -5.538800000 & -0.903134000 & -0.945128000 & 1 & 6.024516000 & -1.414023000 & 1.120696000 \\
\hline \multicolumn{12}{|c|}{$n=4, \mathrm{R}=-\mathrm{CN}$} \\
\hline 6 & 0.588683000 & 1.623443000 & 0.007722000 & 6 & -2.523896000 & -1.469683000 & -0.000002000 & 6 & 3.739241000 & -0.147807000 & -0.076485000 \\
\hline 6 & -0.586559000 & 2.310185000 & -0.311680000 & 6 & -1.871530000 & -1.164640000 & -1.203544000 & 6 & 3.432597000 & 0.755030000 & 0.946347000 \\
\hline 6 & 0.544316000 & 0.258973000 & 0.314185000 & 6 & -1.871526000 & -1.164654000 & 1.203542000 & 6 & 2.718113000 & -0.674257000 & -0.873980000 \\
\hline 1 & -0.547782000 & 3.364129000 & -0.548125000 & 1 & -2.346107000 & -1.411652000 & -2.143544000 & 1 & 4.222023000 & 1.153114000 & 1.566682000 \\
\hline 1 & 1.464466000 & -0.259398000 & 0.559250000 & 1 & -2.346098000 & -1.411677000 & 2.143541000 & 1 & 2.957575000 & -1.385215000 & -1.651065000 \\
\hline 6 & -1.800194000 & 1.640475000 & -0.325141000 & 6 & -0.626158000 & -0.570559000 & -1.214755000 & 6 & 2.117055000 & 1.133634000 & 1.158390000 \\
\hline 6 & -0.671519000 & -0.409059000 & 0.300254000 & 6 & -0.626154000 & -0.570573000 & 1.214756000 & 6 & 1.401812000 & -0.297691000 & -0.660303000 \\
\hline 1 & -0.707308000 & -1.462145000 & 0.536899000 & 1 & -0.131407000 & -0.382106000 & 2.156793000 & 1 & 0.598669000 & -0.727799000 & -1.242828000 \\
\hline 6 & -1.834252000 & 0.284104000 & -0.018549000 & 6 & 0.020643000 & -0.267562000 & 0.000001000 & 6 & 1.115739000 & 0.609798000 & 0.351563000 \\
\hline 53 & -3.688343000 & -0.737971000 & -0.037411000 & 53 & 2.363022000 & -0.840120000 & -0.000002000 & 53 & -2.264893000 & -1.515757000 & 0.061547000 \\
\hline 6 & 4.802737000 & -1.100232000 & -0.007815000 & 6 & -0.700010000 & 2.461777000 & 0.000017000 & 6 & -0.761392000 & 2.117261000 & -0.331494000 \\
\hline 1 & 4.626630000 & -2.172539000 & 0.114478000 & 1 & -1.293316000 & 2.200574000 & -0.876940000 & 1 & -0.014419000 & 2.911197000 & -0.328328000 \\
\hline 1 & 4.488445000 & -0.853711000 & -1.025354000 & 1 & -1.293281000 & 2.200618000 & 0.877011000 & 1 & -0.814262000 & 1.691947000 & -1.332668000 \\
\hline 6 & 6.298227000 & -0.809033000 & 0.145410000 & 6 & -0.358488000 & 3.950904000 & -0.000027000 & 6 & -2.124746000 & 2.614599000 & 0.125113000 \\
\hline 1 & 6.604560000 & -1.053754000 & 1.167493000 & 1 & 0.252899000 & 4.182927000 & -0.876352000 & 1 & -2.041260000 & 3.019243000 & 1.139034000 \\
\hline 1 & 6.463192000 & 0.265518000 & 0.024163000 & 1 & 0.252979000 & 4.182963000 & 0.876232000 & 1 & -2.811079000 & 1.767473000 & 0.170679000 \\
\hline 6 & 7.161462000 & -1.581519000 & -0.851134000 & 6 & -1.613784000 & 4.823141000 & 0.000010000 & 6 & -2.669969000 & 3.688690000 & -0.814671000 \\
\hline 1 & 7.031882000 & -2.659697000 & -0.734628000 & 1 & -2.227842000 & 4.632020000 & -0.881349000 & 1 & -2.001034000 & 4.549286000 & -0.876307000 \\
\hline 1 & 6.899121000 & -1.327601000 & -1.880219000 & 1 & -2.227753000 & 4.632077000 & 0.881443000 & 1 & -2.806974000 & 3.294690000 & -1.822432000 \\
\hline 1 & 8.220619000 & -1.359660000 & -0.716583000 & 1 & -1.351994000 & 5.880776000 & -0.000037000 & 1 & -3.638132000 & 4.044818000 & -0.465495000 \\
\hline 7 & 3.914222000 & -0.389130000 & 0.916689000 & 7 & 0.476779000 & 1.579107000 & 0.000015000 & 7 & -0.268263000 & 1.008772000 & 0.562174000 \\
\hline 1 & 4.025834000 & 0.613838000 & 0.809367000 & 1 & 1.067920000 & 1.696717000 & 0.817990000 & 1 & -0.951230000 & 0.129045000 & 0.385889000 \\
\hline 1 & 4.160651000 & -0.605529000 & 1.876249000 & 1 & 1.067923000 & 1.696727000 & -0.817957000 & 1 & -0.410495000 & 1.272161000 & 1.535527000 \\
\hline 1 & -2.706857000 & 2.172241000 & -0.571791000 & 1 & -0.131413000 & -0.382083000 & -2.156792000 & 1 & 1.877375000 & 1.831733000 & 1.950152000 \\
\hline 6 & 1.840815000 & 2.311965000 & 0.023855000 & 6 & -3.808685000 & -2.077733000 & -0.000003000 & 6 & 5.097398000 & -0.533764000 & -0.300840000 \\
\hline 7 & 2.856692000 & 2.856982000 & 0.041728000 & 7 & -4.856085000 & -2.563966000 & -0.000004000 & 7 & 6.193616000 & -0.838704000 & -0.481127000 \\
\hline
\end{tabular}


Table S13. Coordinates of the optimized reactants, products and transition states for the 4$\mathrm{MePhBr}+\mathrm{NH}_{2} \mathrm{R}$ amination for $\mathrm{R}=-\mathrm{Me},-\mathrm{Et}$, $-{ }^{\mathrm{n}} \mathrm{Pr}$, and $-{ }^{\mathrm{n}} \mathrm{Bu}$

\begin{tabular}{|c|c|c|c|c|c|c|c|c|c|c|c|}
\hline \multicolumn{4}{|c|}{ Reactant } & \multicolumn{4}{|c|}{ Transition State } & & & roduct & \\
\hline & & & & & & $=-\mathbf{M e}$ & & & & & \\
\hline 6 & 2.790084000 & 0.905883000 & 0.002614000 & 6 & 2.632270000 & -0.412529000 & -0.000732000 & 6 & 3.192260000 & $\begin{array}{l}-0.498617000 \\
\end{array}$ & 0.007462000 \\
\hline 6 & 1.532312000 & 1.496456000 & 0.121552000 & 6 & 1.917195000 & -0.266327000 & 1.188387000 & 6 & 2.074188000 & -1.127056000 & 0.562464000 \\
\hline 6 & 2.852255000 & -0.483180000 & -0.111828000 & 6 & 1.917729000 & -0.264595000 & -1.189131000 & 6 & 3.012277000 & 0.736111000 & -0.613754000 \\
\hline 1 & 1.454427000 & 2.572402000 & 0.218192000 & 1 & 2.425030000 & -0.395248000 & 2.137284000 & 1 & 2.186221000 & -2.093627000 & 1.036765000 \\
\hline 1 & 3.814999000 & -0.971519000 & -0.199178000 & 1 & 2.425874000 & -0.391944000 & -2.137986000 & 1 & 3.860111000 & 1.240483000 & -1.058402000 \\
\hline 6 & 0.364649000 & 0.740428000 & 0.122453000 & 6 & 0.558066000 & 0.021693000 & 1.208216000 & 6 & 0.815132000 & -0.548982000 & 0.506241000 \\
\hline 6 & 1.700969000 & -1.261137000 & -0.113858000 & 6 & 0.557904000 & 0.023485000 & -1.208960000 & 6 & 1.758065000 & 1.332276000 & -0.674804000 \\
\hline 1 & 1.765142000 & -2.335952000 & -0.200953000 & 1 & 0.030057000 & 0.085973000 & -2.149957000 & 1 & 1.637019000 & 2.291703000 & -1.163056000 \\
\hline 6 & 0.468144000 & -0.636716000 & 0.002411000 & 6 & -0.126982000 & 0.153376000 & -0.000580000 & 6 & 0.669232000 & 0.686175000 & -0.111893000 \\
\hline 35 & -1.123413000 & -1.712046000 & 0.003080000 & 35 & -2.134478000 & -0.912180000 & -0.000151000 & 35 & -2.929494000 & -0.691581000 & -0.172957000 \\
\hline 6 & -3.857760000 & 2.509889000 & -0.475567000 & 6 & -0.269046000 & 2.971668000 & 0.000654000 & 6 & -1.012992000 & 2.138958000 & 1.011107000 \\
\hline 1 & -3.707328000 & 3.582805000 & -0.352337000 & 1 & 0.366299000 & 2.938106000 & 0.883482000 & 1 & -0.970643000 & 1.514373000 & 1.897975000 \\
\hline 1 & -3.561505000 & 2.255916000 & -1.493437000 & 1 & 0.366744000 & 2.936844000 & -0.881825000 & 1 & -0.319596000 & 2.971757000 & 1.103498000 \\
\hline 1 & -4.931316000 & 2.306393000 & -0.376386000 & 1 & -0.840216000 & 3.900647000 & -0.000136000 & 1 & -2.032471000 & 2.490814000 & 0.875605000 \\
\hline 7 & -3.001972000 & 1.788922000 & 0.469416000 & 7 & -1.134079000 & 1.791715000 & 0.001142000 & 7 & -0.656103000 & 1.295315000 & -0.163795000 \\
\hline 1 & -3.116502000 & 0.787703000 & 0.364714000 & 1 & -1.729365000 & 1.713132000 & -0.816064000 & 1 & -0.748962000 & 1.841924000 & -1.016365000 \\
\hline 1 & -3.257355000 & 2.011131000 & 1.423829000 & 1 & -1.728289000 & 1.712896000 & 0.819111000 & 1 & -1.475406000 & 0.495142000 & -0.229953000 \\
\hline 1 & -0.601298000 & 1.220236000 & 0.219347000 & 1 & 0.029807000 & 0.083174000 & 2.149059000 & 1 & -0.053110000 & -1.056561000 & 0.904726000 \\
\hline 6 & 4.042487000 & 1.742271000 & -0.026940000 & 6 & 4.110837000 & -0.703457000 & 0.000655000 & 6 & 4.548227000 & -1.149361000 & 0.074165000 \\
\hline 1 & 4.883153000 & 1.217091000 & 0.426816000 & 1 & 4.414624000 & -1.216527000 & -0.912250000 & 1 & 5.309734000 & -0.534309000 & -0.402681000 \\
\hline 1 & 4.327711000 & 1.985971000 & -1.053716000 & 1 & 4.705353000 & 0.212258000 & 0.070910000 & 1 & 4.852279000 & -1.319337000 & 1.108679000 \\
\hline 1 & 3.904312000 & 2.683614000 & 0.504387000 & 1 & 4.391524000 & -1.334909000 & 0.844622000 & 1 & 4.541487000 & -2.121261000 & -0.422660000 \\
\hline & & & & & & $=-\mathbf{E} \mathbf{t}$ & & & & & \\
\hline 6 & 2.995139000 & 1.351138000 & 0.023663000 & 6 & 2.816401000 & -0.037713000 & 0.000382000 & 6 & 3.400643000 & -0.381470000 & -0.071408000 \\
\hline 6 & 3.336270000 & 0.001636000 & 0.109164000 & 6 & 2.087605000 & -0.069429000 & 1.189081000 & 6 & 3.127436000 & 0.771480000 & 0.663342000 \\
\hline 6 & 1.642143000 & 1.679164000 & -0.066815000 & 6 & 2.087646000 & -0.070511000 & -1.188715000 & 6 & 2.329127000 & -1.057135000 & -0.660309000 \\
\hline 1 & 4.377943000 & -0.284047000 & 0.187233000 & 1 & 2.611646000 & -0.057791000 & 2.137851000 & 1 & 3.938528000 & 1.311596000 & 1.133923000 \\
\hline 1 & 1.347394000 & 2.719659000 & -0.127955000 & 1 & 2.611789000 & -0.059792000 & -2.137476000 & 1 & 2.514878000 & -1.960546000 & -1.226934000 \\
\hline 6 & 2.364847000 & -0.992553000 & 0.099938000 & 6 & 0.699168000 & -0.130627000 & 1.208611000 & 6 & 1.827001000 & 1.240302000 & 0.803827000 \\
\hline 6 & 0.651210000 & 0.703802000 & -0.078992000 & 6 & 0.699551000 & -0.131706000 & -1.208321000 & 6 & 1.024205000 & -0.606480000 & -0.525632000 \\
\hline 1 & -0.392726000 & 0.981385000 & -0.151432000 & 1 & 0.171492000 & -0.192188000 & -2.149350000 & 1 & 0.194709000 & -1.153969000 & -0.952675000 \\
\hline 6 & 1.030939000 & -0.627362000 & 0.004171000 & 6 & 0.003830000 & -0.176091000 & 0.000229000 & 6 & 0.784697000 & 0.548190000 & 0.207286000 \\
\hline 35 & -0.312499000 & -2.000003000 & -0.006172000 & 35 & -1.680104000 & -1.703129000 & 0.000141000 & 35 & -2.608499000 & -1.231538000 & 0.167690000 \\
\hline 6 & -3.943212000 & 1.724368000 & 0.314320000 & 6 & -0.851786000 & 2.536359000 & -0.000870000 & 6 & -1.048220000 & 1.947378000 & -0.762429000 \\
\hline 1 & -3.674129000 & 1.690905000 & 1.372211000 & 1 & -0.208138000 & 2.626584000 & 0.874368000 & 1 & -0.273608000 & 2.696363000 & -0.923395000 \\
\hline 1 & -3.935420000 & 2.779479000 & 0.031619000 & 1 & -0.209530000 & 2.627126000 & -0.877060000 & 1 & -1.121540000 & 1.323288000 & -1.650697000 \\
\hline 6 & -5.348128000 & 1.151866000 & 0.122490000 & 6 & -1.928692000 & 3.615335000 & 0.000333000 & 6 & -2.388815000 & 2.569959000 & -0.417936000 \\
\hline 1 & -5.389207000 & 0.105112000 & 0.431086000 & 1 & -2.564594000 & 3.543171000 & 0.884047000 & 1 & -2.319253000 & 3.201881000 & 0.470390000 \\
\hline 1 & -5.651835000 & 1.203372000 & -0.925457000 & 1 & -2.566053000 & 3.543778000 & -0.882376000 & 1 & -3.137111000 & 1.797249000 & -0.247246000 \\
\hline 1 & -6.084309000 & 1.703966000 & 0.711334000 & 1 & -1.468304000 & 4.604257000 & 0.000305000 & 1 & -2.719171000 & 3.197884000 & -1.244836000 \\
\hline 7 & -2.875748000 & 1.056012000 & -0.434426000 & 7 & -1.380319000 & 1.168059000 & -0.000784000 & 7 & -0.585680000 & 1.033882000 & 0.339664000 \\
\hline 1 & -3.069054000 & 1.081546000 & -1.429408000 & 1 & -1.937693000 & 0.941331000 & -0.818053000 & 1 & -1.310185000 & 0.165486000 & 0.352087000 \\
\hline 1 & -2.816284000 & 0.076378000 & -0.178600000 & 1 & -1.937973000 & 0.941648000 & 0.816383000 & 1 & -0.696664000 & 1.507188000 & 1.233925000 \\
\hline 1 & 2.644935000 & -2.033459000 & 0.169903000 & 1 & 0.171121000 & -0.190358000 & 2.149690000 & 1 & 1.633718000 & 2.137963000 & 1.378716000 \\
\hline 6 & 4.055542000 & 2.420716000 & 0.003685000 & 6 & 4.323236000 & -0.013440000 & -0.000179000 & 6 & 4.806407000 & -0.902470000 & -0.209411000 \\
\hline 1 & 3.709982000 & 3.332552000 & 0.490976000 & 1 & 4.713258000 & 0.526204000 & -0.864264000 & 1 & 5.038222000 & -1.145221000 & -1.247193000 \\
\hline 1 & 4.963810000 & 2.090611000 & 0.507431000 & 1 & 4.713751000 & 0.468780000 & 0.896740000 & 1 & 5.537570000 & -0.174830000 & 0.139779000 \\
\hline 1 & 4.328118000 & 2.683910000 & -1.021699000 & 1 & 4.743351000 & -1.022713000 & -0.033560000 & 1 & 4.942255000 & -1.816217000 & 0.373395000 \\
\hline & & & & & & & & & & & \\
\hline 6 & -3.232672000 & -1.636480000 & -0.055423000 & 6 & -3.087301000 & -0.994396000 & -0.077922000 & 6 & 3.654372000 & -0.050638000 & -0.160832000 \\
\hline 6 & -1.860311000 & -1.774076000 & 0.152376000 & 6 & -2.703201000 & -0.315929000 & 1.079979000 & 6 & 3.248840000 & 0.934406000 & 0.739396000 \\
\hline 6 & -3.739044000 & -0.351282000 & -0.249822000 & 6 & -2.146271000 & -1.079231000 & -1.102138000 & 6 & 2.668477000 & -0.807665000 & -0.797193000 \\
\hline 1 & -1.438428000 & -2.760755000 & 0.299407000 & 1 & -3.405034000 & -0.215588000 & 1.899863000 & 1 & 3.992430000 & 1.526440000 & 1.256838000 \\
\hline 1 & -4.799587000 & -0.212987000 & -0.420286000 & 1 & -2.405235000 & -1.584499000 & -2.025330000 & 1 & 2.958991000 & -1.591180000 & -1.485120000 \\
\hline 6 & -1.008602000 & -0.674671000 & 0.173516000 & 6 & -1.445066000 & 0.254337000 & 1.221952000 & 6 & 1.902163000 & 1.165871000 & 0.989193000 \\
\hline 6 & -2.909481000 & 0.763445000 & -0.233383000 & 6 & -0.875798000 & -0.521493000 & -0.995672000 & 6 & 1.318829000 & -0.593356000 & -0.555894000 \\
\hline 1 & 0.054125000 & -0.805336000 & 0.334718000 & 1 & -1.193620000 & 0.801896000 & 2.119176000 & 1 & 1.605885000 & 1.933253000 & 1.694185000 \\
\hline 1 & -3.316109000 & 1.752156000 & -0.388375000 & 1 & -0.190642000 & -0.574174000 & -1.828264000 & 1 & 0.560623000 & -1.207853000 & -1.022497000 \\
\hline 6 & -1.550762000 & 0.586615000 & -0.019933000 & 6 & -0.534413000 & 0.159239000 & 0.168946000 & 6 & 0.946433000 & 0.401192000 & 0.337673000 \\
\hline 35 & -0.403065000 & 2.126257000 & -0.001868000 & 35 & 0.538245000 & 2.148172000 & -0.238307000 & 35 & -2.142111000 & -1.862551000 & 0.109581000 \\
\hline 6 & -4.144592000 & -2.835320000 & -0.045590000 & 6 & -4.471935000 & -1.570149000 & -0.227372000 & 6 & 5.112939000 & -0.287036000 & -0.449541000 \\
\hline 1 & -5.005248000 & -2.686141000 & -0.697649000 & 1 & -4.492834000 & -2.364482000 & -0.973990000 & 1 & 5.406117000 & 0.186569000 & -1.389670000 \\
\hline 1 & -3.622922000 & -3.734374000 & -0.373220000 & 1 & -4.835454000 & -1.987815000 & 0.712823000 & 1 & 5.746486000 & 0.122011000 & 0.336365000 \\
\hline 1 & -4.527744000 & -3.029047000 & 0.959741000 & 1 & -5.192653000 & -0.810171000 & -0.542160000 & 1 & 5.330398000 & -1.351186000 & -0.542481000 \\
\hline 6 & 3.635217000 & -1.144599000 & -0.024284000 & 6 & 2.110916000 & -1.024417000 & -0.060264000 & 6 & -1.130990000 & 1.620974000 & -0.337863000 \\
\hline 1 & 3.725432000 & -2.196241000 & 0.261472000 & 1 & 1.611447000 & -1.826690000 & -0.605683000 & 1 & -0.484448000 & 2.495238000 & -0.417960000 \\
\hline 1 & 3.373279000 & -1.140254000 & -1.085620000 & 1 & 2.327068000 & -0.224013000 & -0.767712000 & 1 & -1.177469000 & 1.129688000 & -1.308561000 \\
\hline 6 & 4.986404000 & -0.449383000 & 0.168459000 & 6 & 3.399616000 & -1.538050000 & 0.581421000 & 6 & -2.523148000 & 1.986011000 & 0.155261000 \\
\hline 1 & 5.238410000 & -0.452684000 & 1.233637000 & 1 & 3.156425000 & -2.308875000 & 1.318486000 & 1 & -2.446334000 & 2.456239000 & 1.141448000 \\
\hline 1 & 4.889165000 & 0.601619000 & -0.120442000 & 1 & 3.878217000 & -0.721844000 & 1.128922000 & 1 & -3.101399000 & 1.068894000 & 0.276440000 \\
\hline 6 & 6.112889000 & -1.103219000 & -0.630755000 & 6 & 4.370321000 & -2.103191000 & -0.455311000 & 6 & -3.230585000 & 2.933637000 & -0.811824000 \\
\hline 1 & 6.249048000 & -2.147107000 & -0.340341000 & 1 & 3.927163000 & -2.940801000 & -0.996821000 & 1 & -2.672315000 & 3.861955000 & -0.949334000 \\
\hline 1 & 5.899283000 & -1.084962000 & -1.701490000 & 1 & 4.650590000 & -1.344149000 & -1.187234000 & 1 & -3.358297000 & 2.469307000 & -1.790527000 \\
\hline 1 & 7.062635000 & -0.590514000 & -0.474830000 & 1 & 5.283920000 & -2.460814000 & 0.019206000 & 1 & -4.220429000 & 3.193420000 & -0.438487000 \\
\hline 7 & 2.506284000 & -0.575982000 & 0.714968000 & 7 & 1.142910000 & -0.483568000 & 0.901642000 & 7 & -0.473021000 & 0.636720000 & 0.588502000 \\
\hline 1 & 2.353270000 & 0.391323000 & 0.451823000 & 1 & 1.498819000 & 0.339278000 & 1.378153000 & 1 & -1.054631000 & -0.325470000 & 0.484519000 \\
\hline 1 & 2.694404000 & -0.578003000 & 1.711106000 & 1 & 0.841656000 & -1.178623000 & 1.578106000 & 1 & -0.604570000 & 0.944544000 & 1.549750000 \\
\hline & & & & & & $=-\mathrm{n} \mathrm{Bu}$ & & & & & \\
\hline 6 & -3.440739000 & 1.945255000 & -0.010275000 & 6 & 2.046483000 & 2.397653000 & -0.001187000 & 6 & 3.937778000 & -0.490339000 & -0.185214000 \\
\hline 6 & -2.053876000 & 1.849996000 & 0.105224000 & 6 & 1.749377000 & 1.730737000 & 1.188107000 & 6 & 3.107393000 & 0.437137000 & -0.819247000 \\
\hline 6 & -4.170263000 & 0.761973000 & -0.125143000 & 6 & 1.753460000 & 1.730501000 & -1.189787000 & 6 & 3.361469000 & -1.376505000 & 0.723685000 \\
\hline
\end{tabular}




\begin{tabular}{|c|c|c|c|c|c|c|c|c|c|c|c|}
\hline 1 & -1.462609000 & 2.752296000 & 0.202344000 & 1 & 1.971331000 & 2.205100000 & 2.137211000 & 1 & 3.534138000 & 1.142672000 & -1.520580000 \\
\hline 1 & -5.249181000 & 0.803566000 & -0.209509000 & 1 & 1.978536000 & 2.204331000 & -2.138276000 & 1 & 3.983168000 & -2.101154000 & 1.232930000 \\
\hline 6 & -1.401243000 & 0.621965000 & 0.102024000 & 6 & 1.187636000 & 0.460511000 & 1.207085000 & 6 & 1.745282000 & 0.487146000 & -0.561949000 \\
\hline 6 & -3.542004000 & -0.477760000 & -0.130910000 & 6 & 1.190952000 & 0.459165000 & -1.210011000 & 6 & 1.998021000 & -1.343528000 & 0.990160000 \\
\hline 1 & -4.120812000 & -1.385584000 & -0.218487000 & 1 & 1.012171000 & -0.040759000 & -2.151331000 & 1 & 1.567183000 & -2.039229000 & 1.700065000 \\
\hline 6 & -2.161005000 & -0.531684000 & -0.018152000 & 6 & 0.920342000 & -0.182854000 & -0.002143000 & 6 & 1.199905000 & -0.412480000 & 0.344212000 \\
\hline 35 & -1.289596000 & -2.242405000 & -0.021902000 & 35 & 1.540304000 & -2.369862000 & 0.001520000 & 35 & -1.416663000 & 2.388115000 & 0.106662000 \\
\hline 6 & 3.294887000 & 0.890247000 & 0.019744000 & 6 & -1.893757000 & 0.253730000 & 0.000311000 & 6 & -1.075920000 & -1.234332000 & -0.294037000 \\
\hline 1 & 3.151009000 & 1.210712000 & -1.015361000 & 1 & -1.692285000 & 0.868115000 & 0.879075000 & 1 & -1.040160000 & -0.760291000 & -1.273576000 \\
\hline 1 & 3.313574000 & 1.803855000 & 0.619955000 & 1 & -1.690475000 & 0.874827000 & -0.873324000 & 1 & -0.602989000 & -2.214278000 & -0.361599000 \\
\hline 6 & 4.639895000 & 0.169351000 & 0.148248000 & 6 & -3.344269000 & -0.225825000 & -0.002819000 & 6 & -2.505578000 & -1.324970000 & 0.217158000 \\
\hline 1 & 4.614103000 & -0.745019000 & -0.454491000 & 1 & -3.519564000 & -0.862077000 & 0.870304000 & 1 & -2.902010000 & -0.313868000 & 0.329062000 \\
\hline 1 & 4.774257000 & -0.152838000 & 1.186730000 & 1 & -3.518817000 & -0.853104000 & -0.882549000 & 1 & -2.508066000 & -1.788002000 & 1.210794000 \\
\hline 6 & 5.832326000 & 1.027348000 & -0.277233000 & 6 & -4.343186000 & 0.934116000 & 0.002796000 & 6 & -3.399205000 & -2.137196000 & -0.722144000 \\
\hline 1 & 5.692457000 & 1.349258000 & -1.313634000 & 1 & -4.161174000 & 1.560630000 & 0.880477000 & 1 & -3.401667000 & -1.664295000 & -1.707626000 \\
\hline 1 & 5.850100000 & 1.942079000 & 0.323162000 & 1 & -4.160562000 & 1.569803000 & -0.868139000 & 1 & -2.976660000 & -3.137207000 & -0.860550000 \\
\hline 7 & 2.111346000 & 0.122826000 & 0.415239000 & 7 & -0.902296000 & -0.826266000 & -0.002834000 & 7 & -0.236172000 & -0.372984000 & 0.608193000 \\
\hline 1 & 2.188902000 & -0.182878000 & 1.378703000 & 1 & -0.945619000 & -1.423706000 & -0.821907000 & 1 & -0.413456000 & -0.632799000 & 1.576271000 \\
\hline 1 & 2.022575000 & -0.717336000 & -0.145226000 & 1 & -0.945692000 & -1.429017000 & 0.812356000 & 1 & -0.627900000 & 0.678505000 & 0.490396000 \\
\hline 1 & -0.323489000 & 0.572497000 & 0.194498000 & 1 & 1.005370000 & -0.039198000 & 2.147871000 & 1 & 1.112000000 & 1.229774000 & -1.028602000 \\
\hline 6 & -4.127237000 & 3.285797000 & -0.036336000 & 6 & 2.694893000 & 3.757941000 & 0.002346000 & 6 & 5.416638000 & -0.513945000 & -0.466992000 \\
\hline 1 & -5.141455000 & 3.222114000 & 0.357824000 & 1 & 2.521727000 & 4.282307000 & -0.937878000 & 1 & 5.902340000 & -1.357973000 & 0.020378000 \\
\hline 1 & -3.581036000 & 4.023545000 & 0.551425000 & 1 & 2.308127000 & 4.384144000 & 0.807955000 & 1 & 5.613481000 & -0.582914000 & -1.537972000 \\
\hline 1 & -4.197908000 & 3.670873000 & -1.057011000 & 1 & 3.777661000 & 3.688610000 & 0.140107000 & 1 & 5.896975000 & 0.399473000 & -0.110023000 \\
\hline 6 & 7.173544000 & 0.307481000 & -0.145443000 & 6 & -5.797677000 & 0.467714000 & -0.000136000 & 6 & -4.834033000 & -2.254259000 & -0.211097000 \\
\hline 1 & 7.200252000 & -0.593057000 & -0.762364000 & 1 & -6.018535000 & -0.142663000 & 0.877626000 & 1 & -5.289820000 & -1.269912000 & -0.095210000 \\
\hline 1 & 8.001435000 & 0.946308000 & -0.456229000 & 1 & -6.484279000 & 1.314706000 & 0.004136000 & 1 & -5.451798000 & -2.829077000 & -0.901508000 \\
\hline 1 & 7.358563000 & 0.004183000 & 0.887078000 & 1 & -6.018003000 & -0.133332000 & -0.884441000 & 1 & -4.868834000 & -2.752917000 & 0.759614000 \\
\hline
\end{tabular}


Table S14. Coordinates of the optimized reactants, products and transition states for the 4$\mathrm{MePhCl}+\mathrm{NH}_{2}{ }^{\mathrm{n}} \mathrm{Pr}$ amination at different temperatures. The default used for thermochemistry analysis is $25^{\circ} \mathrm{C}$.

\begin{tabular}{|c|c|c|c|c|c|c|c|c|c|c|c|}
\hline \multicolumn{4}{|c|}{ Reactant } & \multicolumn{4}{|c|}{ Transition State } & & & roduct & \\
\hline & & & & & & $=-20^{\circ} \mathrm{C}$ & & & & & \\
\hline 6 & 3.367533000 & -1.118065000 & 0.059452000 & 6 & -2.436483000 & -1.181233000 & $\begin{array}{l}-0.000615000 \\
\end{array}$ & 6 & 3.282763000 & -0.269511000 & -0.100764000 \\
\hline 6 & 2.004576000 & -1.339647000 & -0.144465000 & 6 & -1.915874000 & -0.667496000 & 1.187553000 & 6 & 2.391210000 & -0.761114000 & -1.048534000 \\
\hline 6 & 3.795249000 & 0.195856000 & 0.245357000 & 6 & -1.916683000 & -0.667154000 & -1.188139000 & 6 & 2.743263000 & 0.261332000 & 1.074041000 \\
\hline 1 & 1.643354000 & -2.351026000 & -0.285536000 & 1 & -2.297745000 & -1.025721000 & 2.136821000 & 1 & 2.773463000 & -1.181518000 & -1.970429000 \\
\hline 1 & 4.845877000 & 0.398636000 & 0.411570000 & 1 & -2.299131000 & -1.025055000 & -2.137206000 & 1 & 3.405220000 & 0.651265000 & 1.837624000 \\
\hline 6 & 1.089252000 & -0.294680000 & -0.169110000 & 6 & -0.926734000 & 0.306617000 & 1.207547000 & 6 & 1.014452000 & -0.729959000 & -0.848057000 \\
\hline 6 & 2.899226000 & 1.258338000 & 0.225161000 & 6 & -0.926948000 & 0.307339000 & -1.208425000 & 6 & 1.376511000 & 0.301405000 & 1.286895000 \\
\hline 1 & 3.241211000 & 2.272482000 & 0.372703000 & 1 & -0.579149000 & 0.710378000 & -2.149412000 & 1 & 0.986795000 & 0.726725000 & 2.204093000 \\
\hline 6 & 1.553662000 & 0.999529000 & 0.016441000 & 6 & -0.433473000 & 0.812585000 & -0.000697000 & 6 & 0.490861000 & -0.195946000 & 0.326628000 \\
\hline 17 & 0.415878000 & 2.343087000 & -0.006396000 & 17 & -0.259119000 & 2.899497000 & 0.000801000 & 17 & -1.875043000 & 2.642669000 & -0.460417000 \\
\hline 6 & -3.565986000 & -0.831800000 & 0.030030000 & 6 & 1.989664000 & -0.587875000 & 0.000681000 & 6 & -1.817455000 & -1.030864000 & -0.111291000 \\
\hline 1 & -3.661808000 & -1.882074000 & -0.258933000 & 1 & 1.585787000 & -1.094616000 & 0.878102000 & 1 & -1.874416000 & -0.766745000 & -1.168915000 \\
\hline 1 & -3.311021000 & -0.832066000 & 1.093086000 & 1 & 1.583560000 & -1.096758000 & -0.874484000 & 1 & -1.426551000 & -2.053154000 & -0.050956000 \\
\hline 6 & -4.910978000 & -0.126605000 & -0.169669000 & 6 & 3.515907000 & -0.636444000 & -0.001137000 & 6 & -3.210871000 & -0.962608000 & 0.503284000 \\
\hline 1 & -5.156118000 & -0.125590000 & -1.236455000 & 1 & 3.896625000 & -0.101645000 & 0.873439000 & 1 & -3.577119000 & 0.064143000 & 0.444663000 \\
\hline 1 & -4.808324000 & 0.923021000 & 0.122377000 & 1 & 3.894418000 & -0.104475000 & -0.878393000 & 1 & -3.146010000 & -1.215496000 & 1.566707000 \\
\hline 6 & -6.047207000 & -0.774487000 & 0.620561000 & 6 & 4.044721000 & -2.070587000 & 0.000544000 & 6 & -4.194485000 & -1.906290000 & -0.185272000 \\
\hline 1 & -6.188460000 & -1.816798000 & 0.326940000 & 1 & 3.705997000 & -2.615789000 & 0.883121000 & 1 & -4.307097000 & -1.655435000 & -1.241328000 \\
\hline 1 & -5.840676000 & -0.760018000 & 1.692748000 & 1 & 3.703720000 & -2.618729000 & -0.879328000 & 1 & -3.862504000 & -2.944330000 & -0.122278000 \\
\hline 1 & -6.992397000 & -0.255014000 & 0.459426000 & 1 & 5.134390000 & -2.084994000 & -0.000833000 & 1 & -5.180344000 & -1.845083000 & 0.275234000 \\
\hline 7 & -2.428127000 & -0.269611000 & -0.700223000 & 7 & 1.436534000 & 0.771865000 & -0.000353000 & 7 & -0.905845000 & -0.088060000 & 0.553768000 \\
\hline 1 & -2.269519000 & 0.695478000 & -0.432503000 & 1 & 1.693402000 & 1.316914000 & -0.817279000 & 1 & -1.100173000 & -0.043199000 & 1.546228000 \\
\hline 1 & -2.610061000 & -0.266631000 & -1.697502000 & 1 & 1.693878000 & 1.318563000 & 0.815323000 & 1 & -1.413190000 & 1.464566000 & 0.009354000 \\
\hline 1 & 0.034646000 & -0.482939000 & -0.326688000 & 1 & -0.578127000 & 0.709384000 & 2.148365000 & 1 & 0.360370000 & -1.117942000 & -1.614920000 \\
\hline 6 & 4.346514000 & -2.262964000 & 0.056165000 & 6 & -3.540851000 & -2.206765000 & 0.000671000 & 6 & 4.771313000 & -0.291608000 & -0.329843000 \\
\hline 1 & 4.593224000 & -2.566909000 & -0.964375000 & 1 & -4.527692000 & -1.740617000 & 0.075250000 & 1 & 5.164402000 & 0.716596000 & -0.481462000 \\
\hline 1 & 5.278559000 & -1.991092000 & 0.550762000 & 1 & -3.533343000 & -2.800041000 & -0.914564000 & 1 & 5.300652000 & -0.717767000 & 0.524313000 \\
\hline 1 & 3.937425000 & -3.137519000 & 0.563139000 & 1 & -3.446794000 & -2.895215000 & 0.842044000 & 1 & 5.027786000 & -0.880614000 & -1.209743000 \\
\hline & & & & & & & & & & & \\
\hline 6 & 3.935505000 & 0.974004000 & -0.886721000 & 6 & -2.021360000 & -2.287028000 & -0.172904000 & 6 & -3.498956000 & -0.802646000 & -0.526230000 \\
\hline 6 & 2.978541000 & 1.518740000 & -0.040936000 & 6 & -1.811585000 & -1.549726000 & 0.987301000 & 6 & -3.142094000 & 0.091246000 & 0.477379000 \\
\hline 6 & 3.895294000 & -0.379069000 & -1.196638000 & 6 & -1.626800000 & -1.740458000 & -1.387232000 & 6 & -2.504188000 & -1.474306000 & -1.218891000 \\
\hline 1 & 3.015361000 & 2.573731000 & 0.198936000 & 1 & -2.124574000 & -1.955467000 & 1.942253000 & 1 & -3.914833000 & 0.621913000 & 1.019458000 \\
\hline 1 & 4.635323000 & -0.816767000 & -1.852642000 & 1 & -1.793071000 & -2.278577000 & -2.311736000 & 1 & -2.759652000 & -2.170919000 & -2.006025000 \\
\hline 6 & 1.957541000 & 0.746767000 & 0.519429000 & 6 & -1.216641000 & -0.289391000 & 0.985117000 & 6 & -1.813005000 & 0.333162000 & 0.806825000 \\
\hline 6 & 2.895383000 & -1.177048000 & -0.657154000 & 6 & -1.030171000 & -0.488125000 & -1.441694000 & 6 & -1.167260000 & -1.258215000 & -0.906207000 \\
\hline 1 & 2.844751000 & -2.232321000 & -0.882608000 & 1 & -0.768990000 & -0.046914000 & -2.393334000 & 1 & -0.403766000 & -1.787031000 & -1.456788000 \\
\hline 6 & 1.948846000 & -0.609442000 & 0.184140000 & 6 & -0.840066000 & 0.236631000 & -0.262441000 & 6 & -0.811800000 & -0.362084000 & 0.101406000 \\
\hline 17 & 0.702411000 & -1.665680000 & 0.845843000 & 17 & -1.383435000 & 2.268295000 & -0.523611000 & 17 & 1.390522000 & 2.469090000 & -1.096560000 \\
\hline 1 & 4.710516000 & 1.604941000 & -1.300452000 & 1 & -2.490711000 & -3.259171000 & -0.128021000 & 1 & -4.541200000 & -0.966926000 & -0.762404000 \\
\hline 6 & -3.621064000 & 0.710321000 & 0.019207000 & 6 & 1.930713000 & -0.204595000 & -0.016487000 & 6 & 1.583492000 & -1.059490000 & 0.013197000 \\
\hline 1 & -3.688530000 & 0.535924000 & 1.096493000 & 1 & 1.725722000 & -0.670417000 & 0.948161000 & 1 & 1.271154000 & -2.078945000 & 0.267073000 \\
\hline 1 & -3.946711000 & 1.741897000 & -0.141146000 & 1 & 1.727928000 & -0.957227000 & -0.780027000 & 1 & 1.707801000 & -1.019465000 & -1.070675000 \\
\hline 6 & -4.576003000 & -0.241014000 & -0.708876000 & 6 & 3.381241000 & 0.268227000 & -0.091891000 & 6 & 2.910836000 & -0.734470000 & 0.688925000 \\
\hline 1 & -4.247439000 & -1.271198000 & -0.540625000 & 1 & 3.553256000 & 1.034909000 & 0.668693000 & 1 & 2.776820000 & -0.764493000 & 1.775285000 \\
\hline 1 & -4.497334000 & -0.066391000 & -1.786628000 & 1 & 3.554049000 & 0.747761000 & -1.059466000 & 1 & 3.202014000 & 0.286650000 & 0.435173000 \\
\hline 6 & -6.029705000 & -0.082365000 & -0.265099000 & 6 & 4.371719000 & -0.879854000 & 0.099183000 & 6 & 4.018345000 & -1.705709000 & 0.285867000 \\
\hline 1 & -6.140487000 & -0.280677000 & 0.803054000 & 1 & 4.239544000 & -1.358634000 & 1.070932000 & 1 & 3.762371000 & -2.734111000 & 0.547753000 \\
\hline 1 & -6.390376000 & 0.931641000 & -0.450229000 & 1 & 4.242768000 & -1.645061000 & -0.668108000 & 1 & 4.200547000 & -1.670717000 & -0.789515000 \\
\hline 1 & -6.687607000 & -0.769490000 & -0.798309000 & 1 & 5.399601000 & -0.522349000 & 0.042405000 & 1 & 4.954113000 & -1.459127000 & 0.787110000 \\
\hline 7 & -2.209687000 & 0.623478000 & -0.358291000 & 7 & 0.944521000 & 0.865447000 & -0.202313000 & 7 & 0.549025000 & -0.089442000 & 0.398573000 \\
\hline 1 & -2.093084000 & 0.803210000 & -1.348951000 & 1 & 1.029609000 & 1.346447000 & -1.091323000 & 1 & 0.988284000 & 1.366591000 & -0.433587000 \\
\hline 1 & -1.846161000 & -0.307376000 & -0.187111000 & 1 & 0.960731000 & 1.574651000 & 0.522310000 & 1 & 0.661646000 & 0.186382000 & 1.365419000 \\
\hline 6 & 0.928598000 & 1.362885000 & 1.423433000 & 6 & -1.035197000 & 0.484849000 & 2.260836000 & 6 & -1.456877000 & 1.321201000 & 1.885862000 \\
\hline 1 & -0.069307000 & 1.306575000 & 0.981949000 & 1 & 0.018252000 & 0.682923000 & 2.486744000 & 1 & -0.918746000 & 0.850433000 & 2.715819000 \\
\hline 1 & 0.886123000 & 0.846074000 & 2.383580000 & 1 & -1.535969000 & 1.452231000 & 2.203006000 & 1 & -0.825022000 & 2.126600000 & 1.504661000 \\
\hline 1 & 1.164183000 & 2.409764000 & 1.608686000 & 1 & -1.442520000 & -0.067449000 & 3.106367000 & 1 & -2.354540000 & 1.772584000 & 2.303952000 \\
\hline & & & & & & $=80^{\circ} \mathrm{C}$ & & & & & \\
\hline 6 & 3.396209000 & -1.054926000 & -0.061572000 & 6 & -2.443737000 & -1.171530000 & $\begin{array}{l}-0.000856000 \\
\end{array}$ & 6 & -3.283046000 & -0.258346000 & -0.104990000 \\
\hline 6 & 3.765506000 & 0.274417000 & -0.271450000 & 6 & -1.925454000 & -0.655319000 & 1.187360000 & 6 & -2.743640000 & 0.273859000 & 1.068997000 \\
\hline 6 & 2.050292000 & -1.329179000 & 0.179093000 & 6 & -1.923971000 & -0.657212000 & -1.188190000 & 6 & -2.391321000 & -0.749685000 & -1.053016000 \\
\hline 1 & 4.804367000 & 0.519405000 & -0.454861000 & 1 & -2.315490000 & -1.005003000 & 2.136479000 & 1 & -3.405477000 & 0.670987000 & 1.828931000 \\
\hline 1 & 1.736631000 & -2.351362000 & 0.351078000 & 1 & -2.312631000 & -1.008404000 & -2.137203000 & 1 & -2.773081000 & -1.164100000 & -1.977825000 \\
\hline 6 & 2.826794000 & 1.297570000 & -0.248024000 & 6 & -0.931794000 & 0.314091000 & 1.207572000 & 6 & -1.376929000 & 0.309331000 & 1.284225000 \\
\hline 6 & 1.091335000 & -0.322403000 & 0.207376000 & 6 & -0.929696000 & 0.312808000 & -1.208549000 & 6 & -1.015019000 & -0.723025000 & -0.850282000 \\
\hline 1 & 0.050345000 & -0.551707000 & 0.397301000 & 1 & -0.587729000 & 0.721793000 & -2.149080000 & 1 & -0.360624000 & -1.107744000 & -1.618525000 \\
\hline 6 & 1.496464000 & 0.985534000 & -0.008967000 & 6 & -0.432971000 & 0.814772000 & -0.000774000 & 6 & -0.491582000 & -0.192265000 & 0.326147000 \\
\hline 17 & 0.306388000 & 2.283172000 & 0.025146000 & 17 & -0.257119000 & 2.902544000 & -0.000501000 & 17 & 1.882383000 & 2.639751000 & -0.461380000 \\
\hline 6 & -3.514759000 & -0.858464000 & -0.029092000 & 6 & 1.985628000 & -0.588179000 & 0.001931000 & 6 & 1.814948000 & -1.032289000 & -0.110229000 \\
\hline 1 & -3.204161000 & -0.743204000 & -1.070862000 & 1 & 1.581077000 & -1.094417000 & 0.879341000 & 1 & 1.420742000 & -2.053342000 & -0.050830000 \\
\hline 1 & -3.634523000 & -1.933017000 & 0.134694000 & 1 & 1.578338000 & -1.095982000 & -0.873328000 & 1 & 1.874114000 & -0.767860000 & -1.167646000 \\
\hline 6 & -4.862273000 & -0.160740000 & 0.179486000 & 6 & 3.511743000 & -0.639306000 & -0.000407000 & 6 & 3.207803000 & -0.968684000 & 0.506083000 \\
\hline 1 & -4.734779000 & 0.913513000 & 0.014367000 & 1 & 3.893721000 & -0.105889000 & 0.874452000 & 1 & 3.140934000 & -1.222133000 & 1.569245000 \\
\hline 1 & -5.163777000 & -0.276728000 & 1.225387000 & 1 & 3.890869000 & -0.107324000 & -0.877386000 & 1 & 3.577282000 & 0.056976000 & 0.448602000 \\
\hline 6 & -5.960556000 & -0.698229000 & -0.737053000 & 6 & 4.038025000 & -2.074394000 & -0.000110000 & 6 & 4.189291000 & -1.914907000 & -0.182043000 \\
\hline 1 & -5.696779000 & -0.566502000 & -1.788477000 & 1 & 3.698662000 & -2.619746000 & 0.882132000 & 1 & 3.854030000 & -2.951961000 & -0.120153000 \\
\hline 1 & -6.127002000 & -1.764679000 & -0.570931000 & 1 & 3.695750000 & -2.621163000 & -0.880344000 & 1 & 4.303822000 & -1.663662000 & -1.237801000 \\
\hline 1 & -6.908167000 & -0.185799000 & -0.567741000 & 1 & 5.127661000 & -2.090739000 & -0.001890000 & 1 & 5.174834000 & -1.857080000 & 0.279571000 \\
\hline 7 & -2.412175000 & -0.397042000 & 0.816873000 & 7 & 1.434723000 & 0.772531000 & 0.001378000 & 7 & 0.905287000 & -0.086886000 & 0.554044000 \\
\hline 1 & -2.648130000 & -0.498782000 & 1.797489000 & 1 & 1.693274000 & 1.317686000 & -0.814969000 & 1 & 1.416728000 & 1.463256000 & 0.009229000 \\
\hline
\end{tabular}




\begin{tabular}{|c|c|c|c|c|c|c|c|c|c|c|c|}
\hline 1 & -2.229409000 & 0.588454000 & 0.662896000 & 1 & 1.692386000 & 1.318233000 & 0.817638000 & 1 & 1.098966000 & -0.042595000 & 1.546666000 \\
\hline 1 & 3.123327000 & 2.323812000 & -0.409477000 & 1 & -0.590636000 & 0.724395000 & 2.147839000 & 1 & -0.987042000 & 0.738741000 & 2.199437000 \\
\hline 6 & 4.418628000 & -2.159793000 & -0.118141000 & 6 & -3.506175000 & -2.240447000 & 0.000720000 & 6 & -4.772843000 & -0.309554000 & -0.321319000 \\
\hline 1 & 4.604334000 & -2.471351000 & -1.149338000 & 1 & -3.077781000 & -3.243141000 & 0.092984000 & 1 & -5.237410000 & -1.086441000 & 0.291338000 \\
\hline 1 & 5.373736000 & -1.840803000 & 0.300124000 & 1 & -4.201325000 & -2.110880000 & 0.831652000 & 1 & -5.246478000 & 0.636539000 & -0.054718000 \\
\hline 1 & 4.084783000 & -3.038606000 & 0.432827000 & 1 & -4.087770000 & -2.222083000 & -0.921631000 & 1 & -5.014824000 & -0.523758000 & -1.361799000 \\
\hline
\end{tabular}

Table S15. Coordinates of the optimized reactants, products and transition states for the 4$\mathrm{MePhBr}+\mathrm{NH}_{2}{ }^{\mathrm{n} P r}$ amination at different temperatures. The default used for thermochemistry analysis is $25^{\circ} \mathrm{C}$.

\begin{tabular}{|c|c|c|c|c|c|c|c|c|c|c|c|}
\hline \multicolumn{4}{|c|}{ Reactant } & \multicolumn{4}{|c|}{ Transition State } & \multicolumn{4}{|c|}{ Product } \\
\hline \multicolumn{12}{|c|}{$T=-20^{\circ} \mathrm{C}$} \\
\hline 6 & -2.996356000 & 1.904550000 & 0.009614000 & 6 & -2.156123000 & -1.989152000 & -0.000006000 & 6 & 3.653585000 & -0.038327000 & 0.171622000 \\
\hline 6 & -1.602492000 & 1.842693000 & 0.009155000 & 6 & -1.714740000 & -1.408421000 & 1.188908000 & 6 & 2.668127000 & -0.797085000 & 0.806767000 \\
\hline 6 & -3.704215000 & 0.703075000 & 0.010196000 & 6 & -1.714719000 & -1.408431000 & -1.188913000 & 6 & 3.247546000 & 0.948285000 & -0.726528000 \\
\hline 1 & -1.026917000 & 2.760269000 & 0.015446000 & 1 & -2.040395000 & -1.819089000 & 2.137722000 & 1 & 2.958947000 & -1.574282000 & 1.501786000 \\
\hline 1 & -4.787041000 & 0.718986000 & 0.017190000 & 1 & -2.040356000 & -1.819106000 & -2.137730000 & 1 & 3.990528000 & 1.549214000 & -1.234516000 \\
\hline 6 & -0.923377000 & 0.629462000 & 0.003655000 & 6 & -0.875412000 & -0.300979000 & 1.208449000 & 6 & 1.318696000 & -0.588829000 & 0.559557000 \\
\hline 6 & -3.049008000 & -0.522928000 & 0.004844000 & 6 & -0.875387000 & -0.300989000 & -1.208448000 & 6 & 1.900861000 & 1.173817000 & -0.982116000 \\
\hline 1 & -3.611360000 & -1.445188000 & 0.007676000 & 1 & -0.584098000 & 0.143180000 & -2.149645000 & 1 & 1.603969000 & 1.945260000 & -1.682396000 \\
\hline 6 & -1.662834000 & -0.543866000 & 0.000282000 & 6 & -0.467066000 & 0.263334000 & 0.000001000 & 6 & 0.945834000 & 0.403518000 & -0.336334000 \\
\hline 35 & -0.753979000 & -2.234887000 & -0.003397000 & 35 & -0.571239000 & 2.535030000 & -0.000003000 & 35 & -2.138056000 & -1.865913000 & -0.111251000 \\
\hline 6 & 3.814095000 & 0.991796000 & 0.002198000 & 6 & 2.180892000 & -0.796015000 & 0.000046000 & 6 & -1.134391000 & 1.620094000 & 0.337145000 \\
\hline 1 & 3.766560000 & 1.648613000 & -0.870614000 & 1 & 1.848172000 & -1.354354000 & 0.876552000 & 1 & -1.180746000 & 1.128888000 & 1.307885000 \\
\hline 1 & 3.769681000 & 1.646554000 & 0.876718000 & 1 & 1.848088000 & -1.354449000 & -0.876368000 & 1 & -0.489529000 & 2.495556000 & 0.417677000 \\
\hline 6 & 5.148351000 & 0.239545000 & -0.001078000 & 6 & 3.701813000 & -0.647278000 & -0.000035000 & 6 & -2.526810000 & 1.982522000 & -0.157115000 \\
\hline 1 & 5.184638000 & -0.414917000 & -0.877569000 & 1 & 4.008205000 & -0.068498000 & 0.875762000 & 1 & -3.103518000 & 1.064402000 & -0.278060000 \\
\hline 1 & 5.187629000 & -0.417233000 & 0.873550000 & 1 & 4.008119000 & -0.068599000 & -0.875929000 & 1 & -2.450161000 & 2.452208000 & -1.143574000 \\
\hline 6 & 6.358427000 & 1.172739000 & -0.001898000 & 6 & 4.413675000 & -1.999555000 & 0.000007000 & 6 & -3.236318000 & 2.929709000 & 0.808894000 \\
\hline 1 & 6.357554000 & 1.819574000 & -0.881735000 & 1 & 4.148703000 & -2.586165000 & 0.881347000 & 1 & -3.363683000 & 2.465974000 & 1.787925000 \\
\hline 1 & 6.360669000 & 1.817045000 & 0.879788000 & 1 & 4.148612000 & -2.586268000 & -0.881238000 & 1 & -2.679674000 & 3.859076000 & 0.945904000 \\
\hline 1 & 7.294336000 & 0.613117000 & -0.004363000 & 1 & 5.495908000 & -1.871186000 & -0.000057000 & 1 & -4.226431000 & 3.187515000 & 0.434902000 \\
\hline 7 & 2.606491000 & 0.163613000 & 0.003392000 & 7 & 1.454245000 & 0.476942000 & 0.000010000 & 7 & -0.473748000 & 0.636941000 & -0.588509000 \\
\hline 1 & 2.588678000 & -0.444759000 & 0.814146000 & 1 & 1.631023000 & 1.052272000 & -0.816999000 & 1 & -0.604743000 & 0.944547000 & -1.549908000 \\
\hline 1 & 2.585924000 & -0.443130000 & -0.808514000 & 1 & 1.631015000 & 1.052315000 & 0.816988000 & 1 & -1.053659000 & -0.326285000 & -0.485160000 \\
\hline 1 & 0.159191000 & 0.605587000 & 0.005166000 & 1 & -0.584137000 & 0.143197000 & 2.149647000 & 1 & 0.560891000 & -1.202570000 & 1.027774000 \\
\hline 6 & -3.713239000 & 3.229095000 & -0.015120000 & 6 & -3.100604000 & -3.163570000 & -0.000007000 & 6 & 5.113104000 & -0.301679000 & 0.430498000 \\
\hline 1 & -4.713826000 & 3.148780000 & 0.409260000 & 1 & -2.956856000 & -3.790064000 & -0.881163000 & 1 & 5.732363000 & 0.536991000 & 0.115136000 \\
\hline 1 & -3.166998000 & 3.987306000 & 0.546343000 & 1 & -2.956539000 & -3.790336000 & 0.880907000 & 1 & 5.300986000 & -0.484464000 & 1.488762000 \\
\hline 1 & -3.820998000 & 3.598162000 & -1.038420000 & 1 & -4.145774000 & -2.841150000 & 0.000233000 & 1 & 5.451267000 & -1.185813000 & -0.115122000 \\
\hline \multicolumn{12}{|c|}{$T=25^{\circ} \mathrm{C}$} \\
\hline 6 & 2.946713000 & 2.362914000 & -0.341083000 & 6 & 0.960539000 & 3.064142000 & $\begin{array}{l}-0.375041000 \\
\end{array}$ & 6 & 4.060558000 & -0.152476000 & -0.068527000 \\
\hline 6 & 2.322835000 & 1.938185000 & 0.823720000 & 6 & 0.921190000 & 2.432642000 & 0.862447000 & 6 & 3.181812000 & -0.581147000 & 0.916126000 \\
\hline 6 & 2.957387000 & 1.540445000 & -1.459740000 & 6 & 0.796606000 & 2.297943000 & -1.522041000 & 6 & 3.576475000 & 0.513553000 & -1.185966000 \\
\hline 1 & 2.318860000 & 2.582812000 & 1.693399000 & 1 & 1.056156000 & 3.015786000 & 1.765729000 & 1 & 3.565841000 & -1.112119000 & 1.777315000 \\
\hline 1 & 3.441185000 & 1.857355000 & -2.373584000 & 1 & 0.840178000 & 2.757021000 & -2.501346000 & 1 & 4.246531000 & 0.845757000 & -1.966270000 \\
\hline 6 & 1.690816000 & 0.694315000 & 0.918691000 & 6 & 0.719077000 & 1.058838000 & 1.003183000 & 6 & 1.803710000 & -0.366107000 & 0.833058000 \\
\hline 6 & 2.341097000 & 0.297300000 & -1.402597000 & 6 & 0.595594000 & 0.926456000 & -1.433893000 & 6 & 2.213631000 & 0.743766000 & -1.296147000 \\
\hline 1 & 2.340691000 & -0.356834000 & -2.262057000 & 1 & 0.513818000 & 0.327167000 & -2.329315000 & 1 & 1.821542000 & 1.257016000 & -2.166133000 \\
\hline 6 & 1.721866000 & -0.104095000 & -0.227097000 & 6 & 0.576197000 & 0.321591000 & -0.179065000 & 6 & 1.344235000 & 0.311379000 & -0.301130000 \\
\hline 35 & 0.890628000 & -1.839394000 & -0.217529000 & 35 & 1.772325000 & -1.643341000 & -0.204577000 & 35 & -2.059256000 & -1.721843000 & -0.327003000 \\
\hline 1 & 3.424153000 & 3.332971000 & -0.372897000 & 1 & 1.123890000 & 4.130299000 & -0.440315000 & 1 & 5.119480000 & -0.346667000 & 0.033452000 \\
\hline 6 & -3.429851000 & 0.718038000 & 0.486771000 & 6 & -2.264761000 & -0.059368000 & -0.029334000 & 6 & -0.666978000 & 1.689235000 & 0.338729000 \\
\hline 1 & -3.934804000 & 1.274631000 & 1.281330000 & 1 & -2.249526000 & 0.552434000 & 0.873775000 & 1 & -0.780780000 & 1.296120000 & 1.346087000 \\
\hline 1 & -2.841506000 & 1.452341000 & -0.069962000 & 1 & -2.244788000 & 0.628895000 & -0.876033000 & 1 & 0.053371000 & 2.507350000 & 0.363493000 \\
\hline 6 & -4.478649000 & 0.098717000 & -0.442394000 & 6 & -3.523635000 & -0.925462000 & -0.070555000 & 6 & -2.010158000 & 2.137743000 & -0.220297000 \\
\hline 1 & -5.057752000 & -0.640879000 & 0.119748000 & 1 & -3.516565000 & -1.615760000 & 0.777389000 & 1 & -2.667569000 & 1.270374000 & -0.294204000 \\
\hline 1 & -3.967653000 & -0.452334000 & -1.237886000 & 1 & -3.505576000 & -1.543103000 & -0.972806000 & 1 & -1.867652000 & 2.522232000 & -1.235714000 \\
\hline 6 & -5.420285000 & 1.135231000 & -1.054090000 & 6 & -4.800839000 & -0.086459000 & -0.044212000 & 6 & -2.647651000 & 3.217803000 & 0.651859000 \\
\hline 1 & -5.961968000 & 1.682424000 & -0.279549000 & 1 & -4.858337000 & 0.518127000 & 0.862560000 & 1 & -2.844194000 & 2.844424000 & 1.657918000 \\
\hline 1 & -4.869580000 & 1.866664000 & -1.649155000 & 1 & -4.845872000 & 0.591188000 & -0.898440000 & 1 & -2.007131000 & 4.097892000 & 0.738463000 \\
\hline 1 & -6.158969000 & 0.668077000 & -1.706357000 & 1 & -5.686186000 & -0.721121000 & -0.076586000 & 1 & -3.598592000 & 3.539313000 & 0.228862000 \\
\hline 7 & -2.495284000 & -0.214195000 & 1.118811000 & 7 & -1.011482000 & -0.817330000 & -0.065484000 & 7 & -0.080330000 & 0.583626000 & -0.495703000 \\
\hline 1 & -1.970257000 & -0.728858000 & 0.420415000 & 1 & -0.905459000 & -1.394135000 & -0.892802000 & 1 & -0.221511000 & 0.821610000 & -1.475568000 \\
\hline 1 & -2.995355000 & -0.901940000 & 1.670567000 & 1 & -0.862577000 & -1.416057000 & 0.739044000 & 1 & -0.736688000 & -0.331048000 & -0.375373000 \\
\hline 6 & 1.016098000 & 0.275886000 & 2.193847000 & 6 & 0.702807000 & 0.415288000 & 2.360574000 & 6 & 0.899746000 & -0.858541000 & 1.932293000 \\
\hline 1 & -0.063517000 & 0.178559000 & 2.055645000 & 1 & -0.260369000 & -0.052093000 & 2.589262000 & 1 & 0.660272000 & -0.056779000 & 2.635828000 \\
\hline 1 & 1.387591000 & -0.689979000 & 2.539606000 & 1 & 1.460320000 & -0.367065000 & 2.427533000 & 1 & -0.036599000 & -1.264217000 & 1.547952000 \\
\hline 1 & 1.195703000 & 1.011513000 & 2.976424000 & 1 & 0.893269000 & 1.152981000 & 3.138548000 & 1 & 1.397668000 & -1.643392000 & 2.498945000 \\
\hline \multicolumn{12}{|c|}{$T=80^{\circ} \mathrm{C}$} \\
\hline 6 & -3.228274000 & 1.644700000 & -0.033985000 & 6 & -2.156123000 & -1.989152000 & & 6 & 3.653576000 & -0.038352000 & 0.171643000 \\
\hline 6 & -1.853634000 & 1.775649000 & 0.163815000 & 6 & -1.714740000 & -1.408421000 & 1.188908000 & 6 & 2.668126000 & -0.797161000 & 0.806730000 \\
\hline 6 & -3.742766000 & 0.361651000 & -0.219856000 & 6 & -1.714719000 & -1.408431000 & -1.188913000 & 6 & 3.247534000 & 0.948297000 & -0.726470000 \\
\hline 1 & -1.427166000 & 2.759332000 & 0.317711000 & 1 & -2.040395000 & -1.819089000 & 2.137722000 & 1 & 2.958943000 & -1.574388000 & 1.501718000 \\
\hline 1 & -4.807019000 & 0.227097000 & -0.368747000 & 1 & -2.040356000 & -1.819106000 & -2.137730000 & 1 & 3.990522000 & 1.549251000 & -1.234418000 \\
\hline 6 & -1.006367000 & 0.672856000 & 0.172610000 & 6 & -0.875412000 & -0.300979000 & 1.208449000 & 6 & 1.318690000 & -0.588903000 & 0.559514000 \\
\hline 6 & -2.917532000 & -0.756612000 & -0.215779000 & 6 & -0.875387000 & -0.300989000 & -1.208448000 & 6 & 1.900857000 & 1.173809000 & -0.982085000 \\
\hline 1 & -3.331269000 & -1.744106000 & -0.359269000 & 1 & -0.584098000 & 0.143180000 & -2.149645000 & 1 & 1.603966000 & 1.945261000 & -1.682355000 \\
\hline 6 & -1.555557000 & -0.585671000 & -0.019966000 & 6 & -0.467066000 & 0.263334000 & 0.000001000 & 6 & 0.945830000 & 0.403473000 & -0.336342000 \\
\hline 35 & -0.414719000 & -2.130493000 & -0.010120000 & 35 & -0.571239000 & 2.535030000 & -0.000003000 & 35 & -2.138092000 & -1.865912000 & -0.111240000 \\
\hline 6 & 3.643023000 & 1.139462000 & -0.023785000 & 6 & 2.180892000 & -0.796015000 & 0.000046000 & 6 & -1.134372000 & 1.620070000 & 0.337170000 \\
\hline 1 & 3.387406000 & 1.147971000 & -1.086636000 & 1 & 1.848172000 & -1.354354000 & 0.876552000 & 1 & $\begin{array}{r}-1.180787000 \\
\end{array}$ & 1.128827000 & 1.307888000 \\
\hline 1 & 3.730933000 & 2.187571000 & 0.275338000 & 1 & 1.848088000 & -1.354449000 & -0.876368000 & 1 & -0.489465000 & 2.495493000 & 0.417757000 \\
\hline 6 & 4.993427000 & 0.442631000 & 0.168500000 & 6 & 3.701813000 & -0.647278000 & -0.000035000 & 6 & -2.526752000 & 1.982596000 & -0.157130000 \\
\hline
\end{tabular}




\begin{tabular}{|c|c|c|c|c|c|c|c|c|c|c|c|}
\hline 1 & 4.898553000 & -0.604844000 & -0.133685000 & 1 & 4.008205000 & -0.068498000 & 0.875762000 & 1 & -3.103515000 & 1.064515000 & -0.278114000 \\
\hline 1 & 5.239154000 & 0.433163000 & 1.235101000 & 1 & 4.008119000 & -0.068599000 & -0.875929000 & 1 & -2.450036000 & 2.452296000 & -1.143577000 \\
\hline 6 & 6.124231000 & 1.106751000 & -0.616032000 & 6 & 4.413675000 & -1.999555000 & 0.000007000 & 6 & -3.236234000 & 2.929813000 & 0.808869000 \\
\hline 1 & 5.917297000 & 1.100778000 & -1.688216000 & 1 & 4.148703000 & -2.586165000 & 0.881347000 & 1 & -3.363687000 & 2.466061000 & 1.787881000 \\
\hline 1 & 6.257535000 & 2.147305000 & -0.312627000 & 1 & 4.148612000 & -2.586268000 & -0.881238000 & 1 & -2.679520000 & 3.859129000 & 0.945933000 \\
\hline 1 & 7.073486000 & 0.593125000 & -0.460176000 & 1 & 5.495908000 & -1.871186000 & -0.000057000 & 1 & -4.226306000 & 3.187711000 & 0.434833000 \\
\hline 7 & 2.509990000 & 0.561244000 & 0.701714000 & 7 & 1.454245000 & 0.476942000 & 0.000010000 & 7 & -0.473750000 & 0.636918000 & -0.588505000 \\
\hline 1 & 2.692504000 & 0.550686000 & 1.698865000 & 1 & 1.631023000 & 1.052272000 & -0.816999000 & 1 & -0.604742000 & 0.944551000 & -1.549896000 \\
\hline 1 & 2.358992000 & -0.402809000 & 0.425743000 & 1 & 1.631015000 & 1.052315000 & 0.816988000 & 1 & -1.053673000 & -0.326292000 & -0.485175000 \\
\hline 1 & 0.057551000 & 0.798001000 & 0.330305000 & 1 & -0.584137000 & 0.143197000 & 2.149647000 & 1 & 0.560884000 & -1.202671000 & 1.027691000 \\
\hline 6 & -4.126326000 & 2.853438000 & -0.070831000 & 6 & -3.100604000 & -3.163570000 & -0.000007000 & 6 & 5.113115000 & -0.301658000 & 0.430472000 \\
\hline 1 & -5.136630000 & 2.609114000 & 0.257193000 & 1 & -2.956856000 & -3.790064000 & -0.881163000 & 1 & 5.732170000 & 0.537712000 & 0.116549000 \\
\hline 1 & -3.746221000 & 3.650937000 & 0.567565000 & 1 & -2.956539000 & -3.790336000 & 0.880907000 & 1 & 5.300812000 & -0.486039000 & 1.488487000 \\
\hline 1 & -4.201213000 & 3.256076000 & -1.084387000 & 1 & -4.145774000 & -2.841150000 & 0.000233000 & 1 & 5.451700000 & -1.184805000 & -0.116491000 \\
\hline
\end{tabular}

Table S16. Coordinates of the optimized reactants, products and transition states for the $\mathrm{PhBr}+$ $\mathrm{NH}_{2}{ }^{\mathrm{n}} \mathrm{Pr}$ amination at $80{ }^{\circ} \mathrm{C}$ in different solvent environments.

\begin{tabular}{|c|c|c|c|c|c|c|c|c|c|c|c|}
\hline \multicolumn{4}{|c|}{ Reactant } & \multicolumn{4}{|c|}{ Transition State } & \multicolumn{4}{|c|}{ Product } \\
\hline \multicolumn{12}{|c|}{ Gas Phase } \\
\hline 6 & -3.110868000 & 2.449228000 & -0.017272000 & 6 & -0.903514000 & 3.104103000 & -0.000333000 & 6 & 3.995812000 & 0.265290000 & 0.361132000 \\
\hline 6 & -1.729192000 & 2.320168000 & 0.060898000 & 6 & -0.829958000 & 2.397196000 & -1.196097000 & 6 & 3.700682000 & -0.725695000 & -0.568120000 \\
\hline 6 & -3.907480000 & 1.312567000 & -0.092268000 & 6 & -0.830860000 & 2.397454000 & 1.195646000 & 6 & 2.968444000 & 0.970913000 & 0.976955000 \\
\hline 1 & -1.103069000 & 3.200612000 & 0.119259000 & 1 & -0.903927000 & 2.919398000 & -2.141778000 & 1 & 4.496817000 & -1.271466000 & -1.055227000 \\
\hline 1 & -4.983763000 & 1.403716000 & -0.153226000 & 1 & -0.905522000 & 2.919865000 & 2.141157000 & 1 & 3.196993000 & 1.751502000 & 1.689231000 \\
\hline 6 & -1.131122000 & 1.063348000 & 0.065105000 & 6 & -0.678824000 & 1.016180000 & -1.212818000 & 6 & 2.377420000 & -1.016190000 & -0.875961000 \\
\hline 6 & -3.329156000 & 0.047848000 & -0.089410000 & 6 & -0.679741000 & 1.016448000 & 1.212787000 & 6 & 1.640955000 & 0.692108000 & 0.673907000 \\
\hline 1 & -0.054829000 & 0.962237000 & 0.125261000 & 1 & -0.664341000 & 0.479771000 & -2.150880000 & 1 & 2.141820000 & -1.786850000 & -1.599763000 \\
\hline 1 & -3.942114000 & -0.839544000 & -0.147542000 & 1 & -0.665913000 & 0.480241000 & 2.150973000 & 1 & 0.830249000 & 1.255558000 & 1.115789000 \\
\hline 6 & -1.947591000 & -0.055837000 & -0.010545000 & 6 & -0.613909000 & 0.324068000 & 0.000085000 & 6 & 1.362235000 & -0.305231000 & -0.250164000 \\
\hline 35 & -1.149887000 & -1.802637000 & -0.006775000 & 35 & -1.861471000 & -1.563843000 & -0.000142000 & 35 & -1.839562000 & 1.796894000 & -0.133170000 \\
\hline 1 & -3.565410000 & 3.430641000 & -0.019785000 & 1 & -1.027686000 & 4.177233000 & -0.000495000 & 1 & 5.026063000 & 0.491843000 & 0.599095000 \\
\hline 6 & 3.632192000 & 0.824552000 & 0.001668000 & 6 & 2.191795000 & -0.083250000 & 0.001510000 & 6 & -0.674650000 & -1.635767000 & 0.329619000 \\
\hline 1 & 3.592834000 & 1.305144000 & -0.979597000 & 1 & 2.181114000 & 0.568269000 & -0.873514000 & 1 & 0.010349000 & -2.478768000 & 0.424395000 \\
\hline 1 & 3.778738000 & 1.629029000 & 0.727478000 & 1 & 2.181973000 & 0.564833000 & 0.879070000 & 1 & -0.781307000 & -1.160295000 & 1.303395000 \\
\hline 6 & 4.829801000 & -0.129032000 & 0.052318000 & 6 & 3.434960000 & -0.971709000 & -0.000861000 & 6 & -2.028005000 & -2.061894000 & -0.219658000 \\
\hline 1 & 4.676500000 & -0.930289000 & -0.677228000 & 1 & 3.411769000 & -1.624267000 & -0.878005000 & 1 & -1.891567000 & -2.515174000 & -1.207281000 \\
\hline 1 & 4.858817000 & -0.610566000 & 1.034799000 & 1 & 3.412773000 & -1.627633000 & 0.873787000 & 1 & -2.645908000 & -1.172723000 & -0.352322000 \\
\hline 6 & 6.161007000 & 0.570413000 & -0.219443000 & 6 & 4.725619000 & -0.152996000 & -0.000045000 & 6 & -2.722705000 & -3.055375000 & 0.709990000 \\
\hline 1 & 6.168797000 & 1.035220000 & -1.207546000 & 1 & 4.786587000 & 0.489492000 & -0.880043000 & 1 & -2.123315000 & -3.955597000 & 0.860408000 \\
\hline 1 & 6.351399000 & 1.355690000 & 0.515063000 & 1 & 4.787789000 & 0.485854000 & 0.882514000 & 1 & -2.911972000 & -2.609508000 & 1.687313000 \\
\hline 1 & 6.995595000 & -0.130208000 & -0.177694000 & 1 & 5.600382000 & -0.802925000 & -0.001981000 & 1 & -3.682709000 & -3.360812000 & 0.295610000 \\
\hline 7 & 2.320520000 & 0.225708000 & 0.257775000 & 7 & 0.925397000 & -0.822972000 & 0.000624000 & 7 & -0.032133000 & -0.608654000 & -0.560800000 \\
\hline 1 & 2.296880000 & -0.205003000 & 1.175280000 & 1 & 0.792234000 & -1.410264000 & 0.817592000 & 1 & -0.667875000 & 0.324908000 & -0.471026000 \\
\hline 1 & 2.126472000 & -0.513864000 & -0.408120000 & 1 & 0.793058000 & -1.409510000 & -0.817027000 & 1 & -0.109150000 & -0.912274000 & -1.529252000 \\
\hline \multicolumn{12}{|c|}{ Toluene $(\varepsilon=2.37)$} \\
\hline 6 & -2.688600000 & 2.696061000 & 0.000561000 & 6 & -0.853864000 & 3.106579000 & -0.000139000 & 6 & -3.997946000 & -0.355144000 & 0.356468000 \\
\hline 6 & -1.350950000 & 2.320243000 & -0.046597000 & 6 & -0.798893000 & 2.398550000 & -1.196651000 & 6 & -3.722531000 & 0.597464000 & -0.618303000 \\
\hline 6 & -3.678956000 & 1.721558000 & 0.052215000 & 6 & -0.798845000 & 2.398665000 & 1.196439000 & 6 & -2.957468000 & -0.992533000 & 1.022981000 \\
\hline 1 & -0.575030000 & 3.073031000 & -0.087103000 & 1 & -0.856570000 & 2.922545000 & -2.142451000 & 1 & -4.529099000 & 1.089271000 & -1.143742000 \\
\hline 1 & -4.722197000 & 2.005282000 & 0.088908000 & 1 & -0.856483000 & 2.922749000 & 2.142191000 & 1 & -3.170167000 & -1.742241000 & 1.772075000 \\
\hline 6 & -0.989981000 & 0.975711000 & -0.042588000 & 6 & -0.685824000 & 1.013170000 & -1.213133000 & 6 & -2.405696000 & 0.918495000 & -0.924156000 \\
\hline 6 & -3.338598000 & 0.372806000 & 0.057419000 & 6 & -0.685775000 & 1.013286000 & 1.213047000 & 6 & -1.636048000 & -0.681382000 & 0.724282000 \\
\hline 1 & 0.052437000 & 0.685934000 & -0.081088000 & 1 & -0.676030000 & 0.476671000 & -2.150821000 & 1 & -2.184491000 & 1.657132000 & -1.684355000 \\
\hline 1 & -4.103688000 & -0.388420000 & 0.097505000 & 1 & -0.675943000 & 0.476877000 & 2.150786000 & 1 & -0.816636000 & -1.188614000 & 1.214132000 \\
\hline 6 & -1.996609000 & 0.022323000 & 0.010024000 & 6 & -0.631877000 & 0.324711000 & -0.000011000 & 6 & -1.379805000 & 0.275172000 & -0.246728000 \\
\hline 35 & -1.523155000 & -1.839588000 & 0.016918000 & 35 & -1.892907000 & -1.539778000 & 0.000101000 & 35 & 1.932440000 & -1.815084000 & -0.127324000 \\
\hline 1 & -2.958794000 & 3.743258000 & -0.002953000 & 1 & -0.948252000 & 4.182687000 & -0.000189000 & 1 & -5.023329000 & -0.605336000 & 0.591155000 \\
\hline 6 & 3.755767000 & 0.628535000 & 0.007295000 & 6 & 2.202496000 & -0.109745000 & -0.000006000 & 6 & 0.590790000 & 1.718914000 & 0.304207000 \\
\hline 1 & 3.840692000 & 1.435122000 & -0.725694000 & 1 & 2.193263000 & 0.538610000 & -0.877305000 & 1 & -0.122342000 & 2.542288000 & 0.304760000 \\
\hline 1 & 3.709050000 & 1.110356000 & 0.987530000 & 1 & 2.193289000 & 0.538616000 & 0.877288000 & 1 & 0.652487000 & 1.312671000 & 1.311901000 \\
\hline 6 & 5.003711000 & -0.256057000 & -0.064204000 & 6 & 3.449032000 & -0.994333000 & -0.000021000 & 6 & 1.956429000 & 2.152299000 & -0.205256000 \\
\hline 1 & 5.043642000 & -0.734234000 & -1.047799000 & 1 & 3.427278000 & -1.647578000 & -0.876335000 & 1 & 1.857919000 & 2.536827000 & -1.224856000 \\
\hline 1 & 4.906795000 & -1.064147000 & 0.667337000 & 1 & 3.427295000 & -1.647582000 & 0.876292000 & 1 & 2.611283000 & 1.281347000 & -0.251063000 \\
\hline 6 & 6.297439000 & 0.517325000 & 0.187497000 & 6 & 4.736740000 & -0.171369000 & -0.000032000 & 6 & 2.567540000 & 3.226917000 & 0.693023000 \\
\hline 1 & 6.429208000 & 1.315173000 & -0.546446000 & 1 & 4.796093000 & 0.469179000 & -0.881599000 & 1 & 1.930838000 & 4.111758000 & 0.750380000 \\
\hline 1 & 6.295777000 & 0.977622000 & 1.177767000 & 1 & 4.796117000 & 0.469166000 & 0.881542000 & 1 & 2.716695000 & 2.852769000 & 1.706679000 \\
\hline 1 & 7.169515000 & -0.134693000 & 0.127355000 & 1 & 5.612967000 & -0.819452000 & -0.000049000 & 1 & 3.537888000 & 3.538584000 & 0.308789000 \\
\hline 7 & 2.475420000 & -0.049325000 & -0.219233000 & 7 & 0.939976000 & -0.854924000 & 0.000014000 & 7 & 0.011390000 & 0.620117000 & -0.552374000 \\
\hline 1 & 2.350772000 & -0.799099000 & 0.452243000 & 1 & 0.819868000 & -1.441413000 & 0.819247000 & 1 & 0.648176000 & -0.253015000 & -0.440432000 \\
\hline 1 & 2.464964000 & -0.485358000 & -1.134801000 & 1 & 0.819863000 & -1.441445000 & $\begin{array}{r}-0.819194000 \\
\end{array}$ & 1 & 0.084064000 & 0.892465000 & -1.531567000 \\
\hline \multicolumn{12}{|c|}{ Ethanol $(\varepsilon=24.85)$} \\
\hline 6 & -2.561829000 & 2.758688000 & -0.014874000 & 6 & -0.783621000 & 3.109954000 & -0.000142000 & 6 & -3.989668000 & -0.454311000 & 0.357492000 \\
\hline 6 & -3.606775000 & 1.841041000 & -0.008967000 & 6 & -0.752294000 & 2.400845000 & -1.197536000 & 6 & -3.734840000 & 0.410390000 & -0.701261000 \\
\hline 6 & -1.245983000 & 2.308682000 & -0.009439000 & 6 & -0.752284000 & 2.400963000 & 1.197322000 & 6 & -2.937781000 & -0.962909000 & 1.111805000 \\
\hline 1 & -4.633022000 & 2.182549000 & -0.013159000 & 1 & -0.786686000 & 2.926511000 & -2.143496000 & 1 & -4.549346000 & 0.803978000 & -1.292706000 \\
\hline 1 & -0.428891000 & 3.017640000 & -0.014056000 & 1 & -0.786667000 & 2.926722000 & 2.143231000 & 1 & -3.133365000 & -1.640651000 & 1.930739000 \\
\hline 6 & -3.343228000 & 0.474634000 & 0.002382000 & 6 & -0.689138000 & 1.011459000 & -1.213840000 & 6 & -2.428159000 & 0.772395000 & -1.006928000 \\
\hline 6 & -0.961925000 & 0.945437000 & 0.001953000 & 6 & -0.689127000 & 1.011578000 & 1.213762000 & 6 & -1.626680000 & -0.609699000 & 0.814449000 \\
\hline 1 & -4.151933000 & -0.241135000 & 0.006989000 & 1 & -0.684862000 & 0.475196000 & -2.151423000 & 1 & -2.223956000 & 1.444645000 & -1.829608000 \\
\hline 1 & 0.062996000 & 0.597086000 & 0.005250000 & 1 & -0.684840000 & 0.475408000 & 2.151398000 & 1 & -0.802275000 & -1.012540000 & 1.385512000 \\
\hline 6 & -2.022013000 & 0.049958000 & 0.007642000 & 6 & -0.651854000 & 0.325918000 & -0.000005000 & 6 & -1.392301000 & 0.256342000 & -0.243088000 \\
\hline 35 & -1.651501000 & -1.836489000 & 0.023175000 & 35 & -1.929804000 & -1.508119000 & 0.000090000 & 35 & 2.018428000 & -1.845432000 & -0.118999000 \\
\hline 1 & -2.772317000 & 3.819304000 & -0.023730000 & 1 & -0.837494000 & 4.188910000 & -0.000195000 & 1 & -5.007079000 & -0.734944000 & 0.591833000 \\
\hline 6 & 3.812284000 & 0.530131000 & 0.125179000 & 6 & 2.207379000 & -0.145882000 & 0.000023000 & 6 & 0.505314000 & 1.811025000 & 0.274141000 \\
\hline 1 & 3.821303000 & 1.450906000 & -0.464057000 & 1 & 2.199989000 & 0.500302000 & -0.878919000 & 1 & -0.227863000 & 2.610116000 & 0.185495000 \\
\hline
\end{tabular}




\begin{tabular}{|c|c|c|c|c|c|c|c|c|c|}
\hline 1 & 3.813299000 & 0.836284000 & 1.174644000 & 1 & 2.200042000 & 0.500272000 & 0.878986000 & 1 & 0.521428000 \\
\hline 6 & 5.081247000 & -0.273428000 & -0.169826000 & 6 & 3.457014000 & -1.027071000 & -0.000032000 & 6 & 1.882006000 \\
\hline 1 & 5.070455000 & -0.578828000 & -1.220578000 & 1 & 3.436121000 & -1.679060000 & -0.876914000 & 1 & 1.827126000 \\
\hline 1 & 5.061913000 & -1.194452000 & 0.420678000 & 1 & 3.436167000 & -1.679107000 & 0.876816000 & 1 & 2.569072000 \\
\hline 6 & 6.361788000 & 0.505348000 & 0.128869000 & 6 & 4.741442000 & -0.199116000 & -0.000043000 & 6 & 2.408742000 \\
\hline 1 & 6.418202000 & 1.415983000 & -0.471233000 & 1 & 4.797853000 & 0.441379000 & -0.882013000 & 1 & 1.745993000 \\
\hline 1 & 6.408745000 & 0.798429000 & 1.179861000 & 1 & 4.797904000 & 0.441324000 & 0.881963000 & 1 & 2.501888000 \\
\hline 1 & 7.249046000 & -0.090386000 & -0.088779000 & 1 & 5.619895000 & -0.844501000 & -0.000089000 & 1 & 3.392593000 \\
\hline 7 & 2.543457000 & -0.159639000 & -0.139561000 & 7 & 0.947854000 & -0.894330000 & 0.000046000 & 7 & -0.010437000 \\
\hline 1 & 2.493282000 & -1.012523000 & 0.407482000 & 1 & 0.837822000 & -1.480359000 & 0.820849000 & 1 & 0.639461000 \\
\hline 1 & 2.503857000 & -0.449196000 & -1.111033000 & 1 & 0.837827000 & -1.480415000 & -0.820718000 & 1 & 0.054621000 \\
\hline & & & & & & $=35.00$ & & & \\
\hline 6 & 2.563930000 & 2.758352000 & 0.015093000 & 6 & -0.779665000 & 3.110101000 & -0.000141000 & 6 & -3.988705000 \\
\hline 6 & 3.608480000 & 1.840234000 & 0.008955000 & 6 & -0.749544000 & 2.400941000 & -1.197573000 & 6 & -3.734454000 \\
\hline 6 & 1.247851000 & 2.308959000 & 0.009817000 & 6 & -0.749536000 & 2.401059000 & 1.197360000 & 6 & -2.936709000 \\
\hline 1 & 4.634874000 & 2.181292000 & 0.013042000 & 1 & -0.782758000 & 2.926663000 & -2.143542000 & 1 & -4.549034000 \\
\hline 1 & 0.431119000 & 3.018325000 & 0.014648000 & 1 & -0.782743000 & 2.926873000 & 2.143278000 & 1 & -3.131779000 \\
\hline 6 & 3.344348000 & 0.473918000 & -0.002447000 & 6 & -0.688906000 & 1.011412000 & -1.213876000 & 6 & -2.428296000 \\
\hline 6 & 0.963232000 & 0.945819000 & -0.001639000 & 6 & -0.688897000 & 1.011531000 & 1.213799000 & 6 & -1.626155000 \\
\hline 1 & 4.152773000 & -0.242163000 & -0.007207000 & 1 & -0.684992000 & 0.475170000 & -2.151467000 & 1 & -2.224672000 \\
\hline 1 & -0.061789000 & 0.597777000 & -0.004863000 & 1 & -0.684974000 & 0.475381000 & 2.151442000 & 1 & -0.801846000 \\
\hline 6 & 2.022935000 & 0.049866000 & -0.007551000 & 6 & -0.652558000 & 0.325911000 & -0.000005000 & 6 & -1.392363000 \\
\hline 35 & 1.651453000 & -1.836446000 & -0.023118000 & 35 & -1.931677000 & -1.506328000 & 0.000089000 & 35 & 2.021143000 \\
\hline 1 & 2.774891000 & 3.818866000 & 0.024018000 & 1 & -0.831494000 & 4.189149000 & -0.000194000 & 1 & -5.005710000 \\
\hline 6 & -3.814010000 & 0.529562000 & -0.127510000 & 6 & 2.207242000 & -0.147878000 & 0.000023000 & 6 & 0.501989000 \\
\hline 1 & -3.821810000 & 1.452234000 & 0.458753000 & 1 & 2.199968000 & 0.498197000 & -0.879003000 & 1 & -0.232100000 \\
\hline 1 & -3.816041000 & 0.832184000 & -1.178000000 & 1 & 2.200021000 & 0.498167000 & 0.879072000 & 1 & 0.516691000 \\
\hline 6 & -5.083064000 & -0.272308000 & 0.171579000 & 6 & 3.456956000 & -1.028985000 & -0.000031000 & 6 & 1.878805000 \\
\hline 1 & -5.071221000 & -0.574202000 & 1.223324000 & 1 & 3.436083000 & -1.680918000 & -0.876939000 & 1 & 1.825136000 \\
\hline 1 & -5.064871000 & -1.195247000 & -0.415945000 & 1 & 3.436130000 & -1.680965000 & 0.876842000 & 1 & 2.567208000 \\
\hline 6 & -6.363493000 & 0.506220000 & -0.128246000 & 6 & 4.741269000 & -0.200843000 & -0.000044000 & 6 & 2.402412000 \\
\hline 1 & -6.418658000 & 1.418888000 & 0.468877000 & 1 & 4.797567000 & 0.439649000 & -0.882033000 & 1 & 1.738517000 \\
\hline 1 & -6.411489000 & 0.795780000 & -1.180171000 & 1 & 4.797620000 & 0.439594000 & 0.881982000 & 1 & 2.493956000 \\
\hline 1 & -7.250830000 & -0.088284000 & 0.092445000 & 1 & 5.619814000 & -0.846119000 & -0.000090000 & 1 & 3.386447000 \\
\hline 7 & -2.545298000 & -0.160263000 & 0.138135000 & 7 & 0.947755000 & -0.896311000 & 0.000048000 & 7 & -0.010863000 \\
\hline 1 & -2.496995000 & -1.015328000 & -0.405696000 & 1 & 0.837993000 & -1.482311000 & 0.820905000 & 1 & 0.639598000 \\
\hline 1 & -2.504984000 & -0.446400000 & 1.110649000 & 1 & 0.837998000 & -1.482369000 & -0.820768000 & 1 & 0.054483000 \\
\hline & & & & & $D M F$ & 27 & 2) & & \\
\hline 6 & -2.679414000 & 2.713778000 & -0.000184000 & 6 & -0.779069000 & 3.110123000 & -0.000141000 & 6 & -3.980037000 \\
\hline 6 & -1.345686000 & 2.319833000 & -0.000219000 & 6 & -0.749128000 & 2.400955000 & -1.197579000 & 6 & -3.718735000 \\
\hline 6 & -3.684761000 & 1.752858000 & -0.000039000 & 6 & -0.749120000 & 2.401072000 & 1.197366000 & 6 & -2.933016000 \\
\hline 1 & -0.559266000 & 3.062633000 & -0.000331000 & 1 & -0.782166000 & 2.926685000 & -2.143549000 & 1 & -4.529318000 \\
\hline 1 & -4.724481000 & 2.050874000 & -0.000010000 & 1 & -0.782151000 & 2.926895000 & 2.143285000 & 1 & -3.133493000 \\
\hline 6 & -1.004637000 & 0.969718000 & -0.000111000 & 6 & -0.688867000 & 1.011405000 & -1.213881000 & 6 & -2.410548000 \\
\hline 6 & -3.364024000 & 0.398713000 & 0.000070000 & 6 & -0.688859000 & 1.011524000 & 1.213804000 & 6 & -1.620551000 \\
\hline 1 & 0.033876000 & 0.664505000 & -0.000137000 & 1 & -0.685009000 & 0.475167000 & -2.151473000 & 1 & -2.201445000 \\
\hline 1 & -4.141891000 & -0.350467000 & 0.000182000 & 1 & -0.684991000 & 0.475378000 & 2.151448000 & 1 & -0.800325000 \\
\hline 6 & -2.026022000 & 0.030223000 & 0.000032000 & 6 & -0.652660000 & 0.325909000 & -0.000005000 & 6 & -1.379638000 \\
\hline 35 & -1.576656000 & -1.839145000 & 0.000184000 & 35 & -1.931958000 & -1.506057000 & 0.000089000 & 35 & 1.991904000 \\
\hline 1 & -2.934587000 & 3.764565000 & -0.000269000 & 1 & -0.830591000 & 4.189184000 & -0.000194000 & 1 & -4.998547000 \\
\hline 6 & 3.825754000 & 0.620185000 & -0.000121000 & 6 & 2.207219000 & -0.148179000 & 0.000024000 & 6 & 0.517821000 \\
\hline 1 & 3.853301000 & 1.274745000 & -0.875256000 & 1 & 2.199962000 & 0.497880000 & -0.879015000 & 1 & -0.213189000 \\
\hline 1 & 3.853283000 & 1.274827000 & 0.874952000 & 1 & 2.200015000 & 0.497850000 & 0.879084000 & 1 & 0.528480000 \\
\hline 6 & 5.064929000 & -0.278437000 & -0.000065000 & 6 & 3.456944000 & -1.029274000 & -0.000031000 & 6 & 1.897539000 \\
\hline 1 & 5.027491000 & -0.932467000 & -0.876516000 & 1 & 3.436074000 & -1.681199000 & -0.876942000 & 1 & 1.847850000 \\
\hline 1 & 5.027473000 & -0.932383000 & 0.876447000 & 1 & 3.436121000 & -1.681246000 & 0.876846000 & 1 & 2.583517000 \\
\hline 6 & 6.372849000 & 0.512062000 & -0.000089000 & 6 & 4.741240000 & -0.201104000 & -0.000044000 & 6 & 2.422516000 \\
\hline 1 & 6.446073000 & 1.152669000 & -0.881518000 & 1 & 4.797521000 & 0.439387000 & -0.882037000 & 1 & 1.761386000 \\
\hline 1 & 6.446052000 & 1.152757000 & 0.881277000 & 1 & 4.797574000 & 0.439332000 & 0.881985000 & 1 & 2.510350000 \\
\hline 1 & 7.238309000 & -0.151614000 & -0.000046000 & 1 & 5.619798000 & -0.846364000 & -0.000090000 & 1 & 3.408419000 \\
\hline 7 & 2.532599000 & -0.075438000 & -0.000102000 & 7 & 0.947737000 & -0.896609000 & 0.000048000 & 7 & 0.003431000 \\
\hline 1 & 2.467602000 & -0.683000000 & 0.809749000 & 1 & 0.838013000 & -1.482604000 & 0.820913000 & 1 & 0.652693000 \\
\hline 1 & 2.467622000 & -0.683081000 & -0.809894000 & 1 & 0.838018000 & -1.482662000 & -0.820776000 & 1 & 0.070861000 \\
\hline & & & & & & $=50.00$ & & & \\
\hline 6 & $\begin{array}{l}-2.676924000 \\
\end{array}$ & 2.715136000 & -0.000032000 & 6 & -0.776611000 & 3.110210000 & $\begin{array}{l}-0.000141000 \\
\end{array}$ & 6 & $\begin{array}{l}-3.987916000 \\
\end{array}$ \\
\hline 6 & -3.683440000 & 1.755425000 & -0.000565000 & 6 & -0.747412000 & 2.401011000 & -1.197601000 & 6 & -3.733858000 \\
\hline 6 & -1.343654000 & 2.319592000 & 0.000564000 & 6 & -0.747405000 & 2.401128000 & 1.197389000 & 6 & -2.936019000 \\
\hline 1 & -4.722797000 & 2.054690000 & -0.001026000 & 1 & -0.779727000 & 2.926774000 & -2.143577000 & 1 & -4.548347000 \\
\hline 1 & -0.556366000 & 3.061472000 & 0.000992000 & 1 & -0.779715000 & 2.926983000 & 2.143314000 & 1 & -3.130890000 \\
\hline 6 & -3.364349000 & 0.400874000 & -0.000508000 & 6 & -0.688699000 & 1.011377000 & -1.213903000 & 6 & -2.428035000 \\
\hline 6 & -1.004264000 & 0.969051000 & 0.000622000 & 6 & -0.688692000 & 1.011496000 & 1.213826000 & 6 & -1.625815000 \\
\hline 1 & -4.143149000 & -0.347333000 & -0.000921000 & 1 & -0.685070000 & 0.475152000 & -2.151501000 & 1 & -2.224640000 \\
\hline 1 & 0.033844000 & 0.662491000 & 0.001075000 & 1 & -0.685055000 & 0.475362000 & 2.151476000 & 1 & -0.801744000 \\
\hline 6 & -2.026794000 & 0.030786000 & 0.000080000 & 6 & -0.653072000 & 0.325900000 & -0.000005000 & 6 & -1.392222000 \\
\hline 35 & -1.579583000 & -1.839120000 & 0.000140000 & 35 & -1.933115000 & -1.504941000 & 0.000088000 & 35 & 2.022772000 \\
\hline 1 & -2.930823000 & 3.766227000 & -0.000075000 & 1 & -0.826878000 & 4.189326000 & -0.000193000 & 1 & -5.004660000 \\
\hline 6 & 3.827459000 & 0.618722000 & 0.000081000 & 6 & 2.207114000 & -0.149418000 & 0.000024000 & 6 & 0.499772000 \\
\hline 1 & 3.854787000 & 1.273895000 & -0.874598000 & 1 & 2.199929000 & 0.496573000 & -0.879067000 & 1 & -0.234962000 \\
\hline 1 & 3.854617000 & 1.272715000 & 0.875651000 & 1 & 2.199983000 & 0.496544000 & 0.879136000 & 1 & 0.513413000 \\
\hline 6 & 5.067016000 & -0.279350000 & -0.000398000 & 6 & 3.456884000 & -1.030468000 & -0.000031000 & 6 & 1.876707000 \\
\hline 1 & 5.029917000 & -0.932775000 & -0.877308000 & 1 & 3.436026000 & -1.682359000 & -0.876956000 & 1 & 1.824000000 \\
\hline 1 & 5.029788000 & -0.933882000 & 0.875681000 & 1 & 3.436073000 & -1.682405000 & 0.876862000 & 1 & 2.566106000 \\
\hline 6 & 6.374567000 & 0.511765000 & 0.000202000 & 6 & 4.741111000 & -0.202185000 & -0.000044000 & 6 & 2.397964000 \\
\hline 1 & 6.447544000 & 1.152979000 & -0.880807000 & 1 & 4.797326000 & 0.438303000 & -0.882049000 & 1 & 1.733259000 \\
\hline 1 & 6.447388000 & 1.151901000 & 0.882007000 & 1 & 4.797379000 & 0.438250000 & 0.881996000 & 1 & 2.488199000 \\
\hline 1 & 7.240327000 & -0.151522000 & -0.000127000 & 1 & 5.619725000 & -0.847379000 & -0.000091000 & 1 & 3.382181000 \\
\hline 7 & 2.534614000 & -0.077584000 & -0.000527000 & 7 & 0.947649000 & -0.897830000 & 0.000049000 & 7 & -0.010980000 \\
\hline 1 & 2.470142000 & -0.685930000 & 0.808787000 & 1 & 0.838079000 & -1.483807000 & 0.820946000 & 1 & 0.639882000 \\
\hline 1 & 2.470190000 & -0.684643000 & -0.810811000 & 1 & 0.838084000 & -1.483866000 & -0.820806000 & 1 & 0.054561000 \\
\hline
\end{tabular}




\begin{tabular}{|c|c|c|c|c|c|c|c|c|c|c|c|}
\hline \multicolumn{12}{|c|}{$\varepsilon=65.00$} \\
\hline 6 & -2.657051000 & 2.720038000 & -0.001483000 & 6 & -0.774911000 & 3.110269000 & -0.000140000 & 6 & -3.980194000 & -0.447860000 & 0.356834000 \\
\hline 6 & -3.667673000 & 1.767127000 & -0.070387000 & 6 & -0.746222000 & 2.401048000 & -1.197616000 & 6 & -3.722010000 & 0.390938000 & -0.721719000 \\
\hline 6 & -1.327974000 & 2.315691000 & 0.062803000 & 6 & -0.746216000 & 2.401165000 & 1.197405000 & 6 & -2.931383000 & -0.929054000 & 1.133210000 \\
\hline 1 & -4.703809000 & 2.073252000 & -0.120543000 & 1 & -0.778041000 & 2.926833000 & -2.143596000 & 1 & -4.534002000 & 0.763707000 & -1.329853000 \\
\hline 1 & -0.537509000 & 3.052236000 & 0.116627000 & 1 & -0.778031000 & 2.927042000 & 2.143334000 & 1 & -3.129387000 & -1.586128000 & 1.968256000 \\
\hline 6 & -3.356843000 & 0.410648000 & -0.075680000 & 6 & -0.688573000 & 1.011358000 & -1.213918000 & 6 & -2.415194000 & 0.754150000 & -1.025817000 \\
\hline 6 & -0.996887000 & 0.963091000 & 0.059033000 & 6 & -0.688567000 & 1.011477000 & 1.213842000 & 6 & -1.620270000 & -0.574498000 & 0.837721000 \\
\hline 1 & -4.138896000 & -0.332238000 & -0.129081000 & 1 & -0.685104000 & 0.475143000 & -2.151519000 & 1 & -2.208621000 & 1.406751000 & -1.863646000 \\
\hline 1 & 0.037955000 & 0.649852000 & 0.110565000 & 1 & -0.685091000 & 0.475352000 & 2.151495000 & 1 & -0.798836000 & -0.956063000 & 1.427320000 \\
\hline 6 & -2.023340000 & 0.031745000 & -0.010857000 & 6 & -0.653348000 & 0.325893000 & -0.000005000 & 6 & -1.382484000 & 0.265147000 & -0.240229000 \\
\hline 35 & -1.587251000 & -1.840785000 & -0.017742000 & 35 & -1.933912000 & -1.504168000 & 0.000087000 & 35 & 2.001688000 & -1.871195000 & -0.116710000 \\
\hline 1 & -2.904496000 & 3.772660000 & 0.001943000 & 1 & -0.824316000 & 4.189421000 & -0.000193000 & 1 & -4.997650000 & -0.729355000 & 0.589905000 \\
\hline 6 & 3.818079000 & 0.612505000 & 0.005976000 & 6 & 2.207035000 & -0.150276000 & 0.000024000 & 6 & 0.510089000 & 1.829629000 & 0.269749000 \\
\hline 1 & 3.754446000 & 1.093173000 & -0.973842000 & 1 & 2.199900000 & 0.495670000 & -0.879103000 & 1 & -0.222948000 & 2.627089000 & 0.168179000 \\
\hline 1 & 3.918613000 & 1.417690000 & 0.738412000 & 1 & 2.199954000 & 0.495641000 & 0.879172000 & 1 & 0.520515000 & 1.496401000 & 1.305144000 \\
\hline 6 & 5.063782000 & -0.275829000 & 0.054291000 & 6 & 3.456835000 & -1.031294000 & -0.000030000 & 6 & 1.889031000 & 2.268146000 & -0.195721000 \\
\hline 1 & 4.950950000 & -1.081849000 & -0.677064000 & 1 & 3.435983000 & -1.683163000 & -0.876966000 & 1 & 1.839755000 & 2.567640000 & -1.245549000 \\
\hline 1 & 5.119255000 & -0.754187000 & 1.036819000 & 1 & 3.436031000 & -1.683208000 & 0.876873000 & 1 & 2.577331000 & 1.423817000 & -0.133958000 \\
\hline 6 & 6.354693000 & 0.494916000 & -0.219446000 & 6 & 4.741014000 & -0.202936000 & -0.000045000 & 6 & 2.409813000 & 3.431622000 & 0.647757000 \\
\hline 1 & 6.335698000 & 0.956951000 & -1.208804000 & 1 & 4.797184000 & 0.437551000 & -0.882058000 & 1 & 1.746576000 & 4.295553000 & 0.584223000 \\
\hline 1 & 6.501396000 & 1.290509000 & 0.514154000 & 1 & 4.797237000 & 0.437498000 & 0.882003000 & 1 & 2.496548000 & 3.149537000 & 1.697984000 \\
\hline 1 & 7.225558000 & -0.160246000 & -0.176390000 & 1 & 5.619666000 & -0.848085000 & -0.000091000 & 1 & 3.395491000 & 3.740456000 & 0.301860000 \\
\hline 7 & 2.540418000 & -0.066446000 & 0.257102000 & 7 & 0.947579000 & -0.898671000 & 0.000050000 & 7 & -0.000576000 & 0.660660000 & -0.545105000 \\
\hline 1 & 2.556132000 & -0.503842000 & 1.172391000 & 1 & 0.838110000 & -1.484635000 & 0.820968000 & 1 & 0.650015000 & -0.161971000 & -0.405742000 \\
\hline 1 & 2.414833000 & -0.820958000 & -0.409415000 & 1 & 0.838115000 & -1.484696000 & -0.820826000 & 1 & 0.065781000 & 0.900458000 & -1.534234000 \\
\hline \multicolumn{12}{|c|}{ Water $(\varepsilon=78.36)$} \\
\hline 6 & 2.678254000 & 2.714868000 & -0.000009000 & 6 & $\begin{array}{l}-0.773927000 \\
\end{array}$ & 3.110303000 & -0.000140000 & 6 & -3.987073000 & -0.463972000 & 0.356397000 \\
\hline 6 & 1.344817000 & 2.319854000 & 0.000315000 & 6 & -0.745532000 & 2.401069000 & -1.197625000 & 6 & -3.733083000 & 0.388146000 & -0.712715000 \\
\hline 6 & 3.684398000 & 1.754759000 & -0.000320000 & 6 & -0.745528000 & 2.401186000 & 1.197414000 & 6 & -2.935343000 & -0.954448000 & 1.122918000 \\
\hline 1 & 0.557851000 & 3.062074000 & 0.000554000 & 1 & -0.777066000 & 2.926867000 & -2.143607000 & 1 & -4.547428000 & 0.768125000 & -1.313183000 \\
\hline 1 & 4.723870000 & 2.053609000 & -0.000577000 & 1 & -0.777057000 & 2.927075000 & 2.143346000 & 1 & -3.130121000 & -1.621744000 & 1.950576000 \\
\hline 6 & 1.004916000 & 0.969431000 & 0.000339000 & 6 & -0.688497000 & 1.011347000 & -1.213926000 & 6 & -2.427531000 & 0.755437000 & -1.017248000 \\
\hline 6 & 3.364789000 & 0.400318000 & -0.000308000 & 6 & -0.688492000 & 1.011465000 & 1.213850000 & 6 & -1.625424000 & -0.595845000 & 0.826927000 \\
\hline 1 & -0.033311000 & 0.663270000 & 0.000577000 & 1 & -0.685122000 & 0.475137000 & -2.151530000 & 1 & -2.224241000 & 1.418391000 & -1.847675000 \\
\hline 1 & 4.143334000 & -0.348154000 & -0.000552000 & 1 & -0.685110000 & 0.475346000 & 2.151506000 & 1 & -0.801624000 & -0.983930000 & 1.408985000 \\
\hline 6 & 2.027084000 & 0.030754000 & 0.000028000 & 6 & -0.653504000 & 0.325887000 & -0.000005000 & 6 & -1.391906000 & 0.257026000 & -0.241495000 \\
\hline 35 & 1.579099000 & -1.838998000 & 0.000047000 & 35 & -1.934373000 & -1.503720000 & 0.000087000 & 35 & 2.023816000 & -1.853669000 & -0.117659000 \\
\hline 1 & 2.932565000 & 3.765854000 & -0.000024000 & 1 & -0.822835000 & 4.189475000 & -0.000192000 & 1 & -5.003604000 & -0.748564000 & 0.589725000 \\
\hline 6 & -3.827598000 & 0.618258000 & 0.000023000 & 6 & 2.206986000 & -0.150772000 & 0.000024000 & 6 & 0.498060000 & 1.823743000 & 0.271730000 \\
\hline 1 & -3.854333000 & 1.272735000 & 0.875241000 & 1 & 2.199880000 & 0.495147000 & -0.879124000 & 1 & -0.237256000 & 2.619592000 & 0.173927000 \\
\hline 1 & -3.854256000 & 1.272926000 & -0.875055000 & 1 & 2.199934000 & 0.495119000 & 0.879192000 & 1 & 0.510830000 & 1.486870000 & 1.305932000 \\
\hline 6 & -5.067887000 & -0.278782000 & -0.000127000 & 6 & 3.456803000 & -1.031774000 & -0.000030000 & 6 & 1.875060000 & 2.267808000 & -0.194250000 \\
\hline 1 & -5.031296000 & -0.932854000 & 0.876315000 & 1 & 3.435956000 & -1.683629000 & -0.876971000 & 1 & 1.823148000 & 2.571604000 & -1.242708000 \\
\hline 1 & -5.031217000 & -0.932673000 & -0.876701000 & 1 & 3.436004000 & -1.683673000 & 0.876879000 & 1 & 2.565404000 & 1.424911000 & -0.137132000 \\
\hline 6 & -6.374750000 & 0.513471000 & -0.000104000 & 6 & 4.740956000 & -0.203371000 & -0.000045000 & 6 & 2.394195000 & 3.429033000 & 0.653349000 \\
\hline 1 & -6.447116000 & 1.154114000 & 0.881373000 & 1 & 4.797099000 & 0.437115000 & -0.882063000 & 1 & 1.728710000 & 4.291562000 & 0.594632000 \\
\hline 1 & -6.447039000 & 1.154289000 & -0.881460000 & 1 & 4.797153000 & 0.437063000 & 0.882007000 & 1 & 2.483337000 & 3.142660000 & 1.702211000 \\
\hline 1 & -7.241077000 & -0.149080000 & -0.000208000 & 1 & 5.619629000 & -0.848495000 & -0.000091000 & 1 & 3.378533000 & 3.741862000 & 0.307205000 \\
\hline 7 & -2.535327000 & -0.079232000 & 0.000002000 & 7 & 0.947536000 & -0.899157000 & 0.000050000 & 7 & -0.010877000 & 0.656101000 & -0.546334000 \\
\hline 1 & -2.471596000 & -0.686950000 & -0.809853000 & 1 & 0.838123000 & -1.485113000 & 0.820980000 & 1 & 0.640307000 & -0.165608000 & -0.408844000 \\
\hline 1 & -2.471700000 & -0.687186000 & 0.809689000 & 1 & 0.838128000 & -1.485175000 & -0.820837000 & 1 & 0.054844000 & 0.898393000 & -1.534921000 \\
\hline
\end{tabular}




\section{Complete Gaussian Reference}

Gaussian 09, Revision D.01, M. J. Frisch, G. W. Trucks, H. B. Schlegel, G. E. Scuseria, M. A. Robb, J. R. Cheeseman, G. Scalmani, V. Barone, G. A. Petersson, H. Nakatsuji, X. Li, M. Caricato, A. Marenich, J. Bloino, B. G. Janesko, R. Gomperts, B. Mennucci, H. P. Hratchian, J. V. Ortiz, A. F. Izmaylov, J. L. Sonnenberg, D. Williams-Young, F. Ding, F. Lipparini, F. Egidi, J. Goings, B. Peng, A. Petrone, T. Henderson, D. Ranasinghe, V. G. Zakrzewski, J. Gao, N. Rega, G. Zheng, W. Liang, M. Hada, M. Ehara, K. Toyota, R. Fukuda, J. Hasegawa, M. Ishida, T. Nakajima, Y. Honda, O. Kitao, H. Nakai, T. Vreven, K. Throssell, J. A. Montgomery, Jr., J. E. Peralta, F. Ogliaro, M. Bearpark, J. J. Heyd, E. Brothers, K. N. Kudin, V. N. Staroverov, T. Keith, R. Kobayashi, J. Normand, K. Raghavachari, A. Rendell, J. C. Burant, S. S. Iyengar, J. Tomasi, M. Cossi, J. M. Millam, M. Klene, C. Adamo, R. Cammi, J. W. Ochterski, R. L. Martin, K. Morokuma, O. Farkas, J. B. Foresman, and D. J. Fox, Gaussian, Inc., Wallingford CT, 2016. 